\title{
USO DE MÉTODOS BAYESIANOS EM TESTES ACELERADOS ASSUMINDO UMA DISTRIBUIÇÃO GAUSSIANA INVERSA
}

\author{
$\cdots$ \\ Of
}

Orientador : Prof. Dr. Jorge Alberto Achcar

\begin{abstract}
Dissertação apresentada ao Instituto de Ciências Matemáticas de São Carlos - USP, como parte dos requisitos para obtensão do título de Mestre em "Ciências de Computação e Matemática Computacional".
\end{abstract}

USP - SÃO CARLOS

1992 
"Em tôdas estas cousas, porêm, somos mais que vencedores, por meio daquele que nos amou." $R m 8: 97$ 
En memória a mi mamá

Fortunata,

con eterna gratitud $y$

amor.

Para mi papá Agustín

$y$ hermanos

David, Agustin, Eddy y Fernando, por todo su amor. 


\section{AGRADECIMENTOS:}

- Ao Prof. Jorge A. Achcar, por sua orientasão no decorrer do presente trabalho e incentivo à pesquisa.

- Aos Prof. Josemar Rodrigues, Maria Creusa Salles e Emanoel Barboza pelas valiosas sugestōes no exame de qualificação.

- Ao Prof. Belmer Garcia Negrillo por sua participação na banca examinadora desta dissertação.

- Aos colegas e Funcionários do ICMSC - USP.

- A Rosângela, Silvana, Lucy e Marcelo por sua amizade e convivência agradável.

- A Nilda por seu apoio e amizade.

- A CAPES pelo apoio financiero.

- Em especial, ao Mário pela amizade, pelos conselhos, compreensão, paciência e discussão de alguns pontos relativos ao trabalho. 


\section{RESUMO}

Nesta dissertação de mestrado, exploramos o uso do método de Laplace para aproximações de integrais numa análise Bayesiana da distribuição Gaussiana Inversa, considerando diferentes densidades a priori para os parâmetros do modelo. Além disso, consideramos testes acelerados, planejamentos de experimentos e esquemas de controle de qualidade a partir de densidades preditivas para a distribuição Gaussiana Inversa. Esses resultados podem ser de grande interesse prático, como é ilustrado em alguns exemplos de aplicação. 


\section{ABSTRACT}

In this dissertation we explore the use of Laplace's method for approximation of integrals in a Bayesian analysis of the Inverse Gaussian distribution, considering different prior densities for the parameters of the model. We also consider, accelerated life tests, design of experiments and quality control criteria using predictive densities for the Inverse Gaussian distribution. These results could be of great practical interest, as it is illustrated in some applied examples. 


\section{ÍNDICE}

CAPÍTULO 1 - INTRODUÇÃO E REVISÃo BIBLIOGRÁFICA

1.1 - INTRODUÇÃO

1.2 - DISTRIBUiÇÃo GaUSSIANA INVERSA NO CONTEXTO DE TESTES ACELERADOS

1.3 - REVISÃO BIBLIOGRÁFICA

CAPITULO 2 - UMA ANÁLISE DO MODELO GAUSSIANO INVERSO SEM VARIÁVEL ESTRESSE

2.1 - PARAMETRIZAÇÃO $(\theta, \lambda)$

2.1.1 - UMA ANÁLISE CLÁSSICA 6

2.1.2 - UMA ANÁLISE BAYESIANA 6

2.1.2.1 DENSIDADE A PRIORI CONJUGADA NATURAL 14

2.1.2.2 DENSIDADE A PRIORI DE JEFFREYS

2.2 - PARAMETRIZAÇÃO $(\mu, \lambda)$

2.2.1 - UMA ANÁLISE CLÁSSICA 21

2.2.2. UMA ANÁLISE BAYESIANA

2.2.2.1 - DENSIDADE A PRIORI CONJUGADA NATURAL 23

2.2.2.2 - DENSIDADE A PRIORI DE JEFFREYS 24

2.2.2.3 - DENSIDADE A PRIORI IMPRÓPRIA

CAPÍTULO 3 - UMA ANÁlISE CLÁSSICA PARA O MODELO DE REGRESSÃO LINEAR INVERSO

3.1 - O MODELO DE REGRESSÃO LINEAR INVERSO

3.2 - O EXPERIMENTO EM TESTES ACELERAdos COM A RELAÇÃo

PARÂMETRO - ESTRESSE

3.3 - ESTIMADORES DE MÁXIMA VEROSSIMILHANÇA

3.4 - INFERÊNCIAS SOBRE OS PARÁMETROS

3.5 - INTERVALOS DE CONFIANÇA PARA OS PARÁMETROS 
Capítulo 4 - UMa anÁlise bayesiana PARA O MOdElo de REGRESSÃo LINEAR INVERSO

4.1 - UMA ANÁLISE BAYESIANA ASSUMINDO $\alpha, \beta$ e $\lambda$ DESCONHECIDOS 42

4.1.1 - FUNÇĀO DE VEROSSIMILHANÇA PARA $\alpha, \beta$ e $\lambda$. 42

4.1.2 - DENSIDADE A PRIORI PARA $\alpha, \beta$ e $\lambda \quad 42$

4.1.3 - DENSIDADE A POSTERIORI CONJUNTA PARA $\alpha, \beta$ e $\lambda$

4.1.4 - DENSIDADE A POSTERIORI MARGINAL PARA $\alpha, \beta$ e $\lambda$

4.1.5 - DENSIDADE A POSTERIORI MARGINAL PARA O TEMPO MÉDIO DE FALHA $\theta_{1}$ SOB O NÍVEL USUAL DE ESTRESSE $X_{1}$. 46

4.2 - UMA ANÁLISE BAYESIANA ASSUMINDO $\lambda$ CONHECIDO 48

4.2.1 - DENSIDADE A POSTERIORI CONJUNTA PARA $\alpha$ e $\beta \quad 48$

4.2.2 - DENSIDADE A POSTERIORI MARGINAI PARA $\alpha \in \beta$

4.2.3 - DENSIDADE A POSTERIORI MARGINAL PARA O TEMPO MÉdIO DE FALHA $\theta_{1}$ SOB O NÍVEL USUAL DE ESTRESSE $X_{1}$ COM $\lambda$ CONHECIDO $\quad 50$

4.3 - INTERVALOS DE CREDIBILIDADE PARA OS PARÂMETROS

$\begin{array}{ll}\text { CAPÍTULO } 5 \text { - DENSIDADES PREDITIVAS } & 57\end{array}$

5.1 - DENSIDADE PREDITIVA PARA UMA OBSERVAÇÃO FUTURA

5.2 - USO DA DENSIDADE PREDITIVA EM CONTROLE DE QUALIDADE 60

CAPÍtulo 6 - PLANEJAMENTOS DE EXPERIMENTOS EM TESTES ACELERADOS COM O MODELO DE REGRESSÃO LINEAR INVERSO

6.1 - UMA ANÁLISE CLÁSSICA

6.1.1 - FORMULAÇÃO DO MODELO

6.1.2 - ESTIMADORES DE MÁXIMA VEROSSIMILHANÇA 64

6.1.3 - ESTIMADORES NÃO - VICIADOS VIA MINIMOS QUADRADOS 70

6.2 - UMA ANÁLISE BAYESIANA

6.2.1 - DENSIDADE A PRIORI NÃO-INFORMATIVA PARA $\Phi$ e $\sigma$.

6.2.2 - DENSIDADE A POSTERIORI CONJUNTA PARA $\Phi$ e $\sigma$

6.2.3 - DENSIDADE A POSTERIORI MARGINAL PARA $\Phi$

6.2.4 - DENSIDADE A POSTERIORI MARGINAL PARA $\sigma$

6.2.5 - INTERVALOS DE CREDIBILIDADE PARA OS PARÁMETROS 
CAPÍTULO 7 - APLICAÇÕES NUMÉRICAS

7.1 - ANÁlISE DO MODELO GAUSSIANO INVERSO SEM VARIÁVEL ESTRESSE - ANÁLISE CLÁSSICA E BAYESIANA

7.1.1 - PRIMEIRO EXEMPLO

7.1.2 - SEGUNDO EXEMPLO

7.2 - ANÁLISE DO MODELO DE REGRESSÃO LINEAR INVERSO-ANÁLISE CLÁSSICA E BAYESIANA

7.2.1 - TERCEIRO EXEMPLO

7.3 - PLANEJAMENTOS DE EXPERIMENTOS EM TESTES ACELERADOS COM O MODELO DE REGRESSÃO LINEAR INVERSO - ANÁLISE CLÁSSICA E BAYESIANA 105

7.3.1 - QUARTO EXEMPLO

CAPÍTULO 8 - CONCLUSÕES E CONSIDERAÇÕES FUTURAS

REFERÉNCIAS BIBLIOGRÁFICAS

APÊNDICES

A.1 - O MÉTODO DE LAPLACE

A.2 - O MÉTODO DE SIMPSON

A.3 - INFERÊNCIAS PARA OS PARÂMETROS A PARTIR DA TEORIA ASSINTÓTICA 


\section{CAPÍTULO 1}

\section{INTRODUCÃO E REVISÃO BIBLIOGRÁFICA}

\subsection{INTRODUÇÃO.}

Em certos experimentos de testes de vida, é comum na prática submeter os itens em teste a condições altas de estresse. Tais procedimentos são chamados testes de vida acelerados. Estes testes são aplicados em produtos e materiais para se obter informaçōes rápidas sobre as distribuições dos tempos de vida e são realizados expondo as unidades testadas em condições que são mais rigorosas que as normais. Estes resultados em unidades com tempos de vida pequenos poderiam ser observadas sob condiçōes normais. Os resultados obtidos nas condições aceleradas são então extrapoladas para as condiçôes normais para obter uma estimativa da distribuição do tempo de vida sob as condiçōes normais.

Os procedimentos usuais em testes acelerados são tipicamente produzidos para testar unidades em altos niveis de temperatura, voltagem, pressão, vibração, razão cíclica, péso, etc. ; ou algumas combinações delas que são encontradas sob condições normais. A escolha destas variáveis aceleradas, como são usualmente chamadas, para um produto ou material é usualmente estabelecido pela prática em engenharia.

Uma análise paramétrica de testes de vida acelerados depende muito do modelo escolhido para a distribuição do tempo de vida e da relação dos parâmetros dessa distribuição com a variável estresse. Além das considerações para o ajuste empírico, uma distribuição do tempo de vida deriva de postulados razoáveis do processo de falha, fortalecendo assim seu uso estatistico.

Para a relação parâmetro-estresse, existem alguns modelos empíricos comumente usados em engenharia tais como o modelo Arrhenius, Eyring e de Potência Inversa. Alguns procedimentos na inferência com essas formulaçōes são discutidas em Nelson (1990), Mann, Schaffer e Singpurwalla (1974). 


$$
1.1 \text { - introdução - pág. } 2
$$

Neste primeiro capítulo são apresentados a distribuiçāo Gaussiana Inversa e o Modelo de Regressão Linear Inverso, e posteriormente uma breve revisão bibliográfica do modelo. No capítulo 2, apresentamos uma Análise Clássica e Bayesiana do modelo sem variável estresse, com suas densidades a posteriori marginais $e$ os intervalos de confiança e de credibilidade aproximados, utilizando as parametrizaçōes $(\theta, \lambda)$ e $(\mu=1 / \theta, \lambda)$. Consideramos a densidade a priori conjugada natural, a priori de Jeffreys e uma densidade a priori imprópria para este modelo. No capitulo 3, apresentamos uma Análise Clássics do Modelo de Regressão Linear Inverso e nos referimos à base física da distribuição Gaussiana Inversa para formular uma relação estocástica razoável entre o tempo médio de falha e a variável estresse. Além disso encontramos os estimadores de máxima verossimilhança e os intervalos de confiança correspondentes para os parâmetros. No capítulo 4 fazemos uma Análise Bayesiana para o modelo de Regressão Linear Inverso, inicialmente assumindo que os parāmetros sejam desconhecidos e posteriormente assumindo um deles conhecido. Encontramos a densidade a posteriori marginal para o tempo médio de falha $\theta_{1}$ sob o nivel usual de estresse $x_{1}$ e calculamos os seus intervalos de credibilidade aproximados. Encontramos ainda a densidade preditiva para uma observação futura (ver capitulo 5 ), onde apresentamos também alguns critérios para serem usados em controle de qualidade. No capitulo 6, consideramos uma análise clássica e Bayesiana num planejamento de experimentos em testes acelerados com o modelo de Regressão Linear Inverso. Neste capítulo são mostrados os efeitos fatoriais no contexto do processo de Wiener. Também são dados os estimadores de máxima verossimilhança e o6 respectivos intervalos de confiança aproximados para os parâmetros e encontramos os estimadores de mínimos quadrados usando os inversos das médias das celas amostrais. Para a análise Bayesiana é considerada uma densidade a priori não-informativa para se obter as densidades a posteriori para os parâmetros de interesse. No capítulo 7 são apresentadas algumas aplicações numéricas. Um primeiro conjunto de dados introduzido por Achcar, Bolfarine e Rodrigues (1987), e um segundo introduzido por Lieblin e Żelen (1956), são utilizados para fazer uma Análise Clássica e Bayesiana do modelo sem variável estresse. Num outro exemplo, com um conjunto de dados introduzido por Nelson (1971), é feita uma análise clássica e Bayesiana do modelo de Regressão Linear Inverso e é mostrado o uso da densidade preditiva para uma observação futura em controle de qualidade. No último exemplo, com dados introduzidos por Shuster e Miura (1972), é feita uma análise clássica e Bayesiana em planejamento de experimentos. O Método de Laplace, utilizado para aproximar integrais é usado para aproximar densidades a posteriori marginais (ver apéndice A1). O Método de Simpson, também utilizado com densidades preditivas é apresentado no apêndice A2 e alguns resultados sobre as inferências para os parâmetros a partir da teoria assintótica sâo dadas no apêndice A3. No apêndice A4 são apresentados alguns programas computacionais (utilizando o pacote estatístico SOC - Software Científico desenvolvido pelo NTIA/EMBRAPA) utilizados para a aplicasāo numérica. 
1.2 - distribuição Gaussiana Inversa no contexto de testes acelerados - pág. 9

\subsection{DISTRIBUIÇĀO GAUSSIANA INVERSA NO CONTEXTO DE TESTES ACELERADOS}

Assumimos uma distribuição de sobrevivência versátil mas não muito conhecida, chamada Distribuição Gaussiana Inversa IG $(\theta, \lambda)$, cuja função densidade de probabilidade (f.d.p.) é dada por,

$$
f(y ; \theta, \lambda)=\left(2 \pi \lambda^{-1} y^{3}\right)^{-1 / 2} \exp \left\{-\frac{1}{2} \lambda y^{-1}\left(y \theta^{-1}-1\right)^{2}\right\}
$$

onde $y>0, \theta>0$ e $\lambda>0$.

Esta distribuição apresenta trés caracteristicas desejáveis: uma ampla variedade de forma da curva de densidade de probabilidade, tratabilidade analitica dos resultados de inferència, e uma motivação dada a partir do processo estocástico de falha. As propriedades básicas desta distribuição são dadas em Tweedie (1957) e um levantamento bibliográfico extensivo é dado por Folks e Chhikara (1978).

No contexto de testes acelerados, usualmente nos referimos à base física da distribuição Gaussiana Inversa para formular uma relação estocástica razoável do tempo de falha para a variável estresse. Um modelo resultante tem a forma linear inversa dada por,

$$
\theta^{-1}=\alpha+\beta \mathrm{X}
$$

onde $\alpha \geq 0, \beta \geq 0, \alpha+\beta>0$ e X $>0$ (ver por exemplo, Bhattacharyya e Fries, 1981).

Uma formulação diferente foi dada por Davis (1977) que considerou o tradicional modelo de regressão linear $\theta=\alpha+\beta \mathrm{X}$ assumindo $\lambda$ constante. 


\subsection{REVISÃO BIBLIOGRÁFICA.}

A gênese da distribuição Gaussiana Inversa data desde 1915 quando Schrödinger deduziu a distribuiçâo de probabilidade do tempo de primeira passagem num movimento Browniano. Depois disso, encontramos um artigo de Tweedie (1945), que formulou a relação inversa da função geratriz cumulante da distribuiçāo com aquela da distribuiçāo normal, dá propondo o nome de distribuição Gaussiana Inversa. Wald (1947), derivou a distribuição Gaussiana Inversa numa forma limitante para a distribuição do tamanho amostral numa análise sequencial, por isso também é conhecida como a distribuição de Wald principalmente na literatura russa. Outros nomes para a distribuição são dados por distribuição do tempo de primeira passagem Gaussiano (Moran, 1968) e a distribuição do tempo de primeira passagem num movimento Browniano com direção positiva (Wasan, 1967).

Tweedie (1957a, 1957b) estudou suas caracteristicas básicas estabelecendo propriedades estatísticas importantes e descreveu certas analogias com as propriedades da distribuição normal. A caracterização da distribuição Gaussiana Inversa por Khatri (1962) mostra a caracterização usual da distribuição amostral pela independência da media amostral e variância. Shuster (1968) expressa a função distribuição acumulada da distribuição Gaussiana Inversa em termos da função distribuição acumulada da distribuição normal. Wasan e Roy (1969) apresentam algumas características e propriedades analiticas desta classe de distribuição principalmente para as formas limitantes além de uma tabela dos valores das porcentagens pontuais da distribuição.

Mais recentemente, Chhikara (1975) e Chhikara e Folks (1974, 1975, 1976) provaram algumas propriedades adicionais da distribuição Gaussiana Inversa, e encontraram estimadores nãoviciados de minima variância para a função distribuição acumulada e algumas distribuiçōes amostrais que também foram obtidas por Tweedie (1957a).

Chhikara e Folks (1977), sugerem o uso da distribuição Gaussiana Inversa como um modelo de tempo de vida, discutem os diversos fatores diferentes de confiabilidade desta distribuição e ainda apresentam as vantagens em relação ao uso da distribuição log-normal. A partir dessa sugestão, Padgett (1979) apresenta limites de confiança para a função de confiabilidade $(R(t)=P(T>t))$ considerando dois casos: no primeiro, o parâmetro de forma é conhecido e o parâmetro de escala desconhecido, e no segundo caso, o parâmetro de forma é desconhecido e o de escala é conhecido. Posteriormente, Padgett (1981) considera a estimação da função de confiabilidade sob o ponto de vista Bayesiano para a distribuição Gaussiana Inversa. No contexto de testes de sobrevivência acelerados, Bhattacharyya e Fries (1981) relacionam a base física desta distribuição para formular uma relação estocástica razoável para o tempo de falha com a intensidade da variável estresse.

Banerjee (1977) e Al-Hussaini e Abb-El-Hakim (1981) descrevem a distribuição Gaussiana Inversa no caso bivariado, assim como suas relaçōes com a distribuição normal e qui-quadrado, e 
também propōem uma generalização para o caso multivariado.

Alguns resultados Bayesianos são obtidos por Banerjee e Bhattacharyya (1979) que utilizam prioris de referência não-informativas e também a priori conjugada, utilizando uma parametrização adequada para encontrar densidades a posteriori para os parâmetros de interesse. Eles observam uma grande similaridade com os resultados correspondentes da distribuição normal. Achcar, Bolfarine e Rodrigues (1987) também apresentam uma análise Bayesiana do modelo Gaussiano Inverso considerando uma priori não-informativa de Jeffreys para os parámetros de interesse.

Cheng e Amin (1981) sugerem uma distribuição alternativa, a distribuiçāo Gaussiana Inversa com três parâmetros (origem desconhecida). Consideram a estimação de máxima verossimilhança e mostram que não há dificuldade em achar estas estimativas, o que não acontece com outras distribuições conhecidas tais como, lognormal, gama e Weibull.

Bhattacharyya e Fries (1982) apresentam e discutem a relação existente entre a distribuição Gaussiana Inversa e a distribuição de Birnbaum-Saunders. A primeira é obtida pela derivação exata enquanto que a segunda envolve certas aproximaçōes. Eles mostram que ambas se ajustam muito bem aos dados contendo tempos de falha; no entanto mostram que a distribuição Gaussiana Inversa tem algumas vantagens com relação à análise e a tratabilidade das distribuições amostrais.

Bhattacharyya e Fries (1983) consideram uma análise de experimentos fatoriais utilizando a distribuição Gaussiana Inversa e um modelo Linear Inverso para os efeitos dos fatores motivado sob o contexto de um processo de Wiener.

Cheng (1984) descreve um método para a geração de uma amostra aleatória da variável Gaussiana Inversa a partir de uma média amostral e uma medida de dispersão. Este método pode ainda ser estendido para produzir amostras de outras distribuições.

Doksum e Hoyland (1992) consideram modelos para a variável estresse de testes de vida acelerados para experimentos baseados no processo de Wiener e a distribuição Gaussiana Inversa, onde cada unidade do experimento é sujeita a certos modelos específicos de estresse e intervalo de tempo, até a primeira falha. Consideram também o caso em que unidades diferentes são sujeitas a diferentes modelos de estresse por etapas onde o tempo do teste é reduzido antes que todas as unidades tenham falhado, ou seja, os dados são censurados.

Outros trabalhos recentes relacionados ao estudo de Testes Acelerados podem ser encontrados em Louzada Neto (1991) que desenvolvem o Teste de Sobrevivência Acelerados considerando o uso da análise Bayesiana para o modelo de Eyring com uma variável estresse e Dias (1992) que analizam o Modelo de Lei de Potência Inversa assumindo uma distribuição exponencial para os tempos de sobrevivência de componentes com censuras de tipo II. 


\section{CAPITULO 2}

\section{UMA ANÁLISE DO MODELO GAUSSIANO INVERSO SEM VARIÁVEL ESTRESSE}

\subsection{PARAMETRIZAÇÃo $(\theta, \lambda)$.}

Em geral pode ser notado que uma boa parametrização $(\theta, \lambda)$ ajuda muito no uso da metodologia estatística de estimação paramétrica e testes de hipótese. Então é desejável sempre que possivel fixar esta parametrização.

\subsubsection{UMA ANÁLISE CLÁSSICA.}

Para a análise clássica será apresentada a função densidade de probabilidade (f.d.p.) da distribuição Gaussiana Inversa e suas principais propriedades. Para uma variável aleatória Y que segue essa distribuição com parämetros $\theta$ e $\lambda$ temos,

$$
f(y ; \theta, \lambda)=\left(2 \pi \lambda^{-1} y^{3}\right)^{-1 / 2} \exp \left\{-\frac{1}{2} \lambda y^{-1}\left(y \theta^{-1}-1\right)^{2}\right\}
$$

onde $y>0, \theta>0$ e $\lambda>0$. Essa densidade será denotada por IG $(\theta, \lambda)$ e escrevemos $\mathrm{Y} \sim$ IG $(\theta, \lambda)$. A f.d.p (2.1) é unimodal e assimetricamente positiva, o parämetro $\theta$ é a média da distribuição e $\lambda$ é um parâmetro de forma.

A função característica da distribuição Gaussiana Inversa é dada por,

$$
\phi(t)=\exp \left\{\left(\frac{\lambda}{\theta}\right)\left[1-\left(1-\frac{2 \mathrm{i} t \theta^{2}}{\lambda}\right)^{1 / 2}\right]\right\}
$$




\section{1 - parametrização $(\theta, \lambda)$ - pág. 7}

Consequentemente, segue-se que todos os momentos existem e, em particular temos que,

$$
E(Y)=\theta \quad \text { e } \quad V(Y)=\frac{\theta^{3}}{\lambda}
$$

Tweedie (1957a) apresenta também outras formas equivalentes desta distribuição as quais podem ser obtidas substituindo o conjunto de parâmetros $(\theta, \lambda)$ por $(\tau, \lambda),(\theta, \phi)$ ou $(\phi, \lambda)$ usando a seguinte relação, $\frac{1}{2} \tau^{2}=\theta=\frac{\lambda}{\phi}$, onde as funções densidades para valores positivos da variável podem ser escritas como,

$$
\begin{aligned}
& f(y ; \tau, \lambda)=\left(2 \pi \lambda^{-1} y^{3}\right)^{-1 / 2} \exp \left\{-\tau \lambda y+\lambda(2 \tau)^{1 / 2}-\lambda / 2 y\right\} \\
& f(y ; \theta, \phi)=\left(2 \pi[\theta \phi]^{-1} y^{3}\right)^{-1 / 2} \exp \left\{-\frac{\phi y}{2 \theta}+\phi-\frac{\theta \phi}{2 y}\right\} \\
& f(y ; \phi, \lambda)=\left(2 \pi \lambda^{-1} y^{3}\right)^{-1 / 2} \exp \left\{-\frac{\phi^{2} y}{2 \lambda}+\phi-\frac{\lambda}{2 y}\right\}
\end{aligned}
$$

assim a relação entre as três funções é dado por,

$$
f(y ; \theta, \lambda)=\theta^{-1} \mathbf{f}\left(\frac{y}{\theta} ; 1, \phi\right)=\lambda^{-1} f\left(\frac{y}{\lambda} ; \phi, 1\right)
$$

Cada uma dessas formas pode ser conveniente para propósitos específicos da Teoria do Movimento Browniano. A forma da distribuição depende somente de $\phi$, portanto $\phi$ é o parâmetro de forma da distribuição. A f.d.p Gaussiana Inversa representa uma ampla classe de distribuiçōes, abrangendo distribuições altamente assimétricas até uma distribuição quando varia $\phi$ de 0 a $\infty$. As curvas da densidade são dadas nos seguintes gráficos: 


\section{GRÁFICO 2.1}

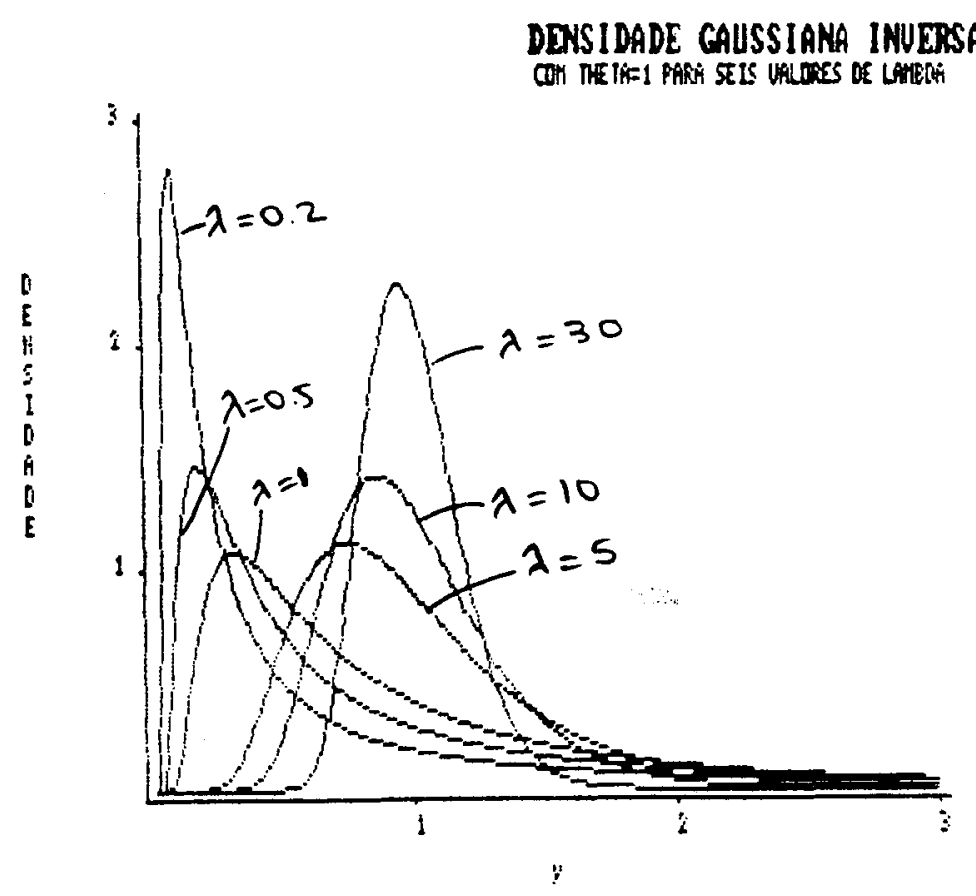

GRÁFICO 2.2

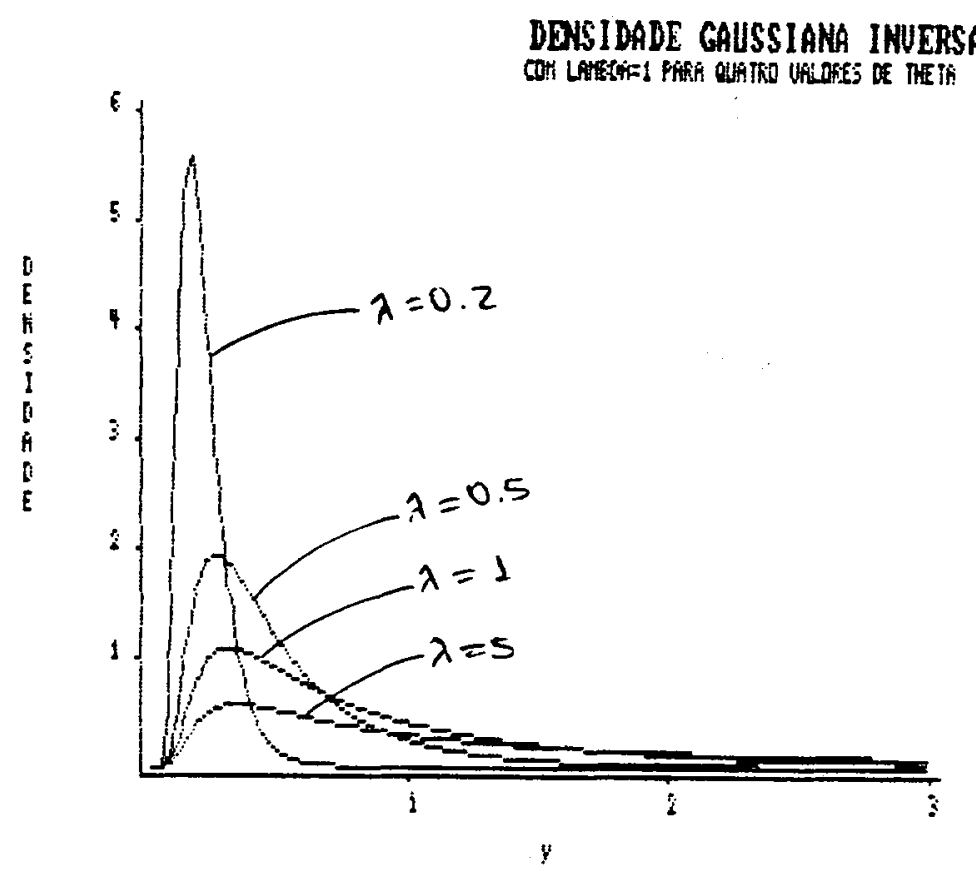

Essas curvas são obtidas especificando $\theta=1$ e variando $\lambda$ ou $\phi$ no gráfico 2.1 e especificando $\lambda=1$ e variando $\theta$ ou $\phi$ no gráfico 2.2 . 
Pode notado que a família da distribuição Gaussiana Inversa pertence à família exponencial de ordem 2. Fazendo $\varphi=\frac{\lambda}{\theta^{2}}$, a f.d.p (2.1) pode ser eacrita por,

$$
f(y ; \lambda, \varphi)=\left(\frac{\lambda}{2 \pi}\right)^{1 / 2} y^{-3 / 2} \exp \left\{(\lambda \varphi)^{1 / 2}\right\} \exp \left\{-\frac{1}{2}\left(\lambda y^{-1}+\varphi y\right)\right\}
$$

que é da forma da familia exponencial, $a(\psi) b(y) \exp \{\psi t(y)\}$, onde $\psi=(\lambda, \varphi), t(y)=(-1 / 2)\left(y^{-1}, y\right)$, $b(y)=y^{-3 / 2}$ e $a(\psi)=\left(\frac{\lambda}{2 \pi}\right)^{1 / 2} \exp \left\{(\lambda \varphi)^{1 / 2}\right\}$

Para a estimação dos parâmetros, consideramos $Y_{1}, Y_{2}, \ldots, Y_{n}$ uma amostra aleatória simples de uma distribuição Gaussiana Inversa com densidade (2.1). A função de verossimilhança para $\theta$ e $\lambda$ em (2.1) é dada por,

$$
L(\theta, \lambda)=(2 \pi)^{-n / 2} \lambda^{n / 2} \prod_{i=1}^{n} y_{i}^{-3 / 2} \exp \left\{-\left(\frac{1}{2} \lambda \theta^{-2} \sum_{i=1}^{n} y_{i}-n \lambda \theta^{-1}+\frac{1}{2} \lambda \sum_{i=1}^{n} y_{i}^{-1}\right)\right\}
$$

Definindo $\bar{Y}=n^{-1} \sum_{i=1}^{n} y_{i}$ e $R=n^{-1} \sum_{i=1}^{n} y_{i}^{-1}$, e aplicando a função log em (2.7) temos que,

$$
\mathrm{l}(\theta, \lambda)=-\frac{\mathrm{n}}{2} \log 2 \pi+\frac{\mathrm{n}}{2} \log \lambda-\frac{3}{2} \sum_{i=1}^{n} \log y_{i}-\frac{1}{2} \lambda \theta^{-2} \mathrm{n} \overline{\mathrm{Y}}+\mathrm{n} \lambda \theta^{-1}-\frac{1}{2} \lambda \mathrm{nR}
$$

As primeiras derivadas de $\mathrm{l}(\theta, \lambda)(2.8)$ com respeito a $\theta$ e $\lambda$ são,

$$
\begin{aligned}
& \frac{\partial \log \mathrm{l}(\theta, \lambda)}{\partial \theta}=\lambda \theta^{-3} \mathrm{n} \overline{\mathrm{Y}}-\mathrm{n} \lambda \theta^{-2} \\
& \frac{\partial \log \mathrm{l}(\theta, \lambda)}{\partial \lambda}=\frac{\mathrm{n}}{2} \frac{1}{\lambda}-\frac{1}{2} \theta^{-2} \mathrm{n} \overline{\mathrm{Y}}+\mathrm{n} \theta^{-1}-\frac{1}{2} \mathrm{nR}
\end{aligned}
$$

Igualando a zero as primeiras derivadas (2.9), obtemos os EMV de $\theta$ e $\lambda$ dados respectivamente por,

$$
\begin{aligned}
& \hat{\theta}=\bar{Y} \\
& \dot{\lambda}=\frac{1}{\left(R-\bar{Y}^{-1}\right)} \text { e } \hat{\lambda}^{-1}=\left(R-\bar{Y}^{-1}\right)
\end{aligned}
$$

Para verificar se um determinado modelo se ajusta bem a um conjunto de dados, várias estatisticas podem ser utilizadas. Em particular, a estatistica de Kolmogorov-Smirnov (K-S) pode ser a indicada para testar se a distribuição Gaussiana Inversa está bem ajustada. Dada uma amostra aleatória de tamanho n, a estatítica de K-S é definida por (Chhikara e Folks, 1989), 


$$
\mathrm{D}_{o b o}^{K S}=\max \left(\mathrm{D}_{o b \bullet}^{K S+}, \mathrm{D}_{o b \bullet}^{K S-}\right)
$$

onde $\mathrm{D}_{o b \boldsymbol{b}}^{K S+}=\max _{1 \leq i \leq n}[\mathrm{i} / \mathrm{n}-\mathrm{F}(\mathrm{y})]$ e $\mathrm{D}_{o b \bullet}^{K S-}=\max _{1 \leq i \leq n}[\mathrm{~F}(\mathrm{y})-(\mathrm{i}-1) / \mathrm{n}]$ Para este caso, a funsão distribuição hipotética é dada por,

$$
F(y)=\Phi\left[\sqrt{\frac{\lambda}{y}}\left(\frac{y}{\theta}-1\right)\right]+\exp \left(\frac{2 \lambda}{\theta}\right) \Phi\left[-\sqrt{\frac{\lambda}{y}}\left(1+\frac{y}{\theta}\right)\right]
$$

onde $\Phi(y)$ é a F.D.A da distribuição normal.

Para o teste de ajuste do modelo é utilizado a equação,

$$
\mathrm{D}_{c r i}^{K S}=\beta_{0}^{K S}+\beta_{1}^{K S}\left(\mathrm{n}^{-1 / 2}\right)+\beta_{2}^{K S}\left(\phi^{-1 / 2}\right)
$$

onde $\phi=\lambda / \theta$ e os valores de $\beta_{0}^{K S}, \beta_{1}^{K S}, \beta_{2}^{K S}$ são dados na tabela a seguir,

TABELA 2.1 - Coeficientes para o nivel $\gamma$ e classe $\phi$

\begin{tabular}{|l|lll|lll|}
\hline \multicolumn{3}{|c|}{$\phi \leq 1$} \\
\hline$\gamma$ & $\beta_{0}^{K S}$ & $\beta_{1}^{K S}$ & $\beta_{2}^{K S}$ & $\beta_{0}^{K S}$ & $\beta_{1}^{K S}$ & $\beta_{2}^{K S}$ \\
\hline 0.200 & -0.048 & 0.7381 & 0.0668 & 0.004 & 0.6631 & 0.0206 \\
0.150 & -0.052 & 0.7736 & 0.0727 & 0.005 & 0.6909 & 0.0218 \\
0.100 & -0.057 & 0.8173 & 0.0791 & 0.006 & 0.7254 & 0.0237 \\
0.050 & -0.064 & 0.8864 & 0.0907 & 0.008 & 0.7773 & 0.0269 \\
0.025 & -0.069 & 0.9498 & 0.0998 & 0.011 & 0.8215 & 0.0297 \\
0.010 & -0.070 & 1.0170 & 0.1086 & 0.014 & 0.8689 & 0.0355 \\
\hline
\end{tabular}

fonte: Stark e Chhikara, 1988.

Portanto, aceitamos que o ajuste é bom se $\mathrm{D}_{\text {obs }}^{K S}$ for menor que o $\mathrm{D}_{c r i}^{K S}$ ao nivel de significância especificado (Stark e Chhikara, 1988).

As inferências para $\psi=(\theta, \lambda)^{\prime}$ podem ser baseadas na aproximação normal assintótica dos estimadores de máxima verossimilhança $\bar{\psi}=(\hat{\theta}, \hat{\lambda})^{\prime}$ considerando a matriz de informação observada $\mathrm{I}_{0}$ ou a matriz de informação de Fisher I $(\psi)$ ( Mood, Graybill e Boes, 1974 ; Lawless, 1982 ). 
A matriz de informaçāo observada é dada por,

$$
I_{0}=\left\{-\frac{\partial^{2} 1(\theta, \lambda)}{\partial \psi^{2}}\right\} \mid \psi=\dot{\psi}
$$

As segundas derivadas parciais com sinal negativo da função log-verossimilhança (2.8) com respeito a $\theta$ e $\lambda$ sâo,

$$
\begin{aligned}
& -\frac{\partial^{2} \mathrm{l}(\theta, \lambda)}{\partial \theta^{2}}=3 \lambda \theta^{-4} \mathrm{n} \overline{\mathrm{Y}}-2 \mathrm{n} \lambda \theta^{-3} \\
& -\frac{\partial^{2} \mathrm{l}(\theta, \lambda)}{\partial \lambda^{2}}=\frac{\mathrm{n}}{2 \lambda^{2}} \\
& -\frac{\partial^{2} \mathrm{l}(\theta, \lambda)}{\partial \theta \partial \lambda}=n \theta^{-2}-\theta^{-3} \mathrm{n} \bar{Y}=-\frac{\partial^{2} \mathrm{l}(\theta, \lambda)}{\partial \lambda \partial \theta}
\end{aligned}
$$

Calculando (2.13) em $\hat{\theta}$ e $\hat{\lambda}$ temos,

$$
\begin{aligned}
& -\left.\frac{\partial^{2} \mathrm{l}(\theta, \lambda)}{\partial \theta^{2}}\right|_{\psi=\hat{\psi}}=\frac{\mathrm{n} \overline{\mathrm{Y}}^{-3}}{\left(\mathrm{R}-\overline{\mathrm{Y}}^{-1}\right)} \\
& -\left.\frac{\partial^{2} \mathrm{l}(\theta, \lambda)}{\partial \lambda^{2}}\right|_{\psi=\dot{\psi}}=\frac{\mathrm{n}\left(\mathrm{R}^{2}-2 \mathrm{R} \overline{\mathrm{Y}}-1+\overline{\mathrm{Y}}^{-2}\right)}{2} \\
& -\left.\frac{\partial^{2} \mathrm{l}(\theta, \lambda)}{\partial \theta \partial \lambda}\right|_{\psi=\dot{\psi}}=0=-\left.\frac{\partial^{2} \mathrm{l}(\theta, \lambda)}{\partial \lambda \partial \theta}\right|_{\psi=\dot{\psi}}
\end{aligned}
$$

Portanto, substituindo as equações de (2.14) em (2.12) temos,

$$
\mathrm{I}_{0}=\left[\begin{array}{cc}
\frac{\mathrm{n} \overline{\mathrm{Y}}^{-3}}{\left(\mathrm{R}-\overline{\mathrm{Y}}^{-1}\right)} & 0 \\
0 & \frac{\mathrm{n}\left(\mathrm{R}^{2}-2 \mathrm{R} \overline{\mathrm{Y}}^{-1}+\overline{\mathrm{Y}}^{-2}\right)}{2}
\end{array}\right]
$$

Para fazer inferências para $\psi=(\theta, \lambda)^{\prime}$, podemos usar o fato que para n grande,

$$
\hat{\psi}=(\hat{\theta}, \hat{\lambda})^{\prime} \approx N\left\{(\theta, \lambda)^{\prime} ; I_{0}^{-1}\right\}
$$


Logo, as inferências pars $\psi=(\theta, \lambda)^{\prime}$ podem ser baseadas em $(2.16)$.

De (2.15),

$$
I_{0}^{-1}=\left[\begin{array}{cc}
\frac{\left(R-\bar{Y}^{-1}\right)}{n \bar{Y}^{-3}} & 0 \\
0 & \frac{2}{n\left(R^{2}-2 R \bar{Y}^{-1}+\bar{Y}^{-2}\right)}
\end{array}\right]
$$

Portanto, podemos encontrar as variâncias aproximadas para $\hat{\theta}$ e $\hat{\lambda}$ e construir intervalos de confiança ou testes de significância para $\theta$ e $\lambda$. As variâncias aproximadas para $\hat{\theta}$ e $\hat{\lambda}$ são dadas por,

$$
\begin{aligned}
& \hat{\mathrm{V}}(\hat{\theta})=\left(\mathrm{R}-\overline{\mathrm{Y}}^{-1}\right)\left(\mathrm{n} \overline{\mathrm{Y}}^{-3}\right)^{-1} \\
& \hat{\mathrm{V}}(\hat{\lambda})=2\left\{\mathrm{n}\left(\mathrm{R}^{2}-2 \mathrm{R} \overline{\mathrm{Y}}^{-1}+\overline{\mathrm{Y}}^{-2}\right)\right\}^{-1}
\end{aligned}
$$

A matriz de informação de Fisher para $\theta$ e $\lambda$ é dada por,

$$
I(\psi)=E\left\{-\frac{\partial^{2} \mathrm{I}(\theta, \lambda)}{\partial \psi^{2}}\right\}
$$

substituindo as equaçōes de (2.13) em (2.19) temos que,

$$
I(\psi)=E\left[\begin{array}{cc}
\left(3 \lambda \theta^{-4} \mathrm{n} \overline{\mathrm{Y}}-2 \mathrm{n} \lambda \theta^{-3}\right) & \left(\mathrm{n} \theta^{-2}-\theta^{-3} \mathrm{n} \overline{\mathrm{Y}}\right) \\
\left(\mathrm{n} \theta^{-2}-\theta^{-3} \mathrm{n} \overline{\mathrm{Y}}\right) & \frac{\mathrm{n}}{2 \lambda^{2}}
\end{array}\right]
$$

onde,

$$
\begin{aligned}
& E\left\{\left(3 \lambda \theta^{-4} n \bar{Y}-2 n \lambda \theta^{-3}\right)\right\}=n \lambda \theta^{-3} \\
& E\left\{\left(n \theta^{-2}-\theta^{-3} n \bar{Y}\right)\right\}=0 \\
& E\left(\frac{n}{2 \lambda^{2}}\right)=\frac{n}{2 \lambda^{2}}
\end{aligned}
$$


Portanto, substituindo (2.21) em (2.20) temos que,

$$
\mathbf{I}(\psi)=\left[\begin{array}{cc}
\mathrm{n} \lambda \theta^{-3} & 0 \\
0 & \frac{\mathrm{n}}{2 \lambda^{2}}
\end{array}\right]
$$

Assim de (2.22),

$$
I^{-1}(\psi)=\left[\begin{array}{cc}
\frac{1}{n \lambda \theta^{-3}} & 0 \\
0 & \frac{2 \lambda^{2}}{n}
\end{array}\right]
$$

Portanto, desta matriz temos que as variâncias aproximadas para $\hat{\theta}$ e $\hat{\lambda}$ são dadas por,

$$
\begin{aligned}
& \hat{\mathrm{v}}(\hat{\theta})=\left(\mathrm{n} \lambda \theta^{-3}\right)^{-1} \\
& \hat{\mathrm{v}}(\hat{\lambda})=2 \mathrm{n}^{-1} \lambda^{2}
\end{aligned}
$$

Podemos construir intervalos de confiança (IC) para os parâmetros $\theta$ e $\lambda$ usando a normalidade assintótica dos estimadores de máxima verossimilhança $\dot{\theta}$ e $\hat{\lambda}$ a partir da matriz de informação observada (ou matriz de informaşão de Fisher) dada por,

$$
\begin{aligned}
& \dot{\psi}=(\hat{\theta}, \dot{\lambda})^{\prime} \stackrel{a}{=}\left\{(\theta, \lambda)^{\prime} ; I_{0}^{-1}\right\} \\
& \left(\text { ou } \dot{\psi}=(\hat{\theta}, \hat{\lambda})^{\prime} \stackrel{a}{a}\left\{(\theta, \lambda)^{\prime} ; \mathrm{I}^{-1}(\hat{\psi})\right\}\right)
\end{aligned}
$$

onde $\mathrm{I}_{0}^{-1}$ é a inversa da matriz de informação observada $\left(e \mathrm{I}(\psi)^{-1}\right.$ é a inversa da matriz de informação de Fisher). Portanto, encontramos intervalos de confiança aproximados para os parâmetros (A3.1, Apêndice A3).

Assim, os intervalos de confiança para os parâmetros $\theta$ e $\lambda$, considerando um nivel de confiança $100(1-\gamma) \%$ são dados por, 


$$
\begin{aligned}
& \operatorname{IC}(\theta)=\left[\hat{\theta}-\mathrm{Z}_{\gamma_{/ 2}} \sqrt{\hat{\mathrm{V}}(\dot{\theta})}, \dot{\theta}+\mathrm{Z}_{\gamma_{/ 2}} \sqrt{\hat{\mathrm{V}}(\theta)}\right] \\
& \operatorname{IC}(\lambda)=\left[\dot{\lambda}-\mathrm{Z}_{\gamma_{/ 2}} \sqrt{\hat{\mathrm{V}}(\hat{\lambda})}, \dot{\lambda}+\mathrm{Z}_{\gamma_{/ 2}} \sqrt{\hat{\mathrm{V}}(\lambda)}\right]
\end{aligned}
$$

De maneira análoga, encontramos intervalos de confiança aproximados para $\theta$ e $\lambda$ a partir da matriz de informação de Fisher.

\subsubsection{UMA ANÁLISE BAYESIANA.}

Para este tipo de parametrização consideramos duas densidades a priori: Conjugada natural e Jeffreys. Palmer (1983) observa que nâo existe urna priori conjugada natural para esta parametrização exceto quando $\theta$ é conhecido e $\lambda$ é desconhecido.

\subsubsection{DENSIDADE A PRIORI CONJUGADA NATURAL.}

De (2.1) a f.d.p pode ser escrita na forma,

$$
f(y ; \theta, \lambda)=\left(2 \pi y^{3}\right)^{-1 / 2}\left\{\lambda^{1 / 2} \exp \left[-\lambda\left(\frac{y}{2} \theta^{-2}-\theta^{-1}+\frac{1}{2 y}\right)\right]\right\}
$$

O termo central (Kernel da distribuição) da f.d.p é o termo entre as chaves. Assim se a priori conjugada natural existe sua Densidade a priori conjunta para $\theta$ e $\lambda$ pode ser escrita como,

$$
\pi(\theta, \lambda) \propto \lambda^{d} \exp \left[-\lambda\left(a \theta^{-2}-b \theta^{-1}+c\right)\right]
$$

onde $\theta>0$ e $\lambda>0$.

Dado que $\frac{(y-\theta)^{2}}{2 \theta^{2} y}>0$ é necessario que $a \theta^{-2}-b \theta^{-1}+c>0$, ou seja a $>0$ e $b^{2}-4 a c<0$. A função gama requer também que $d>-1$ para que $\pi(\theta, \lambda)$ seja integrável em relação a $\lambda$.

De (2.26) e (2.27) a Densidade a posteriori conjunta para $\theta$ e $\lambda$ pode ser escrita por,

$$
\pi(\theta, \lambda / \text { dados }) \propto \lambda^{d+n / 2} \exp \left\{-\lambda\left[\left(\frac{\sum y_{i}}{2}+a\right) \theta^{-2}-(n+b) \theta^{-1}+\frac{1}{2} \sum y_{i}^{-1}+c\right]\right\}
$$


2.1 - parametrização $(\theta, \lambda)$ - pág. 15

onde $\theta>0$ e $\lambda>0$; pode-se notar que (2.28) tem a mesma forma de (2.27). Para ver que (2.27) nem sempre existe, consideremos $\lambda$ fixo e $\theta \rightarrow \infty$, assim observamos que $\lim _{\theta \rightarrow \infty} \pi(\theta, \lambda)=$ constante. Portanto, $\pi(\theta, \lambda)$ não é integrável em relação a $\theta$ para algum $\lambda$.

Com $\lambda$ conhecido, a Densidade a priori conjugada natural para $\theta$, se existe, pode ser escrita como,

$$
\pi(\theta) \propto \exp \left\{-\left(a \theta^{-2}-b \theta^{-1}\right)\right]
$$

onde $\theta>0, a>0$ e $b>0$.

Porém, como no caso de ambos os parâmetros serem desconhecidos esta densidade não é própria porque ela não é integrável.

Com $\theta$ conhecido, a Densidade a priori conjugada natural para $\lambda$ é da forma,

$$
\pi(\lambda) \propto \lambda^{\mathrm{d}} \exp [-\lambda \mathrm{k}]
$$

$\operatorname{com~} \mathrm{k}=\mathrm{a} \theta^{-2}-\mathrm{b} \theta^{-1}+\mathrm{c}$, onde $\lambda>0, \mathrm{~d}>0 \mathrm{e} \mathrm{k}>0$.

Pode ser notado ainda que a densidade dada em (2.30) tem a forma da densidade da distribuição gama escrita como,

$$
\pi(\lambda)=\frac{k^{d} \lambda^{d-1} \exp (-k \lambda)}{\Gamma(d)}
$$

onde $d>0, k>0$ e $\lambda>0$.

De (2.26) e (2.30) a Densidade a posteriori marginal para $\lambda$ pode ser escrita como,

$\pi(\lambda /$ dados $) \propto \lambda^{d+n / 2} \exp \left[-\left(k+\frac{\sum\left(y_{i}-\theta\right)^{2}}{2 \theta^{2} y_{i}}\right) \lambda\right]$
onde $\lambda>0, d>0$ e k $>0$.

\subsubsection{DENSIDADE A PRIORI DE JEFFREYS.}

Considerar uma densidade a priori não-informativa de Jeffreys para $\theta$ e $\lambda$ obtida usando a regra de Jeffreys (Box e Tiao, 1973) que é dada por,

$$
\pi(\theta, \lambda) \propto\{\operatorname{det} I(\psi)\}^{1 / 2}
$$

onde I $(\psi)$ é a matriz de informação de Fisher para $\psi=(\theta, \lambda)$. 
De (2.22) temos que,

$$
\operatorname{det} I(\psi)=\left|\begin{array}{cc}
n \lambda \theta^{-3} & 0 \\
0 & \frac{n}{2 \lambda^{2}}
\end{array}\right|=\frac{n^{2} \lambda^{-1} \theta^{-3}}{2}
$$

Substituindo (2.33) em (2.32) encontramos uma Densidade a priori não-informativa de Jeffreys para $\theta$ e d dada por,

$$
\pi(\theta, \lambda) \propto\left\{\lambda \theta^{3}\right\}^{-1 / 2}
$$

onde $\theta>0, \lambda>0$.

Combinando a densidade a priori não-informativa de Jeffreys (2.34) e a função de verossimilhança (2.7), a densidade a posteriori conjunta para $\theta$ e $\lambda$ é dada por (Box e Tiao, 1973),

$$
\pi(\theta, \lambda / \text { dados }) \propto \pi(\theta, \lambda) L(\theta, \lambda)
$$

Substituindo (2.34) e (2.7) em (2.35) obtemos,

$$
\pi(\theta, \lambda / \text { dados }) \propto\left\{\lambda \theta^{3}\right\}^{-1 / 2}(2 \pi)^{-n / 2} \lambda^{n / 2} \prod_{i=1}^{n} y_{i}^{-3 / 2} \exp \{-A(\theta, \lambda)\}
$$

onde $A(\theta, \lambda)=\frac{1}{2} \lambda \theta^{-2} n \bar{Y}-n \lambda \theta^{-1}+\frac{1}{2} \lambda n R$.

Portanto de (2.36) temos que a Densidade a posteriori conjunta para $\theta$ e $\lambda$ é dada por,

$$
\pi(\theta, \lambda / \text { dados }) \propto \theta^{-3 / 2} \lambda^{(n-1) / 2} \exp \{-\mathbf{A}(\theta, \lambda)\}
$$

onde $\theta>0$ e $\lambda>0$.

Palmer (1973) mostra que a expressão (2.37) pode ser normalizada e portanto a densidade a posteriori é própria.

Integrando (2.37) em relação a $\lambda$, obtemos a densidade a posteriori marginal para $\theta$, que é dada por,

$$
\pi(\theta / \text { dados }) \propto \theta^{-3 / 2} \int_{0}^{\infty} \lambda^{(n-1) / 2} \exp \{-\mathbf{A}(\theta, \lambda)\} d \lambda
$$


Para resolver (2.38) usamos alguns resultados de integrais conhecidas (Box e Tiao, 1973, pág. 144) .

Para a $>0, p>0$ temos que,

$$
\int_{0}^{\infty} x^{p-1} \exp \{-a x\} d x=\frac{\Gamma(p)}{a^{p}}
$$

Então escrevendo adequadamente (2.38) temos,

$$
\pi(\theta / \text { dados }) \propto \theta^{-3 / 2} \int_{0}^{\infty} \lambda^{(n-1) / 2} \exp \left\{-\left(\frac{1}{2} \theta^{-2} n \bar{Y}-n \theta^{-1}+\frac{1}{2} n R\right) \lambda\right\} d \lambda
$$

Para resolver essa expressão definimos,

$$
\begin{aligned}
& x=\lambda \\
& p-1=\frac{n-1}{2}, \text { i.e. }, p=\frac{n+1}{2} \\
& a=\left(\frac{1}{2} \theta^{-2} n \bar{Y}-n \theta^{-1}+\frac{1}{2} n R\right)
\end{aligned}
$$

Assim, fazendo uso de (2.39) em (2.40) obtemos,

$$
\pi(\theta / \text { dados }) \propto \theta^{-3 / 2} \frac{\Gamma\left(\frac{\mathrm{n}+1}{2}\right)}{\left[\frac{1}{2} \theta^{-2} \mathrm{n} \overline{\mathrm{Y}}-\mathrm{n} \theta^{-1}+\frac{1}{2} \mathrm{nR}\right]^{(n+1) / 2}}
$$

Portanto, a Densidade a posteriori marginal para $\theta$ é dada por,

$$
\pi(\theta / \text { dados }) \propto \theta^{-3 / 2}\left[\theta^{-2} \mathrm{n} \overline{\mathrm{Y}}-2 \mathrm{n} \theta^{-1}+\mathrm{nR}\right]^{-(n+1) / 2}
$$

onde $\theta>0$.

Analogamente, integrando $(2.37)$ em relação a $\theta$, obtemos a densidade a posteriori marginal para $\lambda$, que é dada por,

$$
\pi(\lambda / \text { dados }) \propto \lambda^{\frac{n-1}{2}} \int_{0}^{\infty} \theta^{-3 / 2} \exp \{-A(\theta, \lambda)\} d \theta
$$

Para resolver a integral em (2.42) usamos o método de Laplace com

$$
f(\theta)=\theta^{-3 / 2} \quad \text { e } \quad h_{\lambda}(\theta)=\frac{1}{2} \lambda \theta^{-2} \bar{Y}-\lambda \theta^{-1}+\frac{1}{2} \lambda R,(\text { ver A.1.1 no apêndice } A 1)
$$


2.1 - parametrizaģão $(\theta, \lambda)$ - pág. 18

Assim, obtemos a Densidade a posteriori marginal para $\lambda$ apraximada pelo método de Laplace,

isto é,

$$
\pi(\lambda / \text { dados }) \propto \lambda^{(n-1) / 2} \frac{\sqrt{2 \pi}}{\sqrt{n}} \frac{1}{(\lambda \bar{Y}-3)^{1 / 2}} \bar{Y}^{-3 / 2} \exp \left\{-n\left(\frac{1}{2} \lambda R-\frac{1}{2} \lambda \bar{Y}^{-1}\right)\right\}
$$

$$
\pi(\lambda / \text { dados }) \propto \lambda^{(n-2) / 2} \exp \left\{-n\left(\frac{1}{2} \lambda R-\frac{1}{2} \lambda \bar{Y}^{-1}\right)\right\}
$$

onde $\lambda>0$.

Considerando $\lambda$ conhecido, em (2.34) a Densidade a priori nāo-informativa de Jeffreys para $\theta$ é dada por,

$$
\pi(\theta) \propto \theta^{-3 / 2}
$$

onde $\theta>0$.

A função de verossimilhança para $\theta$, obtida a partir de (2.7) é dada por,

$$
L(\theta) \propto \exp \left\{-\left(\frac{1}{2} \lambda \theta^{-2} n \bar{Y}-n \lambda \theta^{-1}\right)\right\}
$$

onde $\theta>0$.

$$
\begin{aligned}
& \text { A densidade a posteriori para } \theta \text { é dada por, } \\
& \pi(\theta / \text { dados }) \propto \pi(\theta) L(\theta)
\end{aligned}
$$

Substituindo (2.44) e (2.45) em (2.46) obtemos a Densidade a posteriori para $\theta$ dada por,

$$
\pi(\theta / \text { dados }) \propto \theta^{-3 / 2} \exp \left\{-\left(\frac{1}{2} \lambda \theta^{-2} n \bar{Y}-n \lambda \theta^{-1}\right)\right\}
$$

onde $\theta>0$ e $\lambda$ conhecido.

De forma genérica, a expressão (2.47) pode ser escrita como,

$$
\pi(\theta / \text { dados }) \propto \theta^{-3 / 2} \exp \left(-\mathrm{a} \theta^{-2}+\mathrm{b} \theta^{-1}\right)
$$

onde $\theta>0, a>0$ e $b>0$. 
INTERVALOS DE CREDIBILIDADE PARA OS PARÃMETROS.

Frequentemente usamos a noção de Highest posterior density (HPD) para determinar um intervalo de credibilidade apropriado (ICR) (Box e Tiao, 1973; Zellner, 1981; Press,1989). Assim construimos ICR para os parámetros $\theta$ e $\lambda$ em torno da moda a partir das densidades a posteriori dos parâmetros.

\section{INTERVALO DE CREDIBILIDADE PARA $\theta$.}

Uma aproximação normal para a densidade a posteriori para $\theta$ é dada por,

$$
\pi(\theta / \text { dados }) \stackrel{\sim}{\sim}\left[\hat{\theta},\left(-\left.\frac{\partial^{2} \log \pi(\theta / \text { dados })}{\partial \theta^{2}}\right|_{\theta=\hat{\theta}}\right)^{-1}\right]
$$

onde $\pi$ ( $\theta /$ dados) é dada em (2.41), e $\hat{\theta}$ é a moda da posteriori para $\theta$.

Logo um ICR $100(1-\gamma) \%$ aproximado para $\theta$ é dado por,

$\hat{\theta}-\mathrm{Z}_{\gamma / 2}\left(-\left.\frac{\partial^{2} \log \pi(\theta / \text { dados })}{\partial \theta^{2}}\right|_{\theta=\hat{\theta}}\right)^{-1 / 2}<\theta<\hat{\theta}+\mathrm{Z}_{\gamma / 2}\left(-\left.\frac{\partial^{2} \log \pi(\theta / \text { dados })}{\partial \theta^{2}}\right|_{\theta=\hat{\theta}}\right)^{-1 / 2}$

onde $Z_{\gamma / 2}$ é o percentil de uma distribuição normal padronizada (A3.2, Apéndice A3) e

onde,

$$
\frac{\partial^{2} \log \pi(\theta / \text { dados })}{\partial \theta^{2}}=\frac{3}{2 \theta^{2}}-\left(\frac{n+1}{2}\right)\left(\frac{A^{\prime \prime}(\theta) A(\theta)-\left(A^{\prime}(\theta)\right)^{2}}{A^{2}(\theta)}\right) .
$$

$$
\begin{aligned}
& A(\theta)=\theta^{-2} n \bar{Y}-2 n \theta^{-1}+n R, \\
& A^{\prime}(\theta)=-2 n \bar{Y} \theta^{-3}+2 n \theta^{-2}, \\
& A^{\prime \prime}(\theta)=6 n \bar{Y} \theta^{-4}-4 n \theta^{-3}
\end{aligned}
$$


INTERVALO DE CREDIBILIDADE PARA $\lambda$.

Uma aproximasão normal para a densidade a posteriori para $\lambda$ é dada por,

$$
\pi(\lambda / \text { dados }) \stackrel{a}{\sim}\left[\dot{\lambda},\left(-\left.\frac{\partial^{2} \log \pi(\lambda / \text { dados })}{\partial \lambda^{2}}\right|_{\lambda=\hat{\lambda}}\right)^{-1}\right]
$$

onde $\pi$ ( $\lambda$ / dados) é dada em (2.43), e $\hat{\lambda}$ é a moda da posteriori para $\lambda$.

Logo um ICR $100(1-\gamma) \%$ aproximado para $\lambda$ é dado por,

$\hat{\lambda}-Z_{\gamma / 2}\left(-\left.\frac{\partial^{2} \log \pi(\lambda / \text { dados })}{\partial \lambda^{2}}\right|_{\lambda=\hat{\lambda}}\right)^{-1 / 2}<\lambda<\hat{\lambda}+Z_{\gamma / 2}\left(-\left.\frac{\partial^{2} \log \pi(\lambda / \text { dados })}{\partial \lambda^{2}}\right|_{\lambda=\hat{\lambda}}\right)^{-1 / 2}$

onde $Z_{\gamma / 2}$ é o percentil de uma distribuição normal padronizada, e

$$
\frac{\partial^{2} \log \pi(\lambda / \text { dados })}{\partial \lambda^{2}}=-\left(\frac{n-2}{2}\right)\left(\frac{1}{\lambda^{2}}\right) .
$$

\section{INTERVALO DE CREDIBILIDADE PARA $\theta$ COM $\lambda$ CONHECIDO.}

Uma aproximação normal para a densidade a posteriori para $\theta \operatorname{com} \lambda$ conhecido é dada por,

$$
\pi(\theta / \text { dados }) \stackrel{a}{\sim} N\left[\hat{\theta},\left(-\left.\frac{\partial^{2} \log \pi(\theta / \text { dados })}{\partial \theta^{2}}\right|_{\theta=\hat{\theta}}\right)^{-1}\right]
$$

onde $\pi(\theta /$ dados $)$ é dada em (2.47) e $\hat{\theta}$ é sua moda.

Logo um ICR $100(1-\gamma) \%$ aproximado para $\theta$ é dado por,

$$
\hat{\theta}-Z_{\gamma / 2}\left(-\left.\frac{\partial^{2} \log \pi(\theta / \text { dados })}{\partial \theta^{2}}\right|_{\theta=\hat{\theta}}\right)^{-1 / 2}<\theta<\hat{\theta}+Z_{\gamma / 2}\left(-\left.\frac{\partial^{2} \log \pi(\theta / \text { dados })}{\partial \theta^{2}}\right|_{\theta=\hat{\theta}}\right)^{-1 / 2}
$$

onde $Z_{\gamma / 2}$ é o percentil de uma distribuição normal padronizada, e

$$
\frac{\partial^{2} \log \pi(\theta / \text { dados })}{\partial \theta^{2}}=\frac{3}{2 \theta^{2}}-A^{*}(\theta), \text { onde } A^{*}(\theta)=3 \lambda \theta^{-4} n \bar{Y}-2 n \lambda \theta^{-3} .
$$


2.2. PARAMETRIZAC̄̃̃o $(\mu=1 / \theta, \lambda)$.

Como foi observado na parametrização utilizada na seçāo anterior, nem sempre existe uma densidade a priori própria. Portanto, consideramos a parametrizsça $(\mu=1 / \theta, \lambda)$ que é conveniente para determinar uma priori própria.

\subsubsection{UMA ANÁLISE CLÁSSICA.}

$\mathrm{Na}$ majoria dos casos, é mais fácil trabalhar considerando o inverso da média (ver, Tweedie, 1957). Assim utilizando termos da f.d.p. (2.1) em função de $\theta$ e $\lambda$ temos,

$$
\frac{\lambda}{2 \theta^{2}} \frac{\sum_{i=1}^{n}\left(y_{i}-\theta\right)^{2}}{y_{i}}=\frac{\lambda}{2} \sum_{i=1}^{n}\left(\frac{1}{y_{i}}-\frac{1}{\bar{Y}}\right)+\frac{n \lambda(\bar{Y}-\theta)^{2}}{2 \theta^{2} \bar{Y}}
$$

Se substituirmos (2.51) em (2.1) a f.d.p. pode ser escrita por,

$$
\prod_{i=1}^{n} f(y ; \theta, \lambda)=\left(\frac{\lambda}{2 \pi}\right)^{n / 2} \prod_{i=1}^{n} y_{i}^{-3 / 2} \exp \left\{-\frac{\lambda}{2} \sum_{i=1}^{n}\left(\frac{1}{y_{i}}-\frac{1}{Y}\right)-\frac{n \lambda \bar{Y}}{2}\left(\frac{1}{\theta}-\frac{1}{\bar{Y}}\right)^{2}\right\}
$$

Assim, como $\mu=1 / \theta$ e definindo $v=\sum_{i=1}^{n}\left(\frac{1}{y_{i}}-\frac{1}{\bar{Y}}\right)$ a expressão (2.52) é dada por,

$$
\prod_{i=1}^{n} f(y ; \mu, \lambda)=\left(\frac{\lambda}{2 \pi}\right)^{n / 2} \prod_{i=1}^{n} y_{i}^{-3 / 2} \exp \left\{-\frac{\lambda}{2} v-\frac{n \lambda \bar{Y}}{2}\left(\mu-\frac{1}{\bar{Y}}\right)^{2}\right\}
$$

Portanto, o logaritmo da função de verossimilhança é expressa por,

$$
\mathrm{l}(\mu, \lambda)=\frac{\mathrm{n}}{2} \log \lambda-\frac{\mathrm{n}}{2} \log 2 \pi-\frac{3}{2} \sum_{i=1}^{n} \log y_{i}-\frac{\lambda}{2} v-\frac{\mathrm{n} \lambda \overline{\mathrm{Y}}}{2}\left(\mu-\frac{1}{\overline{\mathrm{Y}}}\right)^{2}
$$

Os EMV de $\mu$ e $\lambda$ podem ser obtidos através das primeiras derivadas de $l(\mu, \lambda)$ com relaçào aos seus parâmetros e igualadas a zero, ou seja,

$$
\begin{aligned}
& \frac{\partial \mathrm{l}(\mu, \lambda)}{\partial \mu}=-\mathrm{n} \lambda \overline{\mathrm{Y}} \mu+\mathrm{n} \lambda \\
& \frac{\partial \mathrm{l}(\mu, \lambda)}{\partial \lambda}=\frac{\mathrm{n} 1}{2 \lambda}-\frac{\mathrm{v}}{2}-\frac{\mathrm{n} \overline{\mathrm{Y}}}{2}\left(\mu-\frac{1}{\bar{Y}}\right)^{2}
\end{aligned}
$$

portanto, 


$$
\begin{aligned}
& \dot{\mu}=\frac{1}{\bar{Y}} \text { e } \\
& \frac{1}{\lambda}=\frac{\mathrm{v}}{\bar{n}}=\frac{1}{\mathrm{~h}} \sum_{i=1}^{n}\left(\frac{1}{y_{i}}-\frac{1}{\bar{Y}}\right) \text { ou } \hat{\lambda}=\frac{n}{\sum_{i=1}^{n}\left(\frac{1}{y_{i}}-\frac{1}{Y}\right)}
\end{aligned}
$$

As inferências para $\psi=(\mu, \lambda)^{\prime}$ podem ser baseadas na aproximação normal assintótica dos estimadores de máxima verossimilhança $\psi=(\hat{\mu}, \hat{\lambda})^{\prime}$ considerando a matriz de informasão observada ou a matriz de informação de Fisher $I(\xi)$ (ver por exemplo, Mood, Graybill e Boes (1974) ou Lawless (1982)), como foi visto na seção 2.1.1.

De (2.12) temos que a matriz de informação observada é dada por,

$$
I_{0}=\left[\begin{array}{cc}
\frac{n^{2} \bar{Y}}{v} & 0 \\
0 & \frac{v^{2}}{2 n}
\end{array}\right]
$$

portanto,

$$
\mathrm{I}_{0}^{-1}=\left[\begin{array}{cc}
\frac{v}{\mathrm{n}^{2} \overline{\bar{Y}}} & 0 \\
0 & \frac{2 n}{v^{2}}
\end{array}\right]
$$

As variâncias aproximadas para $\hat{\mu}$ e $\hat{\lambda}$ são dadas por,

$$
\begin{aligned}
& \hat{\mathrm{V}}(\hat{\mu})=\mathrm{vn}^{-2} \overline{\mathrm{Y}}-1 \\
& \hat{\mathrm{V}}(\hat{\lambda})=2 n v^{-2}
\end{aligned}
$$

Analogamente de (2.19) a matriz de informação de Fisher é dada por,

$$
I(\psi)=\left[\begin{array}{cc}
\frac{\mathrm{n} \lambda}{\mu} & 0 \\
0 & \frac{\mathrm{n}}{2 \lambda^{2}}
\end{array}\right]
$$

portanto, 


$$
I^{-1}(\psi)=\left[\begin{array}{cc}
\frac{\mu}{n \lambda} & 0 \\
0 & \frac{2 \lambda^{2}}{n}
\end{array}\right]
$$

e as variâncias aproximadas para $\hat{\mu}$ e $\hat{\lambda}$ são dadas por,

$$
\begin{aligned}
& \hat{\mathrm{V}}(\hat{\mu})=\mu n^{-1} \lambda^{-1} \\
& \hat{\mathrm{V}}(\hat{\lambda})=2 n^{-1} \lambda^{2}
\end{aligned}
$$

Similarmente à análise feita na seção 2.1.1. podemos construir os intervalos de confiança para os parâmetros $\mu$ e $\lambda$ (A3.1, Apéndice A3).

\subsubsection{UMA ANÁLISE BAYESIANA.}

Para esta parametrização consideramos as seguintes densidades a priori: Conjugada natural, Jeffreys e imprópria.

\subsubsection{DENSIDADE A PRIORI CONJUGADA NATURAL.}

A Densidade a priori conjugada natural para $\mu$ e $\lambda$ tem a forma,

$$
\pi(\mu, \lambda) \propto \lambda^{a} \exp \left\{-\frac{a}{b} \lambda(\mu-b)^{2}-c \lambda\right\}
$$

onde $a>0, b>0$ e $c>0$.

Portanto, esta densidade a priori é conhecida como sendo o produto de uma distribuição normal truncada e uma distribuição gama.

Logo de (2.53) e (2.63) a Densidade a posteriori conjunta para $\mu$ e $\lambda$ pode ser escrita por,

$$
\pi(\mu, \lambda / \text { dados }) \propto \lambda^{a+n / 2} \exp \left\{-n \bar{Y} \lambda\left(\mu-\frac{1}{\bar{Y}}\right)^{2}-v \lambda\right\}
$$


onde $\mu>0$ e $\lambda>0$, e tem a mesma forma de (2.63) com parámetros modificados. 0 desenvolvimento completo é encontrado em Banerjee e Bhattacharyya (1979).

Quando $\lambda$ é conhecido, a Densidade a priori conjugada natural para $\mu$ é dada por,

$$
\pi(\mu) \propto \exp \left\{-\frac{(\mu-c)^{2}}{2 d}\right\}
$$

onde $\mu>0$. Pode ser notado que esta expressão é uma distribuição normal truncada com média c e variancia d. A Densidade a posteriori marginal para $\mu$ tem a mesma forma e admite estimativas baseadas na posteriori da distribuigão normal, ou seja,

$$
\pi(\mu / \text { dados }) \propto \exp \left\{-\frac{\mathrm{n}}{\overline{\mathrm{Y}}}(\mu \overline{\mathrm{Y}}-1)^{2}\right\}
$$

onde $\mu>0$.

Quando $\mu$ é conhecido, a Densidade a priori conjugada natural para $\lambda$ é dada por uma distribuiçāo Gama, e a posteriori tem a mesma forma.

\subsubsection{DENSIDADE A PRIORI DE JEFFREYS.}

Com a parametrização $(\mu, \lambda)$ obtemos a densidade a priori de Jeffreys. A partir da matriz de informação de Fisher (2.60) temos,

$$
\operatorname{det} I(\psi)=\left|\begin{array}{cc}
\frac{n \lambda}{\mu} & 0 \\
0 & \frac{n}{2 \lambda^{2}}
\end{array}\right|=\frac{n^{2}}{2 \mu \lambda}
$$

Substituindo (2.67) em (2.32) temos que a Densidade a priori de Jeffreys para $\mu$ e $\lambda$ é dada por,

$$
\pi(\mu, \lambda) \propto \frac{\mathrm{n}}{\sqrt{\mu \lambda}}
$$

onde $\mu>0$ e $\lambda>0$. 


\subsubsection{DENSIDADE A PRIORJ IMPRÓPRIA.}

Banerjee e Bhattacharyya (1979) consideram a priori imprópria localmente uniforme dada por,

$$
\pi(\mu / \lambda) \propto \text { constante, } \pi(\lambda) \propto \lambda^{-1}
$$

Então de (2.53) e (2.69) temos que a Densidade a posteriori conjunta para $\mu$ e $\lambda$ é dada por,

$$
\pi(\mu, \lambda / \text { dados }) \propto k \lambda^{n / 2-1} \exp \left\{-\frac{n \lambda}{2 \grave{\lambda}}\left[1+\frac{\hat{\lambda}}{\bar{Y}}(\mu \bar{Y}-1)^{2}\right]\right\}
$$

$\operatorname{com} \frac{1}{\lambda}=\frac{1}{n}\left[\sum_{i=1}^{n} \frac{1}{y_{i}}-\frac{n}{\bar{Y}}\right], \bar{Y}=\frac{\sum_{i=1}^{n} y_{i}}{n}$ e $K$ é a constante normalizadora.

Um contôrno de valor constante para $\pi(\mu, \lambda /$ dados $)$ pode ser determinado por, $\pi(\mu, \lambda /$ dados $)=c$, $c>0$.

Assim,

$$
\left(\frac{n}{2}-1\right) \log \lambda-\frac{n \lambda}{2 \hat{\lambda}}\left[1+\frac{\hat{\lambda}}{\bar{Y}}(\mu \bar{Y}-1)^{2}\right]=d, d \text { é uma função de c. }
$$

As modas de $\pi(\mu, \lambda /$ dados $)$ são dadas por,

$$
\frac{\partial \log \pi(\mu, \lambda / \text { dados })}{\partial \mu}=0 \quad \text { e } \frac{\partial \log \pi(\mu, \lambda / \text { dados })}{\partial \lambda}=0
$$

portanto,

$$
\tilde{\mu}=\frac{1}{\bar{Y}} \quad \text { e } \quad \tilde{\lambda}=\frac{(n-2) \hat{\lambda}}{n}
$$

Assim, $-\infty<d<d_{0}$, onde: $d_{0}=\left(\frac{n}{2}-1\right) \log \tilde{\lambda}-\frac{n \tilde{\lambda}}{2 \tilde{\lambda}}\left[1+\frac{\hat{\lambda}}{\overline{\bar{Y}}}(\mu \overline{\mathrm{Y}}-1)^{2}\right]$ Substituindo $\tilde{\mu}$ e $\tilde{\lambda}$ temos, $d_{0}=\left(\frac{n-2}{2}\right)\left[\log \frac{(n-2) \hat{\lambda}}{n}-1\right]$

Quando $n$ é grande, a distribuiçấo de $-2 \log \frac{\pi(\mu, \lambda / \text { dados })}{\pi(\tilde{\mu}, \bar{\lambda} / \text { dados })}$, onde $\tilde{\mu}$ e $\tilde{\lambda}$ são as modas de $\pi(\mu, \lambda /$ dados $)$, é aproximadamente Qui-quadrado com dois graus de liberdade. Baseado nesta distribuição, o contôrno contendo aproximadamente a probabilidade a posteriori $(1-\gamma)$ é o conjunto de pontos dados por,

$$
(n-2) \log \lambda-\frac{n \lambda}{\hat{\lambda}}\left[1+\frac{\hat{\lambda}}{\bar{Y}}(\mu \bar{Y}-1)^{2}\right]=2 d_{0}-\chi_{2, \gamma}^{2}
$$


2.2 - parametrizaşão $(\mu=1 / \theta, \lambda) \cdot$ pág. 26 onde $\chi_{2, \gamma}^{2}$ é a distribuição Qui-quadrado com dois graus de liberdade e $\gamma$ é o nivel de significancia.

Banerjee e Bhattacharyya obtèm outras densidades a posteriori marginais para $\mu$ e $\lambda$.

Integrando (2.70) em relação a $\lambda$ obtemos a densidade a pasteriori marginal para $\mu$ dada por,

$$
\pi(\mu / \text { dados })=\int_{0}^{\infty} k \lambda^{n / 2-1} \exp \left\{-\frac{n \lambda}{2 \dot{\lambda}}\left[1+\frac{\hat{\lambda}}{\bar{Y}}(\mu \bar{Y}-1)^{2}\right]\right\} d \lambda
$$

Para resolver esta integral fazemos,

marginal para $\mu$ é dada por,

$$
p-1=\frac{n}{2}-1, a=\frac{n}{2 \bar{\lambda}}\left[1+\frac{\dot{\lambda}}{\bar{Y}}(\mu \bar{Y}-1)^{2}\right] \text { em (2.39), e portanto, Densidade a posteriori }
$$

$$
\pi(\mu / \text { dados })=k \Gamma\left(\frac{n}{2}\right)\left(\frac{n}{2 \hat{\lambda}}\right)^{-n / 2}\left[1+\hat{\lambda} \bar{Y}\left(\mu-\frac{1}{\bar{Y}}\right)^{2}\right]^{-n / 2}
$$

onde $\mu>0$ e $k=\frac{(\bar{Y} \hat{\lambda})^{1 / 2}(n / 2 \hat{\lambda})^{n / 2}}{S_{n-1}(\zeta) B\left[\frac{(n-1)}{2}, \frac{1}{2}\right] \Gamma\left(\frac{n}{2}\right)}$

com $S_{n-1}(\zeta)$ sendo a F.D.A da distribuição t de student com $(n-1)$ graus de liberdade, e

$$
\zeta=\left[\frac{(n-1) \hat{\lambda}}{\bar{Y}}\right]^{1 / 2}
$$

$$
B(., .) \text { e } \Gamma(\text {. ) são as funçōes beta e gama respetivamente. }
$$

A densidade em (2.74) é uma distribuição $t$ de Student truncada à esquerda com $(n-1)$ graus de liberdade, com parâmetro de locação $1 / \overline{\mathrm{Y}}$, parâmetro de escala $[v \bar{Y} \hat{\lambda}]^{1 / 2}$ e truncada em zero, com $v=(n-1)$.

Integrando $(2.70)$ em relação a $\mu$, obtemos a Densidade a posteriori marginal para $\lambda$ dada por,

$$
\pi(\lambda / \operatorname{dados})=\frac{\Phi\left([n \lambda / \bar{Y}]^{1 / 2}\right)}{S_{n-1}(\zeta)} \frac{(n \lambda / 2 \hat{\lambda})^{(n-1) / 2}}{\lambda \Gamma((n-1) / 2)} \exp \left(-\frac{n \lambda}{2 \bar{\lambda}}\right)
$$

onde $\lambda>0$ e $\Phi$ é a F.D.A da distribuição normal. 
A densidade em (2.75) é uma distribuigão gama multiplicada por um fator envolvendo as F.D.As normal e $t$ de Student, e pode ser definida como uma distribuição gama ponderada. $O$ fator ponderado é dado por,

$$
\frac{\Phi\left((n \lambda / \bar{Y})^{1 / 2}\right)}{S_{n-1}\left([(n-1) \hat{\lambda} / \bar{Y}]^{1 / 2}\right)}
$$

o qual é aproximadamente 1 quando $n \rightarrow \infty$. Assim, em amostras grandes a densidade a posteriori marginal de $\lambda$ tende a uma distribuição Qui-quadrado com $(n-1)$ graus de liberdade.

\section{INTERVALOS DE CREDIBILIDADE PARA OS PARĀMETROS.}

Os intervalos de credibilidade para os parâmetros $\mu$ e $\lambda$ podem ser determinados das densidades a posteriori marginais dadas em (2.74) e (2.75) respectivamente. Estes resultados foram encontrados por Banerjee e Bhattacharyya, 1979.

\section{INTERVALO DE CREDIBILIDADE PARA $\mu$.}

Em particular, o ICR para $\mu$ de (2.74) pode ser determinado notando que no intervalo (0, $2 / \bar{Y})$, a densidade é simétrica e sua moda é $1 / \bar{Y}$.

Portanto, se $\mathrm{P}[\mu \in(1 / \overline{\mathrm{Y}}-\mathrm{a}, 1 / \overline{\mathrm{Y}}+\mathrm{a}) /$ dados $]=1-\gamma$, temos,

$$
a=(\bar{Y} \hat{\lambda} v)^{-1 / 2} S_{n-1}^{-1}\left(\left[1+(1-\gamma) S_{n-1}(\zeta)\right] / 2\right)
$$

Daí, o ICR $(1-\gamma)$ para $\mu$ é dado por,

$$
\frac{1}{\bar{Y}} \pm(\bar{Y} \hat{\lambda} v)^{-1 / 2} S_{n-1}^{-1}\left(\left[1+(1-\gamma) S_{n-1}(\zeta)\right] / 2\right)
$$

com a condição $1 / \bar{Y} \geq$ a. Caso contrario, o ICR $(1-\gamma)$ pode ser obtido considerando $\mathrm{P}[\mu \in(0,1 / \overline{\mathrm{Y}}+\mathrm{b})]=1-\gamma$. Assim novamente de (2.74) temos,

$$
b=(\bar{Y} \hat{\lambda} v)^{-1 / 2} S_{n-1}^{-1}\left(1-\gamma S_{n-1}(\zeta)\right)
$$

Logo, o ICR $(1-\gamma)$ para $\mu$ é dado por,

$$
\left(0, \frac{1}{\bar{Y}}+(\bar{Y} \hat{\lambda} v)^{-1 / 2} S_{n-1}^{-1}\left(1-\gamma S_{n-1}(\zeta)\right)\right)
$$


2.2- parametrizeçāo $(\mu=1 / \theta, \lambda)$ - pág. 28

Os intervalos de credibilidade obtidos em (2.76) e (2.77) são similares em forma aos intervalos de confiança obtidos na análise clássica (Chhikara e Folks, 1976). Isto é esperado já que usamos uma densidade a priori imprópria para obter a densidade a posteriori conjunta para $\mu$ e $\lambda$ (2.70) e suas respectivas densidades marginais (2.74) e (2.75).

Uma outra forma de encontrar intervalos de credibilidade para $\mu$ é através da utilização da aproximação normal. Essa aproximação para a densidade a posteriori para $\mu$ é dada por,

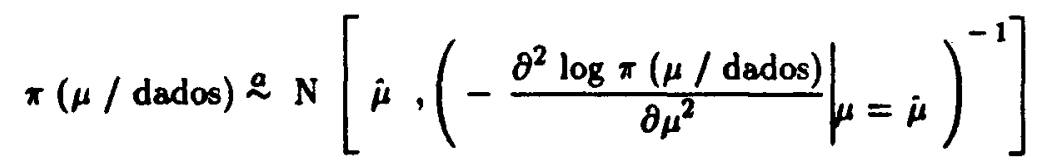

onde $\pi$ ( $\mu$ / dados) é dada em (2.74), e $\hat{\mu}$ é sua moda.

Logo um ICR $100(1-\gamma) \%$ aproximado para $\mu$ é dado por,

$\dot{\mu}-Z_{\gamma / 2}\left(-\left.\frac{\partial^{2} \log \pi(\mu / \text { dados })}{\partial \mu^{2}}\right|_{\mu=\dot{\mu}}\right)^{-1 / 2}<\mu<\hat{\mu}+Z_{\gamma / 2}\left(-\left.\frac{\partial^{2} \log \pi(\mu / \text { dados })}{\partial \mu^{2}}\right|_{\mu=\dot{\mu}}\right)^{-1 / 2}$

onde $\mathrm{Z}_{\gamma / 2}$ é o percentil de uma distribuição normal padronizada (ver Apéndice A3), e

$$
\frac{\partial^{2} \log \pi(\mu / \text { dados })}{\partial \mu^{2}}=-\frac{n}{2}\left(\frac{A^{\prime \prime}(\mu) A(\mu)-\left(A^{\prime}(\mu)\right)^{2}}{A^{2}(\mu)}\right) .
$$

onde,

$$
\begin{aligned}
& A(\mu)=1+\hat{\lambda} \bar{Y}\left(\mu-\frac{1}{\bar{Y}}\right)^{2}, \\
& A^{\prime}(\mu)=2 \mu \hat{\lambda} \bar{Y}-2 \hat{\lambda} \\
& A^{\prime \prime}(\mu)=2 \hat{\lambda} \bar{Y} .
\end{aligned}
$$

\section{INTERVALO DE CREDIBILIDADE PARA $\lambda$.}

Observamos que a densidade (2.75) não é integrável em uma forma exata, portanto não é possivel obter uma expressão explícita para o ICR. Uma solucão numérica pode ser obtida para o ICR $(1-\gamma)$, assim determinamos $\lambda_{I}$ e $\lambda_{L}$, onde $\lambda_{I}$ e $\lambda_{L}$ são determinados tal que, 
2.2 - parametrizasâo $(\mu=1 / \theta, \lambda)$ - pág. 29 $P\left[\lambda \in\left(\lambda_{I}, \lambda_{L}\right) /\right.$ dados $]=1-\gamma$ e $P\left(\lambda_{I} /\right.$ dados $)=P\left(\lambda_{S} /\right.$ dados $)$.

Essa densidade é unimodal e a solução é única. De fato, em amostras grandes, a densidade de $\lambda$ é aproximadamente $(\bar{\lambda} / \mathrm{n}) \chi_{n-1}^{2}$ e o ICR $(1-\gamma)$ de $\lambda$ é dado por,

$$
\left[(\hat{\lambda}) \chi_{n-1,(\gamma / 2)}^{2},\left(\frac{\hat{\lambda}}{\bar{n}}\right) \chi_{n-1,((1-\gamma) / 2)}^{2}\right]
$$

onde $\chi_{n-1}^{2}, \gamma / 2$ e $\chi_{n-1}^{2},(1-\gamma) / 2$ são os pontos percentuais da distribuição Qui-quadrado com $(n-1)$ graus de liberdade. O ICR $(1-\gamma)$ aproximado em (2.79) é o mesmo ao intervalo de confiança obtido na análise clássica (Chhikara e Folks, 1976). 


\section{CAPITULO 3}

\section{UMA ANÁLISE CLÁSSICA PARA O MODELO DE REGRESSÃO LINEAR INVERSO}

\subsection{O MODELO DE REGRESSÃO LINEAR INVERSO.}

A derivação da distribuição Gaussiana Inversa pode ser expressa no contexto do aumento de fadiga do material. Seguindo as idéias desenvolvidas no modelo de falhas com a distribuição de Birnbaum-Saunders (1969), consideramos que um material falha quando acumula fadiga, ou ultrapassa um valor crítico $\omega>0$. 0 tempo de falha $(y)$ também chamado tempo de primeira passagem através de $\omega$, tem distribuição Gaussiana Inversa $\operatorname{IG}(\theta, \lambda) \operatorname{com} \theta=\omega \mu^{-1}$ e $\lambda=\omega^{2} \delta^{-2}$ (ver, Cox e Miller, 1965).

Para uma relação estócastica entre y e a intensidade do estresse $x$, notamos que 0 parámetro $\mu$ é o mais óbvio candidado para ter uma relação direta com $x$ porque mede o aumento da fadiga média por unidade de tempo. Uma forma linear $\mu=\alpha^{\prime}+\beta^{\prime}$ x com a restrição natural $\beta>0$, é uma escolha simples desta relação. De outro lado, as quantidades $\delta$ e $\omega$ correspondem à variabilidade interna do material e o dano crítico que identifica a falha. É razoável supor que essas constantes não sejam relacionadas com $x$. Referindo à (2.1) e absorvendo $\omega$ através dos parâmetros $\alpha$ e $\beta$, temos a estrutura da regressão linear inversa,

$$
\theta^{-1}=\alpha+\beta \mathrm{X}
$$

onde $\alpha \geq 0, \beta \geq 0, \alpha+\beta>0, x>0$.

As restriçōes positivas em (3.1) são devidas ao fato que (2.1) é definida para $\theta>0$. 
Consideramos inicialmente dados sem censuras (dados completos) e $\mathbf{k}$ niveis de uma variável estresse. Colocamos $\mathbf{n}_{i}$ unidades em teste sob condições aceleradas em cada um desses níveis aleatorizados de estresse $x_{i}, i=1,2, \ldots, k$.

Assim, temos as observaçōes $\left(x_{i}, y_{i j}\right), i=1,2, \ldots, k$ e $j=1,2, \ldots, n_{i}$, onde $y_{i j}$ denota o tempo de vida da $j$-ésima observação correspondente a uma variável estresse $x_{i}$. As v.a's $Y_{i 1}, Y_{i 2}$, $\ldots, Y_{i n_{i}}$ são independentes com distribuição $\operatorname{IG}\left(\theta_{i}, \lambda\right) \operatorname{com} \theta_{i}$ dado em (3.1). O experimento pode ser esquematizado na seguinte tabela:

\section{TABELA 3.1}

ESQUEMA DO EXPERIMENTO EM TESTES ACELERADOS

\begin{tabular}{|c|c|c|}
\hline Niveis(k) & Estresses(x) & Tempos de Vida(y) \\
\hline 1 & $x_{1}$ & $y_{11}, y_{12}, \ldots, y_{1 n_{1}}$ \\
2 & $x_{2}$ & $y_{21}, y_{22}, \ldots, y_{2 n_{2}}$ \\
$\cdot$ & $\cdot$ & $\ldots$ \\
$\cdot$ & $\cdot$ & $\ldots$ \\
$\cdot$ & $\cdot$ & $\ldots$ \\
$k$ & $x_{k}$ & $y_{k 1}, y_{k 2}, \ldots, y_{k n_{k}}$ \\
\hline
\end{tabular}

onde $\mathrm{n}=\sum_{i=1}^{k} \mathrm{n}_{i}$ é o número total de observaçōes. 
9.9 - estimadores de máxima verossimilhança - pág. 92

\subsection{ESTIMADORES DE MÁXIMA VEROSSIMILHANÇA.}

Seja $Y_{i 1}, Y_{i 2}, \ldots, Y_{i n_{i}}$ uma a.a. de tamanho $n_{i}$ de uma distribuição Gaussiana Inversa (2.1) com parámetros $\theta_{i}$ e $\lambda$ correspondente a um nivel de estresse $X_{i}$ de um modelo de regressáo linear inverso (3.1), $i=1,2, \ldots, k$. Considerando os dados de $k$ niveis distintos de estresse tomados aleatoriamente, a função de verossimilhança para $\alpha, \beta$ e $\lambda$ é dada por,

$$
\begin{aligned}
& \mathrm{L}(\alpha, \beta, \lambda)=\prod_{i=1}^{k} \prod_{j=1}^{n_{i}}\left(2 \pi \lambda^{-1} y_{i j}^{3}\right)^{-1 / 2} \exp \left\{-\frac{1}{2} \lambda y_{i j}^{-1}\left[y_{i j}\left(\alpha+\beta x_{i}\right)-1\right\}\right\} \\
& =(2 \pi)^{-n / 2} \lambda^{n / 2}\left(\prod_{i=1}^{k} \prod_{j=1}^{n_{i}} y_{i j}^{-3 / 2}\right) \times \\
& \times \exp \left\{-\left(\frac{\lambda \alpha^{2}}{2} \sum_{i=1}^{k} \sum_{j=1}^{n_{i}} y_{i j}+\lambda \alpha \beta \sum_{i=1}^{k} \sum_{j=1}^{n_{i}} y_{i j} x_{i}+\frac{\lambda \beta^{2}}{2} \sum_{i=1}^{k} \sum_{j=1}^{n_{i}} y_{i j} x_{i}^{2}-n \lambda \alpha-\lambda \beta \sum_{i=1}^{k} \sum_{j=1}^{n_{i}} x_{i}+\frac{\lambda}{2} \sum_{i=1}^{k} \sum_{j=1}^{n_{i}} y_{i j}^{-1}\right)\right\}
\end{aligned}
$$

O logaritmo da função de verossimilhança (3.2) é dada por,

$$
\begin{aligned}
\mathrm{l}(\alpha, \beta, \lambda)= & -\frac{\mathrm{n}}{2} \log 2 \pi+\frac{\mathrm{n}}{2} \log \lambda-\frac{3}{2} \sum_{i=1}^{k} \sum_{j=1}^{n_{i}} \log y_{i j}-\frac{\lambda \alpha^{2}}{2} \sum_{i=1}^{k} \sum_{j=1}^{n_{i}} y_{i j}-\lambda \alpha \beta \sum_{i=1}^{k} \sum_{j=1}^{n_{i}} \mathrm{y}_{i j} \mathrm{x}_{i}- \\
& -\frac{\lambda \beta^{2}}{2} \sum_{i=1}^{k} \sum_{j=1}^{n_{i}} \mathrm{y}_{i j} \mathrm{x}_{i}^{2}+\mathrm{n} \lambda \alpha+\lambda \beta \sum_{i=1}^{k} \sum_{j=1}^{n_{i}} \mathrm{x}_{i}-\frac{\lambda}{2} \sum_{i=1}^{k} \sum_{j=1}^{n_{i}} \mathrm{y}_{i j}^{-1}= \\
= & \mathrm{c}(\alpha, \beta, \lambda)-\left\{\frac{\lambda \alpha^{2}}{2} \sum_{i=1}^{k} \sum_{j=1}^{n_{i}} y_{i j}+\lambda \alpha \beta \sum_{i=1}^{k} \sum_{j=1}^{n_{i}} \mathrm{y}_{i j} \mathrm{x}_{i}+\frac{\lambda \beta^{2}}{2} \sum_{i=1}^{k} \sum_{j=1}^{n_{i}} \mathrm{y}_{i j} \mathrm{x}_{i}^{2}+\frac{\lambda}{2} \sum_{i=1}^{k} \sum_{j=1}^{n_{i}} \mathrm{y}_{i j}^{-1}\right\}-\frac{3}{2} \sum_{i=1}^{k} \sum_{j=1}^{n_{i}} \log y_{i j}
\end{aligned}
$$

a qual é definido sobre o espaço paramétrico com as seguintes restrições:

$$
\Omega=\{(\alpha, \beta, \lambda) / \alpha \geq 0, \beta \geq 0, \alpha+\beta>0, \lambda>0\}
$$

A segunda forma de (3.3) mostra que temos uma familia exponencial de distribuições com quatro estatísticas suficientes dadas por,

$$
\sum_{i=1}^{k} \sum_{j=1}^{n_{i}} y_{i j}, \sum_{i=1}^{k} \sum_{j=1}^{n_{i}} y_{i j} x_{i}, \sum_{i=1}^{k} \sum_{j=1}^{n_{i}} y_{i j} x_{i}^{2}, \sum_{i=1}^{k} \sum_{j=1}^{n_{i}} y_{i j}^{-1} .
$$


9.9 - estimadores de máxima verossimilhança - pág. 99

Seja $\psi=(\alpha, \beta, \lambda)^{\prime}$ o vetor de estimativas e consideremos a maximizaçāo de $1(\psi)$ com respeito a $\psi$. As primeiras derivadas de $\mathrm{l}(\psi)(3.3)$ em relação a $\alpha, \beta$ e $\lambda$ são dadas por,

$$
\begin{array}{r}
\frac{\partial 1(\psi)}{\partial \alpha}=-\lambda \alpha \sum_{i=1}^{k} \sum_{j=1}^{n_{i}} y_{i j}-\lambda \beta \sum_{i=1}^{k} \sum_{j=1}^{n_{i}} y_{i j} x_{i}+n \lambda= \\
=\lambda \sum_{i=1}^{k} \sum_{j=1}^{n_{i}}\left[1-\left(\alpha+\beta x_{i}\right) y_{i j}\right]=0
\end{array}
$$

$$
\begin{gathered}
\frac{\partial 1(\psi)}{\partial \beta}=-\lambda \alpha \sum_{i=1}^{k} \sum_{j=1}^{n_{i}} y_{i j} x_{i}-\lambda \beta \sum_{i=1}^{k} \sum_{j=1}^{n_{i}} y_{i j} x_{i}^{2}+\lambda \sum_{i=1}^{k} \sum_{j=1}^{n_{i}} x_{i}= \\
=\lambda \sum_{i=1}^{k} \sum_{j=1}^{n_{i}} x_{i}\left[1-\left(\alpha+\beta x_{i}\right) y_{i j}\right]=0
\end{gathered}
$$

$$
\begin{gathered}
\frac{\partial 1(\psi)}{\partial \lambda}=\frac{\mathrm{n}}{2 \lambda} \\
-\frac{\alpha^{2}}{2} \sum_{i=1}^{k} \sum_{j=1}^{n_{i}} y_{i j}-\alpha \beta \sum_{i=1}^{k} \sum_{j=1}^{n_{i}} y_{i j} x_{i}-\frac{\beta^{2}}{2} \sum_{i=1}^{k} \sum_{j=1}^{n_{i}} y_{i j} x_{i}^{2}+n \alpha+\beta \sum_{i=1}^{k} \sum_{j=1}^{n_{i}} x_{i}-\frac{1}{2} \sum_{i=1}^{k} \sum_{j=1}^{n_{i}} y_{i j}^{-1}= \\
=\frac{n}{2 \lambda}-\frac{1}{2} \sum_{i=1}^{k} \sum_{j=1}^{n_{i}} y_{i j}^{-1}\left[1-\left(\alpha+\beta x_{i}\right) y_{i j}\right]^{2}=0
\end{gathered}
$$

A seguir definimos as seguintes estatisticas básicas:

$$
\begin{aligned}
& \mathrm{V}_{l}=\mathrm{n}^{-1} \sum_{i=1}^{k} \sum_{j=1}^{n_{i}} \mathrm{y}_{i j} \mathrm{x}_{i}^{l}, \mathrm{l}=0,1,2 . \\
& \overline{\mathrm{X}}=\mathrm{n}^{-1} \sum_{i=1}^{k} \sum_{j=1}^{n_{i}} \mathrm{x}_{i} \\
& \overline{\mathrm{Y}}=\mathrm{n}^{-1} \sum_{i=1}^{k} \sum_{j=1}^{n_{i}} \mathrm{y}_{i j} \\
& \mathrm{R}=\mathrm{n}^{-1} \sum_{i=1}^{k} \sum_{j=1}^{n_{i}} \mathrm{y}_{i j}^{-1}
\end{aligned}
$$


$\$ .9$ - estimadores de máxima verossimithança - pág. $\$ 4$

Da equação (3.4) e (3.5) temos respectivamente,

$$
\begin{aligned}
& \lambda\left(1-\alpha V_{0}-\beta V_{1}\right)=0, \\
& \lambda\left(\bar{X}-\alpha V_{1}-\beta V_{2}\right)=0,
\end{aligned}
$$

Assim, como $\lambda>0$ obtemos,

$$
\begin{aligned}
& \alpha V_{0}+\beta V_{1}=1 \\
& \alpha V_{1}+\beta V_{2}=\bar{X}
\end{aligned}
$$

onde as equações (3.8) são lineares nos parâmetros.

Para obtermos as estimativas dos parâmetros necessitamos utilizar as informaçōes das equações anteriores, para isso pela equação (3.4), como $\lambda>0$ temos,

$$
\mathrm{n}-\alpha \sum_{i=1}^{k} \sum_{j=1}^{n_{i}} y_{i j}-\beta \sum_{i=1}^{k} \sum_{j=1}^{n_{i}} y_{i j} x_{i}=0
$$

portanto, $\alpha=\frac{n-\beta \sum_{i=1}^{k} \sum_{j=1}^{n_{i}} y_{i j} x_{i}}{\sum_{i=1}^{k} \sum_{j=1}^{n_{i}} y_{i j}}$

e da equação (3.5), como $\lambda>0$ temos,

$$
\sum_{i=1}^{k} \sum_{j=1}^{n_{i}} x_{i}-\alpha \sum_{i=1}^{k} \sum_{j=1}^{n_{i}} y_{i j} x_{i}-\beta \sum_{i=1}^{k} \sum_{j=1}^{n_{i}} y_{i j} x_{i}^{2}=0
$$

portanto,

$$
\alpha=\frac{\sum_{i=1}^{k} \sum_{j=1}^{n_{i}} x_{i}-\beta \sum_{i=1}^{k} \sum_{j=1}^{n_{i}} y_{i j} x_{i}^{2}}{\sum_{i=1}^{k} \sum_{j=1}^{n_{i}} y_{i j} x_{i}},
$$

Assim, a estimativa de $\beta$ pode ser obtida igualando as expressões (3.9) e (3.10) da seguinte forma,

$$
\frac{n-\beta \sum_{i=1}^{k} \sum_{j=1}^{n_{i}} y_{i j} x_{i}}{\sum_{i=1}^{k} \sum_{j=1}^{n_{i}} y_{i j}}=\frac{\sum_{i=1}^{k} \sum_{j=1}^{n_{i}} x_{i}-\beta \sum_{i=1}^{k} \sum_{j=1}^{n_{i}} y_{i j} x_{i}^{2}}{\sum_{i=1}^{k} \sum_{j=1}^{n_{i}} y_{i j} x_{i}}
$$


9.9 - estimadores de máxima verossimilhanģa - pág. 95

portanto,

$$
\hat{\beta}=\frac{\sum_{i=1}^{k} \sum_{j=1}^{n_{i}} y_{i j} \sum_{i=1}^{k} \sum_{j=1}^{n_{i}} x_{i}-n \sum_{i=1}^{k} \sum_{j=1}^{n_{i}} y_{i j} x_{i}}{\sum_{i=1}^{k} \sum_{j=1}^{n_{i}} y_{i j} \sum_{i=1}^{k} \sum_{j=1}^{n_{i}} y_{i j} x_{i}^{2}-\left(\sum_{i=1}^{k} \sum_{j=1}^{n_{i}} y_{i j} x_{i}\right)^{2}} .
$$

A estimativa de $\alpha$ é dada substituindo a expressão (3.11) em (3.10) ou (3.9), ou seja,

$$
\hat{\alpha}=\frac{n \sum_{i=1}^{k} \sum_{j=1}^{n_{i}} y_{i j} x_{i}^{2}-\sum_{i=1}^{k} \sum_{j=1}^{n_{i}} x_{i} \sum_{i=1}^{k} \sum_{j=1}^{n_{i}} y_{i j} x_{i}}{\sum_{i=1}^{k} \sum_{j=1}^{n_{i}} y_{i j} \sum_{i=1}^{k} \sum_{j=1}^{n_{i}} y_{i j} x_{i}^{2}-\left(\sum_{i=1}^{k} \sum_{j=1}^{n_{i}} y_{i j} x_{i}\right)^{2}} .
$$

A estimativa de $\lambda$ pode ser obtida diretamente da equação (3.6), ou seja,

$$
\hat{\lambda}^{-1}=\hat{\alpha}^{2} \mathrm{n}^{-1} \sum_{i=1}^{k} \sum_{j=1}^{n_{i}} \mathrm{y}_{i j}+2 \hat{\alpha} \hat{\beta} \mathrm{n}^{-1} \sum_{i=1}^{k} \sum_{j=1}^{n_{i}} \mathrm{y}_{i j} \mathrm{x}_{i}+\dot{\beta}^{2} \mathrm{n}^{-1} \sum_{i=1}^{k} \sum_{j=1}^{n_{i}} y_{i j} \mathrm{x}_{i}^{2}-2 \hat{\alpha}-2 \hat{\beta}^{-1} \sum_{i=1}^{k} \sum_{j=1}^{n_{i}} \mathrm{x}_{i}+\mathrm{n}^{-1} \sum_{i=1}^{k} \sum_{j=1}^{n_{i}} y_{i j}^{-1}
$$

Logo, o vetor de estimativas $\hat{\psi}_{L}=\left(\hat{\alpha}_{L}, \hat{\beta}_{L}, \hat{\lambda}_{L}\right)^{\prime}$ é dado por (3.11), (3.12) e (3.13), que podem ser escritos em termos das estatisticas básicas (3.7). Definindo $D \equiv V_{0} V_{2}-V_{1}^{2}>0$ com probabilidade um (pela desigualdade de Cauchy-Schwarz) temos,

$$
\mathrm{D} \equiv \mathrm{V}_{0} \mathrm{~V}_{2}-\mathrm{V}_{1}^{2}=\mathrm{n}^{-1} \sum_{i=1}^{k} \sum_{j=1}^{n_{i}} y_{i j} \mathrm{n}^{-1} \sum_{i=1}^{k} \sum_{j=1}^{n_{i}} y_{i j} \mathrm{x}_{i}^{2}-\left[\mathrm{n}^{-1} \sum_{i=1}^{k} \sum_{j=1}^{n_{i}} y_{i j} x_{i}\right]^{2}
$$

Isto é, $\mathrm{D}=\frac{\left[\sum_{i=1}^{k} \sum_{j=1}^{n_{i}} y_{i j} \sum_{i=1}^{k} \sum_{j=1}^{n_{i}} y_{i j} x_{i}^{2}-\left[\sum_{i=1}^{k} \sum_{j=1}^{n_{i}} y_{i j} x_{i}\right]^{2}\right]}{n^{2}}$

e $\quad \mathrm{D}^{-1}=\frac{\mathrm{n}^{2}}{\left[\sum_{i=1}^{k} \sum_{j=1}^{n_{i}} y_{i j} \sum_{i=1}^{k} \sum_{j=1}^{n_{i}} y_{i j} \mathrm{x}_{i}^{2}-\left[\sum_{i=1}^{k} \sum_{j=1}^{n_{i}} \mathrm{y}_{i j} \mathbf{x}_{i}\right]^{2}\right]}$ 
Portanto, temos,

$$
\begin{aligned}
& \hat{\alpha}_{L}=\frac{\mathrm{V}_{2}-\overline{\mathrm{X}} \mathrm{V}_{1}}{\mathrm{~V}_{0} \mathrm{~V}_{2}-\mathrm{V}_{1}^{2}}=\left(\mathrm{V}_{2}-\overline{\mathrm{X}} \mathrm{V}_{1}\right) D^{-1}=\overline{\mathrm{Y}}^{-1}\left(1-\mathrm{V}_{1} \dot{\beta}_{L}\right) \\
& \hat{\beta}_{L}=\frac{\overline{\mathrm{X}} \mathrm{V}_{0}-\mathrm{V}_{1}}{\mathrm{~V}_{0} \mathrm{~V}_{2}-\mathrm{V}_{1}^{2}}=\left(\overline{\mathrm{X}} \mathrm{V}_{0}-\mathrm{V}_{1}\right) D^{-1}=-(\mathrm{nD})^{-1} \sum_{i=1}^{k} \sum_{j=1}^{n_{i}}\left(\mathrm{x}_{i}-\overline{\mathrm{X}}\right)\left(\mathrm{y}_{i j}-\overline{\mathrm{Y}}\right) \\
& \hat{\lambda}_{L}^{-1}=\dot{\alpha}_{L}^{2} \mathrm{~V}_{0}+2 \hat{\alpha}_{L} \hat{\beta}_{L} \mathrm{~V}_{1}+\dot{\beta}_{L}^{2} \mathrm{~V}_{2}-2 \hat{\alpha}_{L}-2 \hat{\beta}_{L} \overline{\mathrm{X}}+\mathrm{R}
\end{aligned}
$$

De (3.8) e da equação anterior, escrevendo a expressão em forma adequada temos,

$$
\hat{\lambda}_{L}^{-1}=\mathbf{R}-\hat{\alpha}_{L}-\dot{\beta}_{L} \overline{\mathrm{X}}=\mathrm{n}^{-1} \sum_{i=1}^{k} \sum_{j=1}^{n_{i}}\left(\mathrm{y}_{i j}^{-1}-\hat{\alpha}_{L}-\hat{\beta}_{L} \mathbf{x}_{i}\right)
$$

Observamos que $\hat{\beta}_{L}$ envolve o termo da covariancia usual no numerador mas o seu denominador é quadrático em y. Podemos chamar $\hat{\psi}_{L}=\left(\hat{\alpha}_{L}, \hat{\beta}_{L}, \hat{\lambda}_{L}\right)$ como as raizes das equaçōes de máxima verossimilhança (REMV).

Para a obtenção dos estimadores de máxima verossimilhança (EMV) é necessario examinar se as REMV se encontram ou não no espaço paramétrico. Battacharyya e Fries (1981) estabelecem uma caracterização formal dos EMV $\dot{\psi}$ dados por,

$$
(\hat{\alpha}, \hat{\beta})=\left\{\begin{array}{l}
\left(\dot{\alpha}_{L}, \hat{\beta}_{L}\right) \text { se } \mathrm{V}_{1}<\min \left(\overline{\mathrm{X}} \mathrm{V}_{0}, \overline{\mathrm{X}}^{-1} \mathrm{~V}_{2}\right) \\
\left(0, \overline{\mathrm{X}} \mathrm{V}_{2}^{-1}\right) \text { se } \overline{\mathrm{X}}^{-1} \mathrm{~V}_{2} \leq \mathrm{V}_{1}<\overline{\mathrm{X}} \mathrm{V}_{0} \\
\left(\mathrm{~V}_{0}^{-1}, 0\right) \text { se } \overline{\mathrm{X}} \mathrm{V}_{0} \leq \mathrm{V}_{1}<\overline{\mathrm{X}}^{-1} \mathrm{~V}_{2}
\end{array}\right.
$$

e $\hat{\lambda}^{-1}=\mathrm{R}-\hat{\alpha}-\hat{\beta} \bar{X}$, onde $\hat{\alpha}_{L}$ e $\hat{\beta}_{L}$ são dados em (3.14) e (3.15). 


\subsection{INFERÉNCLAS SOBRE OS PARÁMETROS.}

Como foi visto no capítulo 2, as inferências sobre os parâmetros sāo usualmente baseadas na distribuição normal limite dos EMV considerando a matriz de informação observada ou a matriz de informação de Fisher (ver por exemplo, Mood, Graybill e Boes, 1974; Lawless, 1982).

As segundas derivadas parciais com sinal negativo da função log-verossimilhança (3.3) com respeito à $\alpha, \beta, \lambda$ são dadas por,

$$
\begin{aligned}
& -\frac{\partial^{2} \mathrm{l}(\psi)}{\partial \alpha^{2}}=\lambda \sum_{i=1}^{k} \sum_{j=1}^{n_{i}} \mathrm{y}_{i j}=\mathrm{n} \lambda \mathrm{V}_{0} \\
& -\frac{\partial^{2} \mathrm{l}(\psi)}{\partial \beta^{2}}=\lambda \sum_{i=1}^{k} \sum_{j=1}^{n_{i}} \mathrm{y}_{i j} \mathrm{x}_{i}^{2}=\mathrm{n} \lambda \mathrm{V}_{2} \\
& -\frac{\partial^{2} \mathrm{l}(\psi)}{\partial \lambda^{2}}=\frac{\mathrm{n}}{2 \lambda^{2}} \\
& -\frac{\partial^{2} \mathrm{l}(\psi)}{\partial \alpha \partial \beta}=\lambda \sum_{i=1}^{k} \sum_{j=1}^{n_{i}} \mathrm{y}_{i j} \mathrm{x}_{i}=\mathrm{n} \lambda \mathrm{V}_{1} \\
& -\frac{\partial^{2} \mathrm{l}(\psi)}{\partial \alpha \partial \lambda}=\alpha \sum_{i=1}^{k} \sum_{j=1}^{n_{i}} \mathrm{y}_{i j}+\beta \sum_{i=1}^{k} \sum_{j=1}^{n_{i}} \mathrm{y}_{i j} \mathrm{x}_{i}-\mathrm{n}=\alpha \mathrm{n} \mathrm{V}_{0}+\mathrm{n} \beta \mathrm{V}_{1}-\mathrm{n} \\
& -\frac{\partial^{2} \mathrm{l}(\psi)}{\partial \beta \partial \lambda}=\alpha \sum_{i=1}^{k} \sum_{j=1}^{n_{i}} \mathrm{y}_{i j} \mathrm{x}_{i}+\beta \sum_{i=1}^{k} \sum_{j=1}^{n_{i}} \mathrm{y}_{i j} \mathrm{x}_{i}^{2}-\sum_{j=1}^{k} \sum_{i=1}^{n_{j}} \mathrm{x}_{i}=\mathrm{n} \alpha \mathrm{V}_{1}+\mathrm{n} \beta \mathrm{V}_{2}-\mathrm{n} \overline{\mathrm{X}}
\end{aligned}
$$

Assim, a matriz de informação observada $\mathrm{I}_{0}$ (de 2.12) é dada por,

$$
I_{0}=\left[\begin{array}{ccc}
n \hat{\lambda} V_{0} & n \hat{\lambda} V_{1} & n \hat{\alpha} V_{0}+n \hat{\beta} V_{1}-n \\
n \hat{\lambda} V_{1} & n \hat{\lambda} V_{2} & n \hat{\alpha} V_{1}+n \hat{\beta} V_{2}-n \bar{X} \\
n \hat{\alpha} V_{0}+n \hat{\beta} V_{1}-n & n \hat{\alpha} V_{1}+n \hat{\beta} V_{2}-n \bar{X} & \frac{n}{2 \hat{\lambda}^{2}}
\end{array}\right]
$$


Usando (3.8) a matriz de informasaa observada $I_{0}$ pode ser escrita na forma,

$$
I_{0}=\left[\begin{array}{ccc}
n \hat{\lambda} V_{0} & n \hat{\lambda} V_{1} & 0 \\
n \hat{\lambda} V_{1} & n \hat{\lambda} V_{2} & 0 \\
0 & 0 & \frac{n}{2 \hat{\lambda}^{2}}
\end{array}\right]
$$

Para fazer inferências para $\psi=(\alpha, \beta, \lambda)^{\prime}$, usamos o fato que para n grande,

$$
\dot{\psi}=(\hat{\alpha}, \hat{\beta}, \hat{\lambda})^{\prime} \stackrel{a}{\sim} \mathrm{N}\left\{(\alpha, \beta, \lambda)^{\prime}, \mathrm{I}_{0}^{-1}\right\}
$$

A estrutura bloco diagonal de (3.25) nos leva à,

$$
I_{0}^{-1}=\left[\begin{array}{ccc}
\frac{V_{2}}{n \hat{\lambda} D} & -\frac{V_{1}}{n \hat{\lambda} D} & 0 \\
-\frac{V_{1}}{n \hat{\lambda} D} & \frac{V_{0}}{n \hat{\lambda} D} & 0 \\
0 & 0 & \frac{2 \hat{\lambda}^{2}}{n}
\end{array}\right]
$$

Assim as variâncias aproximadas para $\hat{\alpha}, \hat{\beta}, \hat{\lambda}$ e $\hat{\lambda}^{-1}$ são dadas por,

$$
\begin{aligned}
& \hat{\mathrm{V}}(\hat{\alpha}) \approx(n \hat{\lambda} D)^{-1} V_{2} \\
& \hat{\mathrm{V}}(\hat{\beta}) \approx(n \bar{\lambda} D)^{-1} V_{0} \\
& \hat{\mathrm{V}}(\hat{\lambda}) \approx 2 n^{-1} \hat{\lambda}^{2}
\end{aligned}
$$

e para calcular $\hat{V}\left(\hat{\lambda}^{-1}\right)$ usamos o método Delta (Miller, 1981). Assim,

$$
\hat{\lambda} \stackrel{\mathrm{a}}{N}\{\lambda, \hat{\mathrm{V}}(\hat{\lambda})\} \text { ou } \dot{\lambda} \stackrel{a}{N}\left\{\lambda, 2 \mathrm{n}^{-1} \bar{\lambda}^{2}\right\}
$$

Definindo $g(\hat{\lambda})=\frac{1}{\hat{\lambda}}$, temos $g(\hat{\lambda}) \stackrel{a}{\sim} N\left\{g(\lambda), \hat{V}(\hat{\lambda})\left(g^{\prime}(\hat{\lambda})\right)^{2}\right\}$

Dai, $g^{\prime}(\hat{\lambda})=-\frac{1}{\hat{\lambda}^{2}}$, isto $\dot{e}, \hat{\lambda}^{-1} \stackrel{\mathrm{g}}{=}\left\{\lambda^{-1}, 2 \mathrm{n}^{-1} \hat{\lambda}^{-2}\right\}$ 
Portanto,

$$
\hat{\mathbf{v}}\left(\hat{\lambda}^{-1}\right) \approx 2 n^{-1} \dot{\lambda}^{-2}
$$

A matriz de informação de Fisher (de (2.19)) è dada por,

$$
I(\psi)=E\left[\begin{array}{ccc}
n \lambda V_{0} & n \lambda V_{1} & 0 \\
n \lambda V_{1} & n \lambda V_{2} & 0 \\
0 & 0 & \frac{n}{2 \lambda^{2}}
\end{array}\right]
$$

onde, $v_{l}=n^{-1} \sum_{i=1}^{k} \sum_{j=1}^{n_{i}} y_{i j} x_{i}^{l}, \quad l=0,1,2(3.7)$

Como, $E\left(V_{l}\right)=n^{-1} \sum_{i=1}^{k} \sum_{j=1}^{n_{i}} x_{i}^{l} E\left(y_{i j}\right)$, onde $E\left(y_{i j}\right)=\theta_{i}=\left(\alpha+\beta x_{i}\right)^{-1}$, temos $E\left(V_{l}\right)=n^{-1} \sum_{i=1}^{k} \sum_{j=1}^{n_{i}} x_{i}^{l}\left(\alpha+\beta x_{i}\right)^{-1}, \quad l=0,1,2$.

Definindo, $\mathrm{W}_{l}(\alpha, \beta)=\mathrm{E}\left(\mathrm{v}_{l}\right)=\mathrm{n}^{-1} \sum_{i=1}^{k} \sum_{j=1}^{n_{i}} \mathrm{x}_{i}^{l}\left(\alpha+\beta \mathrm{x}_{i}\right)^{-1}, l=0,1,2$ e $\mathrm{E} \equiv \mathrm{W}_{0} \mathrm{~W}_{2}-\mathrm{W}_{1}^{2}>0$, temos,

$$
\mathrm{I}(\psi)=\left[\begin{array}{ccc}
\mathrm{n} \lambda \mathrm{W}_{0}(\alpha, \beta) & \mathrm{n} \lambda \mathrm{W}_{1}(\alpha, \beta) & 0 \\
\mathrm{n} \lambda \mathrm{W}_{1}(\alpha, \beta) & \mathrm{n} \lambda \mathrm{W}_{2}(\alpha, \beta) & 0 \\
0 & 0 & \frac{\mathrm{n}}{2 \lambda^{2}}
\end{array}\right]
$$

De (3.33), $\mathrm{I}^{-1}(\psi)$ é dada por,

$$
I^{-1}(\psi)=\left[\begin{array}{ccc}
\frac{W_{2}(\alpha, \beta)}{n \lambda E} & -\frac{W_{1}(\alpha, \beta)}{n \lambda E} & 0 \\
-\frac{W_{1}(\alpha, \beta)}{n \lambda E} & \frac{W_{0}(\alpha, \beta)}{n \lambda E} & 0 \\
0 & 0 & \frac{2 \lambda^{2}}{n}
\end{array}\right]
$$

e as variâncias aproximadas para $\hat{\alpha}, \hat{\beta}, \hat{\lambda}$ e $\hat{\lambda}^{-1}$ são dadas por, 
$\dot{v}(\hat{a}) \approx(n \lambda E)^{-1} W_{2}(\alpha, \beta)$

$\hat{\mathrm{V}}(\hat{\beta}) \approx(\mathrm{n} \lambda \mathrm{E})^{-1} \mathrm{~W}_{0}(\alpha, \beta)$

$\hat{\mathrm{V}}(\hat{\lambda}) \approx 2 \mathrm{n}^{-1} \lambda^{2}$

$\hat{\mathrm{V}}\left(\hat{\lambda}^{-1}\right) \approx 2 \mathrm{n}^{-1} \lambda^{-2} \quad($ pelo Método Delta $)$

$O$ inverso do tempo médio de falha $\theta^{-1}=\alpha+\beta x^{*}$, num nivel de estresse especificado $x^{*}$, é estimado por, $\hat{\theta}^{-1}=\hat{\alpha}+\dot{\beta} x^{*}$, cuja variância estimada aproximada é dada por,

$\hat{\mathrm{V}}\left(\hat{\theta}^{-1}\right) \approx(\mathrm{nD} \hat{\lambda})^{-1}\left(\mathrm{~V}_{2}-2 \mathrm{x}^{*} \mathrm{~V}_{1}+\mathrm{x}^{*} \mathrm{~V}_{0}\right), \mathrm{D} \equiv \mathrm{V}_{0} \mathrm{~V}_{2}-\mathrm{V}_{1}^{2}>0$ (pelo Método Delta)

Isto é verificado como segue: observar que,

Então,

$$
\left[\begin{array}{c}
\hat{\alpha} \\
\hat{\beta}
\end{array}\right] \approx \mathrm{N}\left\{\left[\begin{array}{l}
\alpha \\
\beta
\end{array}\right] ;\left[\begin{array}{cc}
\hat{\mathrm{V}}(\hat{\alpha}) & \operatorname{cov}(\hat{\alpha}, \hat{\beta}) \\
\operatorname{cov}(\hat{\alpha}, \hat{\beta}) & \hat{\mathrm{V}}(\hat{\beta})
\end{array}\right]\right\}
$$

$$
\left[\begin{array}{c}
\hat{\alpha} \\
\hat{\beta}
\end{array}\right] \stackrel{\sim}{\sim}\left\{\left[\begin{array}{l}
\alpha \\
\beta
\end{array}\right] ;\left[\begin{array}{cc}
(\mathrm{n} \hat{\lambda} D)^{-1} V_{2} & -(n \hat{\lambda} D)^{-1} V_{1} \\
-(n \hat{\lambda} D)^{-1} V_{1} & (n \hat{\lambda} D)^{-1} V_{0}
\end{array}\right]\right\}
$$

Definindo $g(\hat{\alpha}, \hat{\beta})=\hat{\theta}^{-1}=\hat{\alpha}+\dot{\beta} x^{*}$, temos

$$
\mathrm{g}(\hat{\alpha}, \hat{\beta}) \stackrel{a}{\sim} \mathrm{N}\left\{\mathrm{g}(\alpha, \beta) ;\left(\hat{\mathrm{V}}(\hat{\alpha})\left(\frac{\partial \mathrm{g}(\hat{\alpha}, \hat{\beta})}{\partial \hat{\alpha}}\right)^{2}+2 \operatorname{cov}(\hat{\alpha}, \hat{\beta}) \frac{\partial \mathrm{g}(\dot{\alpha}, \hat{\beta})}{\partial \dot{\alpha}} \frac{\partial \mathrm{g}(\hat{\alpha}, \hat{\beta})}{\partial \hat{\beta}}+\dot{\mathrm{V}}(\hat{\beta})\left(\frac{\partial \mathrm{g}(\hat{\alpha}, \hat{\beta})}{\partial \hat{\beta}}\right)^{2}\right)\right\}
$$

Como, $\frac{\partial \mathrm{g}(\hat{\alpha}, \hat{\beta})}{\partial \hat{\alpha}}=1, \quad \frac{\partial \mathrm{g}\left(\hat{\alpha}_{;}, \hat{\beta}\right)}{\partial \hat{\beta}}=\mathrm{x}^{*}$, então

$$
\hat{\theta}^{-1} \stackrel{a}{\sim} \mathrm{N}\left\{g(\alpha, \beta) ;\left(\frac{V_{2}}{n \hat{\lambda} D}+2\left(-\frac{V_{1}}{n \hat{\lambda} D}\right) x^{*}+\frac{V_{0}}{n \hat{\lambda} D}\left(x^{*}\right)^{2}\right)\right\}
$$


isto é,

$$
\hat{\theta}^{-1} \stackrel{N}{\sim}\left\{g(\alpha, \beta) ;\left[(n \hat{A} D)^{-1}\left(v_{2}-2 v_{1} x^{*}+V_{0} x^{* 2}\right)\right]\right\}
$$

Portanto,

$$
\hat{\mathrm{V}}\left(\hat{\theta}^{-1}\right) \approx(\mathrm{n} \dot{\lambda} D)^{-1}\left(V_{2}-2 V_{1} x^{*}+V_{0} x^{*}\right)
$$

\subsection{INTERVALOS DE CONFLANÇA PARA OS PARÀMETROS.}

Nosso interesse é construir intervalos de confiança para os parámetros $\alpha, \beta, \lambda, \lambda^{-1}$. Para isso usamos a normalidade assintótica dos estimadores de máxima verossimilhança $\hat{\alpha}, \hat{\beta}, \hat{\lambda}$, e $\hat{\lambda}^{-1}$ a partir da matriz de informação observada ( ou matriz de informação de Fisher) dado por,

$$
\begin{aligned}
& \dot{\psi}=(\hat{\alpha}, \dot{\beta}, \hat{\lambda})^{\prime} \stackrel{a}{\mathrm{~N}}\left\{(\alpha, \beta, \lambda)^{\prime} ; \mathrm{I}_{0}^{-1}(\psi)\right\} \\
& \left(\text { ou } \hat{\psi}=(\hat{\alpha}, \hat{\beta}, \hat{\lambda})^{\prime} \stackrel{a}{=}\left\{(\alpha, \beta, \lambda)^{\prime} ; \mathrm{I}^{-1}(\psi)\right\}\right)
\end{aligned}
$$

onde, $\mathrm{I}_{0}^{-1}$ é a inversa da matriz de informação observada $\left(\right.$ e $\mathrm{I}^{-1}(\psi)$ é a inversa da matriz de informação de Fisher ).

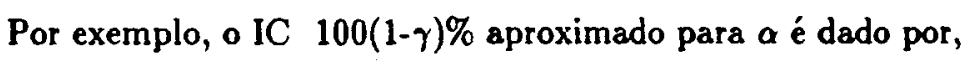

$$
\mathrm{IC}(\alpha)=\left[\hat{\alpha}-\mathrm{Z}_{\gamma / 2} \sqrt{\hat{\mathrm{V}}(\hat{\alpha})}, \hat{\alpha}+\mathrm{Z}_{\gamma / 2} \sqrt{\hat{\mathrm{V}}(\alpha)}\right]
$$

De forma análoga encontramos os intervalos de confiança para $\beta, \lambda$ e $\lambda^{-1}$. O procedimento é similar usando a matriz de informação de Fisher (ver A3.1, Apêndice A3). 


\section{CAPÍTULO 4}

UMA ANÁLISE BAYESIANA PARA O MODELO DE REGRESSĀO LINEAR INVERSO

\subsection{UMA ANÁLISE BAYESIANA ASSUMINDO $\alpha, \beta$ e $\lambda$ DESCONHECIDOS.}

\subsubsection{FUNÇÃO DE VEROSSIMILHANÇA PARA $\alpha, \beta$ e $\lambda$.}

Da equação (3.2) e com a definição das estatísticas básicas dadas em (3.7), a função de verossimilhança para os parâmetros $\alpha, \beta$ e $\lambda$ pode ser escrita por,

$$
L(\alpha, \beta, \lambda) \propto \lambda^{n / 2} \exp \left\{-\frac{\lambda}{2} A(\alpha, \beta)\right\}
$$

onde $\alpha>0, \beta>0$ e $\lambda>0$, e $A(\alpha, \beta)=n \alpha^{2} V_{0}+2 n \alpha \beta V_{1}+n \beta^{2} V_{2}-2 n \alpha-2 n \beta \bar{X}+n R$

\subsubsection{DENSIDADE A PRIORJ PARA $\alpha, \beta \in \lambda$.}

Uma densidade a priori não-informativa para os parâmetros pode ser obtida usando a regra de Jeffreys (ver por exemplo, Box e Tiao, 1973) dada a partir de,

$$
\pi(\alpha, \beta, \lambda)=\pi(\alpha, \beta / \lambda) \pi_{0}(\lambda)
$$

isto é,

$$
\pi(\alpha, \beta, \lambda) \propto\{\operatorname{det} I(\alpha, \beta / \lambda)\}^{1 / 2} \pi_{0}(\lambda)
$$

onde I $(\alpha, \beta / \lambda)$ é a matriz de informação de Fisher dado $\lambda$.

De (3.33) temos, 
1.1 Uma análise bayesiana assumindo $\alpha, \beta$ e $\lambda$ conhecidos - pág. 19 $I(\alpha, \beta / \lambda)=\left[\begin{array}{ll}n \lambda W_{0}(\alpha, \beta) & n \lambda W_{1}(\alpha, \beta) \\ n \lambda W_{1}(\alpha, \beta) & n \lambda W_{2}(\alpha, \beta)\end{array}\right]$

$\operatorname{com} W_{l}(\alpha, \beta)=n^{-1} \sum_{i=1}^{k} \sum_{j=1}^{n_{i}} x_{i}^{l}\left(\alpha+\beta x_{i}\right)^{-1}=n^{-1} \sum_{i=1}^{k} n_{i} x_{i}^{l}\left(\alpha+\beta x_{i}\right)^{-1}, l=0,1,2$, conforme vimos na seçāo 3.4 .

Assim,

$$
\operatorname{det} I(\alpha, \beta / \lambda)=\mathrm{n}^{2} \lambda^{2}\left\{\mathrm{~W}_{0}(\alpha, \beta) \mathrm{W}_{2}(\alpha, \beta)-\mathrm{W}_{1}^{2}(\alpha, \beta)\right\}
$$

Para obtermos a densidade a priori, substituindo (4.4) em (4.2) temos,

$$
\pi(\alpha, \beta, \lambda) \propto n \lambda \sqrt{W_{0}(\alpha, \beta) W_{2}(\alpha, \beta)-W_{1}^{2}(\alpha, \beta)} \pi_{0}(\lambda)
$$

onde $\alpha>0, \beta>0$ e $\lambda>0$.

Assumindo $\pi_{0}(\lambda) \propto \lambda^{-1}$, com $\lambda>0$, encontramos uma Densidade a priori nāoinformativa para $\alpha, \beta$ e $\lambda$ dada por,

$$
\pi(\alpha, \beta, \lambda) \propto\left\{\mathrm{W}_{0}(\alpha, \beta) \mathrm{W}_{2}(\alpha, \beta)-\mathrm{W}_{1}^{2}(\alpha, \beta)\right\}^{1 / 2}
$$

onde $\alpha>0, \beta>0, \lambda>0, \mathrm{e} \mathrm{W}_{l}(\alpha, \beta), l=0,1,2$ é definida em (4.3).

\subsubsection{DENSIDADE A POSTERIOR CONJUNTA PARA $\alpha, \beta$ e $\lambda$.}

Considerando a função de verossimilhança (4.1) e a densidade a priori não-informativa (4.6), a densidade a posteriori conjunta para $\alpha, \beta$ e $\lambda$ é dada por,

$$
\pi(\alpha, \beta, \lambda / \text { dados }) \propto \pi(\alpha, \beta, \lambda) \mathrm{L}(\alpha, \beta, \lambda)
$$

isto é,

$$
\pi(\alpha, \beta, \lambda / \text { dados }) \propto\left\{\mathrm{W}_{0}(\alpha, \beta) \mathrm{W}_{2}(\alpha, \beta)-\mathrm{W}_{1}^{2}(\alpha, \beta)\right\}^{1 / 2} \lambda^{n / 2} \exp \left\{-\frac{\lambda}{2} \mathrm{~A}(\alpha, \beta)\right\}
$$

onde $\alpha>0, \beta>0, \lambda>0$, e $W_{l}(\alpha, \beta), l=0,1,2$ é dada em (4.3) e $A(\alpha, \beta)$ é dada em (4.1). 
1.1 Ume análise bayesiana assumindo $\alpha, \beta$ e $\lambda$ conhecidos - pág. 11 Integrando (4.7) em relação a $\lambda$, obtemos a densidade a posteriori conjunta marginal para $\alpha$ e $\beta$, dada por,

$$
\pi(\alpha, \beta / \text { dados }) \propto\left\{W_{0}(\alpha, \beta) W_{2}(\alpha, \beta)-W_{1}^{2}(\alpha, \beta)\right\}^{1 / 2} \int_{0}^{\infty} \lambda^{n / 2} \exp \left\{-\frac{\lambda}{2} A(\alpha, \beta)\right\} d \lambda
$$

Para resolver (4.8) usamos alguns resultados de integrais conhecidas (ver, Box e Tiao, 1973, pag. 144), (ver (2.39)); e obtemos a Densidade a posteriori conjunta marginal para a $\boldsymbol{\beta}$ dada por,

$$
\pi(\alpha, \beta / \text { dados }) \propto\left\{W_{0}(\alpha, \beta) W_{2}(\alpha, \beta)-W_{1}^{2}(\alpha, \beta)\right\}^{1 / 2} A(\alpha, \beta)-(n / 2+1)
$$

onde $\alpha>0$ e $\beta>0$, com $A(\alpha, \beta)$ definidos em (4.1) e $W_{l}(\alpha, \beta)$ definidos em (4.3).

\subsubsection{DENSIDADE A POSTERIORI MARGINAIS PARA $\alpha, \beta$ e $\lambda$.}

Integrando (4.9) em relação a $\beta$, obtemos a densidade a posteriori marginal para $\alpha$ dada por,

$$
\pi(\alpha / \text { dados }) \propto \int_{0}^{\infty}\left\{\mathrm{W}_{0}(\alpha, \beta) \mathrm{W}_{2}(\alpha, \beta)-\mathrm{W}_{\mathrm{I}}^{2}(\alpha, \beta)\right\}^{1 / 2} \mathrm{~A}(\alpha, \beta)^{-(n / 2+1)} \mathrm{d} \beta
$$

Essa integral pode ser resolvida utilizando o método de Laplace (ver Apêndice A1), para isso reescrevendo (4.10) adequadamente temos,

$$
\pi(\alpha / \text { dados }) \propto \int_{0}^{\infty}\left\{\mathrm{W}_{0}(\alpha, \beta) \mathrm{W}_{2}(\alpha, \beta)-\mathrm{W}_{1}^{2}(\alpha, \beta)\right\}^{1 / 2} \mathrm{~A}(\alpha, \beta)^{-1} \exp \left\{-\mathrm{n}\left(\frac{\log \mathrm{A}(\alpha, \beta)}{2}\right)\right\} \mathrm{d} \beta
$$

e definimos,

$$
\begin{aligned}
& f_{\alpha}(\beta)=\left\{W_{0}(\alpha, \beta) W_{2}(\alpha, \beta)-W_{1}^{2}(\alpha, \beta)\right\}^{1 / 2} A(\alpha, \beta)^{-1}, \text { e } \\
& h_{a}(\beta)=\frac{\log A(\alpha, \beta)}{2} .
\end{aligned}
$$

Daí, obtemos a Densidade a posteriori marginal para $\alpha$ aproximada pelo Método de Laplace dada por, $\pi(\alpha /$ dados $) \propto\left\{\mathrm{W}_{0}(\alpha, \hat{\beta}) \mathrm{W}_{2}(\alpha, \hat{\beta})-\mathrm{W}_{1}^{2}(\alpha, \hat{\beta})\right\}^{1 / 2}\left[\alpha^{2} \mathrm{D}-2 \alpha\left(\mathrm{V}_{2}-\overline{\mathrm{X}} \mathrm{V}_{1}\right)-\overline{\mathrm{X}}^{2}+\mathrm{RV} \mathrm{V}_{2}\right]^{-(n+1) / 2}$

onde $\alpha>0$, e $W_{l}(\alpha, \hat{\beta}), \iota=0,1,2(4.3) \operatorname{com} \dot{\beta}=\frac{\bar{X}-\alpha V_{1}}{V_{2}}$ 
4.1 Uma análise bayesiana assumindo $\alpha, \beta$ e $\lambda$ conhecidos - pág. 45 Analogamente, integrando (4.9) em relação a o obtemos a Densidade a posteriori marginal para $\beta$ aproximada pelo Método de Laplace dada por,

$$
\pi(\beta / \text { dados }) \propto\left\{W_{0}(\hat{\alpha}, \beta) W_{2}(\hat{\alpha}, \beta)-W_{1}^{2}(\hat{\alpha}, \beta)\right\}^{1 / 2}\left[\beta^{2} D+2 \beta\left(V_{1}-\bar{X} V_{0}\right)+R V_{0}-1\right]^{-(n+1) / 2}
$$

onde $\alpha>0, \mathrm{e} \mathrm{W}_{l}(\hat{\alpha}, \beta), \iota=0,1,2 \mathrm{em}(4.3) \operatorname{com} \hat{\alpha}=\frac{1-\beta \mathrm{V}_{1}}{\mathrm{~V}_{0}}$

Integrando (4.7) em relação a $\alpha$ e $\beta$, obtemos a densidade a posteriori marginal para $\lambda$, dada por,

$$
\pi(\lambda / \text { dados }) \propto \int_{0}^{\infty} \int_{0}^{\infty}\left\{W_{0}(\alpha, \beta) W_{2}(\alpha, \beta)-W_{1}^{2}(\alpha, \beta)\right\}^{1 / 2} \lambda^{n / 2} \exp \left\{-\frac{\lambda}{2} A(\alpha, \beta)\right\} \operatorname{dad} \beta
$$

Reescrevendo a integral em (4.14) temos,

$$
\begin{aligned}
& \pi(\lambda / \text { dados }) \propto \lambda^{n / 2} \int_{0}^{\infty} \int_{0}^{\infty}\left\{\mathrm{W}_{0}(\alpha, \beta) \mathrm{W}_{2}(\alpha, \beta)-\mathrm{W}_{1}^{2}(\alpha, \beta)\right\}^{1 / 2} \times \\
& \times \exp \left\{-\mathrm{n} \frac{\lambda}{2}\left(\alpha^{2} \mathrm{~V}_{0}+2 \alpha \beta \mathrm{V}_{1}+\beta^{2} \mathrm{~V}_{2}-2 \alpha-2 \beta \overline{\mathrm{X}}+\mathrm{R}\right)\right\} \mathrm{d} \alpha \mathrm{d} \beta
\end{aligned}
$$

Usando o método de Laplace no caso multiparamétrico (ver Apéndice A1) para aproximar a integral (4.15), com,

$$
\begin{aligned}
& \mathrm{f}(\alpha, \beta)=\left\{\mathrm{w}_{0}(\alpha, \beta) \mathrm{W}_{2}(\alpha, \beta)-\mathrm{W}_{1}^{2}(\alpha, \beta)\right\}^{1 / 2} \\
& \mathrm{~h}(\alpha, \beta)=\frac{\lambda}{2}\left(\alpha^{2} \mathrm{~V}_{0}+2 \alpha \beta \mathrm{V}_{1}+\beta^{2} \mathrm{~V}_{2}-2 \alpha-2 \beta \overline{\mathrm{X}}+\mathrm{R}\right),
\end{aligned}
$$

obtemos a Densidade a posteriori marginal para $\lambda$ aproximada pelo Método de Laplace dada por,

$$
\pi(\lambda / \text { dados }) \propto \lambda^{n / 2-1} \exp \left\{-\frac{n \lambda}{2}\left[R-\frac{\left(V_{2}-2 \bar{X} V_{1}+\bar{X}^{2} V_{0}\right)}{D}\right]\right\}
$$

onde $\lambda>0$. 
1.1 Uma análise bayesiane essumindo $\alpha, \beta$ e $\lambda$ conhecidos - pág. 16 4.1.5 DENSIDADE A POSTERJORI MARGINAL PARA O TEMPO MÉDIO DE FALBA $\theta_{1}$ SOB 0 NIVEL USUAL DE ESTRESSE $X_{1}$.

Usualmente, as industrias tem interesse em fazer inferèncias a respeito de $\theta_{1}=\left(\alpha+\beta x_{1}\right)^{-1}$, onde $x_{1}$ é o nivel usual de estresse. Considere a seguinte transformação de variáveis,

$$
\begin{aligned}
& \theta_{1}=\left(\alpha+\beta \mathrm{x}_{1}\right)^{-1} \\
& \alpha=\alpha
\end{aligned}
$$

de (4.17) obtemos a seguinte relação, $\beta=\frac{1}{\theta_{1} x_{1}}-\frac{\alpha}{x_{1}}=\beta\left(\theta_{1}\right)$.

Para encontrar $\pi\left(\theta_{1} /\right.$ dados $)$ determinamos $\pi\left(\alpha, \theta_{1} /\right.$ dados $)$ pois conhecemos $\pi(\alpha, \beta /$ dados $)$ dada em (4.9) (usando transformação de variáveis) e depois integramos em relação a $\alpha$ encontrando a densidade a posteriori marginal de $\theta_{1}$.

De (4.9) temos,

$$
\pi\left(\alpha, \theta_{1} / \text { dados }\right) \propto \pi\left(\alpha, \beta\left(\theta_{1}\right) / \text { dados }\right) \times|\mathrm{J}|
$$

com,

$$
|\mathrm{J}|=\left|\begin{array}{cc}
\frac{\partial \alpha}{\partial \alpha} & \frac{\partial \alpha}{\partial \theta_{1}} \\
\frac{\partial \beta}{\partial \alpha} & \frac{\partial \beta}{\partial \theta_{1}}
\end{array}\right|=\left|\begin{array}{cc}
1 & 0 \\
-\frac{1}{\mathrm{x}_{1}} & -\frac{1}{\mathrm{x}_{1} \theta_{1}^{2}}
\end{array}\right|=\frac{1}{\mathrm{x}_{1} \theta_{1}^{2}} .
$$

Assim substituindo estes valores em (4.18) temos,

$$
\begin{gathered}
\pi\left(\alpha, \theta_{1} / \text { dados }\right) \propto\left\{\mathrm{W}_{0}\left(\alpha, \beta\left(\theta_{1}\right)\right) \mathrm{W}_{2}\left(\alpha, \beta\left(\theta_{1}\right)\right)-\mathrm{W}_{1}^{2}\left(\alpha, \beta\left(\theta_{1}\right)\right)\right\}^{1 / 2} \times \\
\times\left\{n \alpha^{2} \mathrm{~V}_{0}+2 \mathrm{n} \alpha\left(\frac{1}{\theta_{1} \mathrm{x}_{1}}-\frac{\alpha}{\mathrm{x}_{1}}\right) \mathrm{V}_{1}+\mathrm{n}\left(\frac{1}{\theta_{1} \mathrm{x}_{1}}-\frac{\alpha}{\mathrm{x}_{1}}\right)^{2} \mathrm{~V}_{2}-2 \mathrm{n} \alpha-2 \mathrm{n}\left(\frac{1}{\theta_{1} \mathrm{x}_{1}}-\frac{a}{\mathrm{x}_{1}}\right) \overline{\mathrm{X}}+\mathrm{nR}\right\}^{-(n / 2+1)} \times \frac{1}{\mathrm{x}_{1} \theta_{1}^{2}}
\end{gathered}
$$

Observar que, 


$$
\begin{aligned}
& \left\{n \alpha^{2} V_{0}+2 n \alpha\left(\frac{1}{\theta_{1} x_{1}}-\frac{\alpha}{x_{1}}\right) V_{1}+n\left(\frac{1}{\theta_{1} x_{1}}-\frac{\alpha}{x_{1}}\right)^{2} V_{2}-2 n \alpha-2 n\left(\frac{1}{\theta_{1} x_{1}}-\frac{\alpha}{x_{1}}\right) \bar{X}+n R\right\}= \\
& =n \alpha^{2} V_{0}+\frac{2 n \alpha V_{1}}{\theta_{1} x_{1}}-\frac{2 n \alpha^{2} V_{1}}{x_{1}}+\frac{n V_{2}}{\theta_{1}^{2} x_{1}^{2}}-\frac{2 n \alpha V_{2}}{\theta_{1} x_{1}^{2}}+\frac{n \alpha^{2} V_{2}}{x_{1}^{2}}-2 n \alpha-\frac{2 n \bar{X}}{\theta_{1} x_{1}}+\frac{2 n \alpha \bar{X}}{X_{1}}+n R
\end{aligned}
$$

Podemos definir as seguintes relações,

$$
\begin{aligned}
& a_{1} \alpha^{2}=\left(n V_{0}-\frac{2 n V_{1}}{x_{1}}+\frac{n V_{2}}{x_{1}^{2}}\right) \alpha^{2}, \text { isto é, } a_{1}=n V_{0}-\frac{2 n V_{1}}{x_{1}}+\frac{n V_{2}}{x_{1}^{2}} ; \\
& a_{2}\left(\theta_{1}\right) \alpha=\left(\frac{2 n V_{1}}{\theta_{1} x_{1}}-\frac{2 n V_{2}}{\theta_{1} x_{1}^{2}}-2 n+\frac{2 n \bar{X}}{x_{1}}\right) \alpha, \text { isto é, } a_{2}\left(\theta_{1}\right)=\frac{2 n V_{1}}{\theta_{1} x_{1}}-\frac{2 n V_{2}}{\theta_{1} x_{1}^{2}}-2 n+\frac{2 n \bar{X}}{x_{1}} ; e \\
& a_{3}\left(\theta_{1}\right)=\frac{n V_{2}}{\theta_{1}^{2} x_{1}^{2}}-\frac{2 n \bar{X}}{\theta_{1} x_{1}}+n R .
\end{aligned}
$$

Também, $W_{l}\left(\alpha, \theta_{1}\right)=n^{-1}\left\{n_{1} x_{1}^{l} \theta_{1}+\sum_{i=2}^{k} n_{i} x_{i}^{l}\left[\alpha\left(1-\frac{x_{i}}{x_{1}}\right)+\frac{x_{i}}{\theta_{1} x_{1}}\right]^{-1}\right\}, \iota=0,1,2$.

Substituindo estes valores em (4.19) obtemos a Densidade a posteriori conjunts para a e $\theta_{1}$ dada por,

$$
\left.\pi\left(\alpha, \theta_{1} / \text { dados }\right) \propto \frac{1}{\theta_{1}^{2}} W_{0}\left(\alpha, \theta_{1}\right) W_{2}\left(\alpha, \theta_{1}\right)-W_{1}^{2}\left(\alpha, \theta_{1}\right)\right\}^{1 / 2}\left\{a_{1} \alpha^{2}+a_{2}\left(\theta_{1}\right) \alpha+a_{3}\left(\theta_{1}\right)\right\}^{-(n / 2+1)}
$$

onde $\alpha>0$ e $\theta_{1}>0$.

De (4.20) podemos integrar em relaçâo a $\alpha$ e encontrar a densidade a posteriori marginal para $\theta_{1}$ dada por,

$$
\begin{aligned}
& \pi\left(\theta_{1} / \text { dados }\right) \propto \\
& \propto \frac{1}{\theta_{1}^{2}} \int_{0}^{\infty}\left\{\mathrm{W}_{0}\left(\alpha, \theta_{1}\right) \mathrm{W}_{2}\left(\alpha, \theta_{1}\right)-\mathrm{W}_{1}^{2}\left(\alpha, \theta_{1}\right)\right\}^{1 / 2}\left\{\mathrm{a}_{1} \alpha^{2}+\mathrm{a}_{2}\left(\theta_{1}\right) \alpha+\mathrm{a}_{3}\left(\theta_{1}\right)\right\}^{-(n / 2+1)} \mathrm{d} \alpha
\end{aligned}
$$

Reescrevendo a integral em (4.21) temos,

$$
\begin{aligned}
& \pi\left(\theta_{1} / \text { dados }\right) \propto \\
& \propto \frac{1}{\theta_{1}^{2}} \int_{0}^{\infty} \frac{\left\{W_{0}\left(\alpha, \theta_{1}\right) W_{2}\left(\alpha, \theta_{1}\right)-W_{1}^{2}\left(\alpha, \theta_{1}\right)\right\}^{1 / 2}}{\left\{a_{1} \alpha^{2}+a_{2}\left(\theta_{1}\right) \alpha+a_{3}\left(\theta_{1}\right)\right\}} \exp \left\{-n \frac{1}{2} \log \left\{a_{1} \alpha^{2}+a_{2}\left(\theta_{1}\right) \alpha+a_{3}\left(\theta_{1}\right)\right\}\right\} d \alpha
\end{aligned}
$$


4.2 Uma análise bayesiana assumindo $\lambda$ conhecido - pág. 18 Usando o metódo de Laplace (ver Apéndice A1) para aproximar integral em (4.21) com,

$$
\begin{aligned}
& f_{\theta_{1}}(\alpha)=\frac{\left\{w_{0}\left(\alpha, \theta_{1}\right) w_{2}\left(\alpha, \theta_{1}\right)-w_{1}^{2}\left(\alpha, \theta_{1}\right)\right\}^{1 / 2}}{\left\{a_{1} \alpha^{2}+a_{2}\left(\theta_{1}\right) \alpha+a_{3}\left(\theta_{1}\right)\right\}}, \\
& b_{\theta_{1}}(\alpha)=\frac{1}{2} \log \left\{a_{1} \alpha^{2}+a_{2}\left(\theta_{1}\right) \alpha+a_{3}\left(\theta_{1}\right)\right\}
\end{aligned}
$$

obtemos a Densidade a posteriori marginal para $\theta_{1}$ aproximada pelo Método de Laplace dada por,

$$
\pi\left(\theta_{1} / \text { dados }\right) \propto \theta_{1}^{-2}\left\{W_{0}\left(\hat{\alpha}, \theta_{1}\right) W_{2}\left(\hat{\alpha}, \theta_{1}\right)-W_{1}^{2}\left(\hat{\alpha}, \theta_{1}\right)\right\}^{1 / 2}\left\{4 a_{1} a_{3}\left(\theta_{1}\right)-a_{2}^{2}\left(\theta_{1}\right)\right\}^{-(n+1) / 2}
$$

onde $\theta_{1}>0$.

\subsection{UMA ANÁLISE BAYESIANA ASSUMINDO $\lambda$ CONBECIDO.}

A função de verossimilhança para $\alpha$ e $\beta \operatorname{com} \lambda$ conhecido de (4.1) é dada por,

$$
L(\alpha, \beta) \propto \exp \left\{-\frac{\lambda}{2} \mathrm{~A}(\alpha, \beta)\right\}
$$

onde $\alpha>0, \beta>0$, e $\mathrm{A}(\alpha, \beta)$ é dada em (4.1).

Considere uma priori não-informativa de Jeffreys para $\alpha$ e $\beta$ de (4.6) dada por,

$$
\pi(\alpha, \beta) \propto\left\{\mathrm{W}_{0}(\alpha, \beta) \mathrm{W}_{2}(\alpha, \beta)-\mathrm{W}_{1}^{2}(\alpha, \beta)\right\}^{1 / 2}
$$

onde $\alpha>0, \beta>0$, e $W_{l}(\alpha, \beta), l=0,1,2$ é dada em (4.3).

\subsubsection{DENSIDADE A POSTERIORI CONJUNTA PARA $\alpha$ e $\beta$.}

Considerando a funçâo de verossimilhança (4.24) e a densidade a priori nāo-informativa de Jeffreys (4.25) para $\alpha$ e $\beta$, a densidade a posteriori conjunta para $\alpha$ e $\beta$ é dada por,

$$
\pi(\alpha, \beta / \text { dados }) \propto \pi(\alpha, \beta) \mathrm{L}(\alpha, \beta)
$$


Portanto,

$$
\pi(\alpha, \beta / \text { dados }) \propto\left\{W_{0}(\alpha, \beta) W_{2}(\alpha, \beta)-W_{1}^{2}(\alpha, \beta)\right\}^{1 / 2} \exp \left\{-\frac{\lambda}{2} A(\alpha, \beta)\right\}
$$

onde $\alpha>0, \beta>0$ e $\lambda$ é conhecido, e $W_{l}(\alpha, \beta), l=0,1,2$ é dada em (4.3) e $A(\alpha, \beta)$ é dada em (4.1).

\subsubsection{DENSIDADE A POSTERIORI MARGINAL PARA $\alpha \in \beta$.}

Integrando (4.26) em relação a $\beta$, obtemos a densidade a posteriori marginal para $\alpha$ dada por,

$$
\pi(\alpha / \text { dados }) \propto \int_{0}^{\infty}\left\{W_{0}(\alpha, \beta) W_{2}(\alpha, \beta)-W_{1}^{2}(\alpha, \beta)\right\}^{1 / 2} \exp \left\{-\frac{\lambda}{2} A(\alpha, \beta)\right\} d \beta
$$

Reescrevendo a integral em (4.27), temos,

$\pi(a /$ dados $) \propto$

$\alpha \int_{0}^{\infty}\left\{\mathrm{W}_{0}(\alpha, \beta) \mathrm{W}_{2}(\alpha, \beta)-\mathrm{W}_{1}^{2}(\alpha, \beta)\right\}^{1 / 2} \exp \left\{-\mathrm{n} \frac{\lambda}{2}\left(\alpha^{2} \mathrm{~V}_{0}+2 \alpha \beta \mathrm{V}_{1}+\beta^{2} \mathrm{~V}_{2}-2 \alpha-2 \beta \overline{\mathrm{X}}+\mathrm{R}\right)\right\} \mathrm{d} \beta$

Usando a metódo de Laplace (ver Apêndice A1) com,

$$
\begin{aligned}
& \mathrm{f}_{\alpha}(\beta)=\left\{\mathrm{W}_{0}(\alpha, \beta) \mathrm{W}_{2}(\alpha, \beta)-\mathrm{W}_{1}^{2}(\alpha, \beta)\right\}^{1 / 2} \mathrm{e} \\
& \mathrm{h}_{\alpha}(\beta)=\frac{\lambda}{2}\left(\alpha^{2} \mathrm{~V}_{0}+2 \alpha \beta \mathrm{V}_{1}+\beta^{2} \mathrm{~V}_{2}-2 \alpha-2 \beta \overline{\mathrm{X}}+\mathrm{R}\right),
\end{aligned}
$$

obtemos a Densidade a posteriori marginal para a aproximada pelo Método de Laplace dada por,

$\pi(\alpha /$ dados $) \propto\left\{\mathrm{W}_{0}(\alpha, \hat{\beta}) \mathrm{W}_{2}(\alpha, \dot{\beta})-\mathrm{W}_{1}^{2}(\alpha, \dot{\beta})\right\}^{1 / 2} \exp \left\{-\mathrm{n} \frac{\lambda}{2 \mathrm{~V}_{2}}\left(\alpha^{2} \mathrm{D}-2 \alpha\left(\mathrm{V}_{2}-\overline{\mathrm{X}} \mathrm{V}_{1}\right)+\mathrm{R} \mathrm{V}_{2}-\overline{\mathrm{X}}^{2}\right)\right\}$

onde $\alpha>0, \lambda$ conhecido, e $W_{l}(\alpha, \beta), \imath=0,1,2$ é dada em $(4.3)$ com $\hat{\beta}=\frac{\bar{X}-\alpha V_{1}}{V_{2}}$

Analogamente, integrando (4.26) em relaşão a $\alpha$, obtemos a Densidạde a posteriori marginal para $\beta$ aproximada pelo Método de Laplace dada por, 
$\pi(\beta /$ dados $) \propto\left\{\mathrm{W}_{0}(\hat{\alpha}, \beta) \mathrm{W}_{2}(\dot{\alpha}, \beta)-\mathrm{W}_{1}^{2}(\hat{\alpha}, \beta)\right\}^{1 / 2} \exp \left\{-\mathrm{n} \frac{\lambda}{2 \mathrm{~V}_{0}}\left(\beta^{2} \mathrm{D}+2 \beta\left(\mathrm{V}_{1}-\overline{\mathrm{X}} \mathrm{V}_{0}\right)+\mathrm{R} \mathrm{V}_{0}-1\right)\right\}$

onde $\beta>0, \lambda$ conhecido, e $W_{l}(\hat{\alpha}, \beta), l=0,1,2$ é dada em (4.3) com $\dot{\alpha}=\frac{1-\beta V_{1}}{V_{0}}$.

\subsubsection{DENSIDADE A POSTERIORI MARGINAL PARA O TEMPO MÉDIO DE FALHA $\theta_{1}$ SOB 0} NÍVEL USUAL DE ESTRESSE $x_{1}$ COM $\lambda$ CONHECIDO.

De (4.26) e das transformaçōes (4.17) e da relação encontrada $\beta=\frac{1}{\theta_{1} \mathrm{x}_{1}}-\frac{\alpha}{\mathrm{x}_{1}}=\beta\left(\theta_{1}\right)$, determinamos $\pi\left(\theta_{1} /\right.$ dados $)$. Para isto calculamos $\pi\left(\alpha, \theta_{1} /\right.$ dados) a partir de $\pi$ ( $\alpha, \beta /$ dados) (usando transformaçōes de variáveis) e integramos em relação a $\alpha$, para encontrar a densidade a posteriori marginal para $\theta_{1}$. Analogamente ao desenvolvimento feito na seção 4.1 .5 obtemos a Densidade a posteriori conjunta para $\alpha$ e $\theta_{1}$ dada por,

$\pi\left(\alpha, \theta_{1} /\right.$ dados $) \propto \frac{1}{\theta_{1}^{2}}\left\{W_{0}\left(\alpha, \theta_{1}\right) W_{2}\left(\alpha, \theta_{1}\right)-W_{1}^{2}\left(\alpha, \theta_{1}\right)\right\}^{1 / 2} \exp \left\{-\frac{\lambda}{2}\left[a_{1} \alpha^{2}+a_{2}\left(\theta_{1}\right) \alpha+a_{3}\left(\theta_{1}\right)\right]\right\}$

onde $\alpha>0$ e $\theta_{1}>0$.

A Densidade a posteriori marginal para $\theta_{1}$ aproximada pelo Método de Laplace é dada por, $\pi\left(\theta_{1} /\right.$ dados $) \propto \theta_{1}^{-2}\left\{\mathbf{W}_{0}\left(\hat{\alpha}, \theta_{1}\right) W_{2}\left(\hat{\alpha}, \theta_{1}\right)-W_{1}^{2}\left(\hat{\alpha}, \theta_{1}\right)\right\}^{1 / 2} \exp \left\{-\frac{\lambda}{8 a_{1}}\left[4 a_{1} a_{3}\left(\theta_{1}\right)-a_{2}^{2}\left(\theta_{1}\right)\right]\right\}$

onde $\theta_{1}>0$ e $\lambda$ conhecido. 
4.9 - intervalos de credibilidade para os paràmetros - pág. 51

\subsection{INTERVALOS DE CREDIBILIDADE PARA OS PARĀMETROS.}

Após ter obtido as densidades a posteriori para os parâmetros de interesse, é geralmente possível calcular a probabilidade de que o parâmetro se encontre numa determinada região em particular. Esta probabilidade mede o grau de credibilidade que $O(8)$ parámetro(s) pertença a esta regiâo dado a amostra dos dados e a informação a priori. Frequentemente, é usada a noção de Highest posterior density (HPD) (ver por exemplo, Box e Tiao, 1973) para determinar um Intervalo de Credibilidade (ICR) apropriado.

Construiremos intervalos de credibilidade para os parâmetros $\alpha, \beta, \lambda$ e $\theta_{1}$ em torno das modas a partir das densidades a posteriori obtidas nas seções anteriores.

\section{INTERVALO DE CREDIBILIDADE PARA $\alpha$.}

Uma aproximação normal para a densidade a posteriori para $\alpha$ é dada por,

$$
\alpha \stackrel{\mathrm{a}}{\sim}\left[\hat{\alpha},\left(-\left.\frac{\partial^{2} \log \pi(\alpha / \text { dados })}{\partial \alpha^{2}}\right|_{\alpha=\hat{\alpha}}\right)^{-1}\right]
$$

onde $\pi$ ( $\alpha$ / dados) é dada em (4.12), e à é sua moda.

Logo um ICR $100(1-\gamma) \%$ aproximado para $\alpha$ é dado por,

$\hat{\alpha}-\mathrm{Z}_{\gamma / 2}\left(-\left.\frac{\partial^{2} \log \pi(\alpha / \text { dados })}{\partial \alpha^{2}}\right|_{\alpha=\hat{\alpha}}\right)^{-1 / 2}<\alpha<\hat{\alpha}+\mathrm{Z}_{\gamma / 2}\left(-\left.\frac{\partial^{2} \log \pi(\alpha / \text { dados })}{\partial \alpha^{2}}\right|_{\alpha=\hat{\alpha}}\right)^{-1 / 2}$

onde $Z_{\gamma / 2}$ é o percentil de uma distribuição normal padronizada (ver Apêndice A3), e

$$
\frac{\partial^{2} \log \pi(\alpha / \text { dados })}{\partial \alpha^{2}}=\frac{1}{2}\left\{\frac{A^{* \prime \prime}(\alpha) A^{*}(\alpha)-\left(A^{* \prime}(\alpha)\right)^{2}}{A^{*}(\alpha)^{2}}\right\}-\left(\frac{n+1}{2}\right)\left\{\frac{B^{* \prime \prime}(\alpha) B^{*}(\alpha)-\left(B^{* \prime \prime}(\alpha)\right)^{2}}{B^{*}(\alpha)^{2}}\right\}
$$

onde,

$$
\begin{aligned}
& A^{*}(\alpha)=\left\{W_{0}(\alpha, \hat{\beta}) W_{2}(\alpha, \hat{\beta})-W_{1}^{2}(\alpha, \hat{\beta})\right\} \\
& A^{* \prime}(\alpha)=W_{0}^{\prime}(\alpha, \hat{\beta}) W_{2}(\alpha, \hat{\beta})+W_{0}(\alpha, \hat{\beta}) W_{2}^{\prime}(\alpha, \hat{\beta})-2 W_{1}(\alpha, \hat{\beta}) W_{1}^{\prime}(\alpha, \hat{\beta}),
\end{aligned}
$$




$$
\begin{aligned}
& \mathrm{A}^{* \prime \prime}(\alpha)=\mathrm{W}_{0}^{\prime \prime}(\alpha, \dot{\beta}) \mathrm{W}_{2}(\alpha, \dot{\beta})+2 \mathrm{~W}_{0}^{\prime}(\alpha, \dot{\beta}) \mathrm{W}_{2}^{\prime}(\alpha, \hat{\beta})+\mathrm{W}_{0}(\alpha, \dot{\beta}) \mathrm{W}_{2}^{\prime \prime}(\alpha, \dot{\beta})- \\
& -2\left[\mathrm{~W}_{1}^{\prime}(\alpha, \dot{\beta})\right]^{2}-2 \mathrm{~W}_{1}(\alpha, \hat{\beta}) \mathrm{W}_{1}^{\prime \prime}(\alpha, \dot{\beta}) \\
& \mathrm{B}^{*}(\alpha)=\left\{\mathrm{D} \alpha^{2}-2\left(\mathrm{~V}_{2}-\overline{\mathrm{X}} \mathrm{V}_{1}\right) \alpha+\mathrm{RV}_{2}-\overline{\mathrm{X}}^{2}\right\} \\
& \mathrm{B}^{* \prime}(\alpha)=2 \mathrm{D} \alpha-2\left(\mathrm{~V}_{2}-\overline{\mathrm{X}} \mathrm{V}_{1}\right) \\
& \mathrm{B}^{* \prime \prime}(\alpha)=2 \mathrm{D}
\end{aligned}
$$

$\operatorname{Com} \hat{\beta}=\frac{\bar{X}-\alpha V_{1}}{V_{2}}, \operatorname{temos} W_{l}(\alpha, \hat{\beta})=n^{-1} \sum_{i=1}^{k} n_{i} x_{i}^{l} \frac{V_{2}}{\left[\alpha V_{2}+\bar{X} x_{i}-\alpha V_{1} x_{i}\right]}$,

$$
\begin{aligned}
& W_{l}^{\prime}(\alpha, \hat{\beta})=-n^{-1} \sum_{i=1}^{k} n_{i} x_{i}^{l} * \frac{V_{2}\left(V_{2}-V_{1} x_{j}\right)}{\left[\left(V_{2}-V_{1} x_{i}\right) \alpha+\bar{X} x_{i}\right]^{2}}, e \\
& W_{l}^{\prime \prime}(\alpha, \hat{\beta})=2 n^{-1} \sum_{i=1}^{k} n_{i} x_{i}^{l} * \frac{V_{2}\left(V_{2}-V_{1} x_{i}\right)^{2}}{\left[\left(V_{2}-V_{1} x_{i}\right) \alpha+\bar{X} x_{i}\right]^{3}}
\end{aligned}
$$

\section{INTERVALO DE CREDIBILIDADE PARA $\beta$.}

Uma aproximação normal para a densidade a posteriori para $\beta$ é dado por,

$$
\beta \stackrel{a}{\sim} \mathrm{N}\left[\hat{\beta},\left(-\left.\frac{\partial^{2} \log \pi(\beta / \text { dados })}{\partial \beta^{2}}\right|_{\beta=\hat{\beta}}\right)^{-1}\right]
$$

onde $\pi$ ( $\beta$ / dados) é dado em (4.13), e $\hat{\beta}$ é sua moda.

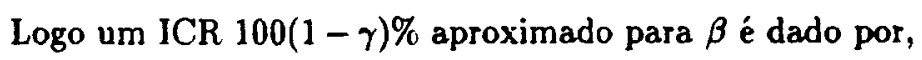

$$
\dot{\beta}-Z_{\gamma / 2}\left(-\left.\frac{\partial^{2} \log \pi(\beta / \text { dados })}{\partial \beta^{2}}\right|_{\beta=\hat{\beta}}\right)^{-1 / 2}<\beta<\dot{\beta}+Z_{\gamma / 2}\left(-\left.\frac{\partial^{2} \log \pi(\beta / \text { dados })}{\partial \beta^{2}}\right|_{\beta=\hat{\beta}}\right)^{-1 / 2}
$$

onde $Z_{\gamma / 2}$ é o percentil da distribuição normal padronizada, e 


$$
\frac{\partial^{2} \log \pi(\beta / \text { dados })}{\partial \beta^{2}}=\frac{1}{2}\left\{\frac{A^{* \prime \prime}(\beta) A^{*}(\beta)-\left(A^{* \prime}(\beta)\right)^{2}}{A^{*}(\beta)^{2}}\right\}-\left(\frac{n+1}{2}\right)\left\{\frac{B^{* \prime \prime}(\beta) B^{*}(\beta)-\left(B^{* \prime}(\beta)\right)^{2}}{B^{*}(\beta)^{2}}\right\}
$$

onde,

$$
\begin{aligned}
& \mathrm{A}^{*}(\beta)=\left\{\mathrm{W}_{0}(\hat{\alpha}, \beta) \mathrm{W}_{2}(\hat{\alpha}, \beta)-\mathrm{W}_{1}^{2}(\hat{\alpha}, \beta)\right\}, \\
& \mathrm{A}^{* \prime}(\beta)=\mathrm{W}_{0}^{\prime}(\hat{\alpha}, \beta) \mathrm{W}_{2}(\hat{\alpha}, \beta)+\mathrm{W}_{0}(\hat{\alpha}, \beta) \mathrm{W}_{2}^{\prime}(\hat{\alpha}, \beta)-2 \mathrm{~W}_{1}(\hat{\alpha}, \beta) \mathrm{W}_{1}^{\prime}(\hat{\alpha}, \beta), \\
& \mathrm{A}^{\prime \prime \prime}(\beta)=\mathrm{W}_{0}^{\prime \prime}(\hat{\alpha}, \beta) \mathrm{W}_{2}(\hat{\alpha}, \beta)+2 \mathrm{~W}_{0}^{\prime}(\hat{\alpha}, \beta) \mathrm{W}_{2}^{\prime}(\hat{\alpha}, \beta)+\mathrm{W}_{0}(\hat{\alpha}, \beta) \mathrm{W}_{2}^{\prime \prime}(\hat{\alpha}, \beta)- \\
& -2\left[\mathrm{~W}_{1}^{\prime}(\hat{\alpha}, \beta)\right]^{2}-2 \mathrm{~W}_{1}(\hat{\alpha}, \beta) \mathrm{W}_{1}^{\prime \prime}(\hat{\alpha}, \beta), \\
& \mathrm{B}^{*}(\beta)=\left\{\mathrm{D} \beta^{2}+2\left(\mathrm{~V}_{1}-\overline{\mathrm{X}} \mathrm{V}_{0}\right) \beta+\mathrm{RV}_{0}-1\right\}, \\
& \mathrm{B}^{* \prime}(\beta)=2 \mathrm{D} \beta+2\left(\mathrm{~V}_{1}-\overline{\mathrm{X}} \mathrm{V}_{0}\right), \\
& \mathrm{B}^{* \prime \prime}(\beta)=2 \mathrm{D} .
\end{aligned}
$$

$\operatorname{Com} \dot{\alpha}=\frac{1-\beta V_{1}}{V_{0}}, \operatorname{temos} W_{l}(\alpha, \hat{\beta})=n^{-1} \sum_{i=1}^{k} n_{i} x_{i}^{l} \frac{V_{0}}{\left[\left(V_{0} x_{i}-V_{1}\right) \beta+1\right]}$,

$$
\begin{aligned}
& W_{l}^{\prime}(\hat{\alpha}, \beta)=-n^{-1} \sum_{i=1}^{k} n_{i} x_{i}^{l} * \frac{V_{0}\left(V_{0} x_{i}-V_{1}\right)}{\left[\left(V_{0} x_{i}-V_{1}\right) \beta+1\right]^{2}}, e \\
& W_{l}^{\prime \prime}(\hat{\alpha}, \beta)=2 n^{-1} \sum_{i=1}^{k} n_{i} x_{i}^{l} * \frac{V_{0}\left(V_{0} x_{i}-V_{1}\right)^{2}}{\left[\left(V_{0} x_{i}-V_{1}\right) \beta+1\right]^{3}}
\end{aligned}
$$

\section{INTERVALO DE CREDIBILIDADE PARA $\lambda$.}

Uma aproximação normal para a densidade a posteriori para $\lambda$ é dada por,

$$
\lambda \stackrel{a}{\approx}\left[\dot{\lambda},\left(-\left.\frac{\partial^{2} \log \pi(\lambda / \text { dados })}{\partial \lambda^{2}}\right|_{\lambda=\dot{\lambda}}\right)^{-1}\right]
$$

onde $\pi$ ( $\lambda$ / dados) é dado em (4.16), e $\hat{\lambda}$ é sua moda.

Logo um ICR 100(1- $\gamma) \%$ aproximado para $\lambda$ é dado por, 
$\dot{\lambda}-Z_{\gamma / 2}\left(-\left.\frac{\partial^{2} \log \pi(\lambda / \text { dados })}{\partial \lambda^{2}}\right|_{\lambda=\hat{\lambda}}\right)^{-1 / 2}<\lambda<\dot{\lambda}+Z_{\gamma / 2}\left(-\left.\frac{\partial^{2} \log \pi(\lambda / \text { dados })}{\partial \lambda^{2}}\right|_{\lambda=\hat{\lambda}}\right)^{-1 / 2}$

onde $Z_{\gamma / 2}$ é o percentil da distribuição normal padronizada, e

$$
\frac{\partial^{2} \log \pi(\lambda / \text { dados })}{\partial \lambda^{2}}=-\left(\frac{n}{2}-1\right) \frac{1}{\lambda^{2}}
$$

Portanto,

$\lambda \stackrel{a}{\sim}\left[\dot{\lambda}, \frac{1}{\left(\frac{n}{2}-1\right) \frac{1}{\dot{\lambda}^{2}}}\right]$

\section{INTERVALO DE CREDIBILIDADE PARA $\theta_{1}$.}

Uma aproximação normal para a densidade a posteriori para $\theta_{1}$ é dada por,

$$
\theta_{1} \stackrel{a}{\sim} \mathrm{N}\left[\hat{\theta_{1}},\left(-\left.\frac{\partial^{2} \log \pi\left(\theta_{1} / \text { dados }\right)}{\partial \theta_{1}^{2}}\right|_{\theta_{1}=\hat{\theta}_{1}}\right)^{-1}\right]
$$

onde $\pi\left(\theta_{1} /\right.$ dados $)$ é dado em (4.23), e $\hat{\theta}_{1}$ é sua moda.

Logo um ICR 100(1- $\gamma) \%$ aproximado para $\theta_{1}$ é dado por,

$\dot{\theta_{1}}-\mathrm{Z}_{\gamma / 2}\left(-\left.\frac{\partial^{2} \log \pi\left(\theta_{1} / \text { dados }\right)}{\partial \theta_{1}^{2}}\right|_{\theta_{1}=\hat{\theta}_{1}}\right)^{-1 / 2}<\theta_{1}<\dot{\theta_{1}}+Z_{\gamma / 2}\left(-\left.\frac{\partial^{2} \log \pi\left(\theta_{1} / \text { dados }\right)}{\partial \theta_{1}^{2}}\right|_{\theta_{1}=\hat{\theta_{1}}}\right)^{-1 / 2}$

onde $Z_{\gamma / 2}$ é o percentil de uma distribuição normal padronizada, e

$$
\begin{aligned}
& \frac{\partial^{2} \log \pi\left(\theta_{1} / \text { dados }\right)}{\partial \theta_{1}^{2}}=\frac{2}{\theta_{1}^{2}}+\frac{1}{2}\left\{\frac{\mathrm{A}^{* \prime \prime}\left(\theta_{1}\right) \mathrm{A}^{*}\left(\theta_{1}\right)-\left(\mathrm{A}^{* \prime}\left(\theta_{1}\right)\right)^{2}}{\mathrm{~A}^{*}\left(\theta_{1}\right)^{2}}\right\}- \\
& -\left(\frac{\mathrm{n}+1}{2}\right)\left\{\frac{\mathrm{B}^{* \prime \prime}\left(\theta_{1}\right) \mathrm{B}^{*}\left(\theta_{1}\right)-\left(\mathrm{B}^{* \prime}\left(\theta_{1}\right)\right)^{2}}{\mathrm{~B}^{*}\left(\theta_{1}\right)^{2}}\right\},
\end{aligned}
$$


onde,

$$
\begin{aligned}
& A^{*}\left(\theta_{1}\right)=\left\{W_{0}\left(\dot{\alpha}, \theta_{1}\right) W_{2}\left(\dot{\alpha}, \theta_{1}\right)-W_{1}^{2}\left(\dot{\alpha}, \theta_{1}\right)\right\}, \\
& A^{* \prime}\left(\theta_{1}\right)=W_{0}^{\prime}\left(\dot{\alpha}, \theta_{1}\right) W_{2}\left(\dot{\alpha}, \theta_{1}\right)+W_{0}\left(\hat{\alpha}, \theta_{1}\right) W_{2}^{\prime}\left(\dot{\alpha}, \theta_{1}\right)-2 W_{1}\left(\dot{\alpha}, \theta_{1}\right) W_{1}^{\prime}\left(\hat{\alpha}, \theta_{1}\right), \\
& A^{* \prime \prime}\left(\theta_{1}\right)=W_{0}^{\prime \prime}\left(\hat{\alpha}, \theta_{1}\right) W_{2}\left(\dot{\alpha}, \theta_{1}\right)+2 W_{0}^{\prime}\left(\hat{\alpha}, \theta_{1}\right) W_{2}^{\prime}\left(\hat{\alpha}, \theta_{1}\right)+W_{0}\left(\dot{\alpha}, \theta_{1}\right) W_{2}^{\prime \prime}\left(\hat{\alpha}, \theta_{1}\right)- \\
& -2\left[W_{1}^{\prime}\left(\hat{\alpha}, \theta_{1}\right)\right]^{2}-2 W_{1}\left(\dot{\alpha}, \theta_{1}\right) W_{1}^{\prime \prime}\left(\hat{\alpha}, \theta_{1}\right), \\
& \mathrm{B}^{*}\left(\theta_{1}\right)=\left\{4 a_{1} a_{3}\left(\theta_{1}\right)-a_{2}^{2}\left(\theta_{1}\right)\right\}, \\
& B^{* \prime}\left(\theta_{1}\right)=4\left(a_{1}^{\prime} a_{3}\left(\theta_{1}\right)+a_{1} a_{3}^{\prime}\left(\theta_{1}\right)\right)-2 a_{2}\left(\theta_{1}\right) a_{2}^{\prime}\left(\theta_{1}\right)=4 a_{1} a_{3}^{\prime}\left(\theta_{1}\right)-2 a_{2}\left(\theta_{1}\right) a_{2}^{\prime}\left(\theta_{1}\right), \\
& B^{* \prime \prime}\left(\theta_{1}\right)=4\left(a_{1}^{\prime} a_{3}^{\prime}\left(\theta_{1}\right)+a_{1} a_{3}^{\prime \prime}\left(\theta_{1}\right)\right)-2\left(a_{2}^{\prime}\left(\theta_{1}\right) a_{2}^{\prime}\left(\theta_{1}\right)+a_{2}\left(\theta_{1}\right) a_{2}^{\prime \prime}\left(\theta_{1}\right)\right), \\
& \left.=4 a_{1} a_{3}^{\prime \prime}\left(\theta_{1}\right)-2\left[a_{2}^{\prime}\left(\theta_{1}\right)\right]^{2}-2 a_{2}\left(\theta_{1}\right) a_{2}^{\prime \prime}\left(\theta_{1}\right)\right) .
\end{aligned}
$$

Com $\hat{\alpha}=-\frac{a_{2}\left(\theta_{1}\right)}{2 a_{1}}$, temos $W\left(\hat{\alpha}, \theta_{1}\right)=n^{-1} n_{1} x_{1}^{l} \theta_{1}+n^{-1} \sum_{i=2}^{k} n_{i} x_{i}^{l} \frac{1}{\left[\left(\frac{x_{i}}{2 a_{1} x_{1}}-\frac{1}{2 a_{1}}\right) a_{2}\left(\theta_{1}\right)+\frac{x_{i}}{\theta_{1} x_{1}}\right]}$,

$$
W_{l}^{\prime}\left(\hat{\alpha}, \theta_{1}\right)=n^{-1} n_{1} x_{1}^{\prime} \theta_{1}+n^{-1} \sum_{i=2}^{k} n_{i} x_{i}^{l}\left[\frac{\left.\left(\frac{x_{i}}{2 a_{1} x_{1}}-\frac{1}{2 a_{1}}\right) a_{2}^{\prime}\left(\theta_{1}\right)-\frac{x_{i}}{\theta_{1}^{2} x_{1}}\right]}{\left.\left(\frac{x_{i}}{2 a_{1} x_{1}}-\frac{1}{2 a_{1}}\right) a_{2}\left(\theta_{1}\right)+\frac{x_{i}}{\theta_{1} x_{1}}\right]}, e\right.
$$

$\mathbf{W}_{l}^{\prime \prime}\left(\hat{\alpha}, \theta_{1}\right)=\frac{\partial\left(\mathbf{W}_{l}^{\prime}\left(\hat{\alpha}, \theta_{1}\right)\right)}{\partial \theta_{1}^{2}}=$

$=-n^{-1} \sum_{i=2}^{k} n_{i} x_{i}^{L} \frac{\left[\left(\frac{x_{i}}{2 a_{1} x_{1}}-\frac{1}{2 a_{1}}\right) a_{2}^{\prime \prime}\left(\theta_{1}\right)+\frac{2 x_{i}}{\theta_{1}^{3} x_{1}}\right]\left[\left(\frac{x_{i}}{2 a_{1} x_{1}}-\frac{1}{2 a_{1}}\right) a_{2}\left(\theta_{1}\right)+\frac{x_{i}}{\theta_{1} x_{1}}\right]-\left[\left(\frac{x_{i}}{2 a_{1} x_{1}}-\frac{1}{2 a_{1}}\right) a_{2}^{\prime}\left(\theta_{1}\right)-\frac{x_{i}}{\theta_{1}^{2} x_{1}}\right]^{2}}{\left[\left(\frac{x_{i}}{2 a_{1} x_{1}}-\frac{1}{2 a_{1}}\right) a_{2}\left(\theta_{1}\right)+\frac{x_{i}}{\theta_{1} x_{1}}\right]^{2}}$

Também observar que,

$$
\begin{aligned}
& a_{1}^{\prime}=0 \text {, e } a_{1}^{\prime \prime}=0 ; \\
& a_{2}^{\prime}\left(\theta_{1}\right)=\frac{\partial a_{2}\left(\theta_{1}\right)}{\partial \theta_{1}}=\frac{2 n V_{2}}{\theta_{1}^{2} x_{1}^{2}}-\frac{2 n V_{1}}{\theta_{1}^{2} x_{1}}, \text { e } a_{2}^{\prime \prime}\left(\theta_{1}\right)=\frac{\partial\left(a_{2}^{\prime}\left(\theta_{1}\right)\right)}{\partial \theta_{1}}=\frac{4 n V_{1}}{\theta_{1}^{3} x_{1}}-\frac{4 n V_{2}}{\theta_{1}^{3} x_{1}^{2}} ; \\
& a_{3}^{\prime}\left(\theta_{1}\right)=\frac{2 n \bar{X}}{\theta_{1}^{2} x_{1}}-\frac{2 n V_{2}}{\theta_{1}^{3} x_{1}^{2}}, a_{3}^{\prime \prime}\left(\theta_{1}\right)=\frac{\partial\left(a_{3}^{\prime}\left(\theta_{1}\right)\right)}{\partial \theta_{1}}=\frac{6 n V_{2}}{\theta_{1}^{4} x_{1}^{2}}-\frac{4 n \bar{X}}{\theta_{1}^{3} x_{1}}
\end{aligned}
$$


4.9 - intervalos de credibilidade para os parämetros - pág. 56 INTERVALOS DE CREDIBILIDADE PARA $\alpha, \beta$ e $\theta_{1}$ COM $\lambda$ CONHECIDO.

De (4.29), (4.30) e (4.32) um ICR $100(1-\gamma) \%$ aproximado para $\alpha, \beta$ e $\theta_{1}$ tem a forma análoga ao dado em (4.33), (4.34) e (4.35) respectivamente onde,

$$
\begin{aligned}
\frac{\partial^{2} \log \pi(\alpha / \text { dados })}{\partial \alpha^{2}}= & \frac{1}{2}\left\{\frac{A^{* \prime \prime}(\alpha) A^{*}(\alpha)-\left(A^{* \prime}(\alpha)\right)^{2}}{A^{* \prime}(\alpha)^{2}}\right\}-\frac{n \lambda D}{V_{2}} \\
\frac{\partial^{2} \log \pi(\beta / \text { dados })}{\partial \beta^{2}}= & \frac{1}{2}\left\{\frac{A^{* \prime \prime}(\beta) A^{*}(\beta)-\left(A^{* \prime \prime}(\beta)\right)^{2}}{A^{*}(\beta)^{2}}\right\}-\frac{n \lambda D}{V_{0}} e \\
\frac{\partial^{2} \log \pi\left(\theta_{1} / \text { dados }\right)}{\partial \theta_{1}^{2}}= & \frac{2}{\theta_{1}^{2}}+\frac{1}{2}\left\{\frac{A^{* \prime \prime}\left(\theta_{1}\right) A^{*}\left(\theta_{1}\right)-\left(A^{* \prime}\left(\theta_{1}\right)\right)^{2}}{A^{*}\left(\theta_{1}\right)^{2}}\right\}-\frac{\lambda}{8 a_{1}}\left\{4 a_{1} a_{3}^{\prime \prime}\left(\theta_{1}\right)-\right. \\
& \left.-2\left[a_{2}^{\prime}\left(\theta_{1}\right)\right]^{2}-2 a_{2}\left(\theta_{1}\right) a_{2}^{\prime \prime}\left(\theta_{1}\right)\right\} .
\end{aligned}
$$




\section{CAPÍTULO 5}

\section{DENSIDADES PREDITIVAS}

\subsection{DENSIDADE PREDITIVA PARA UMA OBSERVAÇÃO FUTURA.}

Para encontrar a densidade preditiva para uma observacão futura, assumimos inicialmente os parâmetros $\alpha$ e $\beta$ conhecidos e uma densidade a priori não-informativa para $\lambda$ dada por,

$$
\pi(\lambda) \propto \lambda^{-1}
$$

onde $\lambda>0$.

A função de verossimilhança para $\lambda$ é dada por,

$$
L(\lambda) \propto \lambda^{n / 2} \exp \left\{-\frac{\lambda}{2} A(\alpha, \beta)\right\}
$$

com $\mathrm{A}(\alpha, \beta)=\mathrm{n}\left(\alpha^{2} \mathrm{~V}_{0}+2 \alpha \beta \mathrm{V}_{1}+\beta^{2} \mathrm{~V}_{2}-2 \alpha-2 \beta \overline{\mathrm{X}}+\mathrm{R}\right)$, e $\alpha, \beta$ conhecidos.

A densidade a posteriori para $\lambda$ é dada por,

$$
\pi(\lambda / \text { dados })=c \pi(\lambda) L(\lambda)
$$

onde $\mathrm{c}$ é a constante normalizadora.

Assim, substituindo (5.1) e (5.2) em (5.3) temos,

$$
\pi(\lambda / \text { dados })=c \lambda^{-1} \lambda^{n / 2} \exp \left\{-\frac{\lambda}{2} A(\alpha, \beta)\right\}
$$




$$
=c \lambda^{n / 2-1} \exp \left\{-\frac{\lambda}{2} A(\alpha, \beta)\right\}
$$

onde $\lambda>0$ e $\alpha, \beta$ são conhecidos.

Para determinar o valor de cem (5.4) temos,

$$
c^{-1}=\int_{0}^{\infty} \lambda^{n / 2-1} \exp \left\{-\frac{\lambda}{2} A(\alpha, \beta)\right\} d \lambda
$$

Esta integral (5.5) tem forma conhecida (ver, Box e Tiao, 1973, pag. 144).

Assim,

$$
c^{-1}=\frac{\Gamma\left(\frac{n}{2}\right)}{\left(\frac{A(\alpha, \beta)}{2}\right)^{n / 2}}, \text { isto } \dot{e}, c=\frac{A(\alpha, \beta)^{n / 2}}{2^{n / 2} \Gamma\left(\frac{n}{2}\right)}
$$

Portanto,

$$
\pi(\lambda / \text { dados })=\frac{A(\alpha, \beta)^{n / 2}}{2^{n / 2} \Gamma\left(\frac{n}{2}\right)} \lambda^{n / 2-1} \exp \left\{-\frac{\lambda}{2} A(\alpha, \beta)\right\}
$$

onde $\lambda>0$ e $n=\sum_{i=1}^{k} n_{i}$ é o número total de observações nos $k$ níveis da variável estresse.

A densidade preditiva para uma observação futura $Y_{(n+1) i}$ é dada por,

$$
\begin{aligned}
& f^{i}\left(y_{(n+1) i} / \text { dados }\right)=\int_{0}^{\infty} f^{i}\left(y_{(n+1) i} / \lambda\right) \pi(\lambda / \text { dados }) d \lambda \\
& =E_{\lambda / \operatorname{dados}}\left\{f^{i}\left(y_{(n+1) i} / \lambda\right)\right\}
\end{aligned}
$$

Observar que $f^{i}\left(y_{(n+1) i} / \lambda\right)$ é dado por,

$$
f^{\prime}\left(y_{(n+1) i} / \lambda\right)=\frac{\lambda^{1 / 2}}{\left(2 \pi y_{(n+1) i}^{3}\right)^{1 / 2}} \exp \left\{-\frac{\lambda}{2 y_{(n+1) i}}\left[\left(\alpha+\beta x_{i}\right) y_{(n+1) i}-1\right]^{2}\right\}
$$

onde $y_{(n+1) i}>0$ e $\lambda>0$.

Substituindo (5.8) em (5.7) temos, 
$r\left(y_{(n+1) i} /\right.$ dados $)=$

$=\int_{0}^{\infty} \frac{\lambda^{1 / 2}}{\left(2 \pi y_{(n+1) i}^{3}\right)^{1 / 2}} \exp \left\{-\frac{\lambda}{2 y_{(n+1) i}}\left\{\left(\alpha+\beta x_{i}\right) y_{(n+1) i}-1\right\}\right\} \frac{A(\alpha, \beta)^{n / 2}}{2^{n / 2} \Gamma\left(\frac{n}{2}\right)} \lambda^{n / 2-1} \exp \left\{-\frac{\lambda}{2} A(\alpha, \beta)\right\} d \lambda$

Definindo $B=\frac{1}{y_{(n+1) i}}\left[\left(\alpha+\beta x_{i}\right) y_{(n+1) i}-1\right]^{2}$, temos:

$$
f\left(y_{(n+1) i} / \text { dados }\right)=\frac{A(\alpha, \beta)^{n / 2}}{2^{n / 2} \Gamma\left(\frac{n}{2}\right) \sqrt{2 \pi} y_{(n+1) i}^{3 / 2}} \int_{0}^{\infty} \lambda^{(n-1) / 2} \exp \left\{-\frac{\lambda}{2}(A(\alpha, \beta)+B)\right\} d \lambda
$$

observar que a integral em (5.9) também pode ser resolvida com resultados conhecidos (ver Box e Tiao, 1973, pag.144).

Assim, a densidade preditiva para uma observação futura $Y_{(n+1) i}$ num nivel de estresse $i$, é dada por,

$$
\begin{aligned}
& f^{i}\left(y_{(n+1) i} / \text { dados }\right)=\frac{A(\alpha, \beta)^{n / 2}}{2^{n / 2} \Gamma\left(\frac{n}{2}\right) \sqrt{2 \pi} y_{(n+1) i}^{3 / 2}} \frac{\Gamma\left(\frac{n+1}{2}\right)}{\left(\frac{A(\alpha, \beta)+B}{2}\right)(n+1) / 2} \\
& =\frac{\Gamma\left(\frac{n+1}{2}\right) A(\alpha, \beta)^{n / 2}}{\sqrt{\pi} \Gamma\left(\frac{n}{2}\right) y_{(n+1) i}^{3 / 2}(A(\alpha, \beta)+B)^{(n+1) / 2}},
\end{aligned}
$$

isto $\dot{e}$,

$$
\begin{aligned}
& \text { isto é, } \\
& \dot{\mathrm{r}}\left(\mathrm{y}_{(n+1) i} / \text { dados }\right)=\frac{A(\alpha, \beta)^{n / 2} \Gamma\left(\frac{\mathrm{n}+1}{2}\right)}{\sqrt{\pi} \Gamma\left(\frac{n}{2}\right) \mathrm{y}_{(n+1) i}^{3 / 2}\left\{\mathrm{~A}(\alpha, \beta)-2\left(\alpha+\beta \mathrm{x}_{i}\right)+\left(\alpha+\beta \mathrm{x}_{i}\right)^{2} \mathrm{y}_{(n+1) i}+\mathrm{y}_{(n+1) i}^{-1}\right\}^{(n+1) / 2}}
\end{aligned}
$$

pois,

$$
\begin{aligned}
& \mathrm{A}(\alpha, \beta)+\mathrm{B}=\mathrm{A}(\alpha, \beta)+\frac{1}{y_{(n+1) i}}\left[\left(\alpha+\beta \mathrm{x}_{i}\right) \mathrm{y}_{(n+1) i}-1\right]^{2} \\
& =\mathrm{A}(\alpha, \beta)+\frac{1}{\mathrm{y}_{(n+1) i}}\left[\left(\alpha+\beta \mathrm{x}_{i}\right)^{2} \mathrm{y}_{(n+1) i}^{2}-2\left(\alpha+\beta \mathrm{x}_{i}\right) \mathrm{y}_{(n+1) i}+1\right] \\
& =\mathrm{A}(\alpha, \beta)+\left(\alpha+\beta \mathrm{x}_{i}\right)^{2} \mathrm{y}_{(n+1) i}-2\left(\alpha+\beta \mathrm{x}_{i}\right)+\frac{1}{\mathrm{y}_{(n+1) i}}
\end{aligned}
$$

onde $y_{(n+1) i}>0$.

Observamos que $\mathrm{n}$ é conhecido (dos dados usados no teste acelerado), $\mathrm{A}(\alpha, \beta)$ é conbecido, $\alpha$ e $\beta$ são conhecidos e $x_{i}$ é o nivel fixo da variável estresse. 


\subsection{USO DA DENSIDADE PREDITIVA EM CONTROLE DE QUALIDADE.}

Podemos usar a densidade preditiva fi $\left(y_{(n+1) i} /\right.$ dados) para formular procedimentos em controle de qualidade em testes de vida. Usualmente, engenheiros de qualidade selecionam amostras aleatórias de cada lote de componentes manufaturados para verificar se o processo em linha está sob controle. Para minimizar o custo e o tempo do teste, eles consideram unidades em testes de vida com altos niveis de estresse $X_{i}$ e um periodo fixo de tempo $L_{i}$.

Usando a densidade preditiva (5.10) com $\alpha$ e $\beta$ conhecidos, e considerando uma probabilidade fixa $1-\gamma$, podemos encontrar os valores requeridos de $X_{i}$ e $L_{i}$ para ter,

$$
P^{i}\left(Y_{(n+1) i}>L_{i} / \text { dados }\right)=1-\gamma
$$

De (5.10), temos,

$P^{i}\left(y_{(n+1) i}>L_{i} /\right.$ dados $)=\frac{\Gamma\left(\frac{n+1}{2}\right) A(\alpha, \beta)^{n / 2}}{\sqrt{\pi} \Gamma\left(\frac{n}{2}\right)} \int_{L_{i}}^{\infty} \frac{d y_{(n+1) i}}{y_{(n+1) i}^{3 / 2}\left\{c_{i}+d_{i} y_{(n+1) i}+y_{(n+1) i}^{-1}\right\}^{(n+1) / 2}}$

onde $c_{i}=A(\alpha, \beta)-2\left(\alpha+\beta x_{i}\right)$ ed $d_{i}=\left(\alpha+\beta x_{i}\right)^{2}$.

$\operatorname{De}(5.11)$ e (5.12) obtemos,

$$
\int_{L_{i}}^{\infty} \frac{d y_{(n+1) i}}{y_{(n+1) i}^{3 / 2}\left\{c_{i}+d_{i} y_{(n+1) i}+y_{(n+1) i}^{-1}\right\}^{(n+1) / 2}}=\frac{(1-\gamma) \sqrt{\pi} \Gamma\left(\frac{n}{2}\right)}{A(\alpha, \beta)^{n / 2} \Gamma\left(\frac{n+1}{2}\right)}
$$

De (5.13), podemos encontrar os valores requeridos de $X_{i}$ e $L_{i}$ para serem usados em testes de controle de qualidade, e consideramos o seguinte procedimento:

a) Colocamos $m$ unidades em teste com um nivel de estresse $X_{i}$ e durante um periodo de tempo $L_{i}$. Definimos $\mathrm{p}^{i}=\mathrm{P}^{i}\left(\mathrm{Y}_{(n+1) i} \leq \mathrm{L}_{i} /\right.$ dados) e consideramos a hipótese $\mathrm{H}_{0}: \mathrm{p}^{i} \leq \gamma$ (a linha de produção está sob contrôle) contra $\mathrm{H}_{1}: \mathrm{p}^{i}>\gamma(\mathrm{a}$ linha de produção está fora de contrôle).

b) Seja $\mathrm{X}$ o número de unidades que falham na amostra de tamanho $\mathrm{m}$, isto $\dot{e}$, as unidades tais que $Y \leq L_{i}$. Assumimos $X \sim b\left(m, p^{j}\right)$. Com $m$ grande (digamos $\left.m \geq 30\right)$, temos $X \stackrel{a}{\sim} N\left\{m p^{i}, m p^{i}\left(1-p^{i}\right)\right\}$. 
5.2 - uso da densidade preditiva em contróle de qualidade - pág. 61

c) Um criterio para controle de qualidade pode ser baseado num teste de hipóteses usual (com m grande): a produção em linha está fora de contrôle se $\mathrm{Z} \geq{ }_{2} \gamma$, onde $\gamma$ é o nivel de significâncie,

$$
\mathrm{Z}=\frac{(\mathrm{X}-\mathrm{m} \gamma)}{\sqrt{\mathrm{m \gamma}(1-\gamma)}} \stackrel{a}{\sim} \mathrm{N}\{0,1\}
$$

e $z_{\gamma}$ é tal que $P\left\{Z \geq z_{\gamma}\right\}=\gamma$.

Observar que para encontrarmos os valores de $X_{i}$ e $L_{i}$, devemos usar um método numérico (por exemplo, o método de Simpson, ver apêndice A2). 


\section{CAPÍTULO 6}

\section{PLANEJAMENTOS DE EXPERIMENTOS EM TESTES ACELERADOS COM O MODELO DE REGRESSÃO LINEAR INVERSO}

\subsection{UMA ANÁLISE CLÁSSICA.}

\subsubsection{FORMULAÇÃO DO MODELO.}

Considerar um teste de vida acelerado com dois fatores, o fator linha (A) com I niveis e o fator coluna (B) com $\mathrm{J}$ níveis. Em cada cela $(i, j), \mathbf{n}$ items são testados e seus tempos de falha $y_{i j k}$, $k=1,2, \ldots, \mathrm{n}$ são registrados. $\mathrm{O}$ experimento pode ser esquematizado na seguinte tabela:

\section{TABELA 6.1}

EXPERIMENTO FATORIAL COM DOIS FATORES

\begin{tabular}{|c|c|c|c|c|}
\hline $\begin{array}{r} \\
\quad \text { B } \\
\text { A }\end{array}$ & 1 & 2 & $\cdot \cdot \cdot \cdot$ & $\mathbf{J}$ \\
\hline 1 & $\underline{y}_{11}$ & $\underline{y}_{12}$ & $\underline{y}_{1 j}$ & $\underline{y}_{1 \mathrm{~J}}$ \\
\hline 2 & $\underline{y}_{21}$ & $\underline{y}_{22}$ & $\underline{y}_{2 j}$ & $\underline{y}_{2 \mathrm{~J}}$ \\
\hline $\begin{array}{l}\cdot \\
\cdot \\
.\end{array}$ & $\underline{y}_{i 1}$ & $\underline{y}_{i 2}$ & $\begin{array}{l}\mathbf{y}_{i j 1} \\
\mathbf{y}_{i j 2} \\
\vdots \\
y_{i j n}\end{array}$ & $\underline{y}_{i J}$ \\
\hline$I$ & $\mathbf{y}_{l 1}$ & $y_{I 2}$ & $\underline{y}_{I j}$ & $\underline{y}_{I J}$ \\
\hline
\end{tabular}


As observações são assumidas independentes com $y_{i j k}$ distribuidas como $\operatorname{IG}\left(\theta_{i j}, \sigma\right)$ com $\sigma=\lambda^{-1}$ com f.d.p. (2.1). Vamos considerar principalmente a modelo aditivo ou de não-interacão a qual assume que os tempos de primeira passagem do processo de Wiener correspondente para cada cela é a soma dos efeitos dos fatores. Como a média é inversamente proporcional para a passagem, a parametrização usual dos efeitos dos falores sugere o modelo,

$$
\theta_{i j}^{-1}=\mu+\alpha_{i}+\beta_{j}
$$

onde $\sum_{i=1}^{l} \alpha_{i}=\sum_{j=1}^{J} \beta_{j}=0$, e $\mu, \alpha_{i}$ e $\beta_{j}$ representam o efeito geral, o efeito linha e o efeito coluna respetivamente.

Para esta distribuição $\operatorname{IG}\left(\theta_{i j}, \sigma\right)$ temos $\theta_{i j}>0$ para todo $(i, j)$ e $\sigma>0$. Assim os paràmetros $\mu, \alpha^{\prime}=\left(\alpha_{1}, \alpha_{2}, \ldots, \alpha_{I}\right), \beta^{\prime}=\left(\beta_{1}, \beta_{2}, \ldots, \beta_{J}\right)$, e $\sigma$ estão no espaço paramétrico,

$$
\Omega=\left\{\left(\mu, \alpha^{\prime}, \beta^{\prime}, \sigma\right): \sum_{i=1}^{l} \alpha_{i}=\sum_{j=1}^{J} \beta_{j}=0, \mu+\alpha_{i}+\beta_{j}>0 \forall i, j ; \sigma>0\right\}
$$

Definimos as seguintes estatisticas básicas,

$$
\begin{aligned}
& y_{i j}=\sum_{k} y_{i j k}=n \bar{y}_{i j} \\
& y_{i .}=\sum_{j} y_{i j}=n J \bar{y}_{i .} \\
& y_{. j}=\sum_{i} y_{i j}=n I \bar{y}_{. j} \\
& y_{\cdot .}=\sum_{i} \sum_{j} y_{i j}=n I J \bar{y}_{. .} \\
& R^{*}=\sum_{i} \sum_{j} \sum_{k} y_{i j k}^{-1} \\
& D^{*}=\operatorname{diag}\left(\bar{y}_{11}, \bar{y}_{12}, \ldots, \bar{y}_{I J}\right) .
\end{aligned}
$$

Usamos também algumas propriedades amostrais da distribuição Gaussiana Inversa (ver Tweedie, 1957) dadas por: as estatísticas $\bar{y}_{i j}$ e $\sum_{k}\left(y_{i j k}^{-1}-\bar{y}_{i j}^{-1}\right)$ são independentes; a primeira é distribuida como $\operatorname{IG}\left(\theta_{i j}, \sigma / n\right)$ e a segunda dividida por $\sigma$ é distribuida como $\chi^{2}(\mathrm{n}-1)$. Além disso,

$$
\begin{aligned}
& \mathrm{E}\left(\overline{\mathrm{y}}_{i j}^{-1}\right)=\theta_{i j}^{-1}+\frac{\sigma}{\mathrm{n}} \\
& \mathrm{V}\left(\overline{\mathrm{y}}_{i j}^{-1}\right)=\theta_{i j}^{-1} \frac{\sigma}{\mathrm{n}}+2\left(\frac{\sigma}{\mathrm{n}}\right)^{2} .
\end{aligned}
$$




\subsubsection{ESTIMADORES DE MÁXIMA VEROSSIMILEANCA.}

De (2.1) e (6.1) a função de verossimilhança para $\theta$ e $\lambda$ é dada por,

$$
\begin{aligned}
& \mathrm{L}\left(\theta_{i j}, \sigma\right)=\prod_{i}^{I} \prod_{j}^{J} \prod_{k}^{n}\left(2 \pi \sigma \mathrm{y}^{3}\right)^{-1 / 2} \exp \left[-\frac{1}{2} \sigma^{-1} y_{i j k}^{-1}\left(y_{i j k} \theta_{i j}^{-1}-1\right)^{2}\right]= \\
& =(2 \pi)^{-I J n / 2} \sigma^{-I J n / 2} \prod_{i}^{I} \prod_{j}^{J} \prod_{k}^{n} y_{i j k}^{-3 / 2} \exp \left\{-\frac{1}{2} \sigma^{-1} \sum_{i}^{I} \sum_{j}^{J} \sum_{k}^{n} y_{i j k}^{-1}\left(y_{i j k} \theta_{i j}^{-1}-1\right)^{2}\right\} .
\end{aligned}
$$

O logaritmo da função de verossimilhança é dado por,

$$
\mathrm{l}\left(\theta_{i j}, \lambda\right) \propto-\frac{\mathrm{IJn}}{2} \log \sigma-\frac{1}{2} \sigma^{-1} \sum_{i}^{I} \sum_{j}^{J} \sum_{k}^{n} y_{i j k}^{-1}\left(y_{i j k} \theta_{i j}^{-1}-1\right)^{2}
$$

$\operatorname{com} \theta_{i j}^{-1}=\mu+\alpha_{i}+\beta_{j}$ em (6.1).

Assim,

$$
\mathrm{l}\left(\mu, \alpha_{i}, \beta_{j}, \sigma\right) \propto-\frac{\mathrm{IJn}}{2} \log \sigma-\frac{1}{2} \sigma^{-1} \sum_{i}^{1} \sum_{j}^{J} \sum_{k}^{n} \mathrm{y}_{i j k}^{-1}\left[\mathrm{y}_{i j k}\left(\mu+\alpha_{i}+\beta_{j}\right)-1\right]^{2}
$$

onde $\left[y_{i j k}\left(\mu+\alpha_{i}+\beta_{j}\right)-1\right]^{2}=y_{i j k}^{2}\left(\mu+\alpha_{i}+\beta_{j}\right)^{2}-2 y_{i j k}\left(\mu+\alpha_{i}+\beta_{j}\right)+1$.

Portanto,

$$
\begin{gathered}
\mathrm{l}\left(\mu, \alpha_{i}, \beta_{j}, \sigma\right) \propto-\frac{\mathrm{IJn}}{2} \log \sigma-\frac{1}{2} \sigma^{-1} \mu^{2} \sum_{i}^{I} \sum_{j}^{J} \sum_{k}^{n} y_{i j k}-\sigma^{-1} \mu \sum_{i}^{I} \sum_{j}^{J} \sum_{k}^{n} \alpha_{i} y_{i j k}-\sigma^{-1} \mu \sum_{i}^{I} \sum_{j}^{J} \sum_{k}^{n} \beta_{j} \mathrm{y}_{i j k}- \\
-\frac{1}{2} \sigma^{-1} \sum_{i}^{I} \sum_{j}^{J} \sum_{k}^{n} \alpha_{i}^{2} \mathrm{y}_{i j k}-\sigma^{-1} \sum_{i}^{I} \sum_{j}^{J} \sum_{k}^{n} \alpha_{i} \beta_{j} \mathrm{y}_{i j k}-\frac{1}{2} \sigma^{-1} \sum_{i}^{I} \sum_{j}^{J} \sum_{k}^{n} \beta_{j}^{2} \mathrm{y}_{i j k}+\sigma^{-1} \mu \mathrm{IJn}+ \\
+\sigma^{-1} \mathrm{Jn} \sum_{i}^{I} \alpha_{i}+\sigma^{-} \ln \sum_{j}^{J} \beta_{j}-\frac{1}{2} \sigma^{-1} \sum_{i}^{I} \sum_{j}^{J} \sum_{k}^{n} y_{i j k}^{-1}
\end{gathered}
$$

Observar que temos uma familia exponencial de distribuiçôes com as estatísticas suficientes $\left(D^{*}, R^{*}\right)$ de dimensão $(I J+1)$ onde o espaço paramétrico $\Omega$ é de dimensão $(I+J)$.

As primeiras derivadas de $\mathrm{l}\left(\mu, \alpha_{i}, \beta_{j}, \sigma\right)(6.6)$ em relação a $\mu, \alpha_{i}, \beta_{j}$ e $\sigma$ e igualadas a zero, são dadas por,

$$
\frac{\partial \mathrm{l}}{\partial \mu}=\mu \sum_{i}^{I} \sum_{j}^{J} \sum_{k}^{n} y_{i j k}+\sum_{i}^{I} \sum_{j}^{J} \sum_{k}^{n} \alpha_{i} \mathrm{y}_{i j k}+\sum_{i}^{I} \sum_{j}^{J} \sum_{k}^{n} \beta_{j} \mathrm{y}_{i j k}-\mathrm{IJn}=0
$$




$$
\begin{aligned}
& \frac{\partial 1}{\partial \alpha_{i}}=\mu \sum_{i}^{I} \sum_{j}^{J} \sum_{k}^{n} y_{i j k}+\sum_{i}^{I} \sum_{j}^{J} \sum_{k}^{n} \alpha_{i j} y_{i j k}+\sum_{i}^{J} \sum_{j}^{J} \sum_{k}^{n} \beta_{j} y_{i j k}-\mathrm{Jn}=0 \\
& \frac{\partial 1}{\partial \beta_{j}}=\mu \sum_{i}^{I} \sum_{j}^{J} \sum_{k}^{n} y_{i j k}+\sum_{i}^{I} \sum_{j}^{J} \sum_{k}^{n} \alpha_{i} y_{i j k}+\sum_{i}^{I} \sum_{j}^{J} \sum_{k}^{n} \beta_{j} y_{i j k}-\mathrm{In}=0
\end{aligned}
$$

e a primeira derivada com respeito a $\sigma$ de (6.5) é dada por,

isto $\dot{\mathbf{e}}$

$$
\frac{\partial \mathrm{l}}{\partial \sigma}=-\frac{\mathrm{IJn}}{2} \frac{1}{\sigma}+\frac{1}{2} \sigma^{-2} \sum_{i}^{I} \sum_{j}^{J} \sum_{k}^{n} y_{i j k}^{-1}\left[\mathrm{y}_{i j k}\left(\mu+\alpha_{i}+\beta_{j}\right)-1\right]^{2}=0
$$

$$
\dot{\sigma}=(\mathrm{IJn})^{-1} \sum_{i}^{I} \sum_{j}^{J} \sum_{k}^{n} y_{i j k}^{-1}\left[y_{i j k}\left(\mu+\alpha_{i}+\beta_{j}\right)-1\right]^{2}
$$

Portanto, as equações de máxima verossimilhança para $\mu, \alpha_{i}, \beta_{j}$ e $\sigma$ de (6.7), (6.8), (6.9) e (6.10) podem ser escritas a partir de (6.3) como,

$$
\begin{aligned}
& \hat{\mu} \mathrm{y}_{. .}+\sum_{i}^{l} \hat{\alpha}_{i} \mathrm{y}_{i .}+\sum_{j}^{J} \hat{\beta}_{j} \mathrm{y}_{. j}=\mathrm{IJn} \\
& \hat{\mu} \mathrm{y}_{i .}+\hat{\alpha}_{i} \mathrm{y}_{i .}+\sum_{j}^{J} \hat{\beta}_{j} \mathrm{y}_{i j}=\mathrm{Jn}, 1 \leq i \leq \mathrm{I} \\
& \hat{\mu} \mathrm{y}_{. j}+\sum_{i}^{l} \hat{\alpha}_{j} \mathrm{y}_{i j}+\hat{\beta}_{j} \mathrm{y}_{. j}=\mathrm{In}, 1 \leq j \leq \mathrm{J}
\end{aligned}
$$

e para $\sigma$ como,

$$
\hat{\sigma}=(\mathrm{IJn})^{-1} \sum_{i}^{l} \sum_{j}^{J} \sum_{k}^{n} \mathrm{y}_{i j k}^{-1}\left[\mathrm{y}_{i j k}\left(\hat{\mu}+\hat{\alpha}_{i}+\hat{\beta}_{j}\right)-1\right]^{2}
$$

onde as equações em (6.11a) são lineares nos parâmetros.

Para resolver (6.11) podemos obter uma representação alternativa dessas equações e usar esses resultados para mostrar que a raiz é única e que maximiza a função de verossimilhança. Para isso usamos as condições $\sum_{i}^{I} \alpha_{i}=\sum_{j}^{J} \beta_{j}=0$ para eliminar as últimas componentes de $\alpha$ e $\beta$, assim podemos definir os novos parâmetros como,

$$
\begin{aligned}
& \Phi=\left(\mu, \alpha_{1}, \alpha_{2}, \ldots, \alpha_{I-1}, \beta_{1}, \beta_{2}, \ldots, \beta_{J-1}\right)^{\prime}, \\
& \psi=\left(\Phi^{\prime}, \sigma\right)^{\prime}
\end{aligned}
$$

Para qualquer $(i, j)$, podemos identificar o $(I+J-1)$-vetor $x_{i j}$ consistindo de -1 's, $0^{\prime}$ 's e $1^{\prime}$ 's tais que $\mu+\alpha_{i}+\beta_{j}=\Phi^{\prime} x_{i j}, 1 \leq i \leq 1,1 \leq j \leq \mathrm{J} ;$ i.é.,

$$
\mu+\alpha_{i}+\beta_{j}=\left(\mu, \alpha_{1}, \alpha_{2}, \ldots, \alpha_{I-1}, \beta_{1}, \beta_{2}, \ldots, \beta_{J-1}\right)(1,0,0, \ldots, 1, \ldots, 0,0,0, \ldots, 1, \ldots, 0)^{\prime}
$$


Entāo,

$$
x_{i j}=(1,0,0, \ldots, 1, \ldots, 0,0,0, \ldots, 1, \ldots, 0)^{\prime}
$$

Portanto,

$$
\mu+\alpha_{i}+\beta_{j}=\Phi^{\prime} x_{i j}
$$

Observar que, $x_{I J}$ tem seu primeiro elemento igual a 1 e todos 08 outros iguais a -1 's, i.é.,

$$
\begin{gathered}
\mu+\alpha_{I}+\beta_{J}=\Phi^{\prime} \mathbf{x}_{I J}, \\
\operatorname{com} \alpha_{I}=-\alpha_{1}-\alpha_{2}-\ldots-\alpha_{I-1}, \text { e } \beta_{J}=-\beta_{1}-\beta_{2}-\ldots-\beta_{J-1} . \text { Então, } \\
\mathbf{x}_{I J}=(1,-1,-1, \ldots,-1,-1,-1, \ldots,-1)^{\prime} .
\end{gathered}
$$

Daí,

$$
\begin{aligned}
x_{11}= & (1,1,0, \ldots, 0,1,0, \ldots, 0)^{\prime} \\
x_{12}= & (1,0,1, \ldots, 0,0,1, \ldots, 0)^{\prime} \\
& \vdots \\
x_{i j}= & (1,0,0, \ldots, 1, \ldots, 0,0,0, \ldots, 1, \ldots, 0)^{\prime} \\
& \vdots \\
x_{I J}= & (1,-1,-1, \ldots,-1,-1,-1, \ldots,-1)^{\prime} .
\end{aligned}
$$

Assim, definimos as matrizes $\mathrm{X}$ e $\mathrm{M}$ de ordem $\mathrm{IJ} \times(I+\mathrm{J}-1)$ e $(I+\mathrm{J}-1) \times(I+J-1)$ respetivamente como,

$$
\begin{aligned}
& X^{\prime}=\left(x_{11}, x_{12}, \ldots, x_{I J}\right), \\
& M=X^{\prime} D^{*} X
\end{aligned}
$$

onde $D^{*}$ é dado em (6.3). Logo, temos que,

$$
\mathrm{X}^{\prime} 1=\mathrm{IJ}(1,0,0, \ldots, 0)^{\prime}=1 \mathrm{Je}=\delta
$$

onde $e^{\prime}=(1,0,0, \ldots, 0)^{\prime}$ é o vetor unitario. 
6.1.2 - Estimadores de máxima verossimilhanģa - pág. 67

De (6.6) ternos,

$$
\begin{aligned}
& \sum_{i}^{l} \sum_{j}^{J} \sum_{k}^{n} y_{i j k}\left(\mu+\alpha_{i}+\beta_{j}\right)^{2}=n \sum_{i}^{I} \sum_{j}^{J} \bar{y}_{i j}\left(\mu+\alpha_{i}+\beta_{j}\right)^{2}=\mathrm{n} \sum_{i}^{l} \sum_{j}^{J} \bar{y}_{i j}\left(\Phi^{\prime} x_{i j}\right)^{2}, \\
& \text { pois, } y_{i j}=\sum_{k}^{n} y_{i j k}=n \bar{y}_{i j} \text { e } \mu+\alpha_{i}+\beta_{j}=\Phi^{\prime} x_{i j} .
\end{aligned}
$$

Então,

$$
\sum_{i}^{I} \sum_{j}^{j} \sum_{k}^{n} y_{i j k}\left(\mu+\alpha_{i}+\beta_{j}\right)^{2}=n \Phi^{\prime} X^{\prime} D^{*} X \Phi=n \Phi^{\prime} M \Phi
$$

onde de (6.4) M é dado por,

$$
M=X^{\prime} D^{*} X=\left(x_{11}, x_{12}, \ldots, x_{I J}\right)\left[\begin{array}{cccc}
\bar{y}_{11} & & & 0 \\
& \bar{y}_{12} & & \\
& & \ddots & \\
0 & & & \bar{y}_{I J}
\end{array}\right]\left[\begin{array}{c}
x_{11}^{\prime} \\
x_{12}^{\prime} \\
\vdots \\
x_{I J}^{\prime}
\end{array}\right]
$$

assim,

$$
M=x_{11} x_{11}^{\prime} \bar{y}_{11}+x_{12} x_{12}^{\prime} \bar{y}_{12}+\ldots+x_{I J} x_{I J}^{\prime} \bar{y}_{I J}
$$

logo,

$$
M=\sum_{i}^{I} \sum_{j}^{J} x_{i j} x_{i j}^{\prime} \bar{y}_{i j}=X^{\prime} D^{*} X
$$

Também,

$$
\begin{aligned}
& \Phi^{\prime} \mathbf{M} \Phi=\Phi^{\prime}\left[\sum_{i}^{I} \sum_{j}^{J}\left(x_{i j} x_{i j}^{\prime}\right) \bar{y}_{i j}\right] \Phi \\
& =\sum_{i}^{I} \sum_{j}^{J}\left(\Phi^{\prime} x_{i j} \mathbf{x}_{i j}^{\prime} \Phi\right) \bar{y}_{i j} \\
& =\sum_{i}^{I} \sum_{j}^{J}\left(\Phi^{\prime} x_{i j}\right)^{2} \bar{y}_{i j}
\end{aligned}
$$

Portanto,

$$
\sum_{i}^{I} \sum_{j}^{J} \sum_{k}^{n} y_{i j k}\left(\mu+\alpha_{i}+\beta_{j}\right)^{2}=n \Phi^{\prime} M \Phi .
$$

Agora,

$$
\begin{aligned}
& -2 \sum_{i}^{I} \sum_{j}^{J} \sum_{k}^{n}\left(\mu+\alpha_{i}+\beta_{j}\right)=-2 \mathrm{n} \sum_{i}^{I} \sum_{j}^{J}\left(\mu+\alpha_{i}+\beta_{j}\right)=-2 \mathrm{n}\left\{\mathrm{IJ} \mu+\sum_{j}^{J}\left(\sum_{i}^{I} \alpha_{i}\right)+\sum_{i}^{I}\left(\sum_{j}^{J} \beta_{j}\right)\right\} \\
& \operatorname{com} \sum_{i}^{I} \alpha_{i}=\sum_{j}^{J} \beta_{j}=0 . \text { Então, }
\end{aligned}
$$




$$
-2 \mathrm{n} \sum_{i}^{I} \sum_{j}^{J}\left(\mu+\alpha_{i}+\beta_{j}\right)=-2 \mathrm{nIJ} \mu
$$

isto é,

$$
-2 \mathrm{nIJ} \mu=-2 \mathrm{n} \Phi^{\prime} \delta
$$

$\operatorname{com} \Phi=\left(\mu, \alpha_{1}, \alpha_{2}, \ldots, \alpha_{I-1}, \beta_{1}, \beta_{2}, \ldots, \beta_{J-1}\right)^{\prime} \in \delta=(\mathrm{IJ}, 0, \ldots, 0)^{\prime}$

Portanto,

$$
-2 \sum_{i}^{I} \sum_{j}^{J} \sum_{k}^{n}\left(\mu+\alpha_{i}+\beta_{j}\right)=-2 n \Phi^{\prime} \delta
$$

Assim (6.6) pode ser escrita por,

$$
\mathrm{I}(\Phi, \sigma)=-\frac{1}{2} \mathrm{IJn} \log \sigma-\frac{1}{2} \sigma^{-1}\left\{\mathrm{R}^{*}-2 \mathrm{n} \Phi^{\prime} \delta+\mathrm{n} \Phi^{\prime} M \Phi\right\}
$$

De (6.15) podemos obter os EMV que são dados por (os resultados da diferenciação de matrizes podem ser encontrados em Morrison (1967), pág. 68),

$$
\begin{aligned}
& \frac{\partial 1}{\partial \Phi}=\frac{\mathrm{n}}{\sigma}[\delta-\mathbf{M} \Phi] \\
& \frac{\partial 1}{\partial \sigma}=-\frac{\mathrm{IJn}}{2 \sigma}+\frac{1}{2 \sigma^{2}}\left\{R^{*}-2 \mathrm{n} \Phi^{\prime} \delta+n \Phi^{\prime} M \Phi\right\}
\end{aligned}
$$

onde $M$ é definida positiva pois a matriz diagonal $D^{*}$ é positiva definida, logo os $y_{i j}^{\prime}$ são positivos. Assim os resultados padrões para $o$ experimento com dois fatores exigem que o $\operatorname{posto}\left(\mathbf{X}^{\prime}\right)=\operatorname{posto}\left(\mathbf{X}^{\prime} \mathbf{X}\right)=\mathrm{I}+\mathrm{J}-1$. Deste modo, somente o vetor $\boldsymbol{\omega}$ satisfazendo $\mathbf{X} \boldsymbol{\omega}=\mathbf{0}$ é o vetor nulo. Consequentemente, para um $\omega \neq 0$ arbitrario, temos $\omega^{\prime} \mathbf{M} \omega=(X \omega)^{\prime} D^{*}(X \omega)>0$, isto é, $M$ é positiva definida.

As soluçōes correspondentes das equações de verossimilhança de (6.16) e (6.17) são,

$$
\begin{aligned}
& \hat{\Phi}=M^{-1} \delta \\
& \hat{\sigma}=(I J n)^{-1}\left[R^{*}-n \delta^{\prime} M^{-1} \delta\right] .
\end{aligned}
$$

A matriz de informação observada $I_{0}$ é dada por,

$$
\mathfrak{I}_{0}=-\left.\frac{\left.\partial^{2}\right\rfloor}{\partial \psi \partial \boldsymbol{\psi}^{\prime}}\right|_{\psi}=\dot{\psi}
$$


Assim as segundas derivadas parciais com sinal negativo da função log-verossimilhança (6.16) são dadas por,

$$
\begin{aligned}
& -\frac{\partial^{2} l}{\partial \Phi^{2}}=n M \sigma^{-1} \\
& -\frac{\partial^{2} l}{\partial \sigma^{2}}=\frac{1}{\sigma^{3}}\left[R^{\prime \prime}-2 n \Phi^{\prime}+n \Phi^{\prime} M \Phi\right]-\frac{1 \mathrm{Jn}}{2 \sigma^{2}} \\
& \left.-\frac{\left.\partial^{2}\right]}{\partial \Phi \partial \sigma}=\frac{n}{\sigma^{2}} \delta-M \Phi\right]=-\frac{\partial^{2} l}{\partial \sigma \partial \Phi}
\end{aligned}
$$

Calculando (6.20), (6.21) e (6.22) em $\dot{\Phi}$ e $\hat{\sigma}$ temos,

$$
\begin{aligned}
& -\left.\frac{\partial^{2} l}{\partial \Phi^{2}}\right|_{\psi=\dot{\psi}}=\mathrm{nM} \hat{\sigma}^{-1} \\
& -\left.\frac{\partial^{2} l}{\partial \sigma^{2}}\right|_{\psi=\dot{\psi}}=\frac{\mathrm{IJn}}{2 \dot{\sigma}^{2}} \\
& -\left.\frac{\partial^{2} l}{\partial \Phi \partial \sigma}\right|_{\psi=\dot{\psi}}=0=-\left.\frac{\partial^{2} \mathrm{l}}{\partial \sigma \partial \Phi}\right|_{\psi=\dot{\psi}}
\end{aligned}
$$

Assim, substituindo (6.23), (6.24) e (6.25) em (6.19) temos que a matriz de informação observada $\mathrm{I}_{0} \dot{e}$ dada como,

$$
\mathrm{I}_{0}=\mathrm{n}\left[\begin{array}{cc}
\mathbf{M} \hat{\sigma}^{-1} & 0 \\
0 & \frac{1}{2} \mathrm{JJ} \hat{\sigma}^{-2}
\end{array}\right]
$$

Esta matriz é positiva definida se $M$ for positiva definida e $\hat{\sigma}>0$, como pode ser vista na expressão (6.13). Portanto, $\hat{\boldsymbol{\phi}}$ maximiza a função de verossimilhança.

Para exigir que $\dot{\psi}$ seja o estimador de máxima verossimilhança precisamos mostrar que as raizes se encontram dentro do espaço paramétrico $\Omega$, isto é $\theta_{i j}^{-1}=\dot{\phi}^{\prime} \mathbf{x}_{i j}>0$ para todo $i, j$. Estes resultados são válidos para o caso especial $I=J=2$ (Fries, 1982), mas não é possível provar a generalização. No entanto, $\dot{\psi}$ fornece um estimador eficiente de máxima verossimilhança (Lehmann, 1980). Além disso, $\bar{\psi}$ é único e podemos dizer que quando $n$ é grande, o EMV $\hat{\psi}$ converge para $\psi$ com probabilidade 1.

As propriedades assintóticas $(\mathrm{n} \rightarrow \infty$ com I e J fixos) de $\hat{\psi}$ são dadas no teorema 1 . 
TEOREMA 1. Os estimadores $\dot{\phi}$ e $\hat{\sigma}$ são fortemente consistentes, e as distribuiçōes limites de $n^{1 / 2}(\dot{\Phi}-\Phi)$ e $n^{1 / 2}(\hat{\sigma}-\sigma)$ são $N_{l+J-1}\left(0, \sigma \Gamma^{-1}\right)$ e $N_{1}\left(0,2 \sigma^{2}(I J)^{-1}\right)$, respectivamente, onde

$$
\Gamma=X^{\prime} \theta X, \quad \theta=\operatorname{diag}\left(\theta_{11}, \theta_{12}, \ldots, \theta_{l J}\right)
$$

Além disso, $\bar{\Phi}$ e $\dot{\sigma}$ são assintoticamente independentes (Fries e Bhattacharyya, 1983).

\subsubsection{ESTIMADORES NÃO-VICIADOS VIA MÍNIMOS QUADRADOS.}

Ainda que na seção 6.2 encontramos expressōes fechadas para os estimadores de máxima verossimilhança, uma análise de seus vicios e variâncias exatas são difíceis porque esses estimadores envolvem a inversa de uma matriz aleatória. Nesta seção apresentamos uma aproximação via mínimos quadrados baseados sobre uma redução inicial dos dados por suficiência, fornecendo assim estimadores não-viciados como também expressōes exatas para suas variâncias e covariâncias ao contrário dos resultados assintóticos obtidos para $\hat{\boldsymbol{\phi}} \mathbf{e} \hat{\boldsymbol{\sigma}}$.

Definimos $\mathbf{r}_{i j}=\bar{y}_{i j}^{-1}$ como o inverso da média amostral em cada cela, os momentos resultantes são dados a partir de (6.4) i. é.,

$$
\begin{aligned}
& \mathrm{E}\left(\mathrm{r}_{i j}\right)=\mu+\alpha_{i}+\beta_{j}+\frac{\sigma}{\mathrm{n}} \\
& \mathrm{V}\left(\mathrm{r}_{i j}\right)=\left(\mu+\alpha_{i}+\beta_{j}\right) \frac{\sigma}{\mathrm{n}}+2\left(\frac{\sigma}{\mathrm{n}}\right)^{2} .
\end{aligned}
$$

Escrevendo,

$$
\begin{aligned}
& \mu^{*}=\mu+\frac{\sigma}{\mathrm{n}}, \\
& \psi^{*}=\left(\mu^{*}, \alpha^{\prime}, \beta^{\prime}\right)^{\prime},
\end{aligned}
$$

$\operatorname{com} \alpha^{\prime}=\left(\alpha_{1}, \alpha_{2}, \ldots, \alpha_{I}\right), \beta^{\prime}=\left(\beta_{1}, \beta_{2}, \ldots, \beta_{J}\right)$.

Temos o modelo linear dado por,

$$
\mathbf{r}=\mathbf{X} \psi^{*}+\epsilon, \operatorname{com} \mathrm{E}(\boldsymbol{\epsilon})=0,
$$

onde $r=\left(r_{11}, r_{12}, \ldots, r_{1 J}\right)^{\prime}$. 
Isto é similar a modelo linear usual para um experimento com dois fatores exceto que os inversos da media amostral em cada cela são utilizadas, e as variâncias dos $\mathbf{r}_{i j}$ ¿ são funçōes do vetor de parâmetros $\psi^{*}$. Também, os parâmetros originais $\mu$ e $\sigma$ são confundidos através de $\mu^{*}$.

Assim temos a seguinte reparametrização,

$$
\begin{aligned}
& \mathbf{r}_{i j}=\mu+\alpha_{i}+\beta_{j}+\frac{\sigma}{n}+\epsilon_{i j} \\
& =\mu^{*}+\alpha_{i}+\beta_{j}+\epsilon_{i j},
\end{aligned}
$$

com $i=1,2, \ldots, \mathrm{I}, j=1,2, \ldots, \mathrm{J}$ e $\alpha_{I}=-\alpha_{1}-\alpha_{2}-\ldots-\alpha_{I-1}, \beta_{J}=-\beta_{1}-\beta_{2}-\ldots-\beta_{J-1}$.

Isto é,

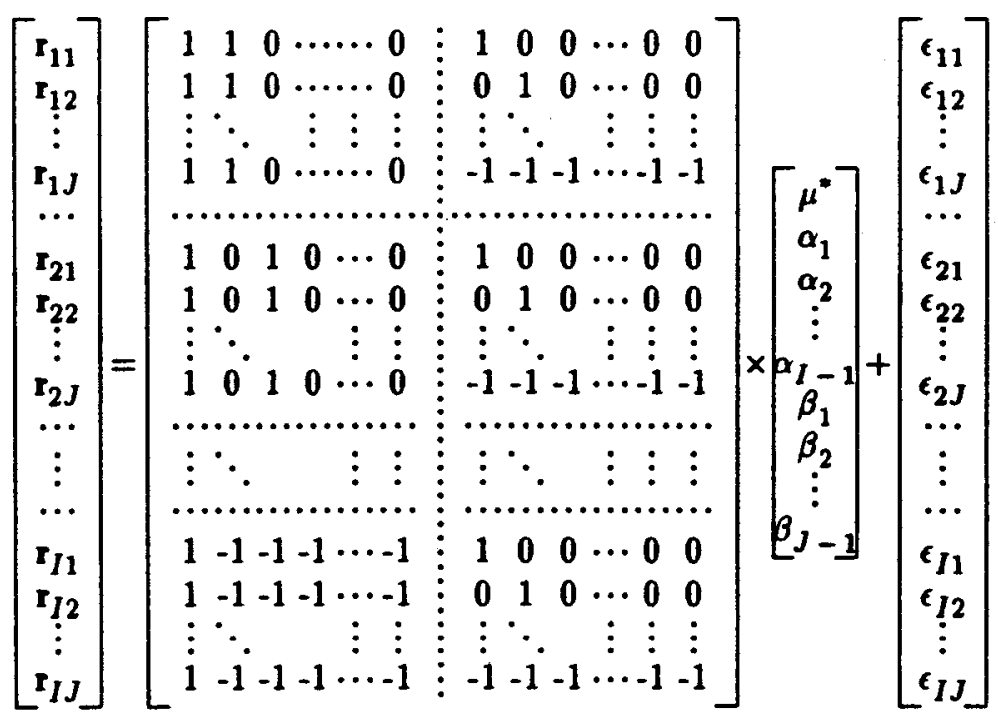

Portanto,

$$
\mathbf{r}=\mathbf{X} \boldsymbol{\psi}^{*}+\boldsymbol{\epsilon}
$$

Os resultados padrões para o experimento de dois fatores fornecem estimadores não viciados lineares numa forma simples dados por,

$$
\tilde{\mu}^{*}=\overline{\mathbf{r}}_{. .}, \tilde{\alpha}_{i}=\overline{\mathbf{r}}_{i .}-\overline{\mathbf{r}}_{. .}, \tilde{\beta}_{j}=\overline{\mathbf{r}}_{. j}
$$

com

$$
\overline{\mathbf{r}}_{. .}=\frac{\sum_{i}^{I} \sum_{j}^{J} \mathbf{r}_{i j}}{\mathrm{nIJ}}, \overline{\mathbf{r}}_{i .}=\frac{\sum_{j}^{J} \mathbf{r}_{i j}}{\mathrm{~nJ}}, \overline{\mathbf{r}}_{. j}=\frac{\sum_{i}^{I} \mathbf{r}_{i j}}{\mathrm{nI}} .
$$

Além disso, os parâmetros $\sigma$ e $\mu$ são estimados pelos estimadores não viciados de $\tilde{\sigma}$ e $\tilde{\mu}$ respetivamente, 
dados por,

$$
\tilde{\sigma}=M R_{E}, \tilde{\mu}=\overline{\mathbf{r}}_{. .}-\left(\frac{\tilde{\sigma}}{\bar{n}}\right)
$$

O estimador de $\dot{\psi}^{*}$ é dada por, $\tilde{\psi}^{*}=\left(X^{\prime} X\right)^{-1} X^{\prime} r$, desde que $\left(X^{\prime} X\right)^{\prime}$ existe.

Sua variância é dada por,

$$
\mathbf{V}\left(\tilde{\boldsymbol{\phi}}^{*}\right)=\left(\mathbf{X}^{\prime} \mathbf{X}\right)^{-1} \tilde{\boldsymbol{\sigma}}^{2}
$$

onde $\sigma^{2}$ é estimado por $\tilde{\sigma}^{2}=\frac{\mathbf{r}^{\prime} \mathbf{r}-\bar{\psi}^{*} X^{\prime} \mathbf{r}}{\mathrm{n}-\mathbf{p}}$.

Este método de estimação não fornece necessariamente estimadores positivos para as médias em cada cela $\theta_{i j}$ como é requerido pela definição do espaço paramétrico da distribuiçâo IG. No entanto, esses estimadores são altamente consistentes e assintoticamente normais. As distribuições limites são dadas no teorema 2.

TEOREMA 2. As distribuições limites de $n^{1 / 2}(\bar{\Phi}-\Phi)$ e $n^{1 / 2}(\hat{\sigma}-\sigma)$ são $N_{I+J-1}\left(0, \sigma \Gamma_{1}\right)$ e $N_{1}(0$, $\left.2 \sigma^{2}(\mathrm{IJ})^{-1}\right)$, respectivamente, onde

$$
\Gamma_{1}=V^{\prime} \theta^{-1} V, \quad V^{\prime}=\left(X^{\prime} X\right)^{\prime} X
$$

onde $\theta=\operatorname{diag}\left(\theta_{11}, \theta_{12}, \ldots, \theta_{I J}\right)$

Além disso, $\hat{\Phi}$ e $\dot{\sigma}$ são assintoticamente independentes (Fries e Bhattacharyya, 1983). 
6.2 UMA ANÁLISE BAYESIANA.

\subsubsection{DENSIDADE A PRIORI NÃO-INFORMATTVA PARA $\Phi$ E $\sigma$.}

Assumindo independência entre $\Phi$ e $\sigma$, consideremos uma densidade a priori não informativa conjunta (Box e Tiao, 1973) dada por,

$$
\pi(\Phi, \sigma) \propto \sigma^{-1}
$$

$\operatorname{com} \Phi=\left(\mu, \alpha_{1}, \alpha_{2}, \ldots, \alpha_{I-1}, \beta_{1}, \beta_{2}, \ldots, \beta_{J-1}\right)^{\prime}$, onde $-\infty<\mu<\infty,-\infty<\alpha_{i}<\infty,-\infty<\beta_{j}<\infty$ e $\sigma>0$.

\subsubsection{DENSIDADE A POSTERIORI CONJUNTA PARA $\Phi$ E $\sigma$.}

A função de verossimilhança para $\Phi$ e $\sigma$ é dada por,

$$
\mathrm{L}(\Phi, \sigma) \propto \sigma^{-\frac{1}{2} I J n} \exp \left\{-\frac{1}{2} \sigma^{-1}\left[\mathrm{R}^{*}-2 \mathrm{n} \Phi^{\prime} \delta+\mathrm{n} \Phi^{\prime} \mathrm{M} \Phi\right]\right\}
$$

Considerando (6.33) e (6.34), a densidade a posteriori conjunta para $\Phi$ e $\sigma$ é dada por,

$$
\pi(\Phi, \sigma) \propto \sigma^{-\left(\frac{I J n}{2}+1\right)} \exp \left\{-\frac{1}{2} \sigma^{-1}\left[R^{*}-2 n \Phi^{\prime} \delta+n \Phi^{\prime} M \Phi\right]\right\}
$$

onde $\Phi=\left(\mu, \alpha_{1}, \alpha_{2}, \ldots, \alpha_{I-1}, \beta_{1}, \beta_{2}, \ldots, \beta_{J-1}\right)^{\prime}$, com $-\infty<\mu<\infty,-\infty<\alpha_{i}<\infty$, $-\infty<\beta_{j}<\infty$ e $\sigma>0$.

\subsubsection{DENSIDADE A POSTERIORI MARGINAL PARA $\Phi$.}

A densidade a posteriori marginal conjunta para $\Phi$ de (6.36) é dada por,

$$
\pi(\Phi / \text { dados }) \propto \int_{0}^{\infty} \sigma^{-\left(\frac{I J n}{2}+1\right)} \exp \left\{-\frac{1}{2} \sigma^{-1}\left[R^{*}-2 n \Phi^{\prime} \delta+n \Phi^{\prime} \delta\right]\right\} d \sigma
$$

Para resolver esta integral, usamos resultados conhecidos (ver, Box e Tiao, 1973, pág. 144). Portanto, a densidade a posteriori marginal para $\Phi$ é dada por, 
6.2.4 - Densidade a posteriori marginal para $\sigma$ - pág. 74

$$
\pi(\Phi / \text { dados }) \propto\left[R^{*}-2 n \Phi^{\prime} \delta+n \Phi^{\prime} M \Phi\right]^{-\frac{l J n}{2}}
$$

onde $\Phi=\left(\begin{array}{llllllll}\mu & \alpha_{1}, & \alpha_{2}, \ldots, & \alpha_{I-1}, & \beta_{1}, & \beta_{2}, \ldots, & \beta_{J-1}\end{array}\right)^{\prime}, \operatorname{com}-\infty<\mu<\infty,-\infty<\alpha_{i}<\infty$, $-\infty<\beta_{j}<\infty$.

\subsubsection{DENSIDADE A POSTERIORI MARGINAL PARA $\sigma$.}

A densidade a posteriori marginal para $\sigma$ de (6.35) é dada por,

$$
\pi(\sigma / \text { dados }) \propto \int_{0}^{\infty} \sigma^{-\left(\frac{1 J n}{2}+1\right)} \exp \left\{-\frac{1}{2} \sigma^{-1}\left[R^{*}-2 n \Phi^{\prime} \delta+n \Phi^{\prime} \delta\right]\right\} d \Phi
$$

Para resolver esta integral, usamos o método de Laplace ( caso multiparamétrico, Apêndice A1), com,

$$
-n h(\Phi)=-\frac{1}{2} \sigma^{-1}\left[R^{*}-2 n \Phi^{\prime} \delta+n \Phi^{\prime} M \Phi\right], \text { e } f(\Phi)=1 \text { (ver A1.1 no apèndice A1). }
$$

Portanto,

$$
\pi(\sigma / \text { dados }) \propto \sigma^{-\left(\frac{I J n+1}{2}\right)} \exp \left\{-\frac{1}{2} \sigma^{-1}\left[\mathrm{R}^{*}-\mathrm{n} \delta^{\prime} \mathbf{M}^{-1} \delta\right]\right\}
$$

onde $\sigma>0$.

\subsubsection{INTERVALOS DE CREDIBILIDADE PARA OS PARÂMETROS.}

Para a construção de ICR para os parâmetros $\mu, \alpha_{i}, \beta_{j}$ e $\sigma$ fazemos uso das distribuições assintóticas normais para os estimadores obtidos. Assim para os EMV $\hat{\mu}, \hat{\alpha}_{i}, \hat{\beta}_{j}$ e $\hat{\sigma}$ usamos a aproximação normal dos EMV com matriz de variância-covariância assintótica dada pela inversa da matriz de informação observada (6.26). Da mesma forma, consideramos aproximaçōes normais para as densidades a posteriori (ver A3.2, Apêndice A3) de interêsse. 


\title{
CAPÍTULO 7
}

\author{
APLICAÇÕES NUMÉRICAS
}

\subsection{ANÁLISE DO MODELO GAUSSIANO INVERSO SEM VARIÁVEL ESTRESSE}

\subsubsection{PRIMEIRO EXEMPLO.}

$\mathrm{Na}$ tabela 7.1, apresentamos um conjunto de dados introduzido por Achcar, Bolfarine e Rodrigues (1987) os quais foram simulados a partir de uma distribuição Gaussiana inversa com parâmetros $\theta=1$ e $\lambda=5$.

TABELA 7.1

DADOS GERADOS DE UMA IG $(\theta, \lambda) \operatorname{COM} \theta=1$ e $\lambda=5(n=30)$

\begin{tabular}{|llllll|}
\hline 0.26 & 0.41 & 0.22 & 0.69 & 0.53 & 0.66 \\
0.56 & 0.52 & 0.80 & 1.00 & 1.03 & 0.81 \\
0.79 & 0.87 & 0.95 & 0.97 & 0.79 & 0.83 \\
0.83 & 1.29 & 1.11 & 1.43 & 1.23 & 1.81 \\
1.73 & 1.67 & 1.63 & 1.23 & 2.36 & 2.10 \\
\hline
\end{tabular}

\subsubsection{SEGUNDO EXEMPLO.}

A tabela 7.2 se refere a um conjunto de dados apresentados por Lieblin e Zelen (1956), que representa a resistência de encaixe de rolamentos dados em milhões de rotaçóes até falhar para cada um dos 23 rolamentos usados no teste de vida. 
7.1 - análise do modelo Gaussiano Inverso sem variável estresse - pág. 76

TABELA 7.2

RESISTÊNCIA DE ENCAIXE DE ROLAMENTOS (milhöes de rotaçōes)

\begin{tabular}{|llllll|}
\hline 17.88 & 28.92 & 33.00 & 41.52 & 42.12 & 45.60 \\
48.48 & 51.84 & 51.96 & 54.12 & 55.56 & 67.80 \\
68.64 & 68.64 & 68.88 & 84.12 & 93.12 & 98.64 \\
105.12 & 105.84 & 127.92 & 128.04 & 173.40 & \\
\hline
\end{tabular}

Estes dados foram analisados previamente assumindo uma distribuição Weibull ou Lognormal.

ANÁLISE CLÁSSICA - PARAMETRIZAÇÃO $(\theta, \lambda)$.

Para os conjuntos de dados dos exemplos 1 e 2, temos os seguintes resultados:

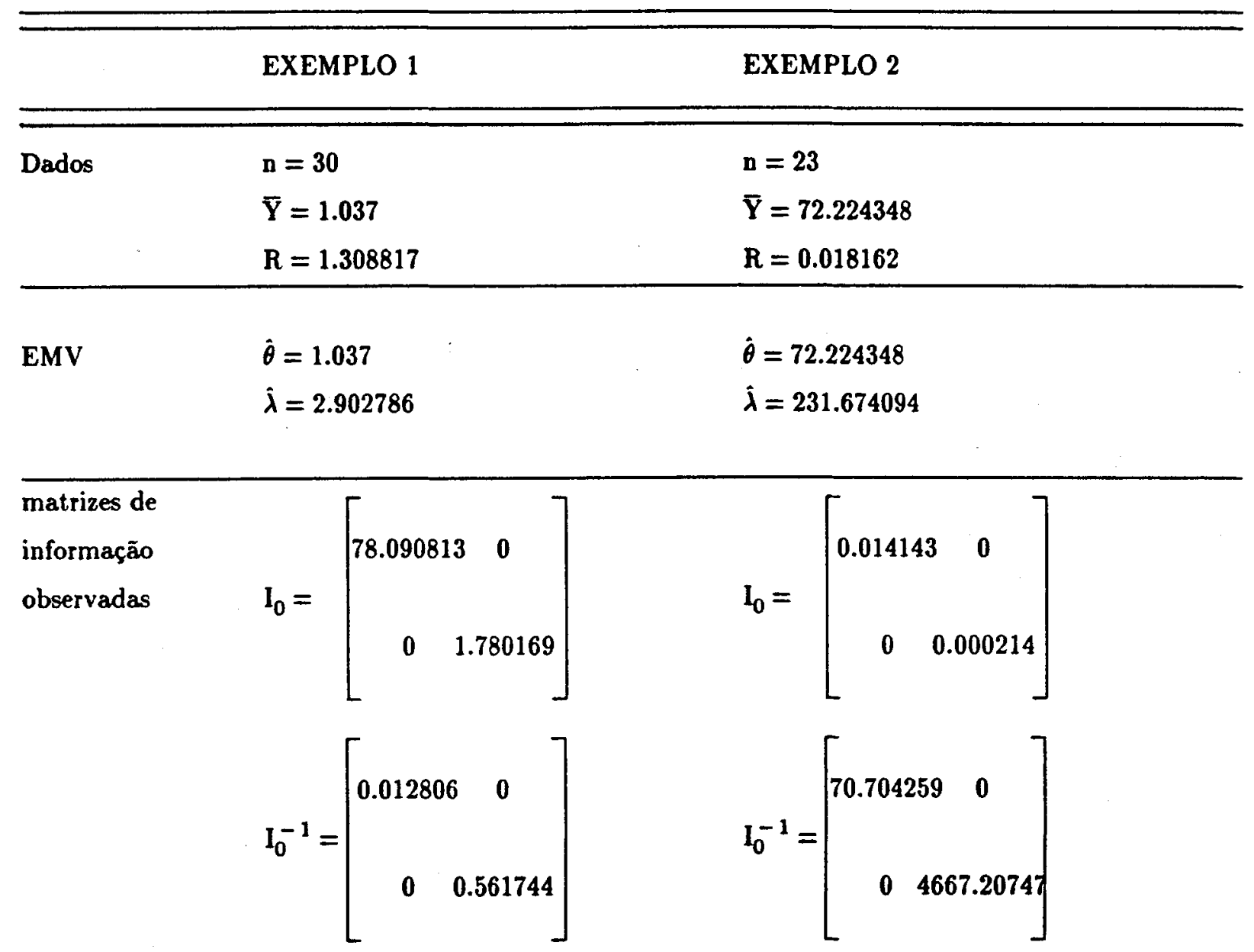


7.1 - análise do modelo Gaussiano Inverso sem variável estresse - pág. 77
variäncias
$\hat{\mathrm{V}}(\hat{\theta})=0.012806$
$\hat{\mathrm{V}}(\hat{\theta})=70.704259$
estimadas
$\dot{\mathrm{V}}(\bar{\lambda})=0.561744$
$\dot{V}(\dot{\lambda})=\mathbf{4 6 6 7 . 2 0 7 4 7}$

resultados análogos foram encontrados usando a matriz de informação de Fisher

Um teste do ajuste do modelo foi feito separadamente para cada um dos exemplos, utilizando a estatística de Kolmogorov-Smirnov (seção 2.1.1). Os resultados encontrados foram,

\section{EXEMPLO1.}

TABELA 7.3

DISTRIBUIÇÕES DE FREQUÊNCIA EMPÍRICA E OBSERVADA

\begin{tabular}{lllll}
\hline $\mathrm{i}$ & $\mathrm{F}(\mathrm{x})$ & $\mathrm{S}(\mathrm{x})$ & $\mathrm{i} / \mathrm{n}$ & $(\mathrm{i}-1) / \mathrm{n}$ \\
\hline 1 & & & & \\
2 & 0.003547 & 0.016667 & 0.033333 & 0.000000 \\
3 & 0.010098 & 0.050000 & 0.066667 & 0.033333 \\
4 & 0.081497 & 0.083333 & 0.100000 & 0.066667 \\
5 & 0.171926 & 0.116667 & 0.133333 & 0.100000 \\
6 & 0.181033 & 0.150000 & 0.166667 & 0.133333 \\
7 & 0.208849 & 0.183333 & 0.200000 & 0.166667 \\
8 & 0.303808 & 0.216667 & 0.233333 & 0.200000 \\
9 & 0.332095 & 0.250000 & 0.266667 & 0.233333 \\
10 & 0.422854 & 0.283333 & 0.300000 & 0.266667 \\
11 & 0.422854 & 0.316667 & 0.333333 & 0.300000 \\
12 & 0.431536 & 0.350000 & 0.366667 & 0.333333 \\
13 & 0.440134 & 0.383333 & 0.400000 & 0.366667 \\
14 & 0.457076 & 0.416667 & 0.433333 & 0.400000 \\
15 & 0.457076 & 0.450000 & 0.466667 & 0.433333 \\
16 & 0.489892 & 0.483333 & 0.500000 & 0.466667 \\
17 & 0.551043 & 0.516667 & 0.533333 & 0.500000 \\
18 & 0.565375 & 0.550000 & 0.566667 & 0.533333 \\
19 & 0.586157 & 0.583333 & 0.600000 & 0.566667 \\
& 0.606086 & 0.616667 & 0.633333 & 0.600000
\end{tabular}


7.1 - análise do modelo Gaussiano Inverso sem variável estresse - pág. 78

\begin{tabular}{lllll}
20 & 0.655162 & 0.650000 & 0.666667 & 0.633333 \\
21 & 0.718376 & 0.683333 & 0.700000 & 0.666667 \\
22 & 0.718376 & 0.716667 & 0.733333 & 0.700000 \\
23 & 0.745723 & 0.750000 & 0.766667 & 0.733333 \\
24 & 0.799928 & 0.783333 & 0.800000 & 0.766667 \\
25 & 0.858163 & 0.816667 & 0.833333 & 0.800000 \\
26 & 0.867598 & 0.850000 & 0.866667 & 0.833333 \\
27 & 0.880583 & 0.883333 & 0.900000 & 0.866667 \\
28 & 0.895924 & 0.916667 & 0.933333 & 0.900000 \\
29 & 0.936654 & 0.950000 & 0.966667 & 0.933333 \\
30 & 0.959271 & 0.983333 & 1.000000 & 0.966667 \\
\hline
\end{tabular}

As estatísticas de K-S obtidas (ver seção 2.1.1) foram,

$$
\begin{aligned}
& \mathrm{D}_{o b s}^{K S+}=0.0566 \text { e } \mathrm{D}_{o b s}^{K S-}=0.1562, \text { portanto } \\
& \mathrm{D}_{o b s}^{K S}=0.1562 \text { e } \phi=2.7992 .
\end{aligned}
$$

Assim, fixando $\gamma=0.05$ temos, $\mathrm{D}_{c r i}^{K S}=0.1660$, portanto $\mathrm{D}_{o b s}^{K S}<\mathrm{D}_{c r i}^{K S}$, indicando um bom ajuste do modelo Gaussiano inverso (ver gráfico 7.1).

\section{GRÁFICO 7.1}

\section{GRAFICO PROBABILISTICO - EMPIRICO US. OBSERUADO}

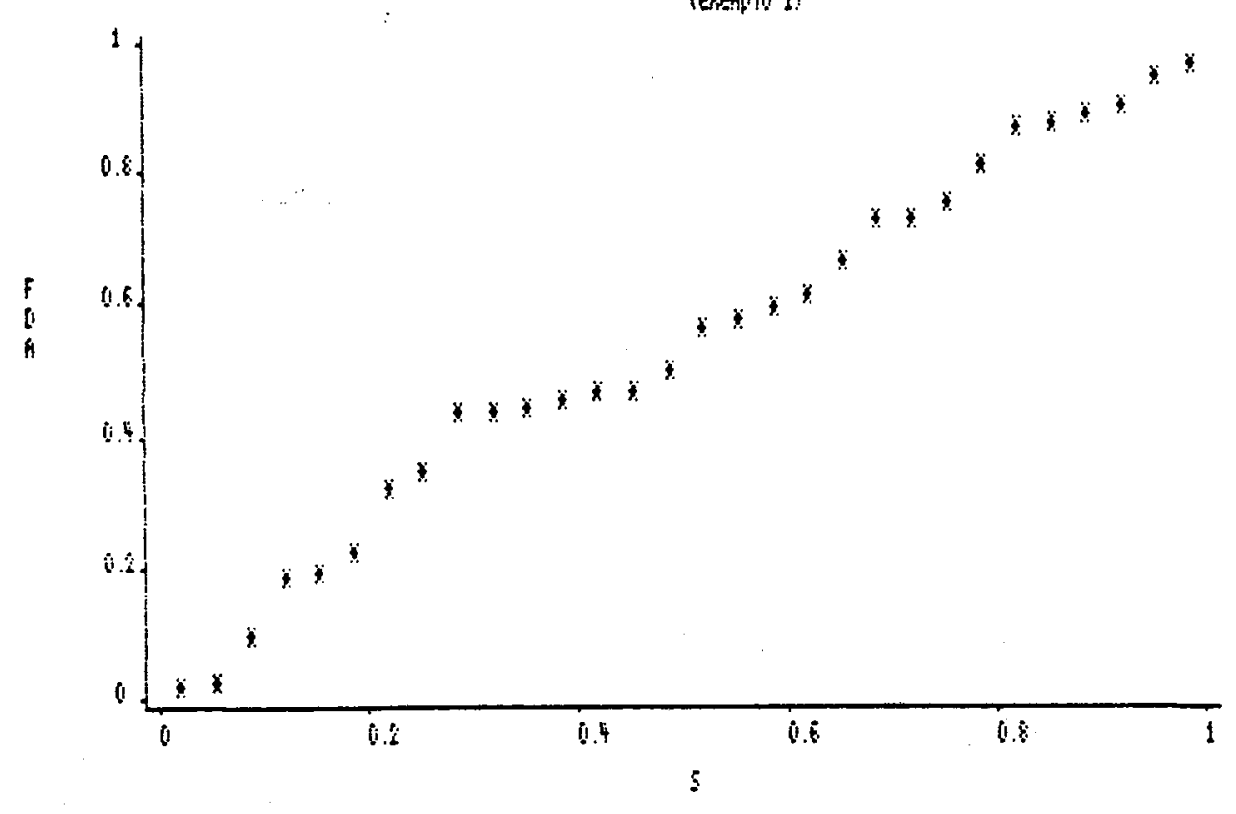


7.1 - análise do modelo Gaussiano Inverso sem variável estresse - pág. 79

\section{EXEMPLO 2}

TABELA 7.4

DISTRIBUIÇÕES DE FREQUÉNCIA EMPÍRICA E OBSERVADA

\begin{tabular}{|c|c|c|c|c|}
\hline i & $F(x)$ & $S(x)$ & $i / n$ & $(i-1) / n$ \\
\hline 1 & 0.005554 & 0.021739 & 0.043478 & 0.000000 \\
\hline 2 & 0.067433 & 0.065217 & 0.086957 & 0.043478 \\
\hline 3 & 0.109737 & 0.108696 & 0.130435 & 0.086957 \\
\hline 4 & 0.218549 & 0.152174 & 0.173913 & 0.130435 \\
\hline 5 & 0.226795 & 0.195652 & 0.217391 & 0.173913 \\
\hline 6 & 0.275159 & 0.239130 & 0.260870 & 0.217391 \\
\hline 7 & 0.315318 & 0.282609 & 0.304348 & 0.260870 \\
\hline 8 & 0.361589 & 0.326087 & 0.347826 & 0.304348 \\
\hline 9 & 0.363222 & 0.369565 & 0.391304 & 0.347826 \\
\hline 10 & 0.392322 & 0.413043 & 0.434783 & 0.391304 \\
\hline 11 & 0.411371 & 0.456522 & 0.478261 & 0.434783 \\
\hline 12 & 0.558458 & 0.500000 & 0.521739 & 0.478261 \\
\hline 13 & 0.567462 & 0.543478 & 0.565217 & 0.521739 \\
\hline 14 & 0.567462 & 0.586957 & 0.608696 & 0.565217 \\
\hline 15 & 0.570008 & 0.630435 & 0.652174 & 0.608696 \\
\hline 16 & 0.707857 & 0.673913 & 0.695652 & 0.652174 \\
\hline 17 & 0.769183 & 0.717391 & 0.739130 & 0.695652 \\
\hline 18 & 0.800562 & 0.760870 & 0.782609 & 0.739130 \\
\hline 19 & 0.832180 & 0.804348 & 0.826087 & 0.782609 \\
\hline 20 & 0.835377 & 0.847826 & 0.869565 & 0.826087 \\
\hline 21 & 0.908999 & 0.891304 & 0.913043 & 0.869565 \\
\hline 22 & 0.909293 & 0.934783 & 0.956522 & 0.913043 \\
\hline 23 & 0.973155 & 0.978261 & 1.000000 & 0.956522 \\
\hline
\end{tabular}

As estatisticas de K-S obtidas foram,

$$
\begin{aligned}
& \mathrm{D}_{o b s}^{K S+}=0.0822 \text { e } \mathrm{D}_{o b s}^{K S-}=0.0881, \text { portanto } \\
& \mathrm{D}_{\text {obs }}^{K S}=0.0822 \text { e } \phi=3.2073 .
\end{aligned}
$$

Assim, fixando $\gamma=0.05$ temos, $\mathrm{D}_{c r i}^{K S}=0.1851$, portanto $\mathrm{D}_{o b s}^{K S}<\mathrm{D}_{c r i}^{K S}$, indicando um bom ajuste do modelo Gaussiano inverso (ver gráfico 7.2). 
7.1 - análise do modelo Gaussiano Inverso sem variável estresse - pág. 80 GRÁFICO 7.2

GRAFICO PROBABILISTICO - DMPIRICO US. OBSERUADO (exetrio as

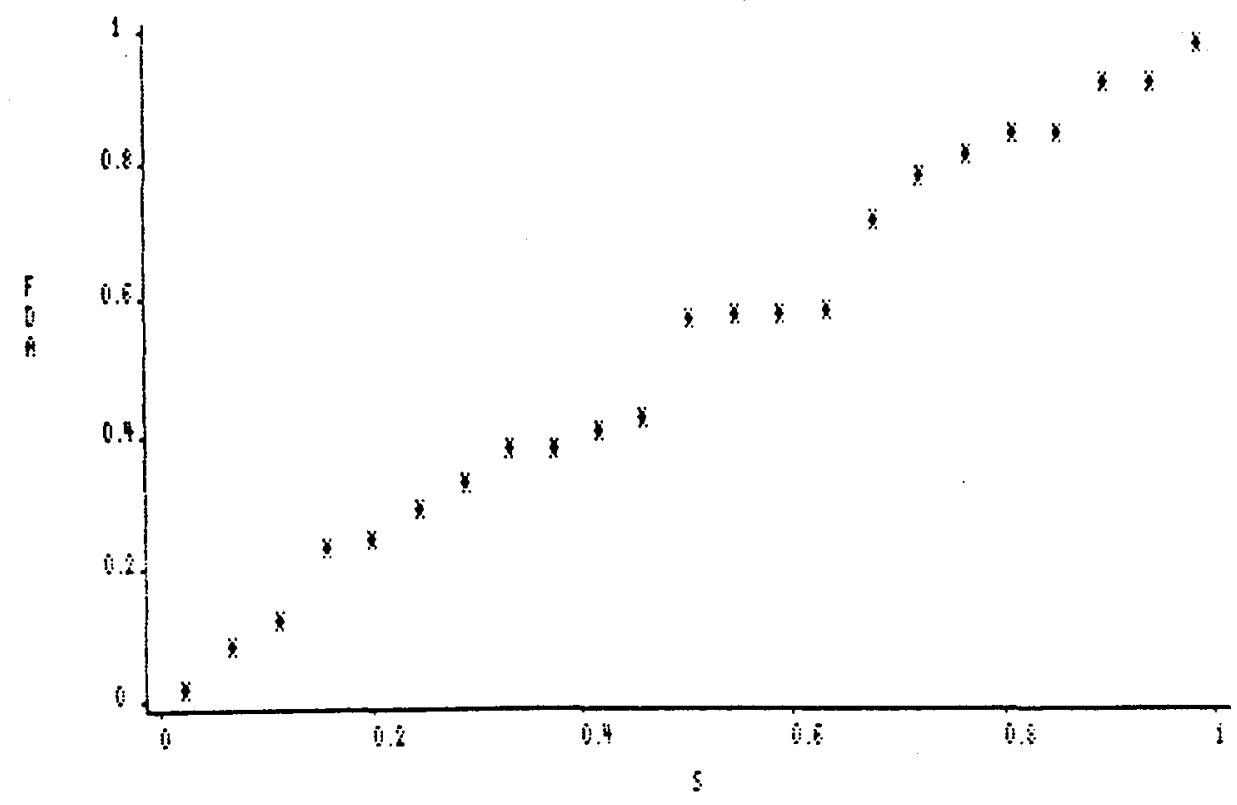

ANÁLISE BAYESIANA - PARAMETRIZAÇÃO $(0, \lambda)$.

Agora apresentamos uma análise Bayesiana para os 2 exemplos considerados:

\section{EXEMPLO 1}

\begin{tabular}{|l|l|}
\hline $\begin{array}{l}\text { Densidades a } \\
\text { Priori }\end{array}$ & \multicolumn{1}{|c|}{ Densidades a Posteriori } \\
\hline $\begin{array}{l}\text { Priori de } \\
\text { Jeffreys } \\
\begin{array}{l}(\theta \text { e } \lambda \\
\text { desconhecidos })\end{array}\end{array}$ & $\begin{array}{l}\pi(\theta / \text { dados }) \propto \theta^{-1.5}\left(31.11 \theta^{-2}-60 \theta^{-1}+39.26451\right)^{-15.5}, \\
\text { onde } \theta>0 . \text { A sua moda é dada por } \hat{\theta}=1.02(\text { gráfico } 7.3) . \\
\pi(\lambda / \text { dados }) \propto \lambda^{14} \exp \{-5.167455 \lambda\}, \\
\text { onde } \lambda>0 . \text { A sua moda é dada por } \hat{\lambda}=2.701 \text { (gráfico } 7.4) .\end{array}$ \\
\hline $\begin{array}{l}\text { Priori de } \\
\text { Jeffreys } \\
\text { (com } \lambda=2.701)\end{array}$ & $\begin{array}{l}\pi\left(\theta / \text { dados) } \propto \theta^{-1.5} \exp \left\{-\left(42.014055 \theta^{-2}-81.03 \theta^{-1}\right)\right\},\right. \\
\text { onde } \theta>0 \text { e a sua moda é dada por } \hat{\theta}=1.02 \text { (gráfico } 7.5) .\end{array}$ \\
\hline
\end{tabular}




\begin{tabular}{|c|c|}
\hline \multicolumn{2}{|r|}{ EXEMPLO 2} \\
\hline $\begin{array}{l}\text { Densidade } \\
\text { a Priori }\end{array}$ & Densidade a Posteriori \\
\hline $\begin{array}{l}\text { Priori de } \\
\text { Jeffreys } \\
(\theta \in \lambda \\
\text { desconhecidos) }\end{array}$ & $\begin{array}{l}\pi(\theta / \text { dados }) \propto \theta^{-1.5}\left(1661.16 \theta^{-2}-46 \theta^{-1}+0.417726\right)^{-12}, \\
\text { onde } \theta>0 . \text { A sua moda é dada por } \hat{\theta}=70.4(\text { gráfico } 7.6) \text {. } \\
\pi(\lambda / \text { dados }) \propto \lambda^{10.5} \exp \{-0.049634 \lambda\} \\
\text { onde } \lambda>0 . \text { A sua moda é dada por } \hat{\lambda}=212.00 \text { (gráfico } 7.7) \text {. }\end{array}$ \\
\hline $\begin{array}{l}\text { Priori de } \\
\text { Jeffreys } \\
(\operatorname{com} \lambda=212)\end{array}$ & $\begin{array}{l}\pi(\theta / \text { dados }) \propto \theta^{-1.5} \exp \left\{-\left(176082.9604 \theta^{-2}-4876.0 \theta^{-1}\right)\right\}, \\
\text { onde } \theta>0 \text { e a sua moda é dada por } \dot{\theta}=70.4 \text { (gráfico } 7.8) \text {. }\end{array}$ \\
\hline
\end{tabular}

\section{INTERVALOS DE CONFIANCCA E DE CREDIBILIDADE PARA OS PARĀMETROS}

Nas tabelas 7.5 e 7.6 temos os intervalos aproximados com $95 \%$ de confiança e de credibilidade para os parâmetros $\theta$ e $\lambda$ nos exemplos 1 e 2 .

\section{EXEMPLO 1}

\section{TABELA 7.5}

\begin{tabular}{|l|c|c|}
\hline Parâmetro & IC $\left(\mathrm{I}_{0}\right)$ & ICR \\
\hline$\theta$ & $(0.815207,1.258793)$ & $(0.810341,1.229659)$ \\
\hline$\lambda$ & $(1.433800,4.371771)$ & $(1.286156,4.115844)$ \\
\hline
\end{tabular}

Da tabela 7.5, verificamos resultados similares quanto a $\theta$ considerando o método Clássico e o método Bayesiano com uma densidade a priori não-informativa. Entretanto, com respeito a $\lambda$ observamos uma amplitude menor para o caso Bayesiano, indicando uma precisão melhor. 
7.J - análise do modelo Gaussiano Inverso sem variável estresse - pág. 82

\section{EXEMPLO 2}

TABELA 7.6

\begin{tabular}{|l|c|c|}
\hline Parámetro & IC $\left(\mathrm{I}_{0}\right)$ & ICR \\
\hline$\theta$ & $(55.743829,88.704867)$ & $(55.262984,85.537016)$ \\
\hline$\lambda$ & $(\mathbf{9 7 . 7 7 5 3 0 6 , 3 6 5 . 5 7 2 8 8 3 )}$ & $(\mathbf{8 3 . 7 7 0 0 9 9 , 3 4 0 . 2 2 9 9 0 1 )}$ \\
\hline
\end{tabular}

Da tabela 7.6, verificamos resultados similares considerando o método Clássico e o método Bayesiano com uma densidade a priori não-informativa.

Nos gráficos $7.3,7.4,7.5,7.6,7.7$ e 7.8 , temos as várias densidades a posteriori para os parámetros do modelo.

\section{GRÁFICO 7.3}

DENSIDADE A POSTERIORI MARGINAL PARA THETA (existio 1 )

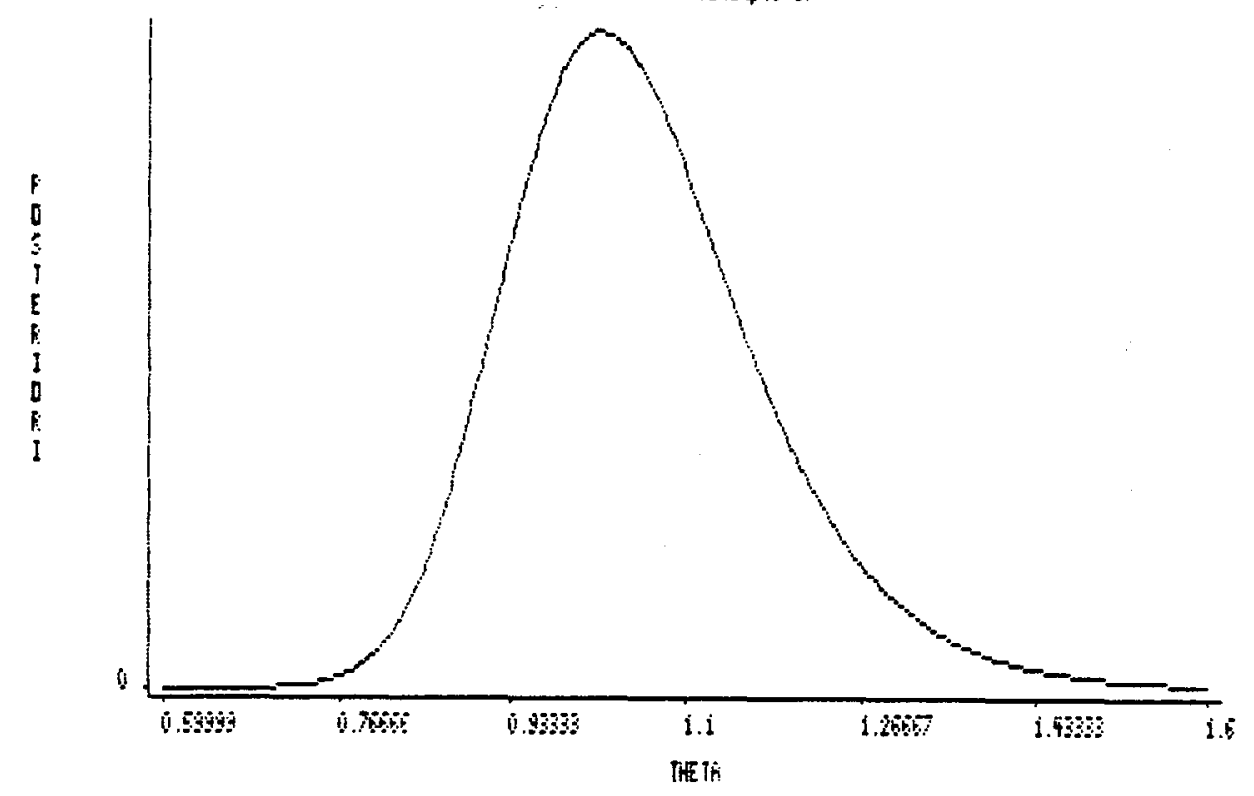


7.1 - análise do modelo Gaussiano Inverso sem variável estresse - pág. 89 GRÁFICO 7.4

DENSIDADE A POSTERIORI MARGINAL PARA LAMBDA (exentio l:

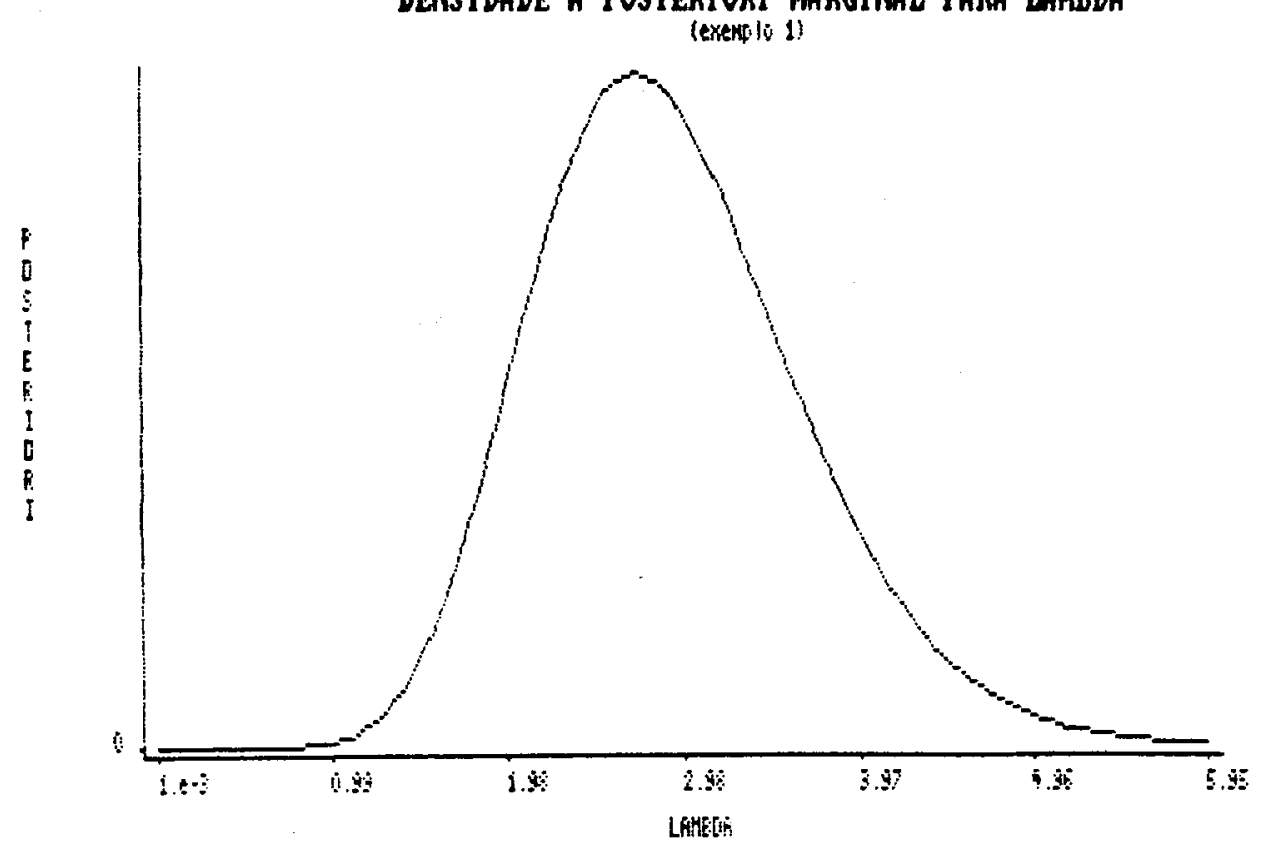

GRÁFICO 7.5

DENSIDADE a POSTERIORI MaRGINaL PaRá IHETa CON LAMBDa COHHECIDO

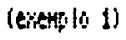

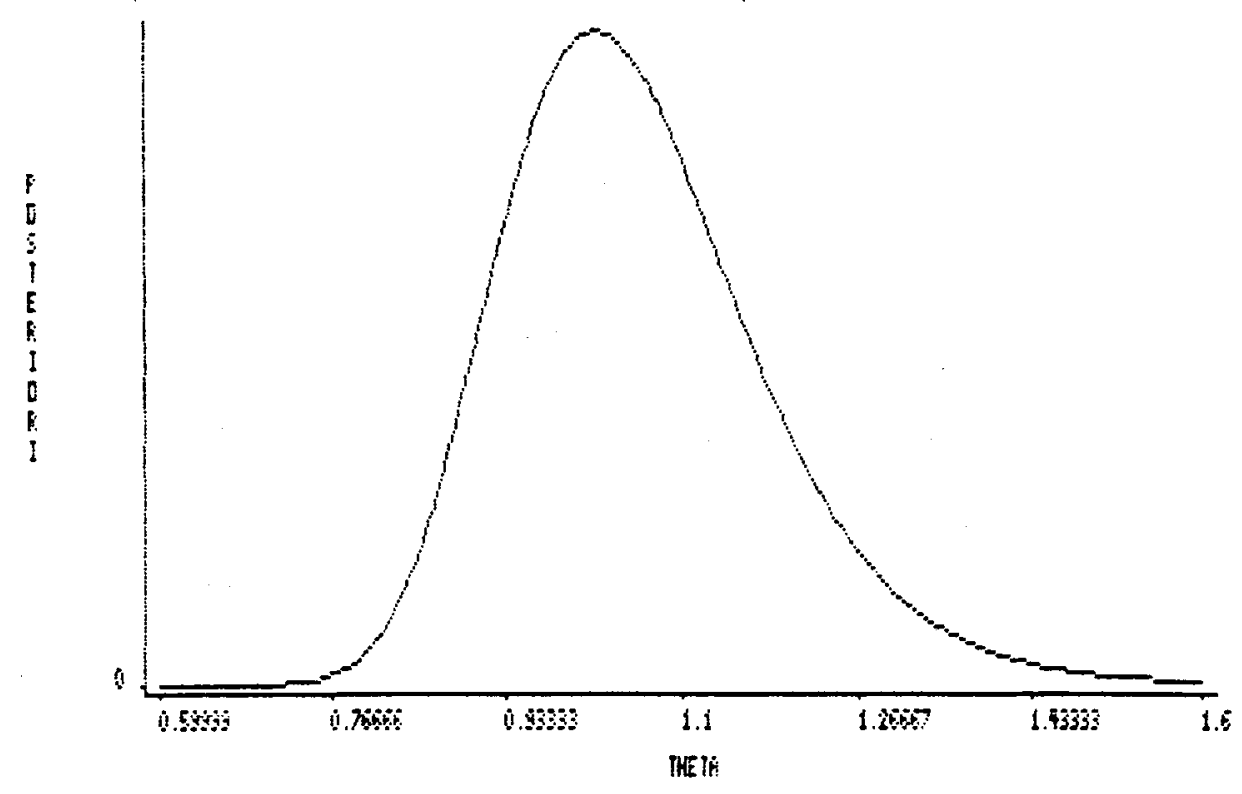


7.1 - análise do modelo Gaussiano Inverso sem variável estresse - pág. 84 GRÁFICO 7.6

DENSIDADE a POSTERIORI MARGINAL PARA IHETA (EYantis 2)

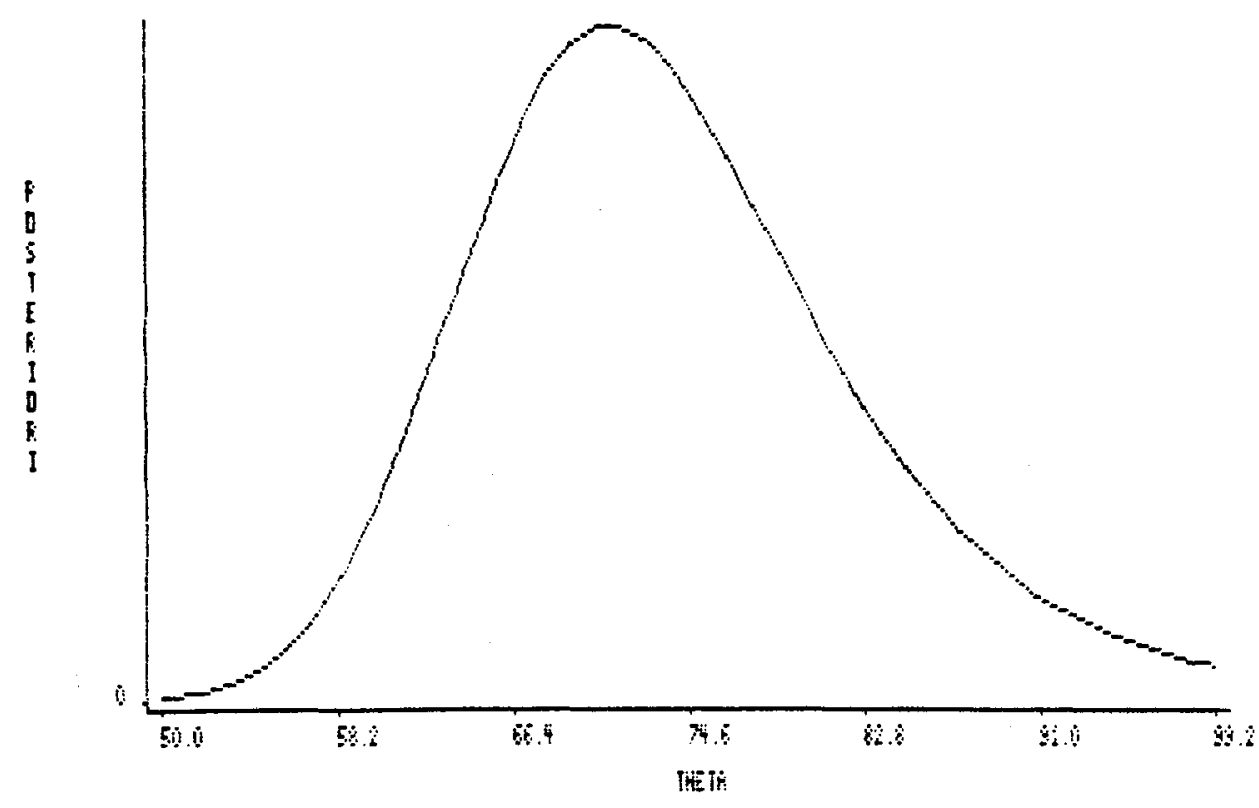

GRÁFICO 7.7

DENSIDADE A POSIERIORI MARGINAL PARA LAMBDA

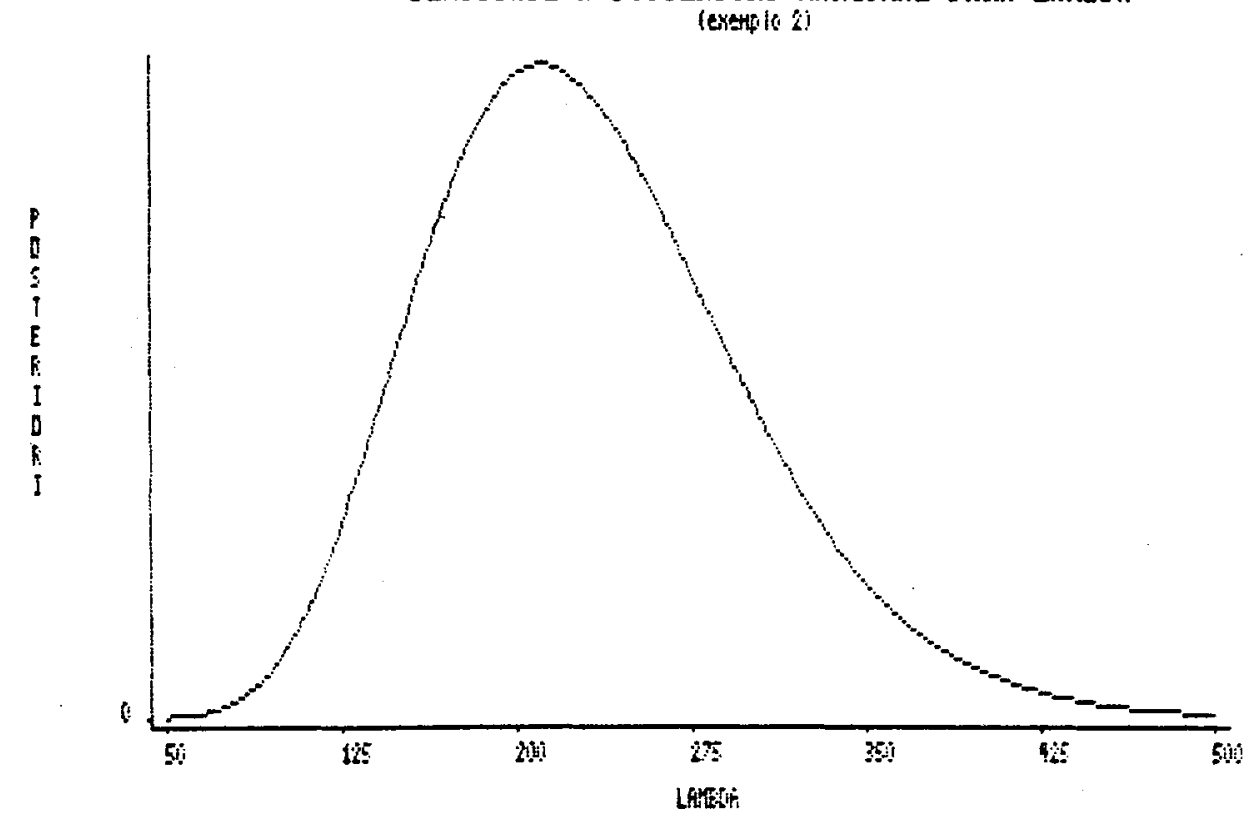


7.1 - análisc do modclo Gaussiano Invcrso sem variável estresse - pág. 85 GRÁFICO 7.8

DENSIDADE A POSIERIORI MARGINAL PARA IHETA COM LAMBDA CONHECIDO (axersit: 2 !

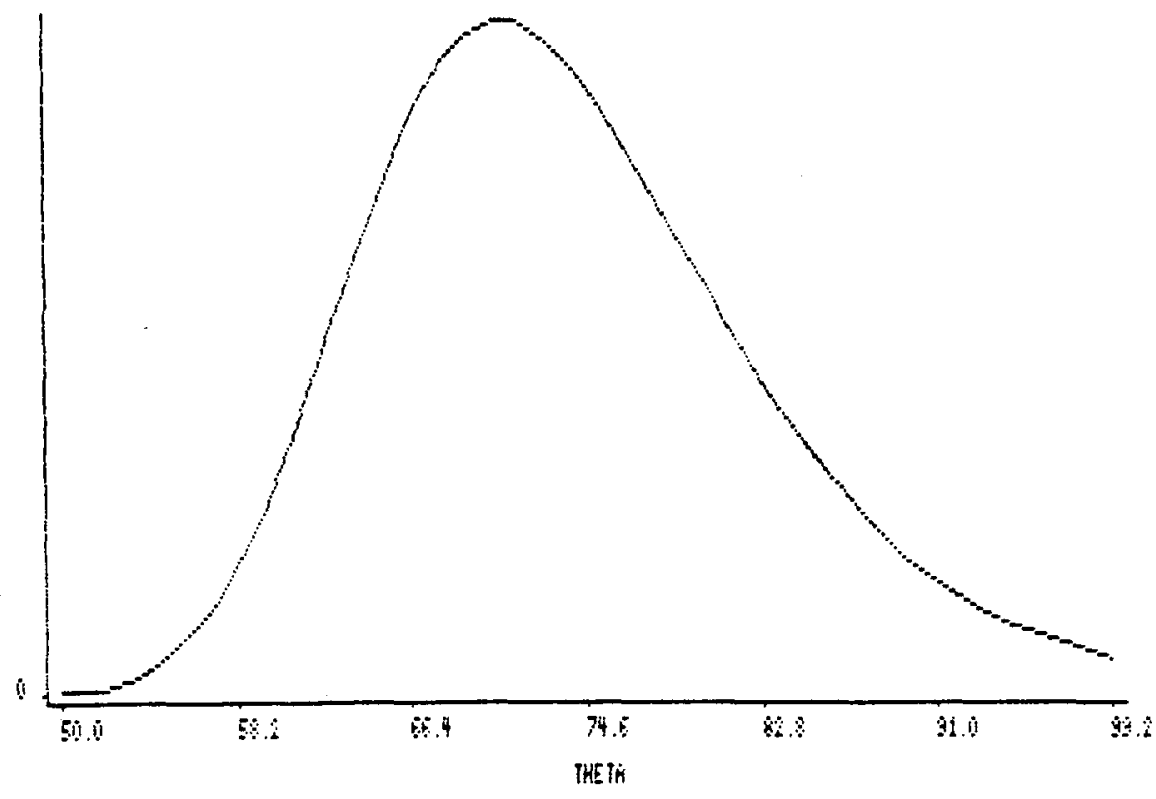

ANÁlISE CLÁSSICA - PARAMETRIZAÇÃO $(\mu=1 / \theta, \lambda)$.

Agora, refazemos a análise estatística considerando a parametrização $\mu=1 / \theta$ e $\lambda$.

EXEMPLO 1
$\mathrm{n}=\mathbf{3 0}$
$\mathrm{n}=23$
$\overline{\mathrm{Y}}=1.037$
$\mathrm{R}=1.308817$
$\overline{\mathrm{Y}}=72.224348$
$\mathrm{R}=0.018162$

\begin{tabular}{lll}
\hline Dados & $\mathrm{n}=30$ & $\mathrm{n}=23$ \\
$\overline{\mathrm{Y}}=1.037$ & $\overline{\mathrm{Y}}=72.224348$ \\
$\mathrm{R}=1.308817$ & $\mathrm{R}=0.018162$
\end{tabular}

EMV

$$
\hat{\mu}=0.96432
$$$$
\dot{\mu}=0.013846
$$$$
\hat{\lambda}=2.902786
$$$$
\bar{\lambda}=231.674094
$$

\begin{tabular}{|c|c|c|c|c|c|}
\hline $\begin{array}{l}\text { matrizes de } \\
\text { informação }\end{array}$ & & {$\left[\begin{array}{ll}90.30566 & 0\end{array}\right.$} & & {$[384847.738255$} & 0 \\
\hline observadas & $\mathrm{I}_{0}=$ & 1780160 & $I_{0}=$ & 0 & 0000214 \\
\hline
\end{tabular}


7.1 - análise do modelo Gaussiano Inverso sem variável estresse - pág. 86 $\mathrm{I}_{0}^{-1}=\left[\begin{array}{cc}0.011074 & 0 \\ 0 & 0.561744\end{array}\right]$

$\mathrm{I}_{0}^{-1}=\left[\begin{array}{cc}2.5984 \mathrm{e}-006 & 0 \\ 0 & 4667.20747\end{array}\right]$
variâncias
$\hat{\mathrm{V}}(\hat{\theta})=\mathbf{0 . 0 1 1 0 7 4}$
$\hat{\mathrm{V}}(\hat{\theta})=2.5984 \mathrm{e}-006$
estimadas
$\hat{\mathrm{V}}(\hat{\lambda})=0.561744$
$\hat{\mathrm{V}}(\hat{\lambda})=4667.20747$

resultados análogos foram encontrados usando a matriz de informaça de Fisher

ANÁLISE BAYESIANA - PARAMETRIZAÇÃO $(\mu=1 / \theta, \lambda)$.

Uma análise Bayesiana nesta parametrizaçāo é dada abaixo:

\begin{tabular}{|c|c|}
\hline \multicolumn{2}{|r|}{ EXEMPLO 1} \\
\hline $\begin{array}{l}\text { Densidade } \\
\text { a Priori }\end{array}$ & Densidade a Posteriori \\
\hline $\begin{array}{l}\text { Priori Não } \\
\text { Informativa } \\
\text { ( } \mu \text { e } \lambda \\
\text { desconhecidos) }\end{array}$ & $\begin{array}{l}\pi(\mu, \lambda / \text { dados })=k \lambda^{14} \exp \left\{-5.167449 \lambda\left[1+2.799215(1.037 \mu-1)^{2}\right]\right\} \\
\text { onde } \mu>0 \text { e } \lambda>0 \text { e } k \text { é a constante normalizadora. As modas desta densidade são, } \\
\tilde{\mu}=0.96432 \text { e } \bar{\lambda}=2.709267 \text {. (gráfico } 7.9) \text {. } \\
\pi(\mu / \text { dados })=(2.1203) \Gamma(15)(5.167449)^{-15}\left[1+3.010189(\mu-0.96432)^{2}\right] \\
\text { onde } \mu>0 . \text { A sua moda é dada por } \hat{\mu}=0.961 \text { (gráfico } 7.10) \text {. } \\
\pi(\lambda / \text { dados })=\Phi\left([28.929605 \lambda]^{1 / 2}\right) \frac{(5.167449 \lambda)^{14.5}}{\lambda \Gamma(14.5)} \exp \{-5.167449 \lambda\} \\
\text { onde } \lambda>0 . \text { A sua moda é dada por } \hat{\lambda}=2.601 \text { (gráfico } 7.11 \text { ). }\end{array}$ \\
\hline
\end{tabular}


7.1 - análise do modelo Gaussiano Inverso sem variável estresse - pág. 87

\begin{tabular}{|c|c|}
\hline \multicolumn{2}{|r|}{ EXEMPLO 2} \\
\hline $\begin{array}{l}\text { Densidade } \\
\text { a Priori }\end{array}$ & Densidade a Posteriori \\
\hline $\begin{array}{l}\text { Priori Não } \\
\text { Informativa } \\
\text { ( } \mu \text { e } \lambda \\
\text { desconhecidos) }\end{array}$ & $\begin{array}{l}\pi(\mu, \lambda / \text { dados })=k \lambda^{10.5} \exp \left\{-0.049639 \lambda\left[1+3.207701(72.224348 \mu-1)^{2}\right]\right\} \\
\text { onde } \mu>0 \text { e } \lambda>0 \text { e k é a constante normalizadora. As modas desta densidade são, } \\
\tilde{\mu}=0.013846 \text { e } \tilde{\lambda}=211.528521 \text { (gráfico } 7.12) \text {. } \\
\pi(\mu / \text { dados })=k \Gamma(11.5)(0.049639)^{-11.5}\left[1+16732.51 .38(\mu-0.013846)^{2}\right\} \\
\text { onde } \mu>0 \text { e } k \text { é valor próximo de zero. A sua moda é dada por } \hat{\mu}=0.0141 \text {. } \\
\text { (gráfico } 7.13) \text {. } \\
\pi\left(\lambda / \text { dados) }=\Phi\left([0.318452 \lambda]^{1 / 2}\right) \frac{(0.049639 \lambda)^{11}}{\lambda \Gamma(11)} \text { exp }\{-0.049639 \lambda\}\right. \\
\text { onde } \lambda>0 . \text { A sua moda é dada por } \hat{\lambda}=201.2 \text { (gráfico } 7.14) \text {. }\end{array}$ \\
\hline
\end{tabular}

\section{INTERVALOS DE CONFLANÇA E CREDIBILIDADE PARA OS PARĀMETROS}

Nas tabelas 7.7 e 7.8 temos intervalos aproximados com $95 \%$ de confiança e credibilidade para $\mu$ e $\lambda$.

\section{EXEMPLO 1}

\section{TABELA 7.7}

\begin{tabular}{|l|c|c|}
\hline Parámetro & IC $\left(l_{0}\right)$ & ICR \\
\hline$\mu$ & $(0.758072,1.170569)$ & $(0.835553,1.093087)$ \\
\hline$\lambda$ & $(1.433800,4.371771)$ & $(1.552707,4.424067)$ \\
\hline
\end{tabular}

Utilizando a aproximação normal, o ICR de $95 \%$ (ver 2.78) para $\mu$ é dado por, (0.754741, 1.167259$)$. 


\section{EXEMPLO 2}

\section{TABELA 7.8}

\begin{tabular}{|l|l|l|}
\hline Parámetro & IC $\left(\mathrm{I}_{0}\right)$ & ICR \\
\hline$\mu$ & $(0.010686,0.017005)$ & $(0.012119,0.015573)$ \\
\hline$\lambda$ & $(97.775306,365.572883)$ & $(161.638730,460.550832)$ \\
\hline
\end{tabular}

Utilizando a aproximação normal, o ICR de $95 \%$ (ver 2.78 ) para $\mu$ é dado por, $(0.010935,0.017265)$.

Nos gráficos $7.9,7.10,7.11,7.12,7.13$ e 7.14 temos as várias densidades a posteriori obtidas para os 2 exemplos.

\section{$\underline{\text { GRÁFICOO } 7.9}$}

\section{CONTORNOS DA POSTERIORI CONJUKTA MUL E LAKBDA}

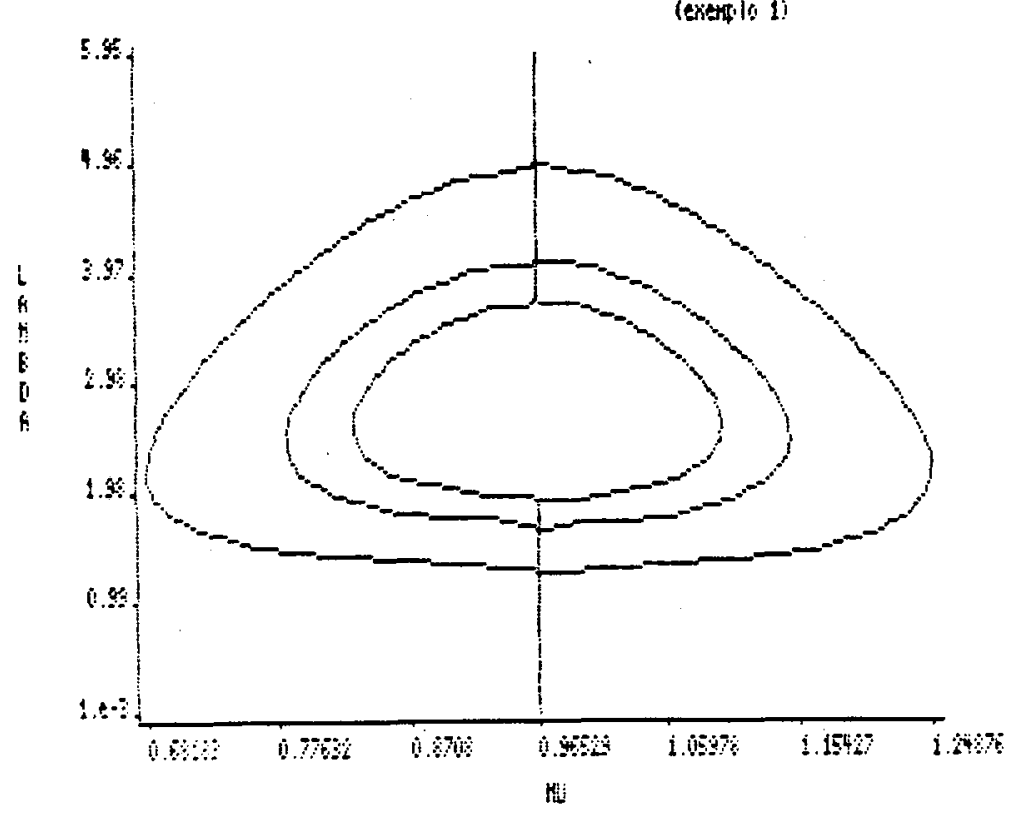

Legenda:

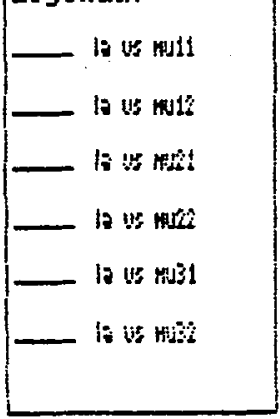


7.1 - análise do modelo Gaussiano Inverso sem variável estresse - pág. 89 GRÁFICO 7.10

DENSIDADE A POSTERIORI MARGINAL PARA NU

(EKetrilo 1 )

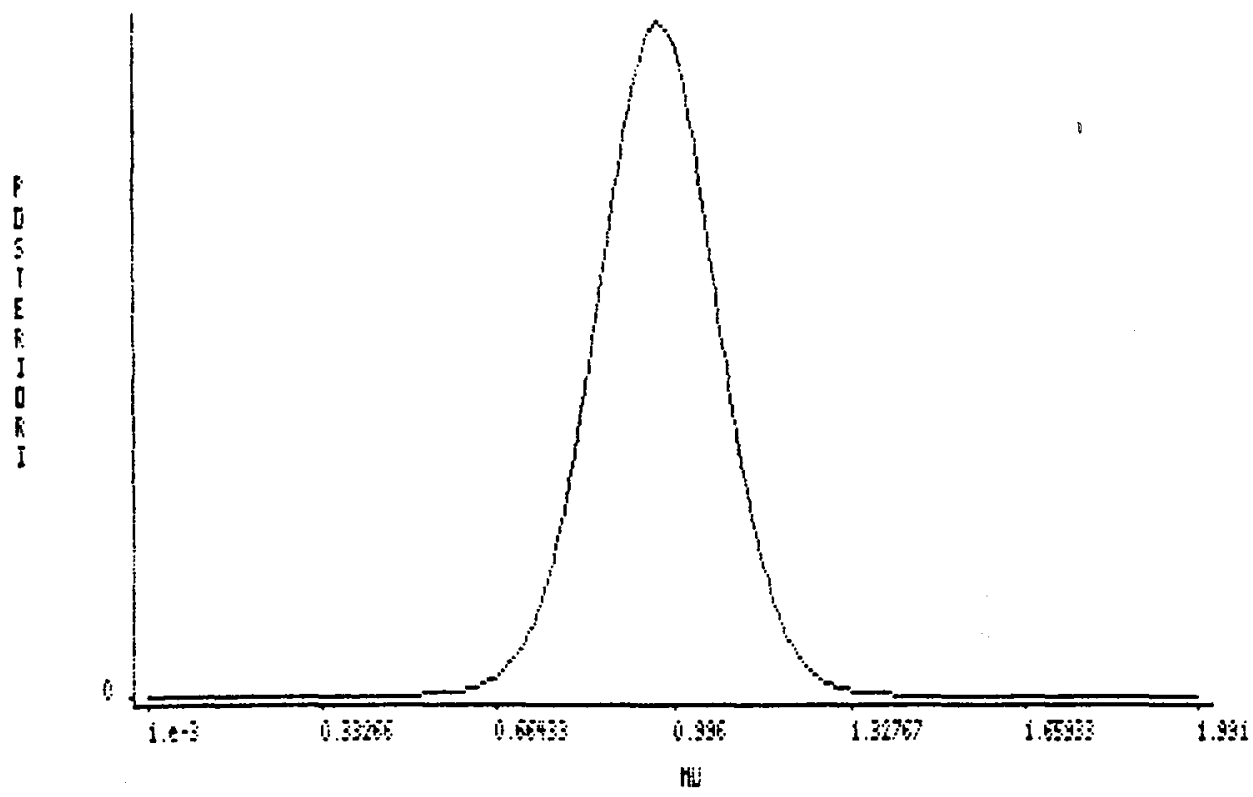

GRÁFICO 7.11

DENSIDADE A POSTERIORI MARGINAL PARA LAMBDA (t)istis

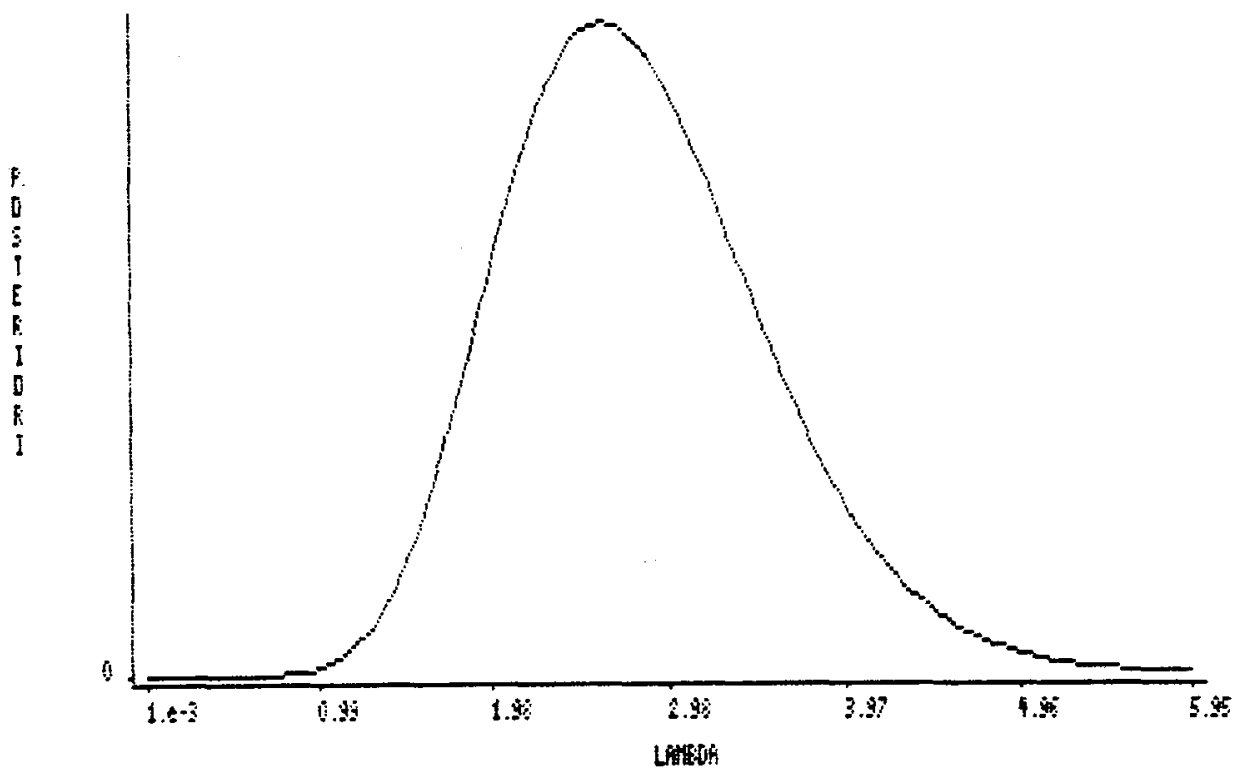


7.1 - análise do modelo Gaussiano Inverso sem variávcl estresse - pág. 90 GRÁFICO 7.12

\section{CONTORNOS PA POSTERIORI CONJUNTA MU E LAMBDA}

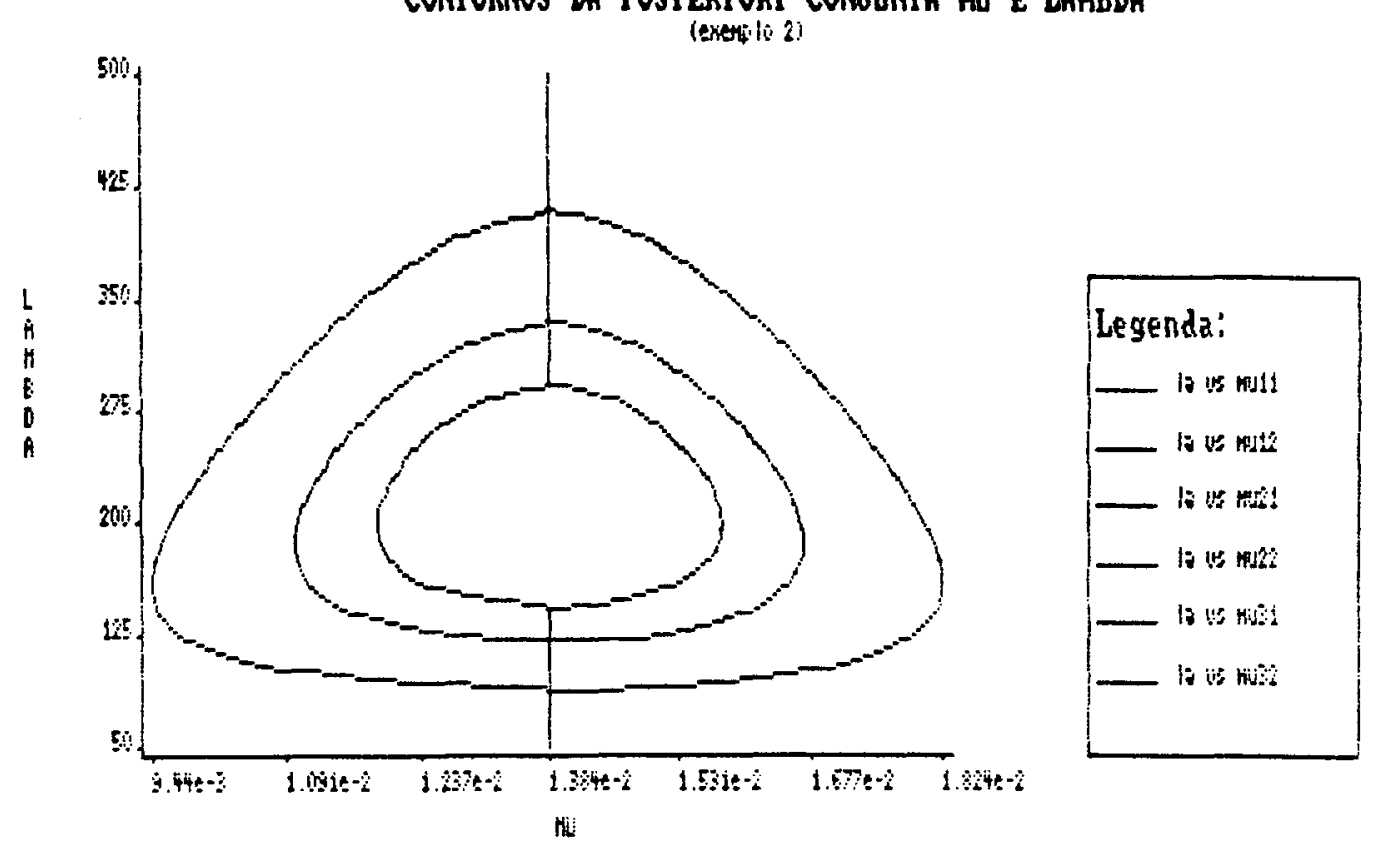

GRÁFICO 7.13

DENSIDAdE a POSTERIORI NaRCINAL PARA MUI

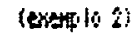

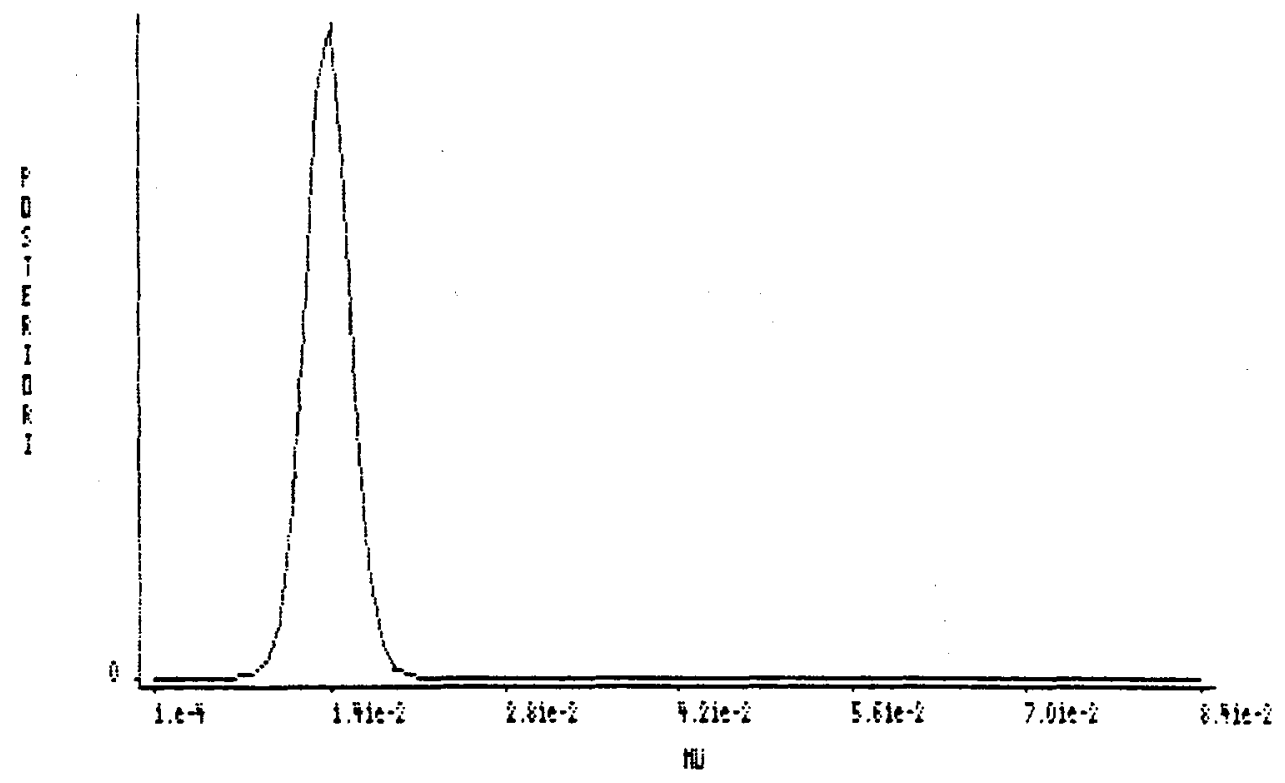


7.2 análise do modelo de regressào linear inverso - análise clássica e bayesiana - pág. 91 GRÁFICO 7.14

DENSIDADE A POSTERIORI MARGINAL PARA LAMBDA

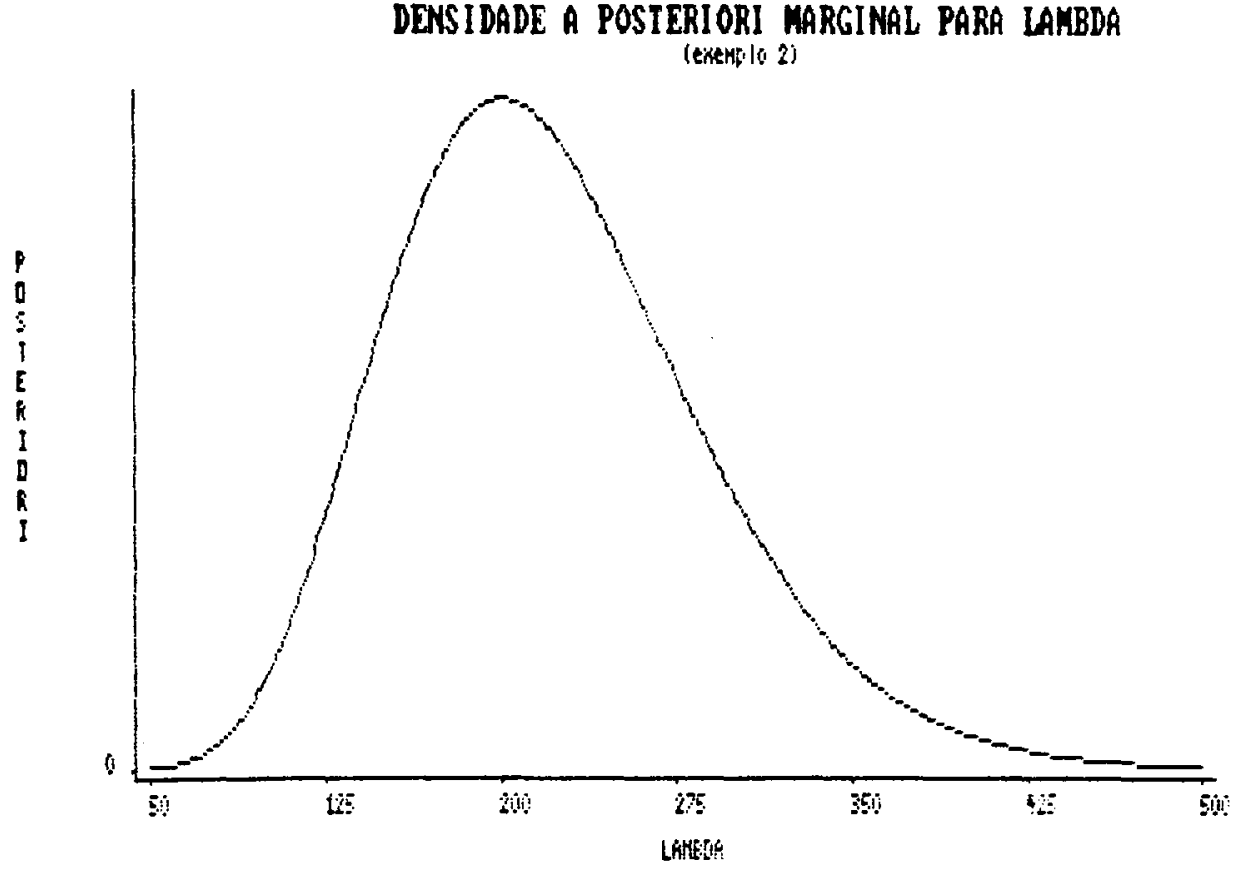

Concluimos, que os resultados Bayesianos são análogos aos resultados clássicos, quando usamos densidades a priori não-informativa, mas são de grande simplicidade, e pode ser uma grande vantagem quando utilizamos com dados. Além disso, poderiamos ter melhores inferências quando os pesquisadores tem opiniões a priori sobre os parâmetros, pois os resultados aproximados seriam utilizados da mesma forma.

\subsection{ANÁLISE DO MODELO DE REGRESSÃO LINEAR INVERSO-ANÁLISE CLÁSSICA E BAYESIANA.}

\subsubsection{TERCEIRO EXEMPLO}

A seguir apresentamos um conjunto de dados introduzidos por Nelson (1971). Este conjunto de dados é dado na tabela 7.9 e consiste dos tempos de falha de uma nova classe $\mathrm{H}$ de um material de isolação em prova num teste de motores em temperaturas elevadas de 190, 220, 240, e $260^{\circ} \mathrm{C}$. O tempo para a primeira falha é dado para cada unidade testada. Para cada temperatura, 10 unidades foram colocadas em teste e periodicamente examinadas para falha de isolação. $O$ tempo de falha dada na tabela é o tempo medio entre o tempo de inspeção quando a primeira falha foi encontrada no motor e o tempo de inspeção previa. 
7.2 onálise do modelo de regressão linear inverso - análise clássica e bayesiana - pág. 92

O objetivo original do experimento é determinar o tempo médio de falha numa temperatura, designada de $180{ }^{\circ} \mathrm{C}$ excede um tempo mínimo especifico. Os dados a $260{ }^{\circ} \mathrm{C}$ foram tomados de um lote de isolação diferente do lote usado nas outras temperaturas. Portanto, é importante investigar se os dados dos dois lotes foram consistentes. Nelson (1971), estabelece a inconsistencia dos dados a $260^{\circ} \mathrm{C}$ empregando técnicas gráficas e analíticas.

TABELA 7.9

TEMPOS DE FALHA EM UM TESTE DE VIDA ACELERADO DE UMA NOVA CLASSE H DE ISOLAÇÃO EM MOTORES

\begin{tabular}{|l|l|l|l|}
\hline \multicolumn{4}{|c|}{ Tempos de Falha (em horas) } \\
\hline $190{ }^{\circ} \mathrm{C}$ & $220{ }^{\circ} \mathrm{C}$ & $240^{\circ} \mathrm{C}$ & $260^{\circ} \mathrm{C}$ \\
\hline 7228 & 1764 & 1175 & 600 \\
7228 & 2436 & 1521 & 744 \\
7228 & 2436 & 1521 & 744 \\
8448 & 2436 & 1569 & 744 \\
9167 & 2436 & 1617 & 912 \\
9167 & 2436 & 1665 & 1128 \\
9167 & 3108 & 1665 & 1320 \\
9167 & 3108 & 1713 & 1464 \\
10511 & 3108 & 1761 & 1608 \\
10511 & 3108 & 1953 & 1896 \\
\hline
\end{tabular}

Para nosso propósito, consideramos um modelo de regressāo IG para esses dados excluindo a temperatura de $260^{\circ}$. C. A suposição de uma constante $\lambda$ é válida desde que os materiais nos primeiros três niveis sejam conhecidas como tendo uma origem comum. Na ausência de um modelo que relaciona a vida media de falha $(\theta)$ e a temperatura $(T)$, baseamos nosso modelo a partir das seguintes consideraçōes:

i) a regressão de $\bar{Y}_{i}^{-1}$ em $T_{i}^{3}$ leva a um valor $R_{r}^{2}=99.9 \%$, onde $R_{r}^{2}$ mede o ajuste do modelo,

ii) a variância amostral elevada a uma potência de $-\frac{1}{3}$ é aproximadamente linear em $T_{i}{ }^{3}$, e

iii) a transformação $T_{i}{ }^{3}-\left(180^{3}\right)$ é conveniente e produz EMV positivos. Sem uma translação de $\mathrm{T}_{i}^{3}$, a restrição $\alpha \geq 0$ imposta na definição de $\Omega$ não poderia ser verificada. 
7.2 análise do modelo de regressão linear inverso - análise clássica e bayesiana - pág. 99

Com essas consideraçōes, temos a distribuição do tempo de falha como uma densidade $\operatorname{IG}(\theta, \lambda) \operatorname{com} \theta^{-1}=\alpha+\beta \mathrm{x}$ onde $\mathrm{x}=10^{-8}\left[\mathrm{~T}^{3}-180^{3}\right]$.

Os dados a serem analizados foram transformados e sấ dados na tabela 7.10, onde a primeira coluna é denotada por $\mathrm{Y}=$ horas de falha/1000, e a segunda coluna denotada por $X=10^{-8}$ | $\left.\mathrm{T}^{3}-180^{3}\right]$.

TABELA 7.10

DADOS TRANSFORMADOS

\begin{tabular}{lcc}
\hline obs. & $\mathrm{Y}$ & $\mathrm{X}$ \\
\hline 1 & 7.2280 & 0.0103 \\
2 & 7.2280 & 0.0103 \\
3 & 7.2280 & 0.0103 \\
4 & 8.4480 & 0.0103 \\
5 & 9.1670 & 0.0103 \\
6 & 9.1670 & 0.0103 \\
7 & 9.1670 & 0.0103 \\
8 & 9.1670 & 0.0103 \\
9 & 10.511 & 0.0103 \\
10 & 10.511 & 0.0103 \\
11 & 1.7640 & 0.0482 \\
12 & 2.4360 & 0.0482 \\
13 & 2.4360 & 0.0482 \\
14 & 2.4360 & 0.0482 \\
15 & 2.4360 & 0.0482 \\
16 & 2.4360 & 0.0482 \\
17 & 3.1080 & 0.0482 \\
18 & 3.1080 & 0.0482 \\
19 & 3.1080 & 0.0482 \\
20 & 3.1080 & 0.0482 \\
21 & 1.1750 & 0.0799 \\
22 & 1.1750 & 0.0799 \\
23 & 1.5210 & 0.0799 \\
24 & 1.5690 & 0.0799 \\
25 & 1.6170 & 0.0799 \\
26 & 1.6650 & 0.0799 \\
\hline & & \\
\hline & &
\end{tabular}


7.2 análise do modelo de regressão linear inverso - análise clássica e bayesiana - pág. 94 continuaçāo da tabela 7.10

\begin{tabular}{ccc}
\hline obs. & $Y$ & $X$ \\
\hline 27 & 1.6650 & 0.0799 \\
28 & 1.7130 & 0.0799 \\
29 & 1.7610 & 0.0799 \\
30 & 1.9530 & 0.0799 \\
31 & 0.6000 & 0.1174 \\
32 & 0.7440 & 0.1174 \\
33 & 0.7440 & 0.1174 \\
34 & 0.7440 & 0.1174 \\
35 & 0.9120 & 0.1174 \\
36 & 1.1228 & 0.1174 \\
37 & 1.3200 & 0.1174 \\
38 & 1.4640 & 0.1174 \\
39 & 1.6080 & 0.1174 \\
40 & 1.8960 & 0.1174 \\
\hline
\end{tabular}

\section{ANÁLISE CLASSICA.}

Da tabela 7.10 e excluindo os dados a $260^{\circ} \mathrm{C}$, temos $\mathrm{n}=30$ e as seguintes estatisticas básicas (usando a matriz de informação observada) dadas por,

$$
\begin{aligned}
& V_{0}=4.333733 \\
& V_{1}=0.114535 \\
& v_{2}=0.005715 \\
& \bar{X}=0.046117 \\
& R=0.384891 \\
& D=0.011648
\end{aligned}
$$

e as seguintes estatisticas básicas (usando a matriz de informação de Fisher) dadas por,

$$
\begin{aligned}
& W_{0}=4.356187 \\
& W_{1}=0.114421 \\
& W_{2}=0.005715 \\
& \bar{X}=0.046117 \\
& R=0.384891 \\
& E=0.0011805
\end{aligned}
$$


7.2 análise do modelo de regressâo linear inverso - análise clássica e bayesiana - pág. 95 Assim, a matriz de informação observada de (3.25) $\dot{e}$,

$$
I_{0}=\left[\begin{array}{ccc}
13088.583972 & 345.914947 & 0 \\
345.914947 & 17.259857 & 0 \\
0 & 0 & 0.00148
\end{array}\right]
$$

e a inversa da matriz de informação observada $\mathrm{I}_{0}^{-1}$ de (3.27) é,

$$
\mathrm{I}_{0}^{-1}=\left[\begin{array}{ccc}
0.000162 & -0.003256 & 0 \\
-0.003256 & 0.123187 & 0 \\
0 & 0 & 675.658415
\end{array}\right]
$$

A matriz de informaçâo de Fisher $I(\psi)$ de (3.33) é,

$$
\mathrm{I}(\psi)=\left[\begin{array}{ccc}
13156.398635 & 345.57088 & 0 \\
345.57088 & 17.261602 & 0 \\
0 & 0 & 0.00148
\end{array}\right] \text {, }
$$

e a inversa da matriz de informação de Fisher $I^{-1}(\psi)$ de (3.34) é,

$$
\mathrm{I}^{-1}(\psi)=\left[\begin{array}{ccc}
0.00016 & -0.003209 & 0 \\
-0.003209 & 0.122179 & 0 \\
0 & 0 & 675.658415
\end{array}\right]
$$

Assumindo o modelo (3.1) onde $\mathrm{x}=10^{-8}\left[\mathrm{~T}^{3}-180^{3}\right]$ e uma distribuição Gaussiana Inversa para $\mathrm{Y}=$ Horas de falha/1000, os EMV para $\alpha, \beta, \lambda, \lambda^{-1}$ e $\theta_{1}$, e seus erros padröes (EP) obtidos pelas distribuições limitantes dos EMV são dados na seguinte tabela: 
7.2 análise do modelo de regressão linear inverso - análise clássica e bayesiana - pág. 96 TABELA 7.11

EMV PARA OS PARĀMETROS E SEUS RESPECTIVOS ERROS PADRÕES

\begin{tabular}{|l|l|l|l|}
\hline Parâmetro & EMV & EP $\left(\mathrm{I}_{0}\right)$ & EP $(\mathrm{I}(\psi))$ \\
\hline$\alpha$ & 0.0371 & 0.0127 & 0.0127 \\
$\beta$ & 7.3248 & 0.3510 & 0.3495 \\
$\lambda$ & 100.6721 & 25.9934 & 25.9934 \\
$\lambda^{-1}$ & 0.0099 & 0.0026 & 0.0026 \\
\hline
\end{tabular}

Os EMV dos tempos medios de falha $\theta$ e seus inversos $\theta^{-1}$ (em milhares de horas) em $190^{\circ} \mathrm{C}, 220^{\circ} \mathrm{C}$, e $240^{\circ} \mathrm{C}$ com seus erros padróes associados (EP) são dados na tabela a seguir:

TABELA 7.12

EMV PARA OS TEMPOS MÉDIOS DE FALHA E SEUS INVERSOS

EM TRESS NÍVEIS DE ESTRESSE ESPECIFICADO COM

SEUS RESPETIVOS ERROS PADRÕES

\begin{tabular}{|l|l|r|c|c|}
\hline \multirow{2}{*}{$\begin{array}{l}\text { Niveis } \\
\text { Estresse }\end{array}$} & \multicolumn{2}{|c|}{ EMV } & \multicolumn{2}{c|}{ Erros Padrōes } \\
\cline { 2 - 5 } & $\theta$ & $\theta^{-1}$ & $\operatorname{EP}\left(\mathrm{I}_{0}\right)$ & $\operatorname{EP}(\mathrm{I}(\psi))$ \\
\hline 190 & 8.897689 & 0.112389 & 0.01042 & 0.010357 \\
220 & 2.5646 & 0.389924 & 0.011601 & 0.011601 \\
240 & 1.606273 & 0.622559 & 0.020709 & 0.020682 \\
\hline
\end{tabular}

\section{ANÁLISE BAYESIANA.}

Nas seções 4.1 e 4.2 encontramos expressões para as densidades a posteriores marginais para os parâmetros de interesse. Os dados utilizados fornecem os valores:

$$
\begin{aligned}
& \mathrm{n}=30 \\
& \mathrm{n}_{i}=10 \\
& \mathrm{~V}_{0}=4.333733 \\
& \mathrm{~V}_{1}=0.114535 \\
& \mathrm{~V}_{2}=0.005715 \\
& R=0.38489
\end{aligned}
$$


7.2 análise do modelo de regressĩo linear inverso - análise clássica e bayesiana - pág. 97

$$
\begin{aligned}
& D=0.011648 \\
& \bar{X}=0.046117 \\
& x_{1}=0.0103 \\
& x_{2}=0.0482 \\
& x_{3}=0.0799
\end{aligned}
$$

e $\lambda=94$ para a análise quando assumimos que o parámetro é conhecido.

A densidade a posteriori para $\alpha$ de (4.12) é dada por,

$$
\pi(\alpha / \text { dados }) \propto\left\{W_{0}(\alpha, \hat{\beta}) W_{2}(\alpha, \hat{\beta})-W_{1}^{2}(\alpha, \hat{\beta})\right\}^{1 / 2}\left\{0.011648 \alpha^{2}-0.000866 \alpha+0.000073\right\}^{-15.5}
$$

onde $\alpha>0$ e $\hat{\beta}=\frac{\bar{X}-\alpha V_{1}}{V_{2}}, \operatorname{com} W_{l}(\alpha, \dot{\beta})=n^{-1} \sum_{i=1}^{3} n_{i} x_{i}^{l}\left(\alpha+\hat{\beta}_{x_{i}}\right)^{-1}, \quad l=0,1,2$.

A moda desta densidade a posteriori é $\hat{a}=0.0371$. No gráfico 7.15 mostramos a densidade a posteriori marginal para $\alpha$.

\section{GRÁFICO 7.15}

DENSIDADE a POSTERIORI MaRgINAL PARA aLFa

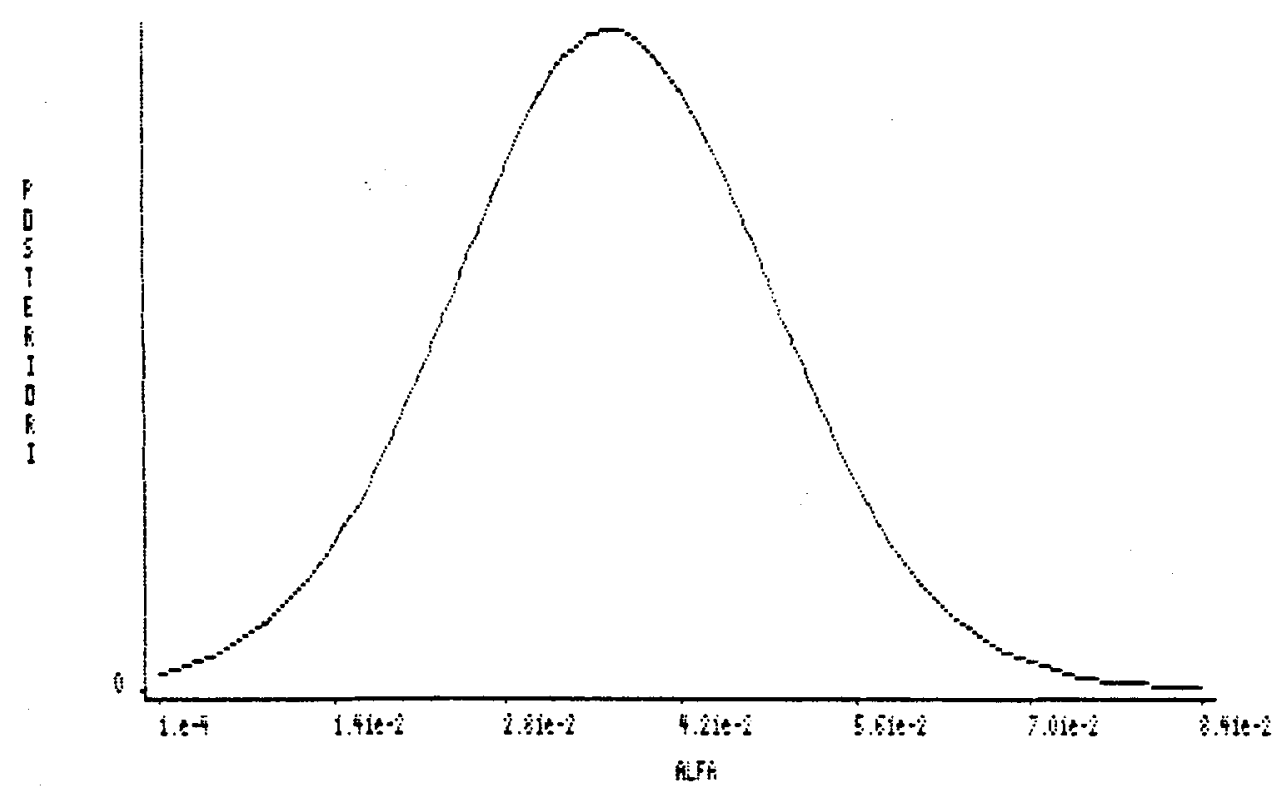


7.2 análise do modelo de regressão lincar inverso - análise clássica e bayesiana - pág. 98 A densidade a posteriori para $\beta$ de (4.13) é dada por,

$$
\pi(\beta / \text { dados }) \propto\left\{\mathrm{W}_{0}(\dot{a}, \beta) \mathrm{W}_{2}(\dot{\alpha}, \beta)-\mathrm{W}_{1}^{2}(\dot{a}, \beta)\right\}^{1 / 2}\left\{0.011648 \beta^{2}-0.170648 \alpha+0.668015\right\}^{-15.5}
$$

onde $\beta>0$ e $\hat{\alpha}=\frac{1-\beta V_{1}}{V_{0}}$, con $W_{l}(\hat{a}, \beta)=n^{-1} \sum_{i=1}^{3} n_{i} x_{i}^{l}\left(\hat{\alpha}+\beta x_{i}\right)^{-1}, \quad l=0,1,2$.

A moda desta densidade a posteriori é $\hat{\beta}=7.35$. No gráfico 7.16 mostramos a densidade a posteriori marginal para $\beta$.

\section{GRÁFICO 7.16}

DENSIDADE A POSTERIORI MARGINAL PARA BETA

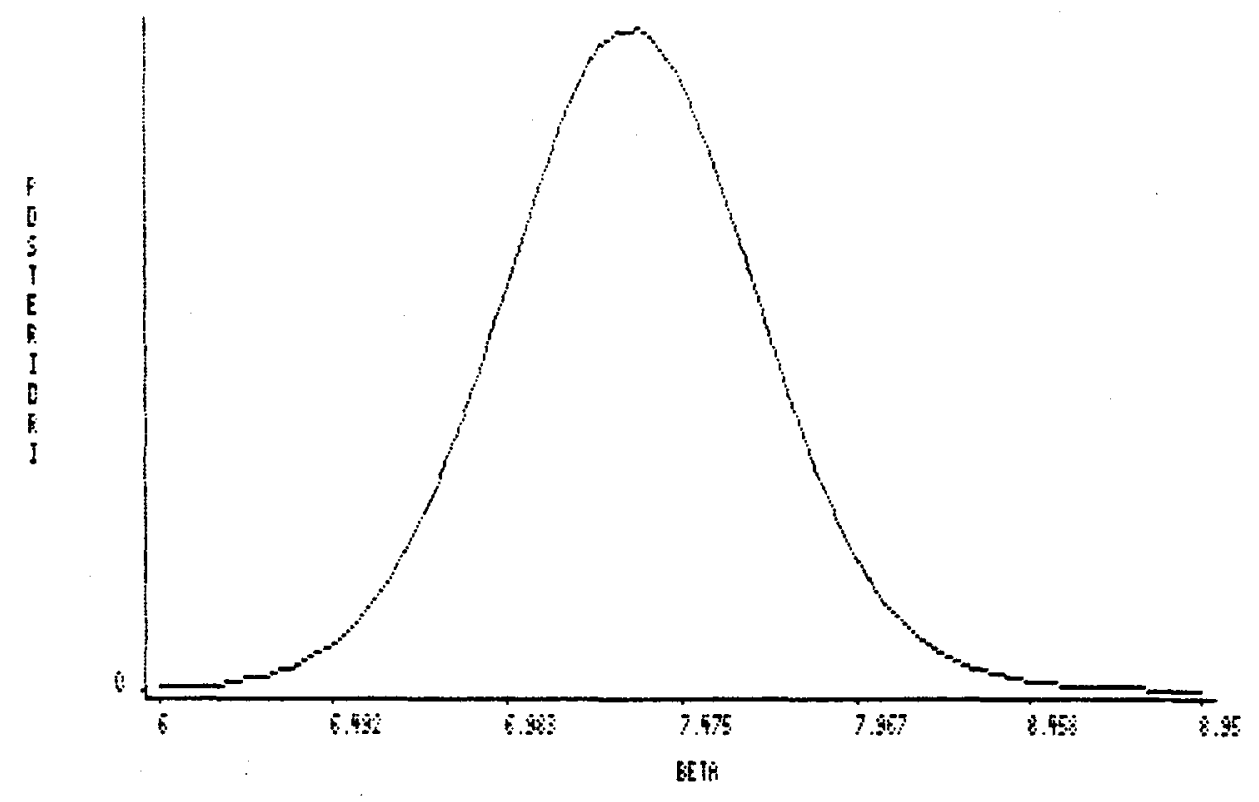

A densidade a posteriori marginal para $\lambda$ de (4.16) é dada por,

$$
\pi(\lambda / \text { dados }) \propto \lambda^{14} \exp \{-0.148538 \lambda\}
$$

onde $\lambda>0$.

A moda desta densidade a posteriori è $\dot{\lambda}=94$. No gráfico 7.17 mostramos a densidade a posteriori marginal para $\lambda$. 
7.2 análise do modelo de regressīo linear inverso - análise clássica e bayesiana - pág. 99 GRÁFICO 7.17

DENSIDADE A POSTERIORI MARGINAL PARA LAKBDA

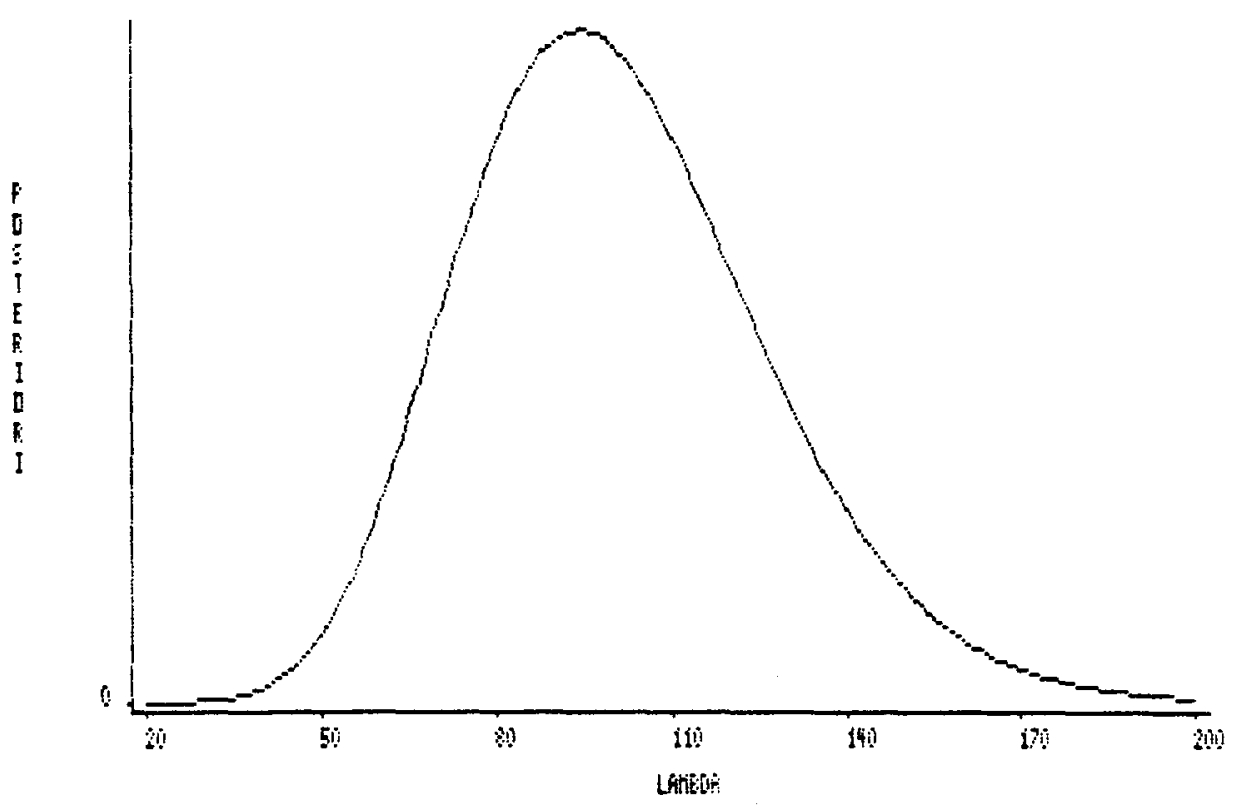

A densidade a posteriori marginal para $\theta_{1}$ de (4.23) é dada por, $\pi\left(\theta_{1} /\right.$ dados $) \propto \theta_{1}^{-2}\left\{W_{0}\left(\dot{\alpha}, \theta_{1}\right) W_{2}\left(\hat{\alpha}, \theta_{1}\right)-W_{1}^{2}\left(\hat{\alpha}, \theta_{1}\right)\right\}^{1 / 2}\left\{4315.594004 a_{3}\left(\theta_{1}\right)-a_{2}^{2}\left(\theta_{1}\right)\right\}^{-15.5}$ onde $\theta_{1}>0$ e $\hat{\alpha}=-\frac{a_{2}\left(\theta_{1}\right)}{2 a_{1}}$, com $W_{l}\left(\hat{\alpha}, \theta_{1}\right)=n^{-1}\left\{n_{1} x_{1}^{l} \theta_{1}+\sum_{i=2}^{k} n_{i} x_{i}^{l}\left[\hat{\alpha}\left(1-\frac{x_{j}}{x_{1}}\right)+\frac{x_{j}}{\theta_{1} x_{1}}\right]^{-1}\right\}, l=0,1,2$ e $a_{1}=1078.898501$, isto é, $4 a_{1}=4315.594004$ e $2 a_{1}=2157.797002$,

$\mathrm{a}_{2}\left(\theta_{1}\right)=-2564.967197 \theta_{1}^{-1}+208.642718$

$\mathrm{a}_{3}\left(\theta_{1}\right)=1616.080686 \theta_{1}^{-2}-268.642718 \theta_{1}^{-1}+11.54673$.

A moda desta densidade a posteriori é $\dot{\theta_{1}}=8.75$. No gráfico 7.18 mostramos a densidade a posteriori marginal para $\theta_{1}$. 
7.2 análise do modelo de regressão lincar inuerso - análise clássica e bayesiana - pág. 100 GRÁFICO 7.18

\section{DENSIDADE A POSTERIORI MARCINAL PABA IHETAI}

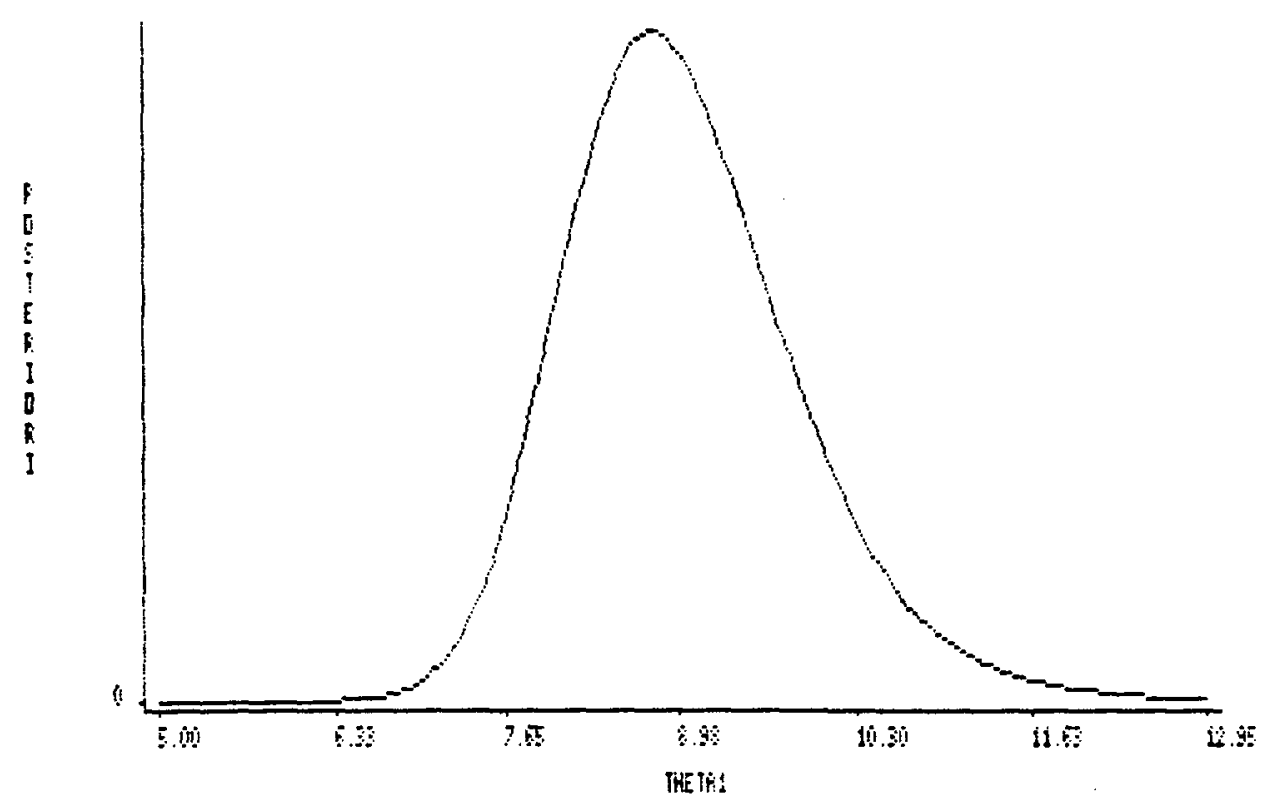

Com $\lambda=94$ conhecido, encontramos também as densidades a posteriores marginais para $\alpha$, $\beta$ e $\theta_{1}$. A densidade a posteriori marginal para $\alpha$ de (4.29) é dada por,

$\pi(\alpha /$ dados $) \propto\left\{\mathrm{W}_{0}(\alpha, \hat{\beta}) \mathrm{W}_{2}(\alpha, \hat{\beta})-\mathrm{W}_{1}^{2}(\alpha, \hat{\beta})\right\}^{1 / 2} \exp \left\{-\left(2873.784777 a^{2}-213.658793 a+18.010499\right)\right\}$ onde $\alpha>0, \lambda$ conhecido e $\dot{\beta}=\frac{\bar{X}-a V_{1}}{V_{2}}, \operatorname{com}_{l}(a, \dot{\beta})=n^{-1} \sum_{i=1}^{3} n_{i} x_{i}^{l}\left(a+\dot{\beta} x_{i}\right)^{-1}, l=0,1,2$ A moda desta densidade a posteriori é $\hat{\alpha}=0.0371$. No gráfico 7.19 mostramos a densidade a posteriori marginal para $\alpha$. 
7.2 análise do modelo de regressâo linear inverso - análise clássica e bayesiana - pág. 101 GRÁFICO 7.19

DENSIDADE A POSTERIORI MARGINAL PARA ALFA CON LANBDA CONHECIDO

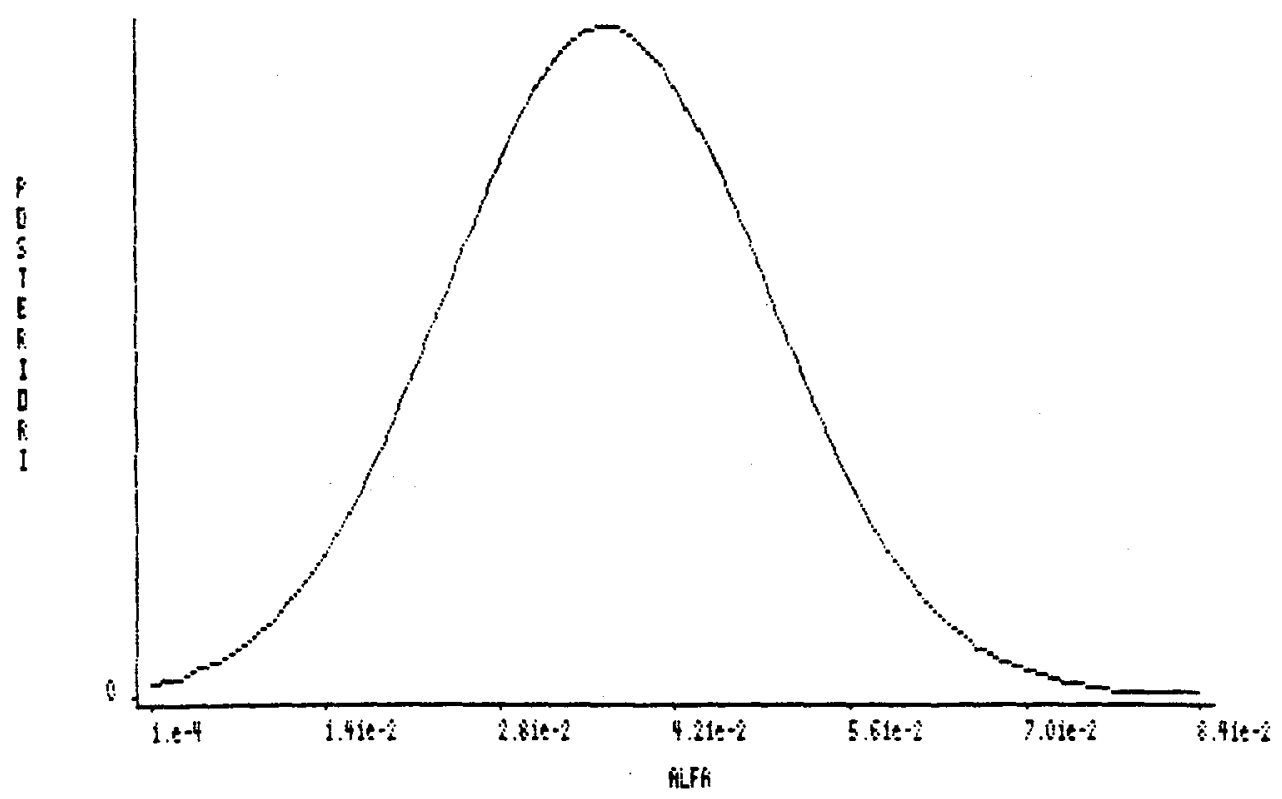

A densidade a posteriori marginal para $\beta$ de (4.30) é dada por,

$\pi(\beta /$ dados $) \propto\left\{\mathrm{W}_{0}(\hat{\alpha}, \beta) \mathrm{W}_{2}(\hat{\alpha}, \beta)-\mathrm{W}_{1}^{2}(\hat{a}, \beta)\right\}^{1 / 2} \exp \left\{-\left(3.7897 \alpha^{2}-55.521113 \alpha+217.341758\right)\right\}$

onde $\beta>0, \lambda$ conhecido e $\hat{\alpha}=\frac{1-\beta \mathrm{V}_{1}}{V_{0}}$, com $W_{l}(\hat{\alpha}, \beta)=\mathrm{n}^{-1} \sum_{i=1}^{3} \mathrm{n}_{i} x_{i}^{l}\left(\hat{\alpha}+\beta \mathrm{x}_{i}\right)^{-1}, \quad l=0,1,2$.

A moda desta densidade a posteriori é $\hat{\beta}=7.35$. No gráfico 7.20 mostramos a densidade a posteriori marginal para $\beta$. 
7.2 análise do modelo de regressảo linear inverso - análise clássica e bayesiana - pág. 102

\section{GRÁFICO 7.20}

DENSIDADE A POSTERIORI MARGINAL PARA BETA COM LAMBDA CONHECIDO

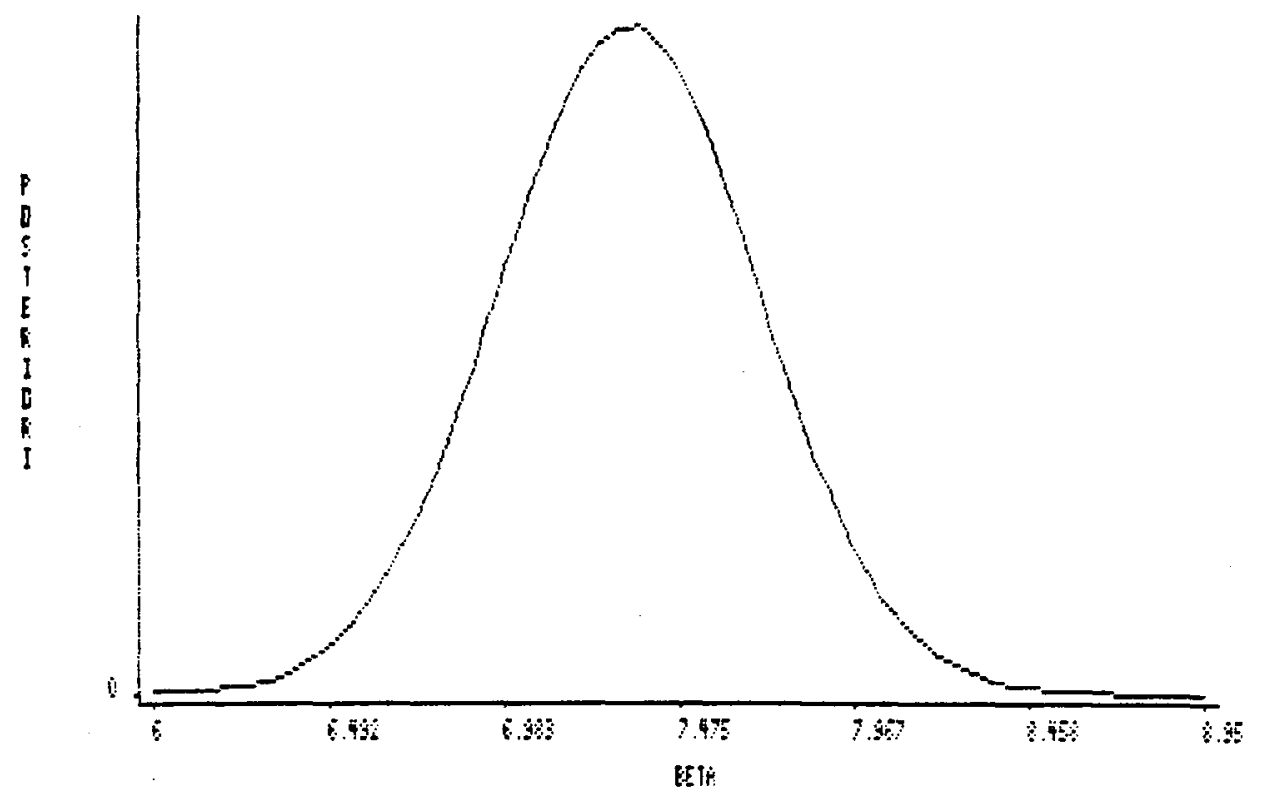

A densidade a posteriori marginal para $\theta_{1}$ de (4.32) é dada por,

$\pi\left(\theta_{1} /\right.$ dados $) \propto \theta_{1}^{-2}\left\{W_{0}\left(\hat{\alpha}, \theta_{1}\right) W_{2}\left(\hat{\alpha}, \theta_{1}\right)-W_{1}^{2}\left(\hat{a}, \theta_{1}\right)\right\}^{1 / 2} \exp \left\{-\left(47 a_{3}\left(\theta_{1}\right)-0.010891 a_{2}^{2}\left(\theta_{1}\right)\right\}\right.$

onde $\theta_{1}>0, \lambda$ conhecido,

e $\hat{\alpha}=-\frac{a_{2}\left(\theta_{1}\right)}{2 a_{1}}$, com $w_{l}\left(\dot{a}, \theta_{1}\right)=n^{-1}\left\{n_{1} x_{1}^{l} \theta_{1}+\sum_{i=2}^{k} n_{i} x_{i}^{l}\left[\hat{\alpha}\left(1-\frac{x_{j}}{x_{1}}\right)+\frac{x_{j}}{\theta_{1} x_{1}}\right]^{-1}\right\}, \imath=0,1,2$

e $a_{1}=1078.898501$, isto é, $4 a_{1}=4315.594004$ e $2 a_{1}=2157.797002$,

$\mathrm{a}_{2}\left(\theta_{1}\right)=-2564.967197 \theta_{1}^{-1}+208.642718$

$\mathrm{a}_{3}\left(\theta_{1}\right)=1616.080686 \theta_{1}^{-2}-268.642718 \theta_{1}^{-1}+11.54673$

e $\frac{\lambda}{8 \mathrm{a}_{1}}=0.010891$

A moda desta densidade a posteriori é $\hat{\theta_{1}}=8.75$. No gráfico 7.21 mostramos a densidade a posteriori marginal para $\theta_{1}$. 
7.2 análise do modelo de regressĩo linear inverso - análise clássica e bayesiana - pág. 109 GRÁFICO 7.21

\section{DDNSIDADE A POSTERIORI MARGINAL PARA IHETA1 COH LAMBDA CONHECIDO}

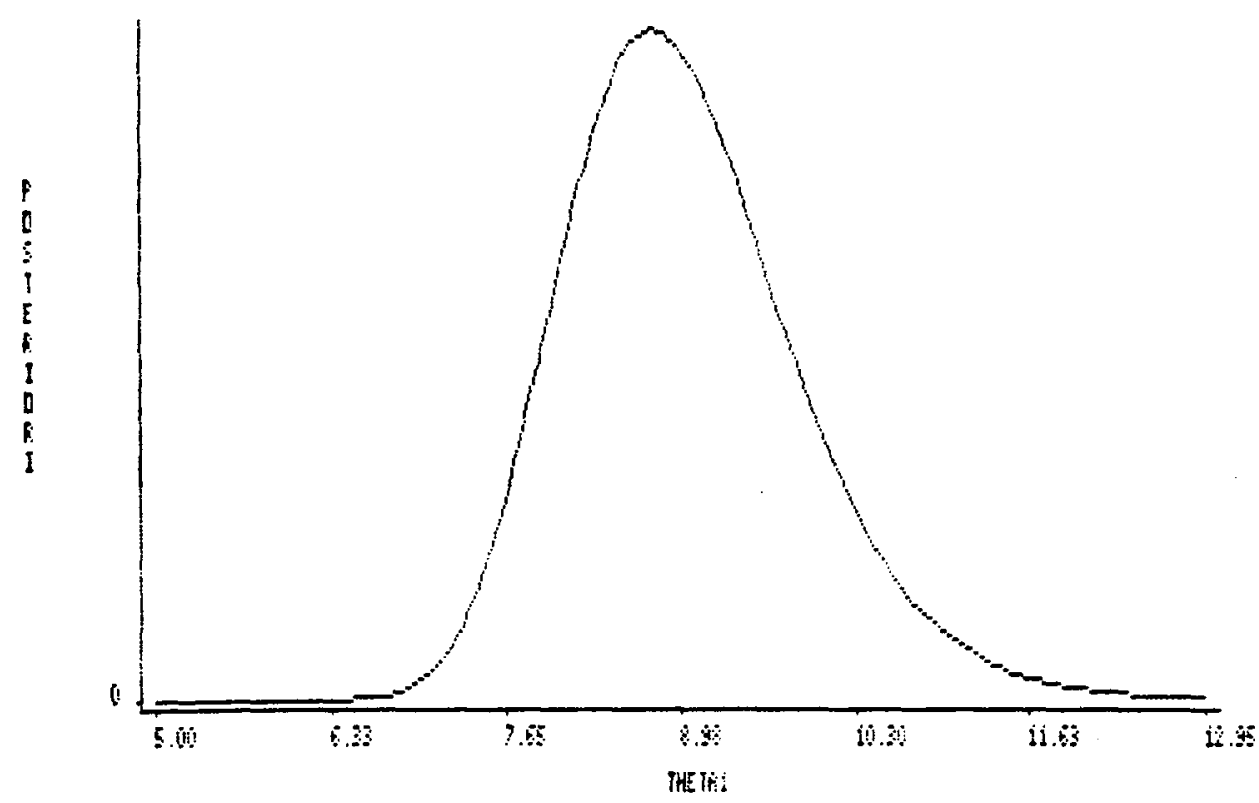

$\mathrm{Na}$ tabela 7.13 , temos intervalos aproximados ao $95 \%$ de confiança e credibilidade para os parâmetros considerando as distribuiçoes limites para as EMV e as densidades a posteriores marginais de interesse.

TABELA 7.13

INTERVALOS DE CONFIANÇA E CREDIBILIDADE PARA OS PARÂMETROS

\begin{tabular}{|l|l|r|l|}
\hline paràmetro & IC $\left(\mathrm{I}_{0}\right)$ & $\mathrm{IC}\left(\mathrm{I}\left(\psi^{\prime}\right)\right)$ & ICR \\
\hline$\alpha$ & $(0.0122 ; 0.0621)$ & $(0.0123 ; 0.0620)$ & $(0.0125 ; 0.0617)$ \\
$\beta$ & $(6.6368 ; 8.0127)$ & $(6.6397 ; 8.0098)$ & $(6.6729 ; 8.0271)$ \\
$\lambda$ & $(49.7260 ; 151.6183)$ & $(49.725 ; 151.618)$ & $(44.760 ; 143.239)$ \\
$\theta_{1}$ & $(7.5290 ; 10.872)$ & $(7.5358 ; 10.857)$ & $(7.2234 ; 10.276)$ \\
\hline
\end{tabular}

Da tabela 7.13 verificamos resultados similares considerando o método clássico assintótico e o método Bayesiano baseados sobre uma densidade a priori não-informativa para os parâmetros. Uma das grandes vantagens do método Bayesiano é relacionado à simplicidade das densidades a posteriori aproximadas, que sâo facilmente implementadas num computador e pode facilitar a análise estatística. Além disso, poderiamos de forma similar, considerar prioris informativas e obter resultados aproximados de forma simples, o que é outra grande vantagem do uso do método Bayesiano. 
7.2 análise do modelo de regressão linear inverso - análise clássica e bayesiana - pág. 104

\subsubsection{USO DA DENSIDADE PREDITIVA EM CONTRŌLE DE QUALIDADE.}

Aplicando os procedimentos obtidos no capitulo 5, mostramos no gráfico 7.22 as densidades preditivas para uma observaşâo futura $\mathrm{Y}_{(31) i}=$ Horas de Falha $/ 1000$ considerando cada nivel de estresse $X_{i}, i=1,2,3$.

\section{GRÁFICO 7.22}

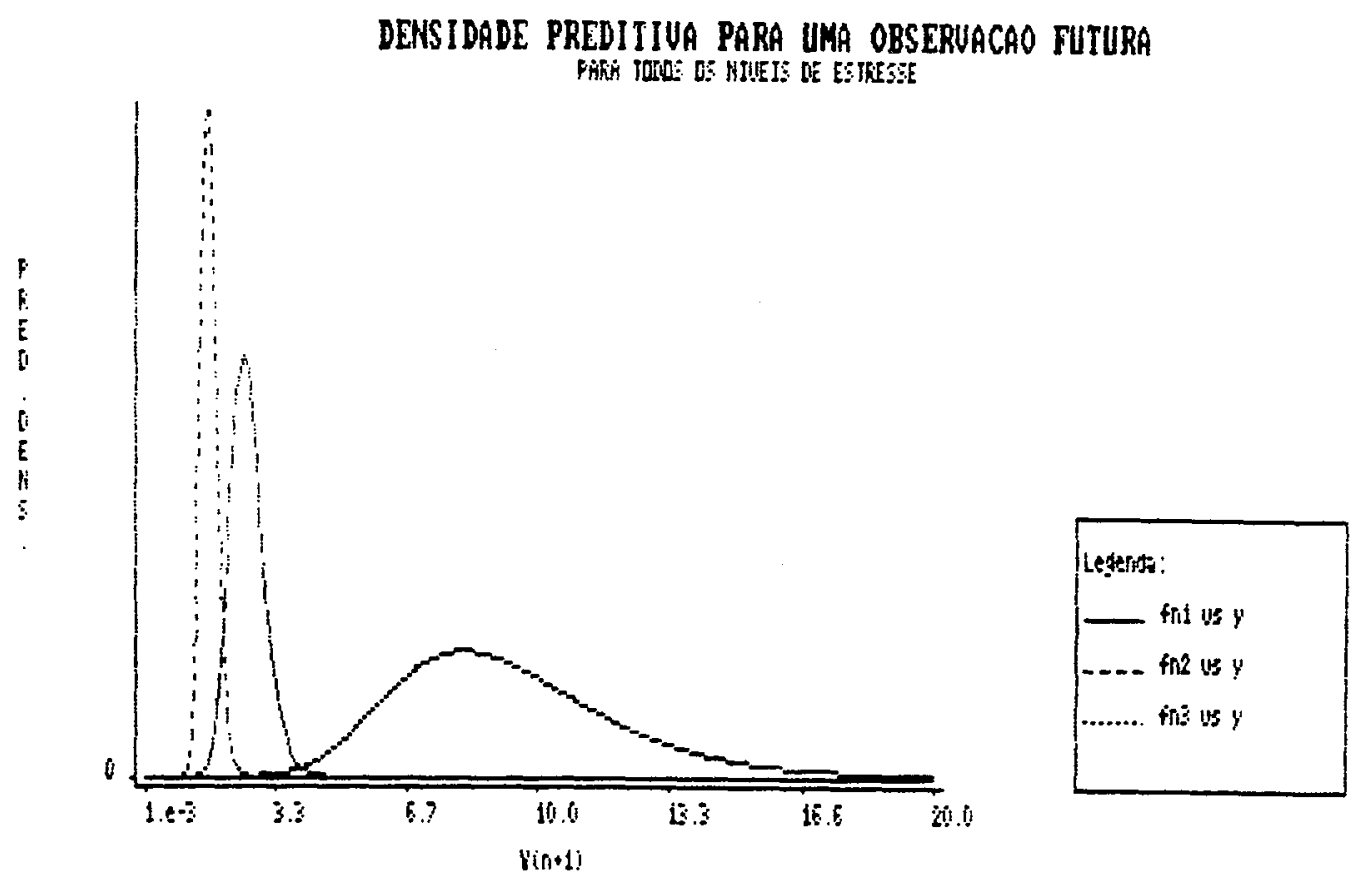

Usamos a densidade preditiva de (5.10) com $\alpha$ e $\beta$ conhecidos para achar $X_{i}$ e $L_{i}$ necessarios para um problema de contrôle de qualidade. Neste caso, fixamos $X_{1}=10^{-8}\left[T_{1}^{3}-180^{3}\right]$, $X_{2}=10^{-8}\left[T_{2}^{3}-180^{3}\right]$ e $X_{3}=10^{-8}\left[T_{3}^{3}-180^{3}\right]$; e achamos $L_{1}, L_{2}$, e $L_{3}$, tal que (5.13) ocorra com uma probabilidade fixa de $1-\gamma=0.95$. Valores obtidos usando o método de Simpson (ver apêndice A2) são dados por:

\begin{tabular}{|c|c|}
\hline $\mathrm{X}_{i}$ & $\mathrm{~L}_{i}$ \\
\hline 0.0103 & 4.99898 \\
0.0482 & 1.96393 \\
0.0799 & 1.32093 \\
\hline
\end{tabular}

Estes resultados podem ser de grande interèsse para engenheiros de qualidade.

Ressaltamos que os resultados destas aplicações numéricas podem ser encontradas em Achcar e Rosales (1991). 
7.3 PLANEJAMENTOS DE EXPERIMENTOS EM TESTES ACELERADOS COM O MODELO DE REGRESSÃO LINEAR INVERSO - UMA ANÁLISE CLÁSSICA E BAYESIANA.

\subsubsection{QUARTO EXEMPLO.}

Neste exemplo apresentamos um conjunto de dados que foi analizado por Shuster e Miura (1972) e introduzidos por Ostle (1963), e que se referem a um experimento fatorial aleatório $2 \times 5$ com 10 replicaçōes por cela. O experimento consiste de um lote de material de isolação, sendo que 10 unidades são cortados longitudinalmente e outros 10 transversalmente. A tabela 7.14 fornece os impactos fortes em libras por pés de teste por unidade.

O uso da distribução Gaussiana inversa é adequado, assim o impacto forte é determinado por construção em niveis acima das falhas ocorridas. A suposição de um nivel critico constante e um parâmetro de difusão constante são válidas neste experimento desde que o mesmo tipo de material de isolação seja testado sobre um especificado critério de falha fixo, visto que a suposição de Shuster e Miura de uma constante $\theta^{2} \sigma$ não tem uma interpretação significativa.

Assumindo o modelo (6.1) para os dados da tabela (7.14), fazemos uma análise clássica e uma análise Bayesiana do modelo. 
TABELA 7.14

\begin{tabular}{|l|ccccc|}
\hline & \multicolumn{5}{|c|}{ Número de Lote } \\
\cline { 2 - 6 } Tipo de Corte & I & II & III & IV & V \\
\hline Longitudinal & 1.15 & 1.16 & 0.79 & 0.96 & 0.49 \\
& 0.84 & 0.85 & 0.68 & 0.82 & 0.61 \\
& 0.88 & 1.00 & 0.64 & 0.98 & 0.59 \\
& 0.91 & 1.08 & 0.72 & 0.93 & 0.51 \\
& 0.86 & 0.80 & 0.63 & 0.81 & 0.53 \\
& 0.88 & 1.01 & 0.59 & 0.79 & 0.72 \\
& 0.92 & 1.14 & 0.81 & 0.79 & 0.67 \\
& 0.87 & 0.87 & 0.65 & 0.86 & 0.47 \\
& 0.93 & 0.97 & 0.64 & 0.84 & 0.44 \\
& 0.95 & 1.09 & 0.75 & 0.92 & 0.48 \\
\hline Transversal & 0.89 & 0.86 & 0.52 & 0.86 & 0.52 \\
& 0.69 & 1.17 & 0.52 & 1.06 & 0.53 \\
& 0.46 & 1.18 & 0.80 & 0.81 & 0.47 \\
& 0.85 & 1.32 & 0.64 & 0.97 & 0.47 \\
& 0.73 & 1.03 & 0.63 & 0.90 & 0.57 \\
& 0.67 & 0.84 & 0.58 & 0.93 & 0.54 \\
& 0.78 & 0.89 & 0.65 & 0.87 & 0.56 \\
& 0.77 & 0.84 & 0.60 & 0.88 & 0.55 \\
& 0.80 & 1.03 & 0.71 & 0.89 & 0.45 \\
& 0.79 & 1.06 & 0.59 & 0.82 & 0.60 \\
\hline
\end{tabular}

\section{ANÁLISE CLÁSSICA.}

Os EMV $\hat{\Phi}=\left(\mu, \alpha_{1}, \beta_{1}, \beta_{2}, \beta_{3}, \beta_{4}\right)^{\prime}$ e $\hat{\sigma}$, são calculadas de (6.18) utilizando os seguintes resultados,

$$
\begin{aligned}
& \mathrm{n}=10 \\
& \mathrm{R}^{*}=136.452461, \\
& \mathrm{D}^{*}=\operatorname{diag}(0.919,0.997,0.690,0.870,0.551,0.743,1.022,0.624,0.899,0.526)^{\prime},
\end{aligned}
$$


$\mathbf{X}=\left[\begin{array}{cccccc}1 & 1 & 1 & 0 & 0 & 0 \\ 1 & 1 & 0 & 1 & 0 & 0 \\ 1 & 1 & 0 & 0 & 1 & 0 \\ 1 & 1 & 0 & 0 & 0 & 1 \\ 1 & 1 & -1 & -1 & -1 & -1 \\ 1 & -1 & 1 & 0 & 0 & 0 \\ 1 & -1 & 0 & 1 & 0 & 0 \\ 1 & -1 & 0 & 0 & 1 & 0 \\ 1 & -1 & 0 & 0 & 0 & 1 \\ 1 & -1 & -1 & -1 & -1 & -1\end{array}\right]$

$\mathbf{X}^{\prime}=\left[\begin{array}{cccccccccc}1 & 1 & 1 & 1 & 1 & 1 & 1 & 1 & 1 & 1 \\ 1 & 1 & 1 & 1 & 1 & -1 & -1 & -1 & -1 & -1 \\ 1 & 0 & 0 & 0 & -1 & 1 & 0 & 0 & 0 & -1 \\ 0 & 1 & 0 & 0 & -1 & 0 & 1 & 0 & 0 & -1 \\ 0 & 0 & 1 & 0 & -1 & 0 & 0 & 1 & 0 & -1 \\ 0 & 0 & 0 & 1 & -1 & 0 & 0 & 0 & 1 & -1\end{array}\right]$

$M=\left[\begin{array}{cccccc}7.841 & 0.213 & 0.585 & 0.942 & 0.237 & 0.692 \\ 0.213 & 7.841 & 0.151 & -0.050 & 0.041 & -0.054 \\ 0.585 & 0.151 & 2.739 & 1.077 & 1.077 & 1.077 \\ 0.942 & -0.050 & 1.077 & 3.096 & 1.077 & 1.077 \\ 0.237 & 0.041 & 1.077 & 1.077 & 2.391 & 1.077 \\ 0.692 & -0.054 & 1.077 & 1.077 & 1.077 & 2.846\end{array}\right]$

$M^{-1}=\left[\begin{array}{cccccc}0.134188 & -0.003852 & -0.013444 & -0.035177 & 0.018212 & -0.021193 \\ -0.003852 & 0.127915 & -0.009694 & 0.005436 & -0.002573 & 0.005949 \\ -0.013444 & -0.009694 & 0.495818 & -0.085735 & -0.138277 & -0.099774 \\ -0.035177 & 0.005436 & -0.085735 & 0.43148 & -0.117303 & -0.07792 \\ 0.018212 & -0.002573 & -0.138277 & -0.117303 & 0.590745 & -0.131312 \\ -0.021193 & 0.005949 & -0.099774 & -0.077792 & -0.131312 & 0.473524\end{array}\right]$ 


$$
\delta=\left[\begin{array}{l}
10 \\
0 \\
0 \\
0 \\
0
\end{array}\right] \text { e } \delta^{\prime}=[10,0,0,0,0]
$$

Assim os EMV são dados por,

$$
\begin{aligned}
& \hat{\Phi}=(1.341884,-0.038518,-0.134436,-0.351772,0.182121,-0.211933)^{\prime} \\
& \hat{\sigma}=0.02264
\end{aligned}
$$

Os EMQ são calculados de (6.30) e (6.31) utilizando os seguintes resultados,

$$
\mathbf{X} \mathbf{X}=\left[\begin{array}{cccccc}
10 & 0 & 0 & 0 & 0 & 0 \\
0 & 10 & 0 & 0 & 0 & 0 \\
0 & 0 & 4 & 2 & 2 & 2 \\
0 & 0 & 2 & 4 & 2 & 2 \\
0 & 0 & 0 & 2 & 4 & 2 \\
0 & 0 & 0 & 2 & 2 & 4
\end{array}\right] \text { e } \quad\left(\mathbf{X}^{\prime} \mathbf{X}\right)^{-1}=\left[\begin{array}{cccccc}
0.1 & 0 & 0 & 0 & 0 & 0 \\
0 & 0.1 & 0 & 0 & 0 & 0 \\
0 & 0 & 0.4 & -0.1 & -0.1 & -0.1 \\
0 & 0 & -0.1 & 0.4 & -0.1 & -0.1 \\
0 & 0 & -0.1 & -0.1 & 0.4 & -0.1 \\
0 & 0 & -0.1 & & -0.1 & 0.4
\end{array}\right]
$$

$$
\mathrm{r}=\left[\begin{array}{l}
1.088139 \\
1.003009 \\
1.449277 \\
1.149425 \\
1.814882 \\
1.345895 \\
0.978474 \\
1.602564 \\
1.112347 \\
1.901141
\end{array}\right]
$$

Assim os EMQ são dados por, 


$$
\begin{aligned}
& \dot{\phi}=(1.344515,-0.043569,-0.127498,-0.353774,0.181405,-0.213629), \\
& \dot{\sigma}=0.02224 .
\end{aligned}
$$

As estimativas correspondentes as médias das celas são calculadas usando a relação $X \Phi=\theta^{-1}$. Essas estimativas e as médias das celas amostrais são dadas na tabela 7.15.

\section{TABELA 7.15}

EMV e EMQ DOS IMPACTOS FORTES MEDIOS

(10 replicaçōes por cela)

\begin{tabular}{|ll|lllll|}
\hline \multirow{2}{*}{ Tipo de Corte } & & \multicolumn{5}{|c|}{ Número de Lote } \\
\cline { 3 - 7 } & & \multicolumn{1}{|c|}{ I } & \multicolumn{1}{|c|}{ II } & III & IV & \multicolumn{1}{c|}{ V } \\
\hline Longitudinal & $\overline{\mathbf{y}}$ & 0.919 & 0.997 & 0.690 & 0.870 & 0.551 \\
& EMV & 0.855483 & 1.050868 & 0.673180 & 0.916227 & 0.549636 \\
& EMQ & 0.852189 & 1.055774 & 0.674604 & 0.919695 & 0.551134 \\
\hline Transversal & $\overline{\mathbf{y}}$ & $\mathbf{0 . 7 4 3}$ & 1.022 & 0.624 & 0.899 & 0.526 \\
& EMV & 0.802590 & 0.972166 & 0.639991 & 0.855821 & 0.527309 \\
& EMQ & 0.793282 & 0.966828 & 0.637150 & 0.851459 & 0.525878 \\
\hline
\end{tabular}

\section{ANÁLISE BAYESIANA.}

A densidade posteriori marginal conjunta para $\Phi=\left(\mu, \alpha_{1}, \beta_{1}, \beta_{2}, \beta_{3}, \beta_{4}\right)^{\prime}$ de (6.36) considerando uma priori não-informativa é dada por,

$$
\begin{aligned}
& \pi(\Phi / \text { dados }) \propto\left\{136.4525-200 \mu+10 \mathrm{~g}\left(\mu, \alpha_{1}, \beta_{1}, \beta_{2}, \beta_{3}, \beta_{4}\right)\right\}^{-50} \\
& \text { onde }-\infty<\mu<\infty,-\infty<\alpha_{1}<\infty,-\infty<\beta_{j}<\infty, j=1,2,3 \mathrm{e} 4, \mathrm{e} \\
& \mathrm{g}\left(\mu, \alpha_{1}, \beta_{1}, \beta_{2}, \beta_{3}, \beta_{4}\right)=7.841 \mu^{2}+0.426 \mu \alpha_{1}+1.17 \mu \beta_{1}+1.884 \mu \beta_{2}+0.474 \mu \beta_{3}+ \\
& +1.384 \mu \beta_{4}+7.841 \alpha_{1}^{2}+0.302 \alpha_{1} \beta_{1}-0.10 \alpha_{1} \beta_{2}+0.082 \alpha_{1} \beta_{3}- \\
& -0.108 \alpha_{1} \beta_{4}+2.739 \beta_{1}^{2}+2.154 \beta_{1} \beta_{2}+2.154 \beta_{1} \beta_{3}+2.154 \beta_{1} \beta_{4}+ \\
& +3.096 \beta_{2}^{2}+2.154 \beta_{2} \beta_{3}+2.154 \beta_{2} \beta_{4}+2.391 \beta_{3}^{2}+2.154 \beta_{3} \beta_{4}+ \\
& +2.846 \beta_{4}^{2} .
\end{aligned}
$$


A moda dessa densidade a posteriori $\left(\tilde{\mu}_{1}, \tilde{a}_{1}, \bar{\beta}_{1}, \bar{\beta}_{2}, \bar{\beta}_{3}, \bar{\beta}_{4}\right)$ é obtida através da resolução do seguinte sistema de equaçōes,

$$
\begin{aligned}
& 15.682 \tilde{\mu}+0.426 \tilde{a}_{1}+1.17 \tilde{\beta}_{1}+1.884 \tilde{\beta}_{2}+1.474 \tilde{\beta}_{3}+1.384 \tilde{\beta}_{4}=20 \\
& 0.426 \tilde{\mu}+15.682 \tilde{\alpha}_{1}+0.312 \tilde{\beta}_{1}-0.100 \tilde{\beta}_{2}+0.082 \tilde{\beta}_{3}-0.0108 \tilde{\beta}_{4}=0 \\
& 1.1700 \tilde{\mu}+0.302 \tilde{\alpha}_{1}+5.478 \tilde{\beta}_{1}+2.154 \tilde{\beta}_{2}+2.154 \tilde{\beta}_{3}+2.154 \tilde{\beta}_{4}=0 \\
& 1.884 \tilde{\mu}-0.100 \tilde{a}_{1}+2.154 \tilde{\beta}_{1}+6.192 \tilde{\beta}_{2}+2.154 \dot{\beta}_{3}+2.154 \tilde{\beta}_{4}=0 \\
& 0.474 \tilde{\mu}+0.082 \tilde{a}_{1}+2.154 \tilde{\beta}_{1}+2.154 \tilde{\beta}_{2}+4.782 \tilde{\beta}_{3}+2.154 \tilde{\beta}_{4}=0 \\
& 1.384 \tilde{\mu}-0.108 \tilde{a}_{1}+2.154 \tilde{\beta}_{1}+2.154 \dot{\beta}_{2}+2.154 \tilde{\beta}_{3}+5.692 \tilde{\beta}_{4}=0
\end{aligned}
$$

Assim, a moda é dada por $\tilde{\Phi}=(1.341884,-0.038518,-0.134436,-0.351772,0.182121,-0.211933)^{\prime}$

A densidade a posteriori marginal para $\sigma$ de (6.37) é dada por,

$$
\pi(\sigma / \text { dados }) \propto \sigma^{-50.0} \exp \left\{-1.13225 \sigma^{-1}\right\}
$$

onde $\sigma>0$. A moda é dada por $\tilde{\sigma}=0.02242$. No gráfico 7.23 mostramos a densidade posteriori marginal para $\sigma$.

\section{GRÁFICO 7.23}

DENSIDADE A POSTERIORI MARGINAL PARA SIGMA

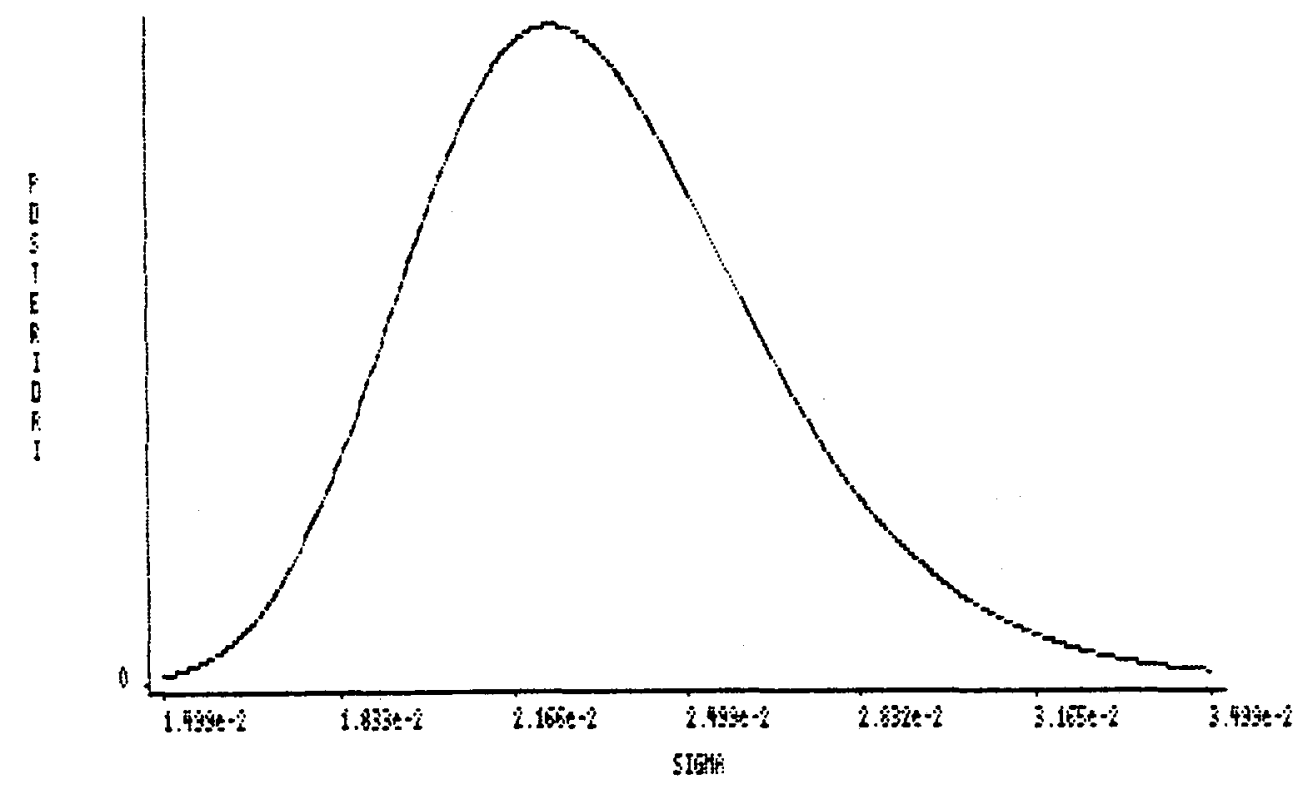


7.9 - planejamentos de experimentos - pág. 111

Na tabela 7.16 apresentamos um resumo das diferentes estimativas, observamos que as estimativas Bayesianas considerando uma priori não-informativa de Jeffreys está muito próxima da estimativa de máxima verossimilhança.

TABELA 7.16

\section{DIFERENTES ESTIMATIVAS PARA OS PARẢMETROS}

\begin{tabular}{|lcccccc|}
\hline & $\alpha_{1}$ & $\beta_{1}$ & $\beta_{2}$ & $\beta_{3}$ & $\beta_{4}$ & $\sigma$ \\
\hline EMV & -0.038518 & -0.134436 & -0.351772 & 0.182121 & -0.211933 & 0.02264 \\
EMQ & $-\mathbf{0 . 0 4 3 5 6 9}$ & -0.127498 & -0.353774 & 0.181405 & -0.213629 & 0.02224 \\
MODA & -0.038518 & -0.134436 & -0.351772 & 0.182121 & -0.211933 & 0.02242 \\
(estim. & & & & & & \\
Bayes.) & & & & & & \\
\hline
\end{tabular}

Podem ser obtidos Intervalos de Confiança e Credibilidade para cada um dos parâmetros utilizando a aproximação normal dos Estimadores de Máxima Verossimilhança. Os resultados são dados na tabela 7.17.

TABELA 7.17

INTERVALOS DE CONFIANÇA E CREDIBILIDADE PARA OS PARÂMETROS

\begin{tabular}{|lccc|}
\hline Parâmetro & IC (95\%) & ICR (95\%) \\
\hline$\mu$ & $(1.307722 ; 1.376046)$ & $(1.308579 ; 1.375189)$ \\
$\alpha_{1}$ & $(-0.071872 ;-0.005164)$ & $(-0.071823 ;-0.005213)$ \\
$\beta_{1}$ & $(-0.200103 ;-0.068769)$ & $(-0.190786 ;-0.078086)$ \\
$\beta_{2}$ & $(-0.413031 ;-0.290513)$ & $(-0.404774 ;-0.29877)$ \\
$\beta_{3}$ & $(0.110443 ; 0.253799)$ & $(0.121809 ; 0.242433)$ \\
$\beta_{4}$ & $(-0.276107 ;-0.147759)$ & $(-0.267214 ;-0.156652)$ \\
$\sigma$ & $(0.016365 ; 0.028915)$ & $(0.016235 ; 0.028605)$ \\
\hline
\end{tabular}

Ressaltamos que os resultados destas aplicações numéricas podem ser encontradas em Achcar e Rosales (1992). 


\section{CAPITULO 8}

\section{CONCLUSÕES E CONSIDERAÇÕES FUTURAS}

O uso do método de Laplace para aproximações de integrais torna viável uma análise Bayesiana do modelo Gaussiano Inverso aplicado a dados de sobrevivència como vimos nos exemplos do capitulo 7. Observamos que as densidades a posteriori de interesse aproximadas pelo método de Laplace são de simples implementação num computador e não exigem grande conhecimento computacional especializado, e podem ser obtidos de forma similar para diferentes densidades a priori, que normalmente incorporam opiniōes dos engenheiros de confiabilidade. É evidente que muitos resultados obtidos nos exemplos coincidem com os resultados clássicos pois as prioris consideradas foram não-informativas.

É importante salientar que num trabalho futuro, poderiam e deveriam ser feitas comparaçōes usando outros métodos de resolução de integrais para avaliar as precisōes dos resultados obtidos neste trabalho. Por exemplo, poderiamos usar o método de Simpson para calcular as densidades a posteriori de interesse ou o método de Monte Carlo. Além disso, seria importante um estudo detalhado sobre o erro das aproximaçôes obtidas.

Também, poderiamos sob o enfoque de testes acelerados, usar o método de Laplace para aproximar momentos a posteriori de interesse. Assim, poderiamos estimar funções dos parâmetros sob o nivel usual de estresse, que é o objetivo principal de um experimento de testes acelerados.

Em síntese, as aproximaçōes obtidas podem ser de grande interesse prático para a área de confiabilidade incorporando opiniôes de especialistas e mesmo com a presença de dados censurados, pois os resultados aproximados poderiam ser implementados nessas situações. 


\section{REFERÊNCIAS BIBLIOGRÁFICAS}

- Achcar, J. A. (1990). Use of Preditictive Densities in Quality Control With Accelerated Life Tests Assuming a Power Rule Model and an Exponential Distributions, Relatório Téenico, $n$ 우 76/90, ICMS - USP, São Carlos.

- Achcar, J. A., Bolfarine, H. Rodrigues, J. (1987). Inverse Gaussian Distribution: a Bayesian Approach. Thechnical Report no 8709, IME-USP, São Paulo, S. P.

- Achcar, J. A., Bolfarine, H. and Rodrigues, J. (1991). Análise de Sobrevivência. 2a Escola de Modelos de Regressâo, IM - UFRJ.

- Achcar, J. A. and Rosales, O.L.A. (1991). A Bayesian Approach for Accelerated Life Tests Assuming an Inverse Gaussian Distribution. Relatório Técnico, no 94/91, ICMSC - USP, São Carlos.

- Achcar, J. A. and Rosales, O.L.A. (1992). Use of Bayesian Methods in the Analysis of Two-Factor experiments under Inverse Gaussian Distribution. Relatório Técnico, no 119/92, ICMSC - USP, São Carlos.

- Al Hussaini, E. K. and ABD-El-Hakin, N. S. (1981). Bivariate Inverse Gaussian Distributions. Annals Mathematical Statistics, vol. 33, part A, 57-66.

- Banerjee, A. K. and Bhattacharyya, G. K. (1979). Bayesian Results for the Inverse Gaussian Distribution with an Applications. Technometrics, vol. 21, $\mathrm{n}^{\circ}=2,247-251$.

- Bhattacharyya, G. K., Fries, A. (1981). Inverse Gaussian Regression and Accelerated Life Tests. In Proccedings of the Special Topics Meeting on Survival Analysis, 138th Meeting of the Institute of Mathematical Statistics, Columbus, Ohio, oct. 1981, $101-117$.

- Bhattacharyya, G. K. and Fries, A. (1982). Fatigue Failure Models - Birnbaum-Saunders vs. Inverse Gaussian. IEEE Transactions on Reliability, vol. R-31, no 5. 
- Box, G. E. P. and Tiao, G. C. (1973). Bayesian Inference in Statistical Analysis. Addison - Wesley, New York.

- Cheng, R.C.H. and Amin, N.A.K. (1981). Maximum Likelihood Estimation of Parameters in the Inverse Gaussian Distribution, With Unknown Origin. Technometrics, vol. 23, 므 3.

- Cheng, R.C.H. (1984). Generation of Inverse Gaussian Variates with Given Sample Mean and Dispersion. Appl. Statist., 33, nํㅜ 3, 309-316.

- Chhikara, R. S. and Folks, J. L. (1974). Estimation of the Inverse Gaussian Distribution Function. JASA, vol. 69, $n=345,250-254$.

- Chhikara, R. S. and Folks, J. L. (1975). Statistical Distributions Related to the Inverse Gaussian. Communications in Statistics, 4(12), 1081-1091.

- Chhikara, R. S. and Folks, J. L. (1976). Optimum Test Procedures for the Mean of First Passage Time Distribution in Brownian Motion with Positive Drift (Inverse Gaussian Distribution). Technometrics, vol. 18, $\mathrm{n}^{\circ} 2,189-193$.

- Chhikara, R. S. and Folks, J. L. (1977). The Inverse Gaussian Distribution as a Lifetime Model. Technometrics, vol. 19, no 4, $461-468$.

- Chhikara, R. S. and Folks, J. L. (1989). The Inverse Gaussian Distribution - Theory, Methodology and Applications. Marcel Dekker, Inc. New York and Basel.

- Cox, D. R. and Miller, H. D. (1965). The Theory of Stochastic Processes. Chapman and Hall.

- Dias, T. C. M. (1992). Testes Acelerados Assumindo um Modelo de Lei de Pótencia Inversa e Dados com Censuras do Tipo II. Tese de Mestrado, ICMSC - USP, São Carlos.

- Doksum, K. A. and Hóyland, A. (1992). Models for Variable-Stress Accelerated Life Testing Experiments Based on Wiener Processes and the Inverse Gaussian Distribution. Technometrics, vol. $34, \mathrm{n}^{\mathbf{0}} 1$. 
referências bibliográficas - pág. 115

- Folks, J. L. and Chhikara, R. S. (1978). The inverse Gaussian Distribution and its Statistical Application - A Review. J. R. Statistical Soc. B, 40, no 3, 263-289.

- Fries, A. and Bhattacharyya, G. K. (1983). Analysis of Two Factor Experiments Under an Inverse Gaussian Model. JASA, vol. 78, $\mathrm{n} \cong$ 384, $820-826$.

- Johnson, N. L. and Kotz, S. (1970). Distributions in Statistics: Continuos Univariate Distributions I. Honhgton - Mifflin, Boston.

- Khatri, C. G. (1961). A Characterization of the Inverse Gaussian Distribution. Annals Mathematical Statistics, 33, $800-803$.

- Kass, R. E., Tierney, L. and Kadane, J. B. (1990). The Validity of Posterior Expansions Based on Laplace's Method. in Essays in Honor of George A. Barnard, ed. Hodges, Amsterdam: North - Holland.

- Lawless, J. F. (1982). Statistical Models and Methods for Lifetime Data. John Wiley \& Sons, New York.

- Louzada Neto, F. (1991). Testes de Sobrevivência Acelerados: Uma Análise Bayesiana do Modelo de Eyring. Tese de Mestrado, ICMSC - USP, São Carlos.

- Mann, N. R., Schafer, R. E. and Singpurwalla, N. A. (1974). Methods for Statistical Analysis of Reliability and Life Data. John Wiley \& Sons, New York.

- Martz, H.F. and Waller, R. A. (1982). Bayesian Reability Analysis. John Wiley \& Sons, New York.

- Miller Jr., R. G. (1981). Survival Analysis. John Wiley \& Sons, New York.

- Mood, A. M., Graybill, F. A. and Boes, D. C. (1974). Introduction to the Theory of Statistics, $3^{a}$ ed., McGraw-Hill, New York.

- Nelson, W. (1971). Analysis of Accelerated Life Test Data - Part I: The Arrhenius Model and Graphical Methods. IEEE, Transations on Electrical Insulation, Vol. EI-6, 4, dez./1971. 
- Nelson, W. (1990). Accelerated Testing - Statistical Models, Test Plans, and Data Analyses. John Wiley, New York.

- Padgett, W.J. (1979). Confidence Bounds on Reliability dor the Inverse Gaussian Model. IEEE Transactions on Reliability, vol. R-28, $\mathrm{n}^{0} 2$.

- Padgett, W.J. (1981). Bayes Estimation of Reliability for the Inverse Gaussian Model. IEEE Transaction on Reliability, vol. R-30, $n^{0} 4$.

- Press, S. J. (1989). Bayesian Statistics: Principles, Models and Applications. John Wiley \& Sons, New York.

- Shuster, J. (1968). On the Inverse Gaussian Distribution Function. JASA, 63, 1514-1516.

- Shuster, J. J. and Miura, C. (1972). Two-Way Analysis of Reciprocals. Biometrika, vol. 59, $478-481$

- Tierney, L. and Kadane, J. B. (1986). Accurate Approximations for Posterior Moments and Marginal Densities. JASA, 81, $82-86$.

- Tierney, L. and Kadane, J. B. (1989). Fully Exponential Laplace Aproximations to Expectations and Variances of Nonpositive Functions. JASA, 84, 710-716.

- Tweedie, M. C. K. (1957a). Statistical Properties of Inverse Gaussian Distributions I. Annals Mathematical Statistics, 28, 362 - 377.

- Tweedie, M. C. K. (1957b). Statistical Properties of Inverse Gaussian Distributions II. Annals Mathematical Statistics, 28, $696-705$.

- Zellen, M. (1959). Factorial Experiments in Life Testing. Technometrics, vol. 1, no 3, 269-288.

- Zellner, A. (1971). An Introduction to Bayesian Inference in Econometrics. John Wiley \& Sons, New York.

- Wasan, M. T. and Roy, L. K. (1969). Tables of Inverse Gaussian Percentage Points. Technometrics, vol. $11, n^{\circ} 3,591-604$. 


\section{APÊNDICE A1}

\section{O MÉTODO DE LAPLACE}

\section{A1 O MÉTODO DE LAPLACE.}

Assumindo h uma função bem comportada de um parámetro m-dimensional com $-\mathrm{h}$ tendo um máximo em $\hat{\theta}$, o método de Laplace aproxima uma integral da forma,

$$
I=\int f(\theta) \exp [-\operatorname{nh}(\theta)] d \theta
$$

expandindo h e f em uma serie de Taylor em törno de $\dot{\theta}$ ( ver por exemplo, Kass, Tierney e Kadane,1990).

Considerando primeiro o caso uni-dimensional, o método de Laplace fornece a seguinte aproximação,

$$
\hat{\mathbf{I}} \simeq\left(\frac{2 \pi}{n}\right)^{1 / 2} \sigma \mathrm{f}(\hat{\theta}) \exp [-\operatorname{nh}(\hat{\theta})]
$$

onde $\sigma=\left\{h^{\prime \prime}(\hat{\theta})\right\}^{-1 / 2}$.

No caso multiparamétrico, $\operatorname{com} \theta \in \mathbf{R}^{m}$, temos,

$$
\hat{\mathrm{I}} \simeq(2 \pi)^{m / 2}\left\{\operatorname{det}\left(n D^{2} \mathrm{~h}(\hat{\theta})\right)\right\}^{-1 / 2} \mathrm{f}(\hat{\theta}) \exp [-\operatorname{nh}(\hat{\theta})]
$$

onde $\hat{\theta}$ maximiza $-h(\theta)$ e $D^{2} h(\theta)$ é a matriz hessiana de h evaluada em $\dot{\theta}$.

Os erros dessas aproximaçōes são pesquisados por Kass, Tierney e Kadane (1990). Um caso especial das aproximações de Laplace é dado por integrais da forma $\int \exp \{-\operatorname{nh}(\theta)\} \mathrm{d} \theta$ (ver por exemplo, Tierney e Kadane, 1986; Tierney, Kass e Kadane, 1989). 


\section{APÊNDICE A2}

\section{O MÉTODO DE SIMPSON}

\section{A2.1 O MÉTODO DE SIMPSON.}

Uma boa aproximação para o cálculo da integral de uma função é dada pela fórmula de Simpson. É conhecido como o Método de Newton-Cotes e é um método de quadratura. Ele surge da idéia de calcular a integral de $f(x)$ em um certo número finito de pontos, faz uma interpolação polinomial e integra o polinômio, ao invés de $f(x)$.

$O$ valor da integral de $f(x)$ no intervalo $\left(x_{0} ; x_{n}\right)$ é dada por:

$$
g_{s}=\frac{h}{3}\left(f_{0}+4 f_{1}+2 f_{2}+4 f_{3}+\ldots+2 f_{n-2}+4 f_{n-1}+f_{n}\right)
$$

onde $h$ é o incremento entre os pontos $x_{0}$ e $x_{n}$. É dada por $h=\frac{x_{n}-x_{0}}{n}, n$ é o número de intervalos,

$$
\begin{aligned}
& f_{0}=f\left(x_{0}\right) \\
& f_{1}=f\left(x_{0}+h\right)=f\left(x_{1}\right) \\
& f_{2}=f\left(x_{1}+h\right)=f\left(x_{2}\right) \\
& \cdot \\
& f_{n}=f\left(x_{n-1}+h\right)=f\left(x_{n}\right)
\end{aligned}
$$

A função (A2.1) é conhecida como a fórmula de 1/3 de Simpson. 
Apénd. A2 pág. 119

\section{A2.2 CÁlCULO DO ERRO.}

Como em qualquer método numérico que apresenta uma solução aproximada, o erro para o método de Simpson pode ser determinado. Considere $\epsilon$ como o erro cometido em cada $f(x)$. Assim o erro total no cálculo da integral é dado por,

$$
E_{t}=\left(\frac{h^{4}}{180} f^{i v}(\xi)+\epsilon\right)\left(x_{n}-x_{0}\right)
$$

onde : $\quad f^{i v}(\xi)$ é a quarta derivada de $f(x)$,

$\xi$ é um ponto entre $x+h$ e $x-h$,

$x_{n}$ é o limite superior da integral $e$

$x_{0}$ é o limite inferior da integral. 


\section{APÊNDICE A3}

\section{INEERÉNCIAS PARA OS PARÁMETROS A PARTIR DA TEORIA ASSINTÓTICA}

\section{A3.1 ANÁLISE CLÁSSICA.}

As inferências para o vetor de parâmetros $\psi=(\alpha, \beta, \lambda)^{\prime}$ serão baseados nas propriedades assintóticas (grandes amostras) dos estimadores de máxima verossimilhança (EMV) cujo vetor de estimativas é dado por $\hat{\psi}=(\hat{\alpha}, \hat{\beta}, \hat{\lambda})^{\prime}$ (Mood, Graybill e Boes (1974), Lawless (1982)). A teoría assintótica dos EMV sobre a qual a aproximação normal é baseada requer de certas condições de regularidade baseadas no ajustamento da função de verossimilhança.

Assim, para obter intervalos de confiança ou testes de hipôteses para $\psi$ usamos a aproximação assintótica normal dada por,

$$
\frac{\dot{\psi}-\psi}{\mathrm{I}(\bar{\psi})^{-1 / 2}} \sim \mathrm{N}(0,1)
$$

onde I $(\hat{\psi})$ é a matriz de informação de Fisher, definida como,

$$
I(\psi)=E\left\{-\frac{\partial^{2} I(\psi)}{\partial \psi^{2}}\right\} .
$$

Como uma alternativa para (A3.1) podemos utilizar a aproximação assintótica normal equivalente dada por,

$$
\frac{\dot{\psi}-\psi}{\mathrm{I}_{0}^{-1 / 2}} \sim \mathrm{N}(0,1)
$$

onde $I_{0}$ é a matriz de informação observada, definida como,

$$
I_{0}=\left.\left\{-\frac{\partial^{2} J(\psi)}{\partial \psi^{2}}\right\}\right|_{\psi=\dot{\psi}}
$$


Muitas vezes essas aproximaçōes não são muitas boas para amostras pequenas ou moderadas. Quando não temos amostras grandes existem procedimentos alternativos que podem ser utilizados para pequenas amostras (Lawless, 1982).

Da normalidade assintótica dos EMV encontramos intervalos de confiança (IC) aproximados para os parâmetros.

Um intervalo de confiança aproximado para $\psi$ é dado a partir de,

$$
\hat{\psi}=(\hat{\alpha}, \hat{\beta}, \hat{\lambda})^{\prime} \underline{a}\left\{(\alpha, \beta, \lambda) ; I_{0}^{-1}(\psi)\right\}
$$

Por exemplo, um IC $100(1-\gamma) \%$ aproximado para $\alpha$ pode ser construido a partir de,

$$
\hat{\alpha} \stackrel{a}{a} \mathbf{N}\{\alpha, \hat{\mathrm{V}}(\hat{\alpha})\}
$$

isto é,

$$
\mathrm{P}\left(-\mathrm{Z}_{\gamma / 2}<\mathrm{Z}<\mathrm{Z}_{\gamma / 2}\right)=1-\gamma
$$

onde,

$$
\mathrm{Z}=\frac{\hat{\alpha}-\alpha}{\sqrt{\hat{\mathrm{V}}(\hat{\alpha})}} \sim \mathrm{N}(\mathbf{0}, 1)
$$

Assim,

$$
-\mathrm{Z}_{\gamma / 2}<\frac{\hat{\alpha}-\alpha}{\sqrt{\hat{\mathrm{V}}(\hat{\alpha})}}<\mathrm{Z}_{\gamma / 2}
$$

ou,

$$
-\mathrm{Z}_{\gamma / 2} \sqrt{\hat{\mathrm{V}}(\hat{\alpha})}<\hat{\alpha}-\alpha<\mathrm{Z}_{\gamma / 2} \sqrt{\hat{\mathrm{V}}(\hat{\alpha})}
$$

e,

$$
\hat{\alpha}-Z_{\gamma / 2} \sqrt{\hat{V}(\hat{\alpha})}<\alpha<\hat{\alpha}+Z_{\gamma / 2} \sqrt{\hat{V}(\alpha)}
$$

onde $Z_{\gamma / 2}$ é o percentil de uma distribuição normal padronizada. 
Portanto, os limites para um intervalo de confiança $100(1-\gamma) \%$ aproximado para $\alpha$, são dados por,

$$
\operatorname{IC}(\alpha)=\left[\hat{\alpha}-Z_{\gamma / 2} \sqrt{\hat{V}(\hat{\alpha})}, \dot{\alpha}+Z_{\gamma / 2} \sqrt{\hat{V}(\hat{\alpha})}\right]
$$

De maneira análoga obtemos intervalos de confiança para os outros parámetros de interesse. $O$ procedimento é similar usando a matriz de informação de Fisher.

\section{A3.2 ANÁLISE BAYESIANA.}

Usando algumas propriedades da teoria assintótica para as densidades a posteriori marginais dos parâmetros, podemos considerar,

$$
\pi(\alpha / \text { dados }) \alpha \pi(\alpha) L(\alpha)
$$

isto é,

$$
\pi(\alpha / \text { dados }) \propto \pi(\alpha) \exp \{\log L(\alpha)\}
$$

onde $\pi(\alpha)$ é a densidade a priori não-informativa e $L(\alpha)$ é a função de verossimilhança.

Assim, assumindo que ambos $\pi(\alpha)$ e $\mathrm{L}(\alpha)$ sejam diferentes de zero no espaço paramétrico, as derivadas sejam continuas e que $\pi$ ( $\alpha /$ dados) tenha um ponto de máximo em $\dot{\alpha}$, então expandido $\log \pi$ ( $\alpha /$ dados) em (A3.4) por uma série de Taylor em torno de $\hat{\alpha}$, temos,

$$
\begin{aligned}
& \log \pi(\alpha / \text { dados }) \alpha
\end{aligned}
$$

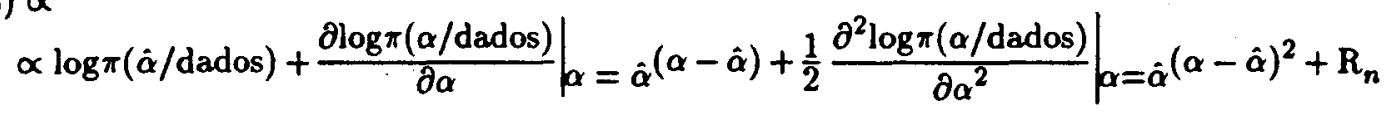

Como $\alpha=\hat{\alpha}$ é a moda da densidade posteriori, então o 20 termo é igual a zero. Portanto,

$$
\begin{aligned}
\log \pi(\alpha / \text { dados }) & \propto \log \pi(\hat{\alpha} / \text { dados })+\left.\frac{1}{2} \frac{\partial^{2} \log \pi(\alpha / \text { dados })}{\partial \alpha^{2}}\right|_{\alpha=\hat{\alpha}}(\alpha-\hat{\alpha})^{2} \\
& \propto \log \pi(\hat{\alpha} / \text { dados })-\frac{1}{2}\left(-\left.\frac{\partial^{2} \log \pi(\alpha / \text { dados })}{\partial \alpha^{2}}\right|_{\alpha=\hat{\alpha}}\right)(\alpha-\hat{\alpha})^{2}
\end{aligned}
$$

Isto é, 
$\pi(\alpha /$ dados $) \alpha \pi(\hat{\alpha} /$ dados $) \exp \left\{-\frac{1}{2}\left(-\left.\frac{\partial^{2} \log \pi(\alpha / \text { dados })}{\partial \alpha^{2}}\right|_{\alpha=\hat{\alpha}}\right)(\alpha-\hat{\alpha})^{2}\right\}$.

dai,

$$
\pi(\alpha / \text { dados }) \propto \exp \left\{-\frac{1}{2}\left(-\frac{\partial^{2} \log \pi(\alpha / \text { dados })}{\partial \alpha^{2}} \mid \alpha=\dot{\alpha}\right)^{-1}(\alpha-\dot{\alpha})^{2}\right\}
$$

Portanto,

$$
\pi(\alpha / \text { dados }) \stackrel{a}{\sim} \mathrm{N}\left[\hat{\alpha},\left(-\left.\frac{\partial^{2} \log \pi(\alpha / \text { dados })}{\partial \alpha^{2}}\right|_{\alpha=\dot{\alpha}}\right)^{-1}\right]
$$

Logo um ICR 100(1 - $) \%$ aproximado para $\alpha$ é dado por,

$$
\mathrm{P}\left(-\mathrm{Z}_{\gamma / 2}<\mathrm{Z}<\mathrm{Z}_{\gamma / 2}\right)=1-\gamma,
$$

onde,

$$
\mathrm{Z}=\frac{\alpha-\hat{\alpha}}{\left(-\left.\frac{\partial^{2} \log \pi(\alpha / \text { dados })}{\partial \alpha^{2}}\right|_{\alpha=\hat{\alpha}}\right)^{-1 / 2}} \sim \mathrm{N}(0,1)
$$

Assim,

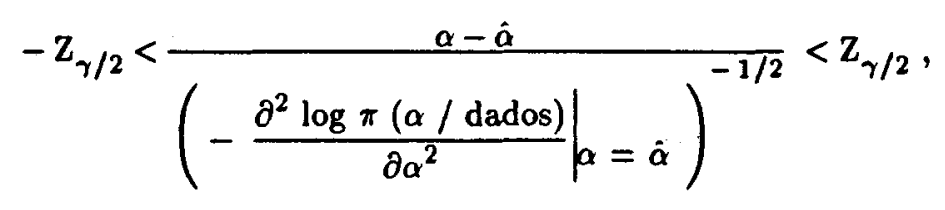

ou,

$-\mathrm{Z}_{\gamma / 2}\left(-\left.\frac{\partial^{2} \log \pi(\alpha / \text { dados })}{\partial \alpha^{2}}\right|_{\alpha=\hat{\alpha}}\right)^{-1 / 2}<\alpha-\hat{\alpha}<\mathrm{Z}_{\gamma / 2}\left(-\left.\frac{\partial^{2} \log \pi(\alpha / \text { dados })}{\partial \alpha^{2}}\right|_{\alpha=\hat{\alpha}}\right)^{-1 / 2}$

e, 
Apénd. A9 pág. 121

$\hat{\alpha}-Z_{\gamma / 2}\left(-\left.\frac{\partial^{2} \log \pi(\alpha / \text { dados })}{\partial \alpha^{2}}\right|_{\alpha=\dot{\alpha}}\right)^{-1 / 2}<\alpha<\dot{\alpha}+Z_{\gamma / 2}\left(-\left.\frac{\partial^{2} \log \pi(\alpha / \text { dados })}{\partial \alpha^{2}}\right|_{\alpha=\dot{\alpha}}\right)^{-1 / 2}$

onde $Z_{\gamma / 2}$ é o percentil de uma distribuição normal padronizada.

Portanto, os limites para um intervalo de credibilidade $100(1-\gamma) \%$ aproximado para $\alpha$, sâo dados por,

$\operatorname{ICR}(\alpha)=\left[\hat{\alpha}-Z_{\gamma / 2}\left(-\left.\frac{\partial^{2} \log \pi(\alpha / \text { dados })}{\partial \alpha^{2}}\right|_{\alpha=\hat{\alpha}}\right)^{-1 / 2}, \hat{\alpha}+Z_{\gamma / 2}\left(-\left.\frac{\partial^{2} \log \pi(\alpha / \text { dados })}{\partial \alpha^{2}}\right|_{\alpha=\hat{\alpha}}\right)^{-1 / 2}\right]$

De maneira análoga obtemos intervalos de credibilidade para os outros parâmetros de interesse. Logo, o procedimento é similar usando a matriz de informação de Fisher. 


\section{APÊNDICE A4}

\section{PROGRAMAS COMPUTACIONAIS}

Neste apêndice apresentamos alguns programas computacionais utilizados nesta dissertação. Eles foram desenvolvidos utilizando o pacote estatistico SOC - Software Científico (desenvolvido e distribuído pela NTIA/EMBRAPA). Foram utilizados alguns módulos principais do pacote, como por exemplo o GENESE, CM, IMPRIME e GRÁfICO. O programa apresentado em A.4.1 foi utilizado para encontrar a densidade a posteriori marginal para $\mu$ considerando uma priori de referência. $O$ segundo programa, A.4.2 foi utilizado para encontrar um contorno da densidade a posteriori conjunta para $\mu$ e $\lambda$. O programa A.4.3 foi utilizado para encontrar a densidade preditiva para uma observação futura para todos os niveis de estresse.

\section{A.4.1 - DENSIDADE A POSTERIORI MARGINAL PARA $\mu$.}

\section{PROGRAMA PARA GERAR OS PONTOS DA DENSIDADE.}

genese \$1funcmur 1

num mu $11 \mathrm{n}$ yb lambe $\mathbf{g} b$ xi $s \mathbf{k}$;

$\{n=30$;

$y b=1.037$;

lambe $=2.902786$;

$\mathrm{g}=8.717829 \mathrm{e}+010$;

$b=0.469498$;

$\mathrm{xi}=\operatorname{sqrt}(((\mathrm{n}-1) * \operatorname{lambe}) / \mathrm{yb})$;

$s=1-\operatorname{tprob}(x i,(n-1))$;

$k=\left(\operatorname{sqrt}(y b * l a m b e) *(n /(2 * l a m b e))^{-}(n / 2)\right) /(s * b * g) ;$

$\mathrm{mu}=0.001$;

enquanto ( $m u<=2$ ) \{

$11=k * g *(n /(2 * l a m b e))^{-}(-(n / 2)) *\left(1+\left(y b * \operatorname{lambe} *\left(m u-y b^{*}(-1)\right)^{2}\right)\right)^{*}(-(n / 2))$;

grave;

$\mathrm{mu}=\mathrm{mu}+0.01 ;\}$ \} 
PROGRAMA PARA CONSTRUIR GRÁfICOS DA DENSIDADE.

grafico \$1funcmur1

graf mu*11=(11);

tII DENSIDADE A POSTERIORI MARGINAL PARA MU;

t12 (exemplo 1);

rot1 $\mathrm{MU}$;

rot2 POSTERIORI;

eixo ver $=0$ ate 00 ;

了

A.4.2 - CONTORNO DA DENSIDADE A POSTERIORI CONJUNTA PARA $\mu$ e $\lambda$.

PROGRAMA PARA GERAR OS PONTOS DO CONTORNO DA DENSIDADE.

genese \$1cont1

num la a b c1 c2 c3 mu11 mu12 mu21 mu22 mu31 mu32;

$\{\mathrm{la}=0.001$;

enquanto (la $<=6)\{$

$a=-15.56 * 1 \mathrm{a}$;

$\mathrm{b}=30.04 * 1 \mathrm{a}$;

$c 1=(14 * \log (\operatorname{la}))-(19.65 * \operatorname{la})+0.745$;

$c 2=(14 * \log (\mathrm{la}))-(19.65 * \operatorname{la})+1.345$;

$\mathrm{c} 3=(14 * \log (\mathrm{la}))-(19.65 * \mathrm{la})+3.045$;

mu11 $=(-b+\operatorname{sqrt}(b-2-4 * a * c 1)) /(2 * a)$;

mu12=(-b-sqrt $(b 2-4 * a * c 1)) /(2 * a)$;

$\mathrm{mu} 21=(-\mathrm{b}+\mathrm{sqrt}(\mathrm{b} 2-4 * \mathrm{a} * \mathrm{c} 2)) /(2 * \mathrm{a})$;

mu22=(-b-sqrt $(b 2-4 * a * c 2)) /(2 * a)$;

mu31 $=\left(-b+\operatorname{sgrt}\left(b^{2} 2-4 * a * c 3\right)\right) /(2 * a)$;

mu32=(-b-sqrt $(b 2-4 * a * c 3)) /(2 * a)$;

grave;

$l a=l a+0.05 ;\}\}$ 
PROGRAMA PARA CONSTRUIR GRÁFICOS DE CONTORNOS DA DENSIDADE grafico $\$ 1$ cont 1

graf mul1*la=(11) mu12*la=(11) mu21*la=(11) mu22*la=(11)

mu31*la=(11) mu32*la=(11);

tI1 CONTORNOS DA POSTERIORI CONJUNTA MU E LAMBDA;

t12 (exemplo 1);

rot1 $\mathrm{MU}$;

rot2 LAMBDA;

)

A.4.3 - DENSIDADE PREDITIVA PARA UMA OBSERVAÇÃO FUTURA

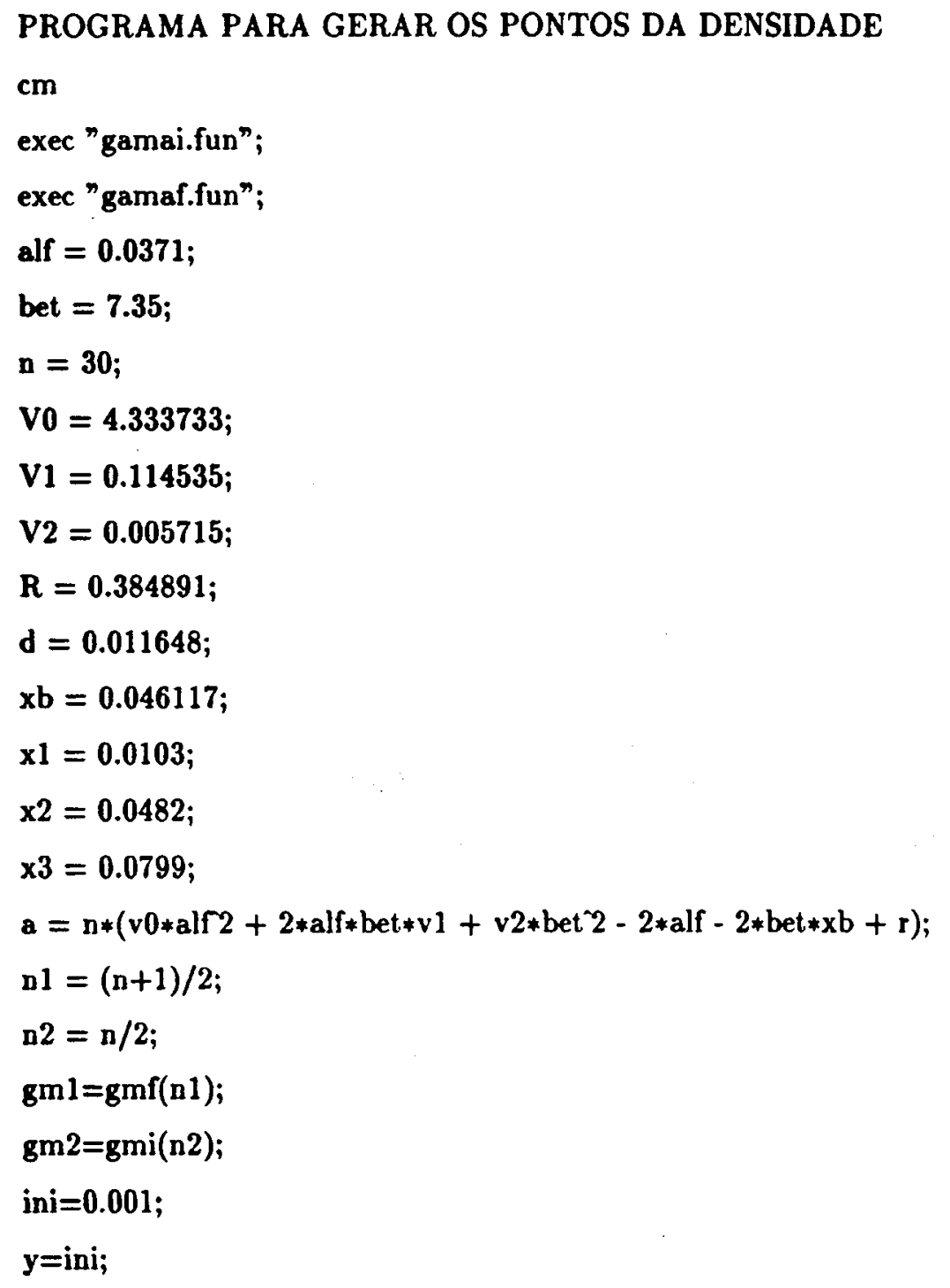




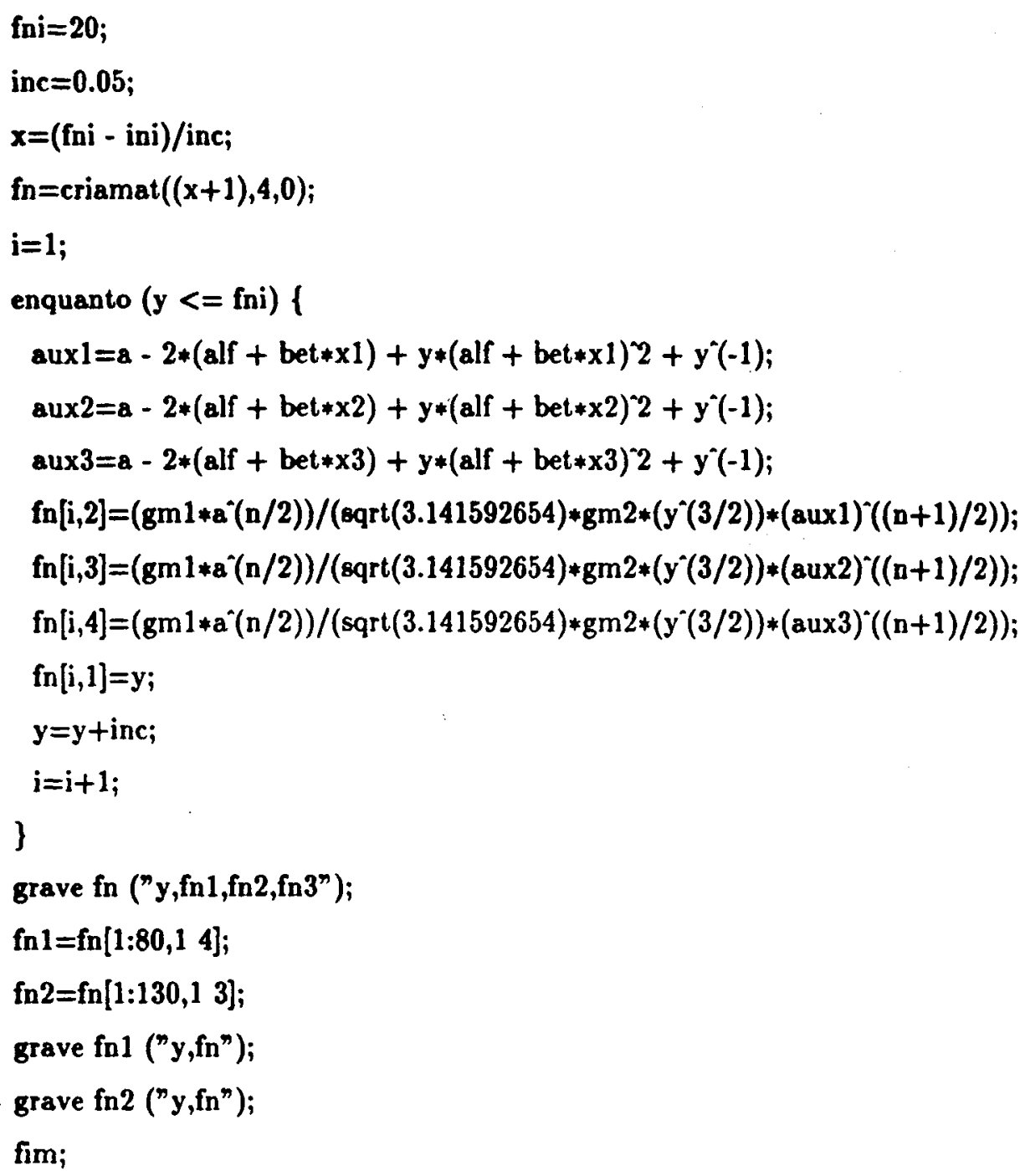

